Universitat Jaume I

Doctoral School

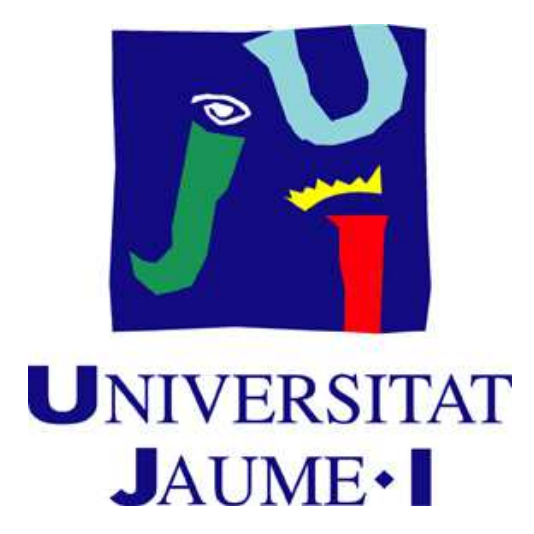

Global GeOmetry of Surfaces

DEFINED BY NON-POSITIVE AND

NEGATIVE AT INFINITY

VALUATIONS.

AUTHOR

Carlos Jesús Moreno Ávila

ADVISORS

Dr. Carlos Galindo Pastor

Dr. Francisco José Monserrat Delpalillo

Castelló de la Plana, April 2021 



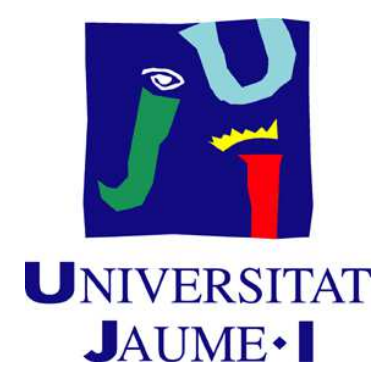

Programa de Doctorado en Ciencias

Escuela de Doctorado de la Universitat Jaume I

\section{GLOBAL GEOMETRY OF SURFACES DEFINED BY NON-POSITIVE AND NEGATIVE AT INFINITY VALUATIONS.}

Memoria presentada por Carlos Jesús Moreno Ávila para optar al grado de doctor por la Universitat Jaume I

DOCTORANDO

Carlos Jesús Moreno Ávila

\section{DIRECTORES}

Dr. Carlos Galindo Pastor

Dr. Francisco José Monserrat Delpalillo

$\begin{array}{ll}\text { MORENO } & \begin{array}{l}\text { Firmado } \\ \text { digitalmente por }\end{array} \\ \text { AVILA } & \text { MORENO AVILA } \\ \text { CARLOS } & \text { CARLOS JESUS - } \\ \text { JESUS - } & \text { 76027444R } \\ \text { 76027444R } & \text { Fecha: } 2021.04 .29 \\ 17: 19: 59+02 ' 00^{\prime}\end{array}$
GALINDO Signat digitalment PASTOR per GALINDO CARLOS - PASTOR CARLOS - 09271003W Data: 2021.04 .29 09271003W 21:18:30 +02'00'

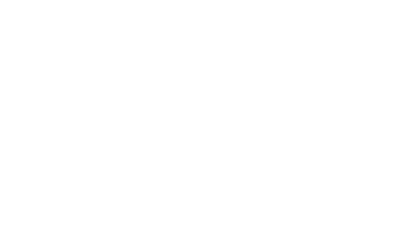

MONSERRAT Firmado DEL PALILLO MONSERRAT DEL FRANCISCO $\begin{array}{ll}18978577 N & \begin{array}{l}\text { Fecha: } 2021.04 .29 \\ 17: 59: 00+02^{\prime} 00^{\prime}\end{array}\end{array}$ 



\section{FINANCIACIÓN RECIBIDA}

- Ayuda predoctoral para la formación del personal investigador 2016 del Ministerio de Economía, Industria y Competitividad (MINECO):

BES-2016-076314

- Ayuda de la Universitat Jaume I destinada al estudiante de doctorado para la realización de actividades formativas en el año 2017.

- Participación en los proyectos financiados por MICINN/FEDER/AEI/UE:

MTM2015-65764-C3-2-P, MTM2016-81735-REDT, PGC2018-096446-B-C22 y RED2018-102583-T.

- Participación en los proyectos de la Universitat Jaume I:

P1-1B2015-02 y UJI-B2018-10. 



\section{Acknowledgments}

May the reader forgive me the discourtesy of following my wish to write the acknowledgments in my mother tongue.

A mis directores de tesis, Carlos Galindo y Francisco Monserrat, por su inestimable ayuda, por toda su paciencia y dedicación, por todas las conversaciones mantenidas y por sus consejos. Ha sido un verdadero placer trabajar con ellos. Sin lugar a dudas, sin ellos no hubiera llegado a buen puerto.

A los miembros del departamento de matemáticas de la Universitat Jaume I, por todo el afecto que he recibido durante todo este tiempo. Especialmente, a Fernando Hernando y Julio Moyano por su conversaciones e interrupciones en el despacho que me permitieron despejarme y desatascarme en mis cuentas. También a mis compañeros de doctorado de matemáticas y a los miembros del grupo Cantineros, por su amistad y apoyo durante todo este recorrido.

Mencionar a los miembros del departamento de matemáticas de la Universidad de Valladolid y del IMUVA por el recibimiento en la estancia de dos semanas que hice al principio de mi etapa doctoral y las posteriores visitas. Especialmente, nombrar a Antonio Campillo y Félix Delgado por invitarme y por las conversaciones mantenidas. También a los alumnos de doctorado, en particular, a Daniel Camazón por su apoyo y por sus rutas turísticas y de tapas por Valladolid.

I would like to thank the department of mathematics and the algebra section of KU Leuven for my stay of three months. In particular, I thank professor Wim Veys for the invitation, the hospitality and the work together. Also I would like to mention Marcel Rubió, Lena Vos and the remaining doctoral students of the algebra section for the wonderful time spending together.

Tampoco quiero olvidarme de mi periodo de formación en la Universidad de Extremadura. A todos los miembros del departamento de matemáticas y, particularmente, a María Ángeles Mulero e Ignacio Ojeda por iniciarme en cuarto curso de grado a la investigación en matemáticas, por su apoyo y por sus consejos. También mencionar a mi compañero de fatigas en grado y máster Daniel Morales, por todo el tiempo que hemos compartido juntos.

A lo largo de todo el camino que llevo recorrido he tenido la suerte de estar rodeado de una magnífica familia. A mis padres por su apoyo incondicional y por sus esfuerzos en ofrecerme lo mejor. A mi hermano, por todos los buenos ratos que 
hemos pasado juntos. A mis abuelos, a mis tíos, a mis primos y, especialmente, a la memoria de mi tía Alfonsa, cuya fuerza, coraje y lucha son y serán un ejemplo para mí.

Por último, y no por ello menos importante, a Alicia, mi compañera de aventuras, por todo su apoyo, su comprensión y su cariño incalculable mostrados cada día durante todo este tiempo a pesar de la desdicha de estar distanciados.

Esta tesis recoge el trabajo y el esfuerzo desempeñado durante cuatro años. En este tiempo he tenido el placer de disfrutar de la compañía de excelentes personas, tanto en el día a día, como en cada uno de los eventos que he participado. A todos ellos, muchas gracias.

"El buen paso, el regalo y el reposo allá se inventó para los blandos; mas el trabajo, la inquietud y las armas solo se inventaron e hicieron para aquellos que el mundo llama caballeros andantes, de los cuales yo, aunque indigno, soy el menor de todos." (Miguel de Cervantes, Quijote, I, XIII).

"La Matemática es una hermosa y arisca dama que solo muestra sus encantos a quienes porfían con ella." (Carlos Benítez, Lección inaugural del curso académico 2007/08 de la UEx). 


\section{Abstract}

We introduce the concepts of non-positive and negative at infinity plane valuation of a Hirzebruch surface and determine nice global and local geometric properties of the surfaces given by those divisorial valuations.

Let $\mathbb{F}_{\delta}$ be a Hirzebruch surface over an algebraically closed field, where $\delta$ is a non-negative integer. The concepts of non-positive and negative at infinity divisorial valuation were firstly introduced in [60] for valuations considered over the projective plane $\mathbb{P}^{2}$. We extend these concepts to divisorial valuations of $\mathbb{F}_{\delta}$, giving easy to check conditions to decide whether a valuation is of these types, and study positivity properties of the surfaces that they define. We prove that non-positive at infinity divisorial valuations of $\mathbb{F}_{\delta}$ are those divisorial valuations of $\mathbb{F}_{\delta}$ such that cone of curves of the surface that they define is minimally generated. Our results extend those in [60].

Non-positivity and negativity at infinity are also extended to the class of real valuations of $\mathbb{P}^{2}$ and $\mathbb{F}_{\delta}$. Their dual graphs are studied and compared according to the valuations they come from.

Finally, given a flag $E_{\bullet}=\{Z \supset E \supset\{q\}\}$, where $E$ is an exceptional divisor defining a non-positive at infinity divisorial valuation of $\mathbb{F}_{\delta}$ over $\mathbb{C}$ and $Z$ the rational surface given by $\nu_{E}$, we explicitly compute the Seshadri-type constant for pairs $\left(\nu_{E}, D\right)$, where $D$ is a big divisor on $\mathbb{F}_{\delta}$, and obtain the vertices of the NewtonOkounkov bodies of pairs $\left(E_{\bullet}, D\right)$. 



\section{Resumen}

Introducimos los conceptos de no positividad y negatividad en el infinito para valoraciones planas de una superficie de Hirzebruch y determinamos interesantes propiedades geométricas globales y locales de las superficies definidas por las valoraciones divisoriales que cumplen dicha condición.

Sea $\mathbb{F}_{\delta}$ una superficie de Hirzebruch sobre un cuerpo algebraicamente cerrado, donde $\delta$ es un entero no negativo. Los conceptos de valoración divisorial no positiva y negativa en el infinito fueron primeramente introducidos en [60] para valoraciones sobre $\mathbb{P}^{2}$. Nosotros extendemos estos conceptos a valoraciones divisoriales de $\mathbb{F}_{\delta}$, aportando una condición fácil de comprobar para decidir cuándo una valoración es de estos tipos, y estudiamos propiedades de positividad de las superficies que estas definen. En particular, probamos que las valoraciones divisoriales de $\mathbb{F}_{\delta}$ no positivas en el infinito son aquellas valoraciones divisoriales de $\mathbb{F}_{\delta}$ tales que el cono de curvas de la superficie que definen está generado por un número mínimo de generadores.

Los conceptos de no positividad y negatividad en el infinito también los extendemos para valoraciones reales de $\mathbb{P}^{2}$ y $\mathbb{F}_{\delta}$. Sus grafos duales son estudiados y comparados acorde a las valoraciones que les corresponden.

Sea una bandera $E_{\bullet}=\{Z \supset E \supset\{q\}\}$, donde $E$ es un divisor excepcional que define una valoración divisorial $\nu_{E}$ de $\mathbb{F}_{\delta}$ (sobre $\mathbb{C}$ ) no positiva en el infinito y $Z$ la superficie racional dada por $\nu_{E}$. En la última parte de la tesis calculamos explícitamente la constante de tipo Seshadri de pares $\left(\nu_{E}, D\right)$, donde $D$ es un divisor big en $\mathbb{F}_{\delta}$, y también obtenemos los vértices de los cuerpos de Newton-Okounkov de pares $\left(E_{\bullet}, D\right)$. 



\section{Contents}

Acknowledgments vii

Abstract

Resumen $\quad$ xi

List of Figures $\quad$ XV

Introduction 1

1 Preliminaries 13

1.1 Basic concepts . . . . . . . . . . . . . . . . . . . 13

1.2 Blowups and configurations . . . . . . . . . . . . . 17

1.3 Plane valuations . . . . . . . . . . . . . . . . . . . . 21

1.3.1 More invariants of plane valuations . . . . . . . . . . 27

1.4 Cones associated to a surface . . . . . . . . . . . . . . . . . 31

1.4.1 Convex cones . . . . . . . . . . . . . . . . . . . 31

1.4.2 Cone of curves of a surface . . . . . . . . . . . 35

1.5 Seshadri-type constants and $\mathrm{NO}$ bodies . . . . . . . . . . . . 40

1.5.1 Seshadri-type constants of divisorial valuations . . . . . . . . 40

1.5.2 NO bodies of big divisors on a surface . . . . . . . . . . . . 42

1.6 Rational surfaces . . . . . . . . . . . . . . . . . . . . 44

1.6.1 Hirzebruch surfaces . . . . . . . . . . . . . . . . 46

2 NPI and NI valuations of a rational surface 53

2.1 NPI valuations of the projective plane . . . . . . . . . . . . 54

2.2 Valuations of a Hirzebruch surface . . . . . . . . . . . . . . 55

2.3 The cone of curves of NPI special valuations . . . . . . . . . 57

2.4 The cone of curves of NPI non-special valuations . . . . . . . . . . 68

2.5 Discrete equivalence of NPI valuations . . . . . . . . . . . . . . . 79

2.5.1 An algorithm for obtaining the dual graphs of NPI valuations 83 
3 Seshadri-type constants and NO-bodies $\quad 87$

3.1 Seshadri-type constants for the projective plane . . . . . . . . . . 88

3.2 Seshadri-type constants for Hirzebruch surfaces . . . . . . . . . . . . 91

3.3 NO-bodies of NPI valuations . . . . . . . . . . . . . . . . . 94

3.3.1 NO-bodies of NPI special valuations . . . . . . . . . . 102

3.3 .2 NO-bodies of NPI non-special valuations . . . . . . . . . . 121

$\begin{array}{lrl}\text { Conclusions } & 133\end{array}$

$\begin{array}{lr}\text { Conclusiones } & 135\end{array}$

$\begin{array}{lr}\text { References } & 137\end{array}$ 


\section{List of Figures}

1.1 Dual graph of a divisorial valuation . . . . . . . . . . . . . . 24

1.2 Dual graph of an irrational valuation . . . . . . . . . . . . . . 24

1.3 Dual graph of a non-exceptional curve valuation . . . . . . . . . . 25

1.4 Dual graph of an exceptional curve valuation . . . . . . . . . . . . 25

1.5 Dual graph of infinitely singular valuation . . . . . . . . . . . . 26

1.6 Table of the types of plane valuations . . . . . . . . . . . 27

2.1 Dual graph of $\nu$ in Example 2.3.13 . . . . . . . . . . . . . . 67

2.2 Dual graph of $\nu$ in Example 2.4.13 . . . . . . . . . . . . . 78

2.3 Dual graphs in Example 2.5.7. . . . . . . . . . . . . . . . . . . . . . . . . . . .

2.4 Dual graphs in Example 2.5.8. . . . . . . . . . . . . . . . 86

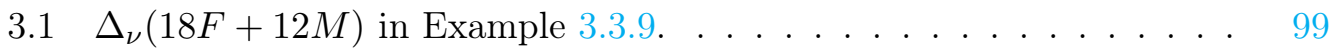

3.2 Local description of the cone of curves $\mathrm{NE}(Z)$ of a rational surface $Z$ given by a non-positive at infinity special divisorial valuation of $\mathbb{F}_{\delta} . \quad 108$

$3.3 \Delta_{\nu}(F+2 M)$ and $\mathfrak{C}(\nu) \cap \mathfrak{H}_{F+2 M}(\nu)$ in Example 3.3.22 . . . . . . 113

$3.4 \Delta_{\nu}(F+M)$ and $\mathfrak{C}(\nu) \cap \mathfrak{H}_{F+M}(\nu)$ in Example 3.3.25 . . . . . . 116

$3.5 \Delta_{\nu}(-2 F+M)$ and $\mathfrak{C}(\nu) \cap \mathfrak{H}_{-2 F+M}(\nu)$ in Example 3.3.28. . . . . 119

3.6 Local description of the cone of curves $\mathrm{NE}(Z)$ of a rational surface $Z$ given by a non-positive at infinity non-special divisorial valuation of $\mathbb{F}_{\delta} \cdot 126$

$3.7 \Delta_{\nu}(2 F+3 M)$ and $\mathfrak{C}(\nu) \cap \mathfrak{H}_{2 F+3 M}(\nu)$ in Example 3.3.46 . . . . . 131 



\section{Introduction}

Let $A_{1}(X)$ (respectively, $A^{1}(X)$ ) be the $\mathbb{R}$-vector space of numerical equivalence classes of one-cycles (respectively, numerical equivalence classes of Cartier divisors) of an $n$-dimensional complete algebraic scheme $X$ over an algebraically closed field $k$ of any characteristic. Assuming that $X$ is a smooth projective variety, $A^{1}(X)$ is a vector space of finite dimension. The dimension of both $A^{1}(X)$ and its dual $A_{1}(X)$ is called the Picard number of $X$ and written $\rho=\rho(X)$.

Given $X$ as above (projective and smooth), the cone of curves of $X, \mathrm{NE}(X)$, is the convex cone (see Subsection 1.4.1) of $A_{1}(X)$ spanned by the numerical equivalence classes of all effective 1-cycles of $X$ (positive combinations of integral curves); its closure $\overline{\mathrm{NE}}(X)$ is a closed convex cone. In addition, the numerically effective (nef) cone of $X, \operatorname{Nef}(X)$, is the convex cone in $A^{1}(X)$ generated by the numerical equivalence classes of Cartier divisors whose intersection product with all irreducible curves is non-negative (and it is also the dual cone of $\overline{\mathrm{NE}}(X)$ ). The previously introduced cones are relevant in the study of the geometry of the variety $X$. When $X$ is 2-dimensional, the case considered in this work, $A^{1}(X)$ is identified with $A_{1}(X)$ and the previously defined convex cones live in the same vector space of finite dimension. In addition, it holds that $\operatorname{Nef}(X) \subseteq \overline{\mathrm{NE}}(X)$. See [11, 94, 95, 21, 110] for some recent works with respect to $\overline{\mathrm{NE}}(X)$ and $[23,24,29,45]$ with respect to $\operatorname{Nef}(X)$.

Kleiman [76] also proved an important numerical criterion for ample divisors. It claims that the closure of the cone of curves $\overline{\mathrm{NE}}(X)$ except of the origin lies entirely in the positive halfspace of $A_{1}(X)$ determined by any ample Cartier divisor.

The cone $\overline{\mathrm{NE}}(X)$ plays a significant role in the minimal model program. This program aims a birational classification theory of higher-dimensional algebraic varieties and started at the end of the 20th century. The program pretends to extend (and include) the case of curves and the Enriques classification of algebraic surfaces. In the surfaces case, Enriques, Castelnuovo, Severi, Zariski, Mumford, Bombieri were important contributors. Some of the main mathematicians involved in the higher dimensional case are Mori, Kollar, Shokurov, Kawamata, Reid, Cascini and Birkar. Even when the program is in constant evolution, one can found an introduction in [77] and also in [88], where the Enriques classification of surfaces is included in the framework of the minimal model program. See [10] for a recent survey.

Let $K_{X}$ be a canonical divisor on a smooth projective complex variety $X$. Set 
$D$ any divisor on $X$ and write $\left.\overline{\mathrm{NE}}(X)\right|_{D_{\geq 0}}=\overline{\mathrm{NE}}(X) \cap D_{\geq 0}$ the subset of $\overline{\mathrm{NE}}(X)$ which is in the non-negative half-space given by $D$. The closure of the cone of curves has a particular structure which helps to construct minimal models for varieties. This fact was proved by Mori [93] for the 3-dimensional case, and later generalized by Kawamata [75] for arbitrary dimension. Indeed, assuming that the canonical divisor $K_{X}$ is not nef, the subset $\left.\overline{\mathrm{NE}}(X)\right|_{K_{X}<0}$ is generated by countable many rational curves and they can only accumulate on the hyperplane defined by the elements of $A_{1}(X)$ whose intersection product with $K_{X}$ vanishes. Even more, fixed an ample divisor $H$ and given a positive number $\varepsilon$, there are only finitely many curves $C_{1}, C_{2}, \ldots, C_{s}$ whose classes lie in the region $\left.\overline{\mathrm{NE}}(X)\right|_{K_{X}+\varepsilon H \leq 0}$ and then $\overline{\mathrm{NE}}(X)=$ $\left.\overline{\mathrm{NE}}(X)\right|_{K_{X}+\varepsilon H \geq 0}+\sum_{i=1}^{s} \mathbb{R}_{>0}\left[C_{i}\right]$. These curves $C_{i}$ generate extremal rays $R_{i}$ of $\overline{\mathrm{NE}}(X)$ and define maps which contract all the curves whose class lies in the extremal rays.

In general, even if $X$ is a smooth projective surface, there is no characterization of the fact that the cone $\overline{\mathrm{NE}}(X)$ is finitely generated (i.e. it is finite polyhedral and then $\overline{\mathrm{NE}}(X)=\mathrm{NE}(X)$ ). It is known (see [71, Chapter V, Problem 4.15] and [85, Section 1.5.D]) that if $Y$ is the surface obtained by blowing-up ten or more points of $\mathbb{P}^{2}$ in very general position, there are infinitely many smooth irreducible curves with self-intersection -1 (named (-1)-curves) and these curves span extremal rays of $\left.\overline{\mathrm{NE}}(Y)\right|_{K_{Y}<0}$. In addition, it is conjectured that the region $\left.\overline{\mathrm{NE}}(Y)\right|_{K_{Y}>0}$ is "almost circular" (i.e. is supported upon a spherical cone) [33, 34].

When $X$ is a smooth projective surface, the literature contains several works related to the structure of $\overline{\mathrm{NE}}(X)$. In [106] it is proved that the unique smooth surfaces whose cone of curves could have infinitely many -1-curves are the rational surfaces. In a complementary way, many authors, using different methods and under specific assumptions on rational surfaces $X$, have given either sufficient or equivalent conditions to the fact that $\overline{\mathrm{NE}}(X)$ is finite polyhedral (see for example [69, 89, 17, $55,70,56,60,35,50,51])$.

Valuations have been used in several areas of the mathematics to study different problems, including the classification of varieties. In 1882 Dedekind and Weber used valuations to treat Riemann surfaces, but the first axiomatic definition of valuation was given in 1912 by Kürschák. Valuations are important objects in the problem of resolution of singularities as one can see in the works of Zariski and Abhyankar [114, 115, 117, 1, 2]. Although Hironaka solved this problem in characteristic zero without using them, they seem to be a relevant tool for the case of positive characteristic [111]. Some recent works about valuations are [41, 112, 16, 96, 105, 97, 32, 6].

There is no classification of valuations. Nevertheless, valuations of the quotient field of regular two-dimensional local rings $(R, \mathfrak{m})$ centered at $\mathfrak{m}$ were classified by Zariski in terms of three invariants: the rank, the rational rank and the transcendence degree. These valuations are usually named plane valuations. A refinement of the previous classification was given by Spivakosky [109] in terms of dual graphs (see 
also [47] and [66]). Plane valuations have an interesting geometric point of view: they are in one-to-one correspondence with simple sequences of point blowups which start with the blowup of $\operatorname{Spec} R$ at $\mathfrak{m}$. That is, sequences of point blowups where each center (except the first one $\mathfrak{m}$ ) is a closed point in the last created exceptional divisor. These sequences need not to be finite and those which are correspond to divisorial valuations: those defined by the order at the last appearing exceptional divisor in the corresponding sequence of blowups.

Spivakovsky's classification divides plane valuations in five types, being divisorial valuations one of them. In this dissertation we will be particularly interested in two other types: irrational and exceptional curve valuations (see Section 1.3).

In the last years several authors have considered a class of plane valuations $\nu$ called non-positive at infinity. These valuations provide nice information about geometric global objects as the cone of curves of surfaces given by $\nu[20,60]$ or on parameters of good error-correcting codes linked to this class of valuations [57, 59]. Non-positive at infinity valuations form part of the so-called valuations centered at infinity used to study the dynamics of polynomial maps of the affine plane [47, 48, 49].

Recall that $k$ stands for an algebraically closed field of arbitrary characteristic and $\mathbb{P}^{2}:=\mathbb{P}_{k}^{2}$ the projective plane over $k$. Denote by $(X: Y: Z)$ projective coordinates in $\mathbb{P}^{2}$, by $L$ the projective line "at infinity" with equation $Z=0$, and by $p$ the point of $\mathbb{P}^{2}$ whose coordinates are $(1: 0: 0)$. Take affine coordinates $x=X / Z$ and $y=Y / Z$. Consider a plane valuation $\nu$ of the function field of $\mathbb{P}^{2}$ centered at $\mathcal{O}_{\mathbb{P}^{2}, p}$. The valuation $\nu$ is called non-positive at infinity if it holds that $\nu(f) \leq 0$ for all $f \in k[x, y] \backslash\{0\}$.

A sub-class of non-positive at infinity valuations was studied in [20]. Valuations in that sub-class are determined by those sequences of point blowups which remove the base points of pencils defined by the line at infinity $L$ and curves with only one place at infinity (i.e., a projective curve $C$ on $\mathbb{P}^{2}$ which is reduced and unibranched at $p$ and such that $C \cap L=\{p\})$. The authors show that the cone of curves of the surfaces defined by the previous valuations $\nu$ is generated by the smallest possible set of curves, that is the classes of the strict transform of $L$ and those of the strict transforms of the exceptional divisors created by the sequence of blowups given by $\nu$ (i.e. the cone of curves is regular). This result is extended in [60] proving that the divisorial valuations $\nu$ providing surfaces with regular cone of curves are exactly those which are non-positive at infinity. Even more, [60, Theorem 1] gives other two equivalent conditions to the result of the cone of curves: a numerical local property which is easy to check and the nefness of a certain divisor derived from $\nu$. [60] also presents sufficient and necessary conditions to the fact that $\nu(f)<0$ for all $f \in k[x, y] \backslash k$.

Non-positive at infinity valuations are also useful in coding theory. There is another sub-class of non-positive at infinity valuations which is very related to projective curves with only one place at infinity. Abhyankar and Moh [3, 4] studied 
these curves and proved, under certain conditions, a nice result called AbhyankarMoh (semigroup) Theorem, which determines the generators of the so-called semigroup at infinity. In [58], the authors show an analogue result to Abhyankar-Moh Theorem for the semigroup at infinity of plane valuations of the former sub-class $S_{\nu, \infty}=\{-\nu(f) \mid f \in k[x, y] \backslash\{0\}\}$ considering adequate fields $k$. This semigroup is the key to construct and determine parameters of a large set of error-correcting codes [57, 59].

Assume now for a while that $X$ is a smooth irreducible projective variety over $k=\mathbb{C}$ of dimension $n$.

Recall that a divisor $D$ on $X$ is big when $h^{0}\left(X, \mathcal{O}_{X}(m D)\right)$ grows like $m^{n}$ [85, Section 2.2]. The convex cone in $A^{1}(X)$ generated by the numerical equivalence classes of big divisors is called the big cone of $X$ and denoted $\operatorname{Big}(X)$. It holds that $\operatorname{Big}(X)=\operatorname{Int}(\overline{\mathrm{NE}}(X))$ [85, Theorem 2.2.26], where Int means topological interior, and, when $X$ is a surface, there is a locally finite decomposition of $\operatorname{Big}(X)$ into rational locally polyhedral subcones called Zariski chambers (see Subsection 1.5.2 and [7]).

Newton-Okounkov bodies of big divisors $D$ on varieties $X$ as above are convex sets used to study the asymptotic behaviour of linear systems $|m D|$, for $m \gg 0$. Firstly, they were introduced by Okounkov [99, 100] and afterwards developed independently by Lazarsfeld and Mustaţă [86], and Kaveh and Khovanskii [74]. Many authors are interested in these convex sets since they also reveal interesting information about invariants and positivity properties of divisors on $X$ [15, 104, 81, 79, 80, 82]. Other recent works about Newton-Okounkov bodies are [26, 27, 92, 12, 101].

Let us recall the definition of Newton-Okounkov body of a big divisor with respect to a flag of subvarieties of $X$. A flag of subvarieties $Y_{\bullet}$ of $X$ is a sequence of smooth irreducible subvarieties $Y_{i}$ of codimension $i$ in $X, 0 \leq i \leq n$ :

$$
Y_{\bullet}:=\left\{X=Y_{0} \supset Y_{1} \supset \ldots \supset Y_{n}=\{q\}\right\} .
$$

The flag $Y_{\bullet}$ defines a valuation $\nu_{Y_{\bullet}}$ of the function field $K(X)$ with rank $n$. Then the Newton-Okounkov body $\Delta_{\nu_{Y_{\bullet}}}(D)$ of a big divisor $D$ on $X$ with respect to $\nu_{Y_{\bullet}}$ (or $Y_{\bullet}$ ) is the closed convex hull of the set

$$
\bigcup_{m \geq 1}\left\{\frac{\nu_{Y \bullet}(f)}{m} \mid f \in H^{0}\left(X, \mathcal{O}_{X}(m D)\right) \backslash\{0\}\right\} .
$$

The Newton-Okounkov bodies $\Delta_{\nu_{\mathbf{\bullet}}}(D)$ are non-empty compact sets [86, 74, 13] and polygons when $X$ is a surface [82]. In higher dimensions they are extremely complicated and could be non-polyhedral even if we suppose that $X$ and $D$ satisfy good properties [82].

The bodies $\Delta_{\nu_{Y_{\bullet}}}(D)$ satisfy the following property:

$$
\operatorname{vol}_{X}(D)=n ! \operatorname{vol}_{\mathbb{R}^{n}}\left(\Delta_{\nu_{Y_{\bullet}}}(D)\right),
$$


where $\operatorname{vol}_{\mathbb{R}^{n}}$ means the standard volume and

$$
\operatorname{vol}_{X}(D):=\limsup _{m \rightarrow \infty} \frac{h^{0}\left(X, \mathcal{O}_{X}(m D)\right)}{m^{n} / n !}
$$

called the volume of $D$. This volume is a birational invariant of big divisors (see [85, Proposition 2.2.43 and Example 2.2.49]) which gives information about the asymptotic behaviour of their linear systems. We cite some works that study the volume of a divisor: [85, Section 2.2], [7] and [42]. The volume of an ample divisor $D$ coincides with its self-intersection number. Other recent works about the volume of big divisors are [14, 113, 98, 25].

Some authors have found a relation between the Newton-Okounkov bodies and Seshadri constants [79, 78], objects introduced by Demailly in order to study Fujita's conjecture [37]. One can see [39, 67, 87, 118, 68, 101] for recent works about Seshadri constants.

An explicit computation of the Newton-Okounkov bodies $\Delta_{\nu_{\mathbf{Q}}}(D)$ is a difficult task even if $X$ is a surface. In this last case, Lazarsfeld and Mustaţă [86] provide a generic way to describe these bodies. This description depends on the Zariski decomposition of certain divisors (see Subsection 1.5.2). More explicitly and roughly speaking, the authors describe in [86] the Newton-Okounkov bodies $\Delta_{\nu_{Y_{\mathbf{v}}}}(D)$ as convex sets whose vertices are determined by the Zariski decomposition of divisors $D_{t}=D-t C$, where $C$ is the smooth irreducible subvariety of codimension 1 in $Y_{\bullet}$ and $t$ is a (real) number such that $a \leq t \leq \mu(D, C)$, $a$ being the coefficient of $C$ in the negative part of the Zariski decomposition of $D$ and $\mu(D, C):=\sup \{s>0 \mid D-s C$ is big $\}$. In particular, the first components of the vertices of the bodies $\Delta_{\nu_{\mathbf{0}}}(D)$ are those values $t$ where the ray $\left[D_{t}\right]$ crosses into a different Zariski chamber [82]. Furthermore, [65] considers surfaces $X$ defined by divisorial valuations $\nu$ of the function field of $\mathbb{P}^{2}$ centered at some point $p \in \mathbb{P}^{2}$ and flags $E_{\bullet}=\{X \supset E \supset\{q\}\}$ where $E$ is the exceptional divisor defining $\nu\left(=\nu_{E}\right)$. In that paper, the authors show that the exceptional curve valuations centered at $p$ and valuations $\nu_{E_{\bullet}}$ defined by flags $E_{\bullet}$ are the same thing. Moreover, it is proved that the Newton-Okounkov body of the pullback of a general projective line $H$ with respect to $E_{\bullet}$ is a triangle or a quadrilateral, characterizing when one gets triangle or not. Even more, these Newton-Okounkov bodies are explicitly and completely computed when the valuation $\nu_{E}$ is non-positive at infinity. Previously, these Newton-Okounkov bodies were only characterized for surfaces defined by certain family of divisorial valuations [28].

On the other hand, some authors $[15,38,64]$ are interested in an invariant associated to the vanishing sequence of sections of a big line bundle $L, H^{0}(X, L)$, along a real valuation $\nu$ of the function field $K(X)$ centered at a local ring $(R, \mathfrak{m})$ (over an algebraically closed field $k$ ). This invariant is defined as

$$
\hat{\mu}_{L}(\nu):=\lim _{m \rightarrow \infty} a_{\max }(m L, \nu) / m
$$


where $a_{\max }(m L, \nu)$ is the last value of the vanishing sequence. This object provides similar information for valuations as the Seshadri constant does for points. We call $\hat{\mu}_{L}(\nu)$ the Seshadri-type constant for the pair $(L, \nu)$ (see [31] for a similar invariant). In general, computing the value $\hat{\mu}_{L}(\nu)$ is also a hard task, although there exists the bound $\hat{\mu}_{L}(\nu) \geq \sqrt{\operatorname{vol}(L) / \operatorname{vol}(\nu)}$, where $\operatorname{vol}(\nu)$ means volume of the valuation $\nu$, i.e.

$$
\operatorname{vol}(\nu)=\lim _{\alpha \rightarrow \infty} \frac{\operatorname{length}\left(R / \mathcal{P}_{\alpha}\right)}{\alpha^{n} / n !},
$$

$\mathcal{P}_{\alpha}$ being the set $\mathcal{P}_{\alpha}=\{f \in R \mid \nu(f) \geq \alpha\} \cup\{0\}$ (see [15]). Assuming $X=\mathbb{P}^{2}$, $L$ a projective line which does not go through $p$ and $\nu_{E}$ a divisorial valuation of the function field of $\mathbb{P}^{2}$ centered at $\mathcal{O}_{\mathbb{P}^{2}, p}$, the last bound is expressed as $\hat{\mu}_{L}\left(\nu_{E}\right) \geq$ $\sqrt{1 / \operatorname{vol}\left(\nu_{E}\right)}$ and, if the equality holds, the valuation $\nu_{E}$ is called minimal. This concept is strongly involved in a valuative conjecture formulated in [64] (see also [38]) which implies the well-known Nagata conjecture. In addition, $\hat{\mu}_{L}\left(\nu_{E}\right)$ can be geometrically understood as $\hat{\mu}_{L}\left(\nu_{E}\right)=\sup \left\{s>0 \mid L^{*}-s E\right.$ is big $\}$ (when $k$ has characteristic zero), where $L^{*}$ is the pull-back of $L$ on the surface defined by $\nu_{E}$, which establishes a relation with the Newton-Okounkov bodies [15, 28, 65]. When $\nu_{E}$ is non-positive at infinity, $\hat{\mu}_{L}\left(\nu_{E}\right)$ have been computed explicitly in [64].

Let $\mathbb{F}_{\delta}$ be the $\delta$ th Hirzebruch surface over an algebraically closed field $k, \delta$ being a non-negative integer. The projective plane $\mathbb{P}^{2}$ and the Hirzebruch surfaces $\mathbb{F}_{\delta}, \delta \neq 1$, constitute the classical minimal models for rational surfaces [9]. In this dissertation we mainly consider divisorial valuations $\nu$ of the function field of $\mathbb{F}_{\delta}$ centered at $\mathcal{O}_{\mathbb{F}_{\delta}, p}$, where $\mathbb{F}_{\delta}$ is any Hirzebruch surface and $p$ is a closed point of $\mathbb{F}_{\delta}$, which we name divisorial valuations of $\mathbb{F}_{\delta}$ for short. As algebraic and local objects, these valuations do not differ of those corresponding to quotient fields of bidimensional local rings; however our valuations will be considered over global objects giving rise to global information.

The main goals in this thesis are four:

1. To find adequate affine charts on $\mathbb{F}_{\delta}$ that allow us to establish a concept of non-positivity (or negativity) at infinity in a close way as it was done in the projective case. The main fact is that valuations $\nu$ which are non-positive (or negative) on non-zero regular functions on these charts give rise to surfaces $Z$ with interesting geometrical global properties.

2. To compare non-positive at infinity divisorial and real valuations of $\mathbb{P}^{2}$ with those of $\mathbb{F}_{\delta}$ and provide algorithms determining their corresponding dual graphs.

3. To extend the concept of minimality of divisorial valuations of $\mathbb{P}^{2}$ to divisorial valuations of $\mathbb{F}_{\delta}$ and computing the Seshadri-type constant $\hat{\mu}_{D}(\nu)$ for any nonpositive at infinity divisorial valuation of $\mathbb{F}_{\delta}$ and any big divisor $D$ on $\mathbb{F}_{\delta}$.

4. Finally, to explicitly determine the Newton-Okounkov bodies with respect to flags $E_{\bullet}=\{Z \supset E \supset\{q\}\}$ of divisors $D$ which are the pull-back of big 
divisors and $E$ is the last created exceptional divisor by the sequence of blowups corresponding to non-positive at infinity valuations $\nu$ of $\mathbb{F}_{\delta}$.

The main results of this thesis are stated and proved in the following papers accomplished jointly my advisors:

[63] C. Galindo, F. Monserrat and C.-J. Moreno-Ávila. Non-positive at infinity divisorial valuations of Hirzebruch surfaces. Rev. Mat. Complut., 33:349-372, 2020 .

[62] C. Galindo, F. Monserrat and C.-J. Moreno-Ávila. Seshadri-type constants and Newton-Okounkov bodies for non-positive at infinity valuations of Hirzebruch surfaces. Arxivmath:1905.03531, 2019.

[61] C. Galindo, F. Monserrat and C.-J. Moreno-Ávila. Discrete equivalence of non-positive at infinity plane valuations. Arxivmath:1911.06661, 2019.

We devote the remaining of this introduction to summarize the contents of this memoir.

Chapter 1 reviews concepts and results which we will use in the upcoming chapters mainly about Hirzebruch surfaces, divisors, blowups and plane valuations. Here we also establish several conventions and notation that we will follow in this work. We focus on the 2-dimensional case, but we provide several references which contain details in higher dimension.

In Chapter 2 we introduce non-positive and negative at infinity divisorial and irrational valuations of Hirzebruch surfaces $\mathbb{F}_{\delta}$. Valuations of $\mathbb{F}_{\delta}$ is a short name for valuations of the function field of $\mathbb{F}_{\delta}$ centered at $\mathcal{O}_{\mathbb{F}_{\delta}, p}$ for some $p \in \mathbb{F}_{\delta}$. We study global geometric properties associated to the surfaces defined by these valuations in the divisorial case. Finally, we compare the dual graphs which can correspond with non-positive at infinity divisorial and irrational valuations of $\mathbb{P}^{2}$ and $\mathbb{F}_{\delta}$ and provide an algorithm to obtain all dual graphs corresponding to the previous valuations. Here our ground fields are algebraically closed of arbitrary characteristic.

Being more specific, Section 2.1 recalls the concepts of non-positive and negative at infinity divisorial valuation of $\mathbb{P}^{2}$ and the main results that characterize these valuations.

In Section 2.2 we distinguish two types of divisorial valuations of $\mathbb{F}_{\delta}$, called special and non-special valuations (Definition 2.2.1). This distinction is due to the particular geometric structure of the Hirzebruch surfaces (Propositions 1.6.9 and 1.6.10) and the situation arising from considering a particular finite simple sequence of points blowups (Proposition 2.2.2). As a result, there exist two natural charts "at infinity". On the one hand, that one given by the points of $\mathbb{F}_{\delta}$ which belong to neither the fiber $F_{1}$ containing $p$ nor the special section $M_{0}$ on $\mathbb{F}_{\delta}$-the unique integral curve on $\mathbb{F}_{\delta}$ with non-positive self-intersection (Subsection 1.6.1). On the other hand, the chart 
given by the points which are neither in $F_{1}$ nor in a particular uniquely defined curve $M_{1} \neq M_{0}$.

In Section 2.3 we focus on the study of global and local geometric properties of the rational surfaces $Z$ defined by non-positive or negative at infinity special divisorial valuations $\nu_{n}$ of $\mathbb{F}_{\delta}$. We begin by considering certain divisors $\Lambda_{i}, 1 \leq i \leq n$, on $Z$ (Proposition 2.3.1) and prove that they satisfy several nice properties. We also describe the relation of these divisors on $\mathbb{F}_{1}$ with those used in [60] for treating a similar problem for valuations of $\mathbb{P}^{2}$; notice that $\mathbb{F}_{1}$ can be obtained by blowing-up a point in $\mathbb{P}^{2}$. Our main result is Theorem 2.3.7 which proves [60, Theorem 1] as a particular case. Next, we state that result.

Theorem A (Theorem 2.3.7). Let $\nu_{n}$ be a special divisorial valuation of the function field of $\mathbb{F}_{\delta}$ centered at $\mathcal{O}_{\mathbb{F}_{\delta}, p}, p$ being a closed point in $\mathbb{F}_{\delta}$. Set $Z$ the surface that $\nu_{n}$ defines. Denote by $\varphi_{F_{1}}$ (respectively, $\varphi_{M_{0}}$ ) the germ of the fiber $F_{1}$ (respectively, the special section $M_{0}$ ) at $p$. Consider the divisor $\Lambda_{n}$ mentioned previously and the last maximal contact value of $\nu_{n}, \bar{\beta}_{g+1}\left(\nu_{n}\right)$. Then the following conditions are equivalent:

(a) The valuation $\nu_{n}$ is non-positive at infinity.

(b) The divisor $\Lambda_{n}$ is nef.

(c) The inequality $2 \nu_{n}\left(\varphi_{M_{0}}\right) \nu_{n}\left(\varphi_{F_{1}}\right)+\delta \nu_{n}\left(\varphi_{F_{1}}\right)^{2} \geq \bar{\beta}_{g+1}\left(\nu_{n}\right)$ holds.

(d) The cone of curves $\operatorname{NE}(Z)$ is generated by the classes of the strict transforms on $Z$ of $F_{1}, M_{0}$ and the irreducible exceptional divisors associated with the composition of point blowups $\pi: Z \rightarrow \mathbb{F}_{\delta}$ given by $\nu_{n}$.

From our reasoning, we are able to prove that all the divisors $\Lambda_{i}, 1 \leq i \leq n$, are nef and effective when we suppose that $\nu_{n}$ is non-positive at infinity (Remark 2.3.9 and Corollary 2.3.11). Even more, each special divisorial valuation $\nu_{i}$, defined by the exceptional divisor $E_{i}$ created by the sequence of blowups $\pi$, is also non-positive at infinity (Corollary 2.3.10) and a non-positive at infinity special irrational valuation of $\mathbb{F}_{\delta}$ can be approached by suitable sequences of non-positive at infinity special divisorial valuations of $\mathbb{F}_{\delta}$ (Corollary 2.3.12).

Negative at infinity special divisorial valuations of $\mathbb{F}_{\delta}$ are also characterized in this memoir; next we state this result which corresponds to Theorem 2.3.14 and has algebraic and geometric connotations. It extends Theorem 2 of [60].

Theorem B (Theorem 2.3.14). Under the notations in Theorem A, the following conditions are equivalent:

(a) The valuation $\nu_{n}$ is negative at infinity.

(b) It holds that either $2 \nu_{n}\left(\varphi_{M_{0}}\right) \nu_{n}\left(\varphi_{F_{1}}\right)+\delta \nu_{n}\left(\varphi_{F_{1}}\right)^{2}=\bar{\beta}_{g+1}\left(\nu_{n}\right)$ and the Iitaka dimension of the divisor $\Lambda_{n}$ vanishes, or $2 \nu_{n}\left(\varphi_{M_{0}}\right) \nu_{n}\left(\varphi_{F_{1}}\right)+\delta \nu_{n}\left(\varphi_{F_{1}}\right)^{2}>$ $\bar{\beta}_{g+1}\left(\nu_{n}\right)$. 
(c) The inequality $\Lambda_{n} \cdot \tilde{C}>0$ holds for the strict transform on $Z, \tilde{C}$, of any curve $C$ on $\mathbb{F}_{\delta}, C \neq F_{1}, M_{0}$.

This dissertation also considers surfaces defined by non-special divisorial valuations $\nu_{n}$ of $\mathbb{F}_{\delta}$. We study algebraic and local and global geometric properties related to the above mentioned surfaces. In this case the family of divisors one needs to use is much bigger and more complex than in special case (Proposition 2.4.2). We show that it is enough to consider a set of divisors denoted $\Delta_{i}, \delta+1 \leq i \leq n$, (Lemma 2.4.7) to prove the main results of this section (Theorems 2.4.8 and 2.4.14) and their consequences (Remark 2.4.9 and Corollaries 2.4.10, 2.4.11 and 2.4.12).

Notice that our results on the cone of curves do not assume any positivity property for the canonical divisor of the considered rational surfaces.

Continuing with our summary of Chapter 2, Section 2.5 is devoted to compare dual graphs corresponding to the three types of non-positive at infinity divisorial and irrational valuations considered in this dissertation. As mentioned, valuations are essentially algebraic objects and then the valuations of Hirzebruch surfaces do not differ from valuations centered at points of other smooth surfaces. Nevertheless, it is interesting to attach them to the surface where they are centered and study the three types of non-positive at infinity valuations which have been defined because they also contain geometric information. Thus, we would like to know better the surfaces which they define and to try to find a more purely algebraic definition for them. As a suitable tuple of rational or real numbers is an equivalent datum to the dual graph of a valuation as ours (Section 1.3), we say that two real valuations are discretely equivalent if they have the same tuple (Definition 2.5.1). We provide numerical conditions involving the tuples corresponding to real valuations of $\mathbb{P}^{2}$ or $\mathbb{F}_{\delta}$ to decide which dual graphs admit valuations of some of the above types of non-positive at infinity valuations (Theorem 2.5.4). Furthermore, we define the sets of discrete classes corresponding to each type and show inclusions among them (Theorem 2.5.5 and Remark 2.5.6). Finally, we develop an algorithm computing the discrete equivalence classes that admit non-positive at infinity divisorial and irrational valuations (Subsection 2.5.1).

Chapter 3 studies Seshadri-type constants and Newton-Okounkov bodies of nonpositive at infinity valuations of the projective plane and Hirzebruch surfaces. Here we assume that $\mathbb{C}$ is our ground field.

We start by reviewing the concept of Seshadri-type constant $\hat{\mu}_{D}\left(\nu_{n}\right)$ for divisorial valuations $\nu_{n}$ of $\mathbb{P}^{2}$ or $\mathbb{F}_{\delta}$ and big divisors $D$ on the above surfaces. We also extend the concept of minimal valuation of $\mathbb{P}_{\mathbb{C}}^{2}:=\mathbb{P}^{2}$ (Definition 3.0.1); our extension works for valuations $\nu_{n}$ and divisors as above. Our definition for the value $\hat{\mu}_{D}\left(\nu_{n}\right)$ involves a limit, but it can be also interpreted geometrically as

$$
\hat{\mu}_{D}\left(\nu_{n}\right)=\sup \left\{s>0 \mid D^{*}-s E_{n} \text { is big }\right\}
$$


where $E_{n}$ is the exceptional divisor defining $\nu_{n}$ and $D^{*}$ is the pull-back of $D$ on the surface that $\nu_{n}$ defines. We will prove in Section 3.1 that our extended definition of minimal valuation coincides with that given in $[38,64]$ when applying to a divisorial valuation of $\mathbb{P}^{2}$. We also show in this section, when $\nu_{n}$ is a non-minimal valuation of $\mathbb{P}^{2}$, that the so-called supraminimal curve generates an extremal ray of $\overline{\mathrm{NE}}(Z), Z$ being the surface defined by $\nu_{n}$ (Corollary 3.1.6). This supraminimal curve is defined by the unique monic irreducible polynomial $f$ such that $\operatorname{deg}(f) \hat{\mu}_{L}\left(\nu_{n}\right)=\nu_{n}(f)([38$, Lemma 5.1] and [65, Lemma 3.10]).

Our Section 3.2 proves one of the main results of Chapter 3, which shows the value of $\hat{\mu}_{D}\left(\nu_{n}\right)$ for non-positive at infinity divisorial valuations of $\mathbb{F}_{\delta}$ and big divisors on $\mathbb{F}_{\delta}$ :

Theorem C (Theorem 3.2.1). Let $\nu_{n}$ be a non-positive at infinity divisorial valuation of the function field of $\mathbb{F}_{\delta}$ centered at $\mathcal{O}_{\mathbb{F}_{\delta}, p}, p \in \mathbb{F}_{\delta}$. Let $D$ be a big divisor that is linearly equivalent to $a F+b M$ on $\mathbb{F}_{\delta}$. Then

(a) If the valuation $\nu_{n}$ is special, then $\hat{\mu}_{D}\left(\nu_{n}\right)=(a+b \delta) \nu_{n}\left(\varphi_{F_{1}}\right)+b \nu_{n}\left(\varphi_{M_{0}}\right)$, where $\varphi_{F_{1}}$ (respectively, $\varphi_{M_{0}}$ ) is the germ of $F_{1}$ (respectively, $M_{0}$ ) at $p$.

(b) Otherwise, $\hat{\mu}_{D}\left(\nu_{n}\right)=a \nu_{n}\left(\varphi_{F_{1}}\right)+b \nu_{n}\left(\varphi_{M_{1}}\right)$, where $\varphi_{F_{1}}$ (respectively, $\left.\varphi_{M_{1}}\right)$ is the germ of $F_{1}$ (respectively, $M_{1}$ ) at $p$.

Theorem $\mathrm{C}$ allows us to characterize those non-positive at infinity divisorial valuation of $\mathbb{F}_{\delta}$ which are minimal with respect to a big and nef divisor on $\mathbb{F}_{\delta}$ (Corollary 3.2.3).

Section 3.3 is devoted to study the Newton-Okounkov bodies of the pull-back on surfaces $Z$ of big divisors $D$ on either the projective plane $\mathbb{P}^{2}$ or Hirzebruch surfaces $\mathbb{F}_{\delta}$ with respect to flags $E_{\bullet}=\left\{Z \supset E_{r} \supset\left\{p_{r+1}\right\}\right\}$, where $E_{r}$ are exceptional divisors defining divisorial valuations $\nu_{r}$ of $\mathbb{P}^{2}$ or $\mathbb{F}_{\delta}$ and $Z$ the surface given by $\nu_{r}$. For short, we will denote these Newton-Okounkov bodies by $\Delta_{\nu}(D)$ (see Definition 3.3.3). Notice that following [65, Section 3.2], it can be proved that exceptional curve valuations $\nu$ of $\mathbb{P}^{2}$ or $\mathbb{F}_{\delta}$ correspond to valuations $\nu_{E_{\bullet}}$ attached to the flags $E_{\bullet}$ as above. These exceptional curve valuations $\nu$ have two components $\nu=\left(v_{1}, v_{2}\right)$, where $v_{1}=\nu_{r}$ is the divisorial valuation defined by $E_{r}$. We introduce concepts like special, non-special, non-positive at infinity and minimal exceptional curve valuation of a Hirzebruch surface which depend on the first component of the exceptional curve valuation (Definitions 3.3.1 and 3.3.2). Key objects in this section are the so-called maximal contact values of some divisorial valuations $\nu_{i}$ defined by the exceptional divisors $E_{i}$ appearing in the sequence of point blowups corresponding to $\nu_{r}$. These values are denoted $\left\{\bar{\beta}_{j}\left(\nu_{i}\right)\right\}_{j=0}^{g+1}$ and we are only interested in the indices $i=r$ and $i=\eta$ (Subsection 1.3.1). We set $g^{*}+2$ the number of maximal contact values of the exceptional curve valuation $\nu$. Lemma 3.3.6 considers maximal contact values and plays an important role in our explicit description of Newton-Okounkov bodies. 
The first main result in Section 3.3 describes the Newton-Okounkov bodies $\Delta_{\nu}(D)$ of big divisors on $\mathbb{P}^{2}$ and $\mathbb{F}_{\delta}$ of minimal exceptional curve valuations $\nu$.

Theorem D (Theorem 3.3.7). Let $E_{\bullet}=\left\{Z \supset E_{r} \supset\left\{p_{r+1}\right\}\right\}$ be a flag and $\nu=\nu_{\bullet}$ its attached exceptional curve valuation. Set $D$ a big divisor on either $\mathbb{P}^{2}$ or $\mathbb{F}_{\delta}$. Then, the valuation $\nu$ is minimal if and only if the Newton-Okounkov body $\Delta_{\nu}(D)$ is a triangle whose vertices are $(0,0),\left(\hat{\mu}_{D}\left(\nu_{r}\right), \frac{\hat{\mu}_{D}\left(\nu_{r}\right) \bar{\beta}_{0}\left(\nu_{\eta}\right)}{\bar{\beta}_{0}\left(\nu_{r}\right)}\right)$ and $\left(\hat{\mu}_{D}\left(\nu_{r}\right), \frac{\hat{\mu}_{D}\left(\nu_{r}\right) \bar{\beta}_{g^{*}}\left(\nu_{\eta}\right)}{\bar{\beta}_{g^{*}}\left(\nu_{r}\right)}\right)$, when $p_{r+1} \in E_{r} \cap E_{\eta}$ with $\eta \neq r$. If $p_{r+1}$ is a free point, the triangle is given by the vertices $(0,0),\left(\hat{\mu}_{D}\left(\nu_{r}\right), 0\right)$ and $\left(\hat{\mu}_{D}\left(\nu_{r}\right), \frac{\hat{\mu}_{D}\left(\nu_{r}\right)}{\bar{\beta}_{g^{*}+1}\left(\nu_{r}\right)}\right)$.

An explicit description of the Newton-Okounkov bodies of flags as above for nonminimal valuations $\nu$ is an open problem. Section 3.3 solves it when the valuations $\nu$ are non-positive at infinity. Notice that [65] solves the case where $\nu$ is a non-minimal exceptional curve valuations of $\mathbb{P}^{2}$.

We divide our study in two subsections (Subsections 3.3.1 and 3.3.2) providing a description that depends on the fact that the exceptional curve valuation is either special or non-special.

Following ideas in [86] and [82], we start by looking for suitable values $t_{i}$ which will be the first components of the vertices of the Newton-Okounkov bodies to be described (Lemmas 3.3.13 and 3.3.34). Then, we obtain the corresponding Zariski decompositions (Propositions 3.3.17 and 3.3.38). Recall that these values $t_{i}$ are those where the ray $[D]-t\left[E_{r}\right]$ crosses into a different Zariski chamber and therefore they characterize locally the structure of the interior of the cone of curves $\mathrm{NE}(Z)$ according to the exceptional curve valuation $\nu$ is either minimal or non-minimal (Remarks $3.3 .15,3.3 .18,3.3 .36$ and 3.3 .39$)$. The ray $\left[D^{*}\right]-t\left[E_{r}\right]$ crosses a unique Zariski chamber when $\nu$ is minimal with respect to $D$, and the same holds in a particular case when $\nu$ is special and non-minimal with respect to $D$. The ray $\left[D^{*}\right]-t\left[E_{r}\right]$ crosses at least two Zariski chamber when $\nu$ is non-special and non-minimal with respect to $D$.

We conclude this introduction by explaining that the main results in these subsections are explicit descriptions of the Newton-Okounkov bodies $\Delta_{\nu}(D)$ of big divisors with respect to a non-minimal non-positive at infinity exceptional curve valuation $\nu$ of $\mathbb{F}_{\delta}$ (see Theorems 3.3.21, 3.3.24, 3.3.27, 3.3.42 and 3.3.45). As a consequence of these explicit calculations, we show that the vertices of our Newton-Okounkov bodies depend only on the expression of $D$, the volume of $\nu$ and the values of the germs at $p$ of the fibre and sections on $\mathbb{F}_{\delta}$ whose strict transforms (together with those of the exceptional divisors) span the cone of curves. As a particular case of the results of the special exceptional curve valuations of $\mathbb{F}_{\delta}$, we obtain the Newton-Okounkov body computed in [65, Corollary 5.2] with respect to a non-positive at infinity exceptional curve valuation of $\mathbb{P}^{2}$ (Corollary 3.3.29). 



\section{Chapter 1}

\section{Preliminaries}

In this chapter we introduce the concepts, notation and conventions needed to develop this dissertation. We restrict the considered objects to the 2-dimensional case since we will always work there, although almost all of them can be extended to higher dimension. The style of this first part is descriptive due to its compilatory nature. Unless otherwise stated, our ground fields are algebraically closed of arbitrary characteristic.

\section{$1.1 \quad$ Basic concepts}

We start by collecting some concepts of algebraic geometry. We mainly follow [71]. Other useful references for us are [102, 107] and [108].

Let $k$ be an algebraically closed field of arbitrary characteristic and $k^{*}:=k \backslash\{0\}$. A variety is an integral separated scheme of finite type over $k$. Varieties of dimension 2 are named surfaces.

Set $Z$ a surface and $p$ a closed point of $Z$ (i.e., an irreducible 0-dimensional closed subscheme on $Z$ ). We denote by $\mathcal{O}_{Z}$ the structural sheaf of $Z$, by $\mathcal{O}_{Z, p}$ the stalk of $\mathcal{O}_{Z}$ at $p$ and by $\mathfrak{m}_{p}$ the maximal ideal of the local ring $\mathcal{O}_{Z, p}$. The elements of the stalk $\mathcal{O}_{Z, p}$ are called germs. It is said that $p$ is a smooth point of $Z$ if the local ring $\mathcal{O}_{Z, p}$ is regular. Otherwise, $p$ is a singular point (or a singularity) of $Z$. Moreover, the surface $Z$ is called to be smooth, non-singular or regular if every point of $Z$ is smooth. In this work, from now on, surface means 2-dimensional smooth projective variety and will be usually denoted by $Z$.

Let $G$ be a prime divisor (i.e., a closed integral subscheme of codimension 1) on $Z$ and $\eta$ its generic point. Then, the local ring $\mathcal{O}_{Z, \eta}$ is a discrete valuation ring with quotient field $K(Z)$, the function field of $Z$. If $\nu_{G}$ denotes the corresponding discrete valuation (see Section 1.3) then, for all non-zero element $f$ of $K(Z)$, the order of $f$ along $G$, denoted $\operatorname{ord}_{G}(f)$, is defined as $\nu_{G}(f)$.

A Weil divisor $D$ on $Z$ is an element of the free $\mathbb{Z}$-module $\operatorname{Div}(Z)$ generated by 
the prime divisors on $Z$. Thus, $D$ can be written

$$
D=n_{1} G_{1}+n_{2} G_{2}+\cdots+n_{r} G_{r}
$$

where $G_{i}$ are prime divisors on $Z$ and $n_{i}$ integer numbers. If $n_{i}=0$ for all $i$, we write $D=0$; if $n_{i} \geq 0$ for all $i$ and some of them is strictly positive, then $D$ is called to be effective or a curve. The union $\cup_{n_{i} \neq 0} G_{i}$ is called the support of $D$ and it is denoted by $\operatorname{supp}(D)$. If $C$ is a curve on $Z$ passing through $p, \varphi_{C, p}$ represents the germ of the curve $C$ at the point $p$. Often, we only use $\varphi_{C}$ if no confusion arises.

Let $f$ be a non-zero rational function of $K(Z)$. The Weil divisor of $f$, denoted by $\operatorname{div}(f)$, is defined as

$$
\operatorname{div}(f):=\sum_{G} \operatorname{ord}_{G}(f) G
$$

where the sum runs over the prime divisors $G$ on $Z$. A Weil divisor which equals the divisor of a rational function is named principal divisor. We say that two Weil divisors $D$ and $D^{\prime}$ are linearly equivalent, written $D \sim D^{\prime}$, if $D-D^{\prime}$ is a principal divisor. This is an equivalence relation on $\operatorname{Div}(Z)$ and we denote by $\operatorname{Cl}(Z)$ the quotient group $\operatorname{Div}(Z) / \sim$.

Let $\mathcal{K}$ be the constant sheaf corresponding to the function field $K(Z)$ [71, Chapter II, Example 1.0.3]. Denote by $\mathcal{K}^{*}$ (respectively, $\mathcal{O}_{Z}^{*}$ ) the sheaf of invertible elements in the sheaf of rings $\mathcal{K}$ (respectively, $\mathcal{O}_{Z}$ ). A Cartier divisor $D$ on $Z$ is a global section of the sheaf $\mathcal{K}^{*} / \mathcal{O}_{Z}^{*}$. As a result, a Cartier divisor can be described by giving a family of pairs $\left\{\left(U_{i}, f_{i}\right)\right\}_{i \in I}$, where $\left\{U_{i}\right\}_{i \in I}$ is an open covering of $Z$ and $f_{i} \in \Gamma\left(U_{i}, \mathcal{K}^{*}\right)$ is such that $f_{i} / f_{j} \in \Gamma\left(U_{i} \cap U_{j}, \mathcal{O}_{Z}^{*}\right)$ for all $i, j \in I$. A Cartier divisor $\left\{\left(U_{i}, f_{i}\right)\right\}_{i \in I}$ is principal if it is in the image of the natural map $\Gamma(Z, \mathcal{K}) \rightarrow \Gamma\left(Z, \mathcal{K}^{*} / \mathcal{O}_{Z}^{*}\right)$, i.e. all the functions $f_{i}$ are restrictions of the same rational function $f \in K(Z)$. We say that a Cartier divisor $\left\{\left(U_{i}, f_{i}\right)\right\}_{i \in I}$ is effective when $f_{i} \in \Gamma\left(U_{i}, \mathcal{O}_{U_{i}}\right)$ for all $i \in I$.

Set $D_{1}$ and $D_{2}$ two Cartier divisors defined by $\left\{\left(U_{i}, f_{i}\right)\right\}_{i \in I}$ and $\left\{\left(V_{j}, g_{j}\right)\right\}_{j \in J}$, respectively. We define the sum $D_{1}+D_{2}$ as the Cartier divisor given by the set $\left\{\left(U_{i} \cap V_{j}, f_{i} g_{j}\right)\right\}_{i \in I, j \in J}$. With this operation the set of Cartier divisors is an abelian group. We say that $D_{1}$ and $D_{2}$ are linearly equivalent, $D_{1} \sim D_{2}$, if their difference is a principal divisor. This is an equivalence relation on the set of Cartier divisors and we denote by $\mathrm{CaCl}(Z)$ its quotient group.

An invertible sheaf $\mathcal{L}$ on $Z$ is a locally free $\mathcal{O}_{Z}$-module of rank 1 . Set $\mathcal{L}_{1}$ and $\mathcal{L}_{2}$ two invertible sheaves on $Z$, then the tensor product $\mathcal{L}_{1} \otimes \mathcal{O}_{Z} \mathcal{L}_{2}\left(\mathcal{L}_{1} \otimes \mathcal{L}_{2}\right.$ for short) is also an invertible sheaf. In addition, if $\mathcal{L}$ is an invertible sheaf on $Z$, there exists an invertible sheaf $\mathcal{L}^{-1}$ on $Z$ such that $\mathcal{L} \otimes \mathcal{L}^{-1} \cong \mathcal{O}_{Z}$ ([71, Chapter II, Proposition 6.12]). The group of isomorphism classes of invertible sheaves on $Z$ under the tensor product is called Picard group of $Z$ and denoted $\operatorname{Pic}(Z)$.

Set $D$ a Cartier divisor on a surface $Z$ defined by $\left\{\left(U_{i}, f_{i}\right)\right\}_{i \in I}$. The sheaf associated to $D$, denoted $\mathcal{O}_{Z}(D)$, is the sub-sheaf of $\mathcal{K}$ defined by taking $\mathcal{O}_{Z}(D)$ to be 
the sub- $\mathcal{O}_{Z}$-module of $\mathcal{K}$ generated by $f_{i}^{-1}$ on $U_{i}$. This is well-defined because $f_{i} / f_{j}$ is invertible in $U_{i} \cap U_{j}$, so $f_{i}^{-1}$ and $f_{j}^{-1}$ generate the same $\mathcal{O}_{Z}$-module.

Proposition 1.1.1. [71, Chapter II, Proposition 6.13] Let $Z$ be a surface and $\mathcal{O}_{Z}$ its structural sheaf. Then:

(a) for any Cartier divisor $D, \mathcal{O}_{Z}(D)$ is an invertible sheaf. The map $D \mapsto \mathcal{O}_{Z}(D)$ gives a 1-1 correspondence between Cartier divisors on $Z$ and invertible subsheaves of $\mathcal{K}$.

(b) given two Cartier divisors, $D_{1}$ and $D_{2}$, the sheaf associated to $D_{1}-D_{2}$ is isomorphic to $\mathcal{O}_{Z}\left(D_{1}\right) \otimes \mathcal{O}_{Z}\left(D_{2}\right)^{-1}$.

(c) two Cartier divisors, $D_{1}$ and $D_{2}$, are linearly equivalent if and only if its associated invertible sheaves $\mathcal{O}_{Z}\left(D_{1}\right)$ and $\mathcal{O}_{Z}\left(D_{2}\right)$ are isomorphic.

All the surfaces $Z$ we are going to consider are noetherian, integral, separated locally factorial schemes. So, by [71, Chapter II, Proposition 6.11], the group of the Weil divisors, $\operatorname{Div}(Z)$, is isomorphic to the group of Cartier divisors and principal divisors correspond under this isomorphism. Therefore, we can say that a Cartier divisor is principal (respectively, effective) if it corresponds to a principal (respectively, effective) Weil divisor ([71, Chapter II, Remark 6.17.1]). As a consequence, in the future, we will use the word divisor, without specifying if it is a Weil or Cartier divisor, and also a curve on $Z$ and its corresponding effective divisor will be denoted by the same letter. In addition, $\operatorname{Pic}(Z)$ is isomorphic to the groups $\mathrm{Cl}(Z)$ and $\mathrm{CaCl}(Z)$ ([71, Chapter II, Proposition 6.15 and Corollary 6.16]). From now we will use this isomorphism and we will identify $\operatorname{Pic}(Z)$ with the group of the divisors on $Z$ modulo linear equivalence. The element of $\operatorname{Pic}(Z)$ defined by a divisor $D$ will be denoted $[D]$.

Intersection multiplicity and intersection number on surfaces are important tools in this work. Let us recall them.

Let $Z$ be a surface as above and suppose that $C_{1}$ and $C_{2}$ are two curves on $Z$ that meet at a point $p \in Z$. The intersection multiplicity $\left(\varphi_{C_{1}}, \varphi_{C_{2}}\right)_{p}$ of $C_{1}$ and $C_{2}$ at $p$ is defined to be

$$
\left(\varphi_{C_{1}}, \varphi_{C_{2}}\right)_{p}:=\operatorname{dim}_{k} \mathcal{O}_{Z, p} /\left\langle\varphi_{C_{1}}, \varphi_{C_{2}}\right\rangle .
$$

Moreover, it holds that $\left(\varphi_{C_{1}}, \varphi_{C_{2}}\right)_{p}=1$ if and only if $\varphi_{C_{1}}$ and $\varphi_{C_{2}}$ generate the maximal ideal $\mathfrak{m}_{p}$ of $\mathcal{O}_{Z, p}$. In this case, it is said that $C_{1}$ and $C_{2}$ are transversal at $p$, or meet transversally at $p$ ([9, Chapter I, Definition I.3]). We will understand that $\left(\varphi_{C_{1}}, \varphi_{C_{2}}\right)_{p}=0$ when $C_{1}$ or $C_{2}$ does not pass through $p$.

Theorem 1.1.2. [71, Chapter V, Theorem 1.1] Keep the previous notation. There is a unique pairing $\operatorname{Div}(Z) \times \operatorname{Div}(Z) \rightarrow \mathbb{Z}$, denoted by $D_{1} \cdot D_{2}$ for any two divisors $D_{1}, D_{2}$, such that 
(a) if $D_{1}$ and $D_{2}$ are non-singular curves meeting transversally, then $D_{1} \cdot D_{2}=$ \# $\left(D_{1} \cap D_{2}\right)$, the number of points of $D_{1} \cap D_{2}$,

(b) it is symmetric: $D_{1} \cdot D_{2}=D_{2} \cdot D_{1}$,

(c) it is additive: $\left(D_{1}+D_{2}\right) \cdot D_{3}=D_{1} \cdot D_{3}+D_{2} \cdot D_{3}$, for all $D_{3} \in \operatorname{Div}(Z)$,

(d) it depends only on the linear equivalence classes: if $D_{1}$ and $D_{2}$ are two linearly equivalent divisors then $D_{1} \cdot D_{3}=D_{2} \cdot D_{3}$, for all $D_{3} \in \operatorname{Div}(Z)$.

$D_{1} \cdot D_{2}$ is usually called the intersection number of $D_{1}$ and $D_{2}$.

The following proposition connects intersection number and intersection multiplicity.

Proposition 1.1.3. [71, Chapter V, Proposition 1.4] If $C_{1}$ and $C_{2}$ are curves on a surface $Z$ having no common irreducible component then

$$
C_{1} \cdot C_{2}=\sum_{p \in C_{1} \cap C_{2}}\left(\varphi_{C_{1}}, \varphi_{C_{2}}\right)_{p}
$$

To compute the self-intersection of a divisor $D$ on $Z$, that is, the intersection number $D^{2}:=D \cdot D$, linear equivalence must be used (see [71, Chapter V, Example 1.4.1]). In addition, using this concept we can define a numerical invariant on $Z$. Let $\Omega_{Z / k}$ be the sheaf of differentials of $Z$ over $k$ and $\omega_{Z}=\wedge^{2} \Omega_{Z / k}$ the canonical sheaf. A canonical divisor on $Z$ is any divisor whose associated invertible sheaf is $\omega_{Z} \cdot K_{Z}$ will denote an arbitrary canonical divisor of $Z$ and its self-intersection depends only on the surface $Z$.

The intersection number of two classes $\left[D_{1}\right]$ and $\left[D_{2}\right]$ of $\operatorname{Pic}(Z)$ is defined as intersection number $D_{1} \cdot D_{2}$ of any two representatives $D_{1}$ of $\left[D_{1}\right]$ and $D_{2}$ of $\left[D_{2}\right]$. Moreover, we say that $D_{1}$ (respectively, $\left[D_{1}\right]$ ) is orthogonal to $D_{2}$ (respectively, $\left[D_{2}\right]$ ) whenever $D_{1} \cdot D_{2}=0$ (respectively, $\left[D_{1}\right] \cdot\left[D_{2}\right]=0$ ). We denote by $D^{\perp}$ (respectively, $[D]^{\perp}$ ) the set of divisors (respectively, the classes of divisors) on $Z$ which are orthogonal to $D$ (respectively, $[D]$ ).

To conclude this section, we introduce several more concepts.

Set $\mathcal{L}$ an invertible sheaf on $Z$. By [71, Chapter II, Theorem 5.19], $H^{0}(Z, \mathcal{L})$ is a finite-dimensional $k$-vector space. Fixed $s \in H^{0}(Z, \mathcal{L})$, we will denote by $\operatorname{div}(s)_{0}$ the divisor of zeros of $s$, that is, the divisor defined by $\left\{U_{i}, \phi_{U_{i}}\left(\left.s\right|_{U_{i}}\right)\right\}_{i \in \mathcal{I}}$, where $\left\{U_{i}\right\}_{i \in \mathcal{I}}$ is an open covering of $Z$ and $\phi_{U_{i}}: \mathcal{L}\left(U_{i}\right) \rightarrow \mathcal{O}_{Z}\left(U_{i}\right)$ the isomorphism given by $\mathcal{L}$.

Proposition 1.1.4. [71, Chapter II, Proposition 7.7] Let $Z$ be a surface and $D$ a divisor on $Z$. Set $\mathcal{L}=\mathcal{O}_{Z}(D)$ the corresponding invertible sheaf. Then

(a) For each non-zero $s \in H^{0}(Z, \mathcal{L})$, the divisor of zeros of $s$ is an effective divisor linearly equivalent to $D$.

(b) Every effective divisor linearly equivalent to $D$ is $\operatorname{div}(s)_{0}$ for some $s \in H^{0}(Z, \mathcal{L})$. 
(c) Two sections $s, s^{\prime} \in H^{0}(Z, \mathcal{L})$ have the same divisor of zeros if and only if there is a $\lambda \in k^{*}$ such that $s^{\prime}=\lambda s$.

A complete linear system on $Z$ is the set (which might be empty) of effective divisors linearly equivalent to some given divisor $D$. We denote it by $|D|$. When $\mathcal{L}$ is isomorphic to the invertible sheaf $\mathcal{O}_{Z}(D)$ associated to $D$, by the above result, one has a one-to-one correspondence $\left(H^{0}(Z, \mathcal{L}) \backslash\{0\}\right) / k^{*} \rightarrow|D|$ defined by $s \mapsto \operatorname{div}(s)_{0}$, which gives $|D|$ a structure of projective space over $k$.

Proposition 1.1.5. [77, Lemma 1.20] If $D$ is a divisor on a surface $Z$ such that $D^{2}>0$, then either $|n D| \neq \emptyset$ or $|-n D| \neq \emptyset$ for $n$ large enough.

Any $k$-vector subspace $\mathfrak{d}$ of a complete linear system $|D|$ is called linear system. In the case of $\mathcal{L}$ being isomorphic to $\mathcal{O}_{Z}(D)$, the linear system $\mathfrak{d}$ corresponds to a $k$-vector subspace $W \subseteq H^{0}(Z, \mathcal{L})$, where $W=\left\{s \in H^{0}(Z, \mathcal{L}) \mid \operatorname{div}(s)_{0} \in \mathfrak{d}\right\} \cup\{0\}$, and the dimension of $\mathfrak{d}$, denoted $\operatorname{dim}(\mathfrak{d})$, is defined as the dimension of $\mathfrak{d}$ as projective space, that is, $\operatorname{dim}(\mathfrak{d})=\operatorname{dim}(W)-1$. A point $p \in Z$ is said to be a base point of a linear system $\mathfrak{d}$ if $p \in \operatorname{supp}(D)$ for all $D \in \mathfrak{d}$. A linear system $\mathfrak{d}$ is base-point-free when $\mathfrak{d}$ does not have base points.

Proposition 1.1.6. [71, Chapter II, Lemma 7.8] Let $\mathfrak{d}$ be a linear system on $Z$ corresponding to a subspace $W \subseteq H^{0}(Z, \mathcal{L})$. Then, $\mathfrak{d}$ is base-point-free if and only if $\mathcal{L}$ is generated by its global sections in $W$.

To finish, the Iitaka dimension [72] of a divisor $D$ on $Z$ is defined as $\kappa(D):=-\infty$ if $|n D|=\emptyset$ for all $n \in \mathbb{Z}_{>0}$ and, otherwise,

$$
\kappa(D):=\kappa(Z, D)=\max \left\{\operatorname{dim} \phi_{|n D|}(Z)\right\},
$$

where $n$ runs over $\left\{m \in \mathbb{Z}_{>0} \mid H^{0}\left(Z, \mathcal{O}_{Z}(m D)\right) \neq 0\right\}$, dim means the projective dimension and, for each $n, \phi_{|n D|}(Z)$ is the closure of the image of the rational map defined by the complete linear system $|n D|$. When $|n D| \neq \emptyset$, for some $n$, the Iitaka dimension satisfies $0 \leq \kappa(D) \leq \operatorname{dim}(Z)=2$. By definition, the Kodaira dimension of $Z$ is the Iitaka dimension of a canonical divisor on $Z$.

\subsection{Blowups and configurations}

A well-known tool in algebraic geometry is the concept of blowup. In this section we recall its definition in the case of surfaces and some of its properties that will be applied in later sections and chapters. We have mainly followed [9], [18], [22], [91] and [19]. We will keep the notations and conventions of the above section.

Let $Z_{0}$ be a smooth projective surface and $p$ a closed point of $Z_{0}$. Then, there exist a surface $Z$ and a morphism $\pi: Z \rightarrow Z_{0}$, which are unique up to isomorphism, such that 
(1) The restriction of $\pi$ to $\pi^{-1}\left(Z_{0} \backslash\{p\}\right)$ is an isomorphism onto $Z_{0} \backslash\{p\}$.

(2) $\pi^{-1}(p)=E$ is isomorphic to $\mathbb{P}^{1}$.

We say that $\pi$ is the blowup of $Z_{0}$ at $p$ and $E$ is the exceptional divisor (or exceptional curve) of the blowup (see, for instance, [9, Chapter II, Section II.1]).

In the literature the above definition often corresponds to the concept of monoidal transformation (see [71, Chapter V, Section 3]) to distinguish it from other more general transformations, and the surface $Z$ is denoted by $\mathrm{Bl}_{p}\left(Z_{0}\right)$.

Let $D$ be an effective divisor on $Z_{0}$. We call strict transform of $D$ on $Z$ to the divisor defined by the closure of $\pi^{-1}(D \backslash\{p\})$ on $Z$ and we denote it by $\tilde{D}$. The total transform of $D$ on $Z$ is the pull back $\pi^{*} D$ of $D$. It will be usually denoted by $D^{*}$. If $D_{1}$ and $D_{2}$ are two effective divisors on $Z_{0}$ which are linearly equivalent, then $D_{1}^{*}$ and $D_{2}^{*}$ are also linearly equivalent (see [73, Proposition 2.15]).

Some properties related to the blowup concept are the following.

Proposition 1.2.1. [9, Chapter II, Lemma II.2 and Proposition II.3] Let $Z_{0}$ be a surface, $Z$ the surface created by a blowup $\pi$ at a closed point $p \in Z_{0}$ and $E$ the exceptional divisor.

(a) Set $D$ an effective divisor on $Z_{0}$. It holds that $D^{*}=\tilde{D}+\operatorname{mult}_{p}\left(\varphi_{D}\right) E$.

(b) There is an isomorphism $\operatorname{Pic}\left(Z_{0}\right) \oplus \mathbb{Z} \cong \operatorname{Pic}(Z)$ given by $(D, m) \mapsto D^{*}+m E$.

(c) Let $D_{1}$ and $D_{2}$ be divisors on $Z_{0}$. Then $D_{1}^{*} \cdot D_{2}^{*}=D_{1} \cdot D_{2}, D_{1}^{*} \cdot E=0$ and $E^{2}=-1$.

(d) $K_{Z} \sim K_{Z_{0}}^{*}+E$, where $K_{Z}$ and $K_{Z_{0}}$ are canonical divisors on $Z$ and $Z_{0}$, respectively.

Now we are going to consider finite sequences of blowups at closed points and recall some concepts associate to them. As we will see, the above results can be also extended to these sequences.

Let $Z_{0}$ be a surface and $p$ a closed point of $Z_{0}$. The set of closed points in the exceptional divisor created after blowing up $p$ is called the first infinitesimal neighbourhood of $p$. By induction, if $i>1$, the $i$-th infinitesimal neighbourhood of $p$ is defined to be the first infinitesimal neighbourhood of some point in the $(i-1)$-th infinitesimal neighbourhood of $p$. A point $q$ is infinitely near $p$ when $q$ belongs to some $i$-th infinitesimal neighbourhood of $p$ for $i \geq 1$. The points which are infinitely near some point of $Z_{0}$ are called infinitely near $Z_{0}$. Occasionally, the closed points of $Z_{0}$ will be named proper in order to distinguish from the infinitely near ones.

Let $p$ and $q$ be two points which are proper or infinitely near $Z_{0}$. It is said that $p$ precedes $q$, denoted $p<q$, if and only if $q$ is infinitely near $p$. We write $p \leq q$ if $p$ equals or precedes $q$. The level of $p$, written $\mathrm{l}(p)$, is the number of proper or 
infinitely near points which precede $p$. The proper points of $Z_{0}$ are the points of level zero.

Consider a finite, or infinite, set $\mathcal{C}$ of proper and infinitely near $Z_{0}$ points. This set is said to be a configuration of infinitely near points (or configuration for short) over $Z_{0}$ if, for every point $q \in \mathcal{C}$, the points which precede $q$ belong to $\mathcal{C}$. The points of level zero of $\mathcal{C}$ are called origins of $\mathcal{C}$. When there exists a unique origin $p$ in $\mathcal{C}$, we will say that $\mathcal{C}$ is a configuration with origin at $p$. Unless otherwise stated, we only consider finite configurations.

The relation $\leq$ is a partial order for the set of elements of a configuration $\mathcal{C}$. Usually this relation is called natural order. If the natural order is a total order, then we say that the configuration $\mathcal{C}$ is a chain. Set $\preceq$ a total order on a configuration $\mathcal{C}$. The total order $\preceq$ is called admissible for any $p, q \in \mathcal{C}, p \leq q$ implies $p \preceq q$.

Fixed a configuration of infinitely near points $\mathcal{C}$, we can set $\mathcal{C}=\left\{p_{i}\right\}_{i=1}^{n}$ under an admissible total order such that $p_{1} \preceq p_{2} \preceq \ldots \preceq p_{n}$. Moreover, we can attach the pair $(\mathcal{C}, \preceq)$ a unique sequence of points blowups,

$$
\pi_{\mathcal{C}, \preceq}: Z=Z_{n} \stackrel{\pi_{n}}{\longrightarrow} Z_{n-1} \rightarrow \cdots \rightarrow Z_{1} \stackrel{\pi_{1}}{\longrightarrow} Z_{0} .
$$

such that $p_{i} \in Z_{i-1}$ and $Z_{i}=\mathrm{Bl}_{p_{i}}\left(Z_{i-1}\right)$, for $1 \leq i \leq n$. The surface $Z$ is called the sky of the configuration.

The sky of a configuration is eventually independent of the admissible total order as we see in the following result (see [22, Proposition 4.3.2] or [91, Proposition 1.2.4]).

Proposition 1.2.2. Assume that $\preceq$ and $\preceq^{\prime}$ are two admissible total orders of a same configuration of infinitely near points $\mathcal{C}$ over a surface $Z_{0}$. Set $Z$ and $Z^{\prime}$ the skies of the configuration associated to $\preceq$ and $\preceq^{\prime}$, respectively. Then there exists a unique isomorphism $\phi: Z \rightarrow Z^{\prime}$.

From now on, we consider configurations over a surface $Z_{0}$ endowed with an admissible total order and denote the corresponding sequence of blowups by $\pi$.

Let $\mathcal{C}=\left\{p_{i}\right\}_{i=1}^{n}$ be a configuration and consider

$$
\pi: Z=Z_{n} \stackrel{\pi_{n}}{\rightarrow} Z_{n-1} \rightarrow \cdots \rightarrow Z_{1} \stackrel{\pi_{1}}{\rightarrow} Z_{0}
$$

its sequence of blowups. The exceptional divisor created by blowing-up $p_{i}$ will be denoted $E_{p_{i}}$ (or simply $E_{i}$ when no confusion arises).

Let $D$ be a divisor on one of the surfaces in (1.1), $Z_{i}$. Abusing of notation, the strict (respectively, total) transform of $D$ on $Z_{j}$, for $j \geq i$, is denoted by $\tilde{D}$ (respectively, $D^{*}$ ). Frequently, the strict transform of $E_{i}$ will be simply written $E_{i}$. Also, we denote by $\operatorname{mult}_{p_{j}}\left(\varphi_{D}\right)$ the multiplicity of the strict transform of $D$ at the point $p_{i} \in \mathcal{C}, 1 \leq i \leq n$.

A point $p_{i}$ in $\mathcal{C}$ is proximate to a point $p_{j} \in \mathcal{C}, i>j$, when the point $p_{i}$ belongs to the strict transform of $E_{j}$ on $Z_{i-1}$. It is denoted by $p_{i} \rightarrow p_{j}$. A point $p_{i} \in \mathcal{C}$ is called 
satellite if $p_{i}$ is proximate to two points of $\mathcal{C}$. Otherwise, $p_{i}$ is named free. When each point $p_{i}$ belongs to the exceptional divisor $E_{i-1}$, for $2 \leq i \leq n$, (that is, when $\mathcal{C}$ is a chain) the sequence $\pi$ is said to be simple. Notice that, when $\mathcal{C}$ is a chain, a point $p_{i} \in \mathcal{C}$ is satellite if there exists an integer $j<i-1$ such that $p_{i}$ is proximate to $p_{j}$.

Proposition 1.2.3. [18, Theorem 1.6] Let $\mathcal{C}$ be a configuration of infinitely near points and $p, q$ and $r$ points in $\mathcal{C}$. The relation of proximity on $\mathcal{C}$ satisfies the following properties:

(a) If $q \rightarrow p$ then $p<q$.

(b) If $p \leq q$ and $l(q)=l(p)+1$ then $q \rightarrow p$.

(c) If $p<q \leq r$ and $r \rightarrow p$ then $q \rightarrow p$.

The following results considers a sequence of blowups as (1.1).

Proposition 1.2.4. [5, Proposition 1.1.26] Keep the notation introduced in the last paragraphs, the following statements hold.

(a) Set $D$ an effective divisor on $Z_{0}$. Then $D^{*}=\tilde{D}+\sum_{i=1}^{n} \operatorname{mult}_{p_{i}}\left(\varphi_{D}\right) E_{i}^{*}$.

(b) There exists an isomorphism $\operatorname{Pic}\left(Z_{0}\right) \oplus \mathbb{Z}^{n} \cong \operatorname{Pic}(Z)$ given by

$$
\left(D, m_{1}, m_{2}, \ldots, m_{n}\right) \mapsto D^{*}+\sum_{i=1}^{n} m_{i} E^{*} .
$$

(c) $K_{Z} \sim K_{Z_{0}}^{*}+\sum_{i=1}^{n} E_{i}^{*}$, where $K_{Z}$ and $K_{Z_{0}}$ are canonical divisors on $Z$ and $Z_{0}$, respectively.

(d) Set $D_{1}$ and $D_{2}$ two divisors on $Z_{0}$. Then it holds that

$$
D_{1}^{*} \cdot D_{2}^{*}=D_{1} \cdot D_{2}, D_{1} \cdot E_{i}^{*}=0 \text { and } D_{1}^{*} \cdot E_{i}=0, \text { for } 1 \leq i \leq n \text {. }
$$

(e) For all $i, j \in\{1,2, \ldots, n\}$

$$
E_{i} \cdot E_{j}=\left\{\begin{array}{cl}
-r_{i}-1 & \text { if } i=j, \\
1 & \text { if } i \neq j \text { and } E_{i} \cap E_{j} \neq \emptyset \\
0 & \text { otherwise, }
\end{array}\right.
$$

where $r_{i}$ is the number of points that are proximate to $p_{i}$.

(f) For all $i, j \in\{1,2, \ldots, n\}$

$$
E_{i}^{*} \cdot E_{j}^{*}=\left\{\begin{array}{cl}
-1 & \text { if } i=j, \\
0 & \text { otherwise. }
\end{array}\right.
$$


(g) For all $i, j \in\{1,2, \ldots, n\}$

$$
E_{i} \cdot E_{j}^{*}=\left\{\begin{array}{cl}
-1 & \text { if } i=j \\
1 & \text { if } p_{i} \rightarrow p_{j} \\
0 & \text { otherwise }
\end{array}\right.
$$

(h) If $D_{1}$ is a divisor on $Z_{0}$ and $D_{2}$ is a divisor on $Z$ then $D_{1}^{*} \cdot D_{2}=D_{1} \cdot \pi_{*} D_{2}$, where $\pi_{*} D_{2}$ is the direct image of the divisor $D_{2}$ on $Z_{0}$ induced by $\pi$.

Proposition 1.2.4 (a) gives the following expression with respect to the strict and total transforms of the exceptional divisors:

$$
E_{i}=E_{i}^{*}-\sum_{p_{j} \rightarrow p_{i}} E_{j}^{*}
$$

As a consequence, the set $\left\{E_{1}^{*}, E_{2}^{*}, \ldots, E_{n}^{*}\right\}$ is a $\mathbb{Z}$-basis of the free abelian group $\mathbb{Z} E_{1} \oplus \mathbb{Z} E_{2} \oplus \cdots \oplus \mathbb{Z} E_{n}$ of the divisors with exceptional support. The matrix $\mathbf{P}_{\mathcal{C}}:=$ $\left(p_{i j}\right)_{1 \leq i, j \leq n}$, where $p_{i j}=-E_{i} \cdot E_{j}^{*}$, is called proximity matrix of $\mathcal{C}$ and gives the change of basis between $\left\{E_{i}\right\}_{i=1}^{n}$ and $\left\{E_{i}^{*}\right\}_{i=1}^{n}$. In addition, for a curve $C$ it holds that

$$
\operatorname{mult}_{p_{i}}\left(\varphi_{C}\right)=\sum_{p_{j} \rightarrow p_{i}} \operatorname{mult}_{p_{j}}\left(\varphi_{C}\right)
$$

See [22, Theorem 3.5.3 (Proximity equalities)].

We conclude the section stating the concept of dual graph of a configuration over a surface $Z_{0}$.

A vertex-weighted graph is a pair $\left(\Gamma, \ell_{\Gamma}\right)$, where $\Gamma$ is a non-directed graph whose set of vertices is $V_{\Gamma}$ and $\ell_{\Gamma}: V_{\Gamma} \rightarrow \mathbb{Z}$ is a map. For simplicity, we will denote it by $\Gamma$. Set $\Gamma_{1}$ and $\Gamma_{2}$ two vertex-weighted graphs. A graph homomorphism $\left(\Gamma_{1}, \ell_{\Gamma_{1}}\right) \rightarrow\left(\Gamma_{2}, \ell_{\Gamma_{2}}\right)$ of vertex-weighted graphs is a morphism $\phi: \Gamma_{1} \rightarrow \Gamma_{2}$ such that $\ell_{\Gamma_{2}}(\phi(\mathbf{v}))=\ell_{\Gamma_{1}}(\mathbf{v})$ for all $\mathbf{v} \in V_{\Gamma_{1}}$.

Let $\Omega$ be a set $\left\{C_{1}, C_{2}, \ldots, C_{m}\right\}$ of integral curves on a surface $Z$. The dual graph of $\Omega$, denoted by $\Gamma(\Omega)$, is a vertex-weighted graph $\left(\Gamma(\Omega), \ell_{\Gamma(\Omega)}\right)$ obtained as follows. Its set of vertices corresponds one-to-one to the curves of $\Omega$ (that is, one assigns a vertex $\mathbf{v}(C)$ of $\Gamma(\Omega)$ to each curve $C \in \Omega)$; two vertices $\mathbf{v}\left(C_{i}\right)$ and $\mathbf{v}\left(C_{j}\right)$ are connected by an edge when $i \neq j$ and $C_{i} \cap C_{j} \neq \emptyset$; and $\ell_{\Gamma(\Omega)}\left(\mathbf{v}\left(C_{i}\right)\right):=C_{i}^{2}$.

Set $\mathcal{C}$ a configuration over a surface $Z_{0}$. The dual graph $\Gamma_{\mathcal{C}}$ of $\mathcal{C}$ is defined to be the dual graph of $\Omega:=\left\{E_{p}\right\}_{p \in \mathcal{C}}$.

\subsection{Plane valuations}

In this section we describe some properties and objects related to valuations of quotient fields of 2-dimensional regular local rings centered at them. As we will see, these valuations are directly connected to simple sequences of points blowups. Moreover, 
we recall some of their invariants, which are key objects in this dissertation. We have developed this section following [117], [109] and [36]. We will keep the notation as above.

Let $K$ be the quotient field of a 2-dimensional regular local ring $(R, \mathfrak{m})$ and set $K^{*}=K \backslash\{0\}$. Assume that $k$ is the residue field $R / \mathfrak{m}$ and it is algebraically closed and contained in $R$. Set $G$ a totally ordered abelian group. A valuation of $K$ is a surjective map $\nu: K^{*} \rightarrow G$ that satisfies:

$$
\nu(f+g) \geq \min \{\nu(f), \nu(g)\} \text { and } \nu(f g)=\nu(f)+\nu(g) \text {, for } f, g \in K^{*} .
$$

The group $G$ is called the value group of $\nu$. Given two valuations, $\nu_{1}: K^{*} \rightarrow G_{1}$ and $\nu_{2}: K^{*} \rightarrow G_{2}, \nu_{1}$ and $\nu_{2}$ are equivalent if there exists an order-preserving isomorphism $h: G_{1} \rightarrow G_{2}$ of their groups of values such that $h \circ \nu_{1}=\nu_{2}$ (see [117, Chapter VI, Section 8]).

The valuation ring of $\nu$ is the local regular ring $R_{\nu}:=\left\{f \in K^{*} \mid \nu(f) \geq 0\right\} \cup\{0\}$. Its maximal ideal is $\mathfrak{m}_{\nu}=\left\{f \in K^{*} \mid \nu(f)>0\right\} \cup\{0\}$. A valuation $\nu$ is said to be centered at $R$ when $R \cap \mathfrak{m}_{\nu}=\mathfrak{m}$. Two valuations centered at $R$ are equivalent if and only if their valuation rings are isomorphic [117, Chapter VI, Section 8].

The valuations of $K$ centered at $R$ are usually called plane valuations. Valuations (not only plane valuations) were central objects in some works of the middle of the last century by Abhyankar and Zariski which aim to solve problems of resolution of singularities in algebraic geometry (see [114, 115, 117, 1, 2] for example). Zariski introduced three classical invariants for valuations which, in the plane case, help us to classify them: the rank of $\nu$, which is denoted by $\operatorname{rk}(\nu)$ and defined as the Krull dimension of $R_{\nu}$; the rational rank $\operatorname{r.rk}(\nu)$ of $\nu$, which is the dimension of the $\mathbb{Q}$ vector space $G \otimes_{\mathbb{Z}} \mathbb{Q}$; and the transcendence degree of $\nu$, written $\operatorname{tr} \cdot \operatorname{deg}(\nu)$ and defined as the transcendence degree of the field extension $k_{\nu} / k$, where $k_{\nu}$ is the residue field $k_{\nu}:=R_{\nu} / \mathfrak{m}_{\nu}$. Abhyankar in [2] showed that (in our plane case) the above invariants satisfy the following inequalities:

$$
\operatorname{rk}(\nu)+\operatorname{tr} \cdot \operatorname{deg}(\nu) \leq \operatorname{r.rk}(\nu)+\operatorname{tr} \cdot \operatorname{deg}(\nu) \leq \operatorname{dim}(R)=2 .
$$

An important geometrical property of the plane valuations is given in the next theorem (see [117] and [109]).

Keep the above notation and let $\nu$ be a plane valuation. Set $\pi$ the (non-necessarily finite) simple sequence of point blowups:

$$
\pi: \cdots \rightarrow Z_{n} \stackrel{\pi_{n}}{\longrightarrow} Z_{n-1} \rightarrow \cdots \rightarrow Z_{1} \stackrel{\pi_{1}}{\rightarrow} Z_{0}=\operatorname{Spec} R,
$$

where $\pi_{1}$ is the blowup at the closed point $p:=p_{1} \in Z_{0}$ defined by the maximal ideal $\mathfrak{m}$ and $\pi_{i+1}, i \geq 1$, is the blowup at the unique closed point $p_{i+1}$ that belongs to the exceptional divisor $E_{i}$ created by $\pi_{i}$ and such that the plane valuation $\nu$ is centered at $\mathcal{O}_{Z_{i}, p_{i+1}}$.

The above assignation gives rise to the following result. 
Theorem 1.3.1. Plane valuations (up to equivalence) of $K$ centered at $R$ correspond one-to-one with sequences of point blowups as (1.3).

Let $\nu$ be a plane valuation. The semigroup of $\nu, S_{\nu}$, is defined to be the monoid $\nu(R \backslash\{0\}) \cup\{0\}$, and the set $\mathcal{C}_{\nu}=\left\{p_{1}, p_{2}, \ldots\right\}$ is called the configuration of infinitely near points of $\nu$. Following Section 1.2, notice that $\mathcal{C}_{\nu}$ is a configuration over $Z_{0}$ with origin at $p$ whose cardinality might be infinite. From now on, an exceptional divisor $E_{i}$ is called satellite (respectively, free) if the point $p_{i}$ is satellite (respectively, free).

Another useful object related to a plane valuation $\nu$ is its dual graph $\Gamma_{\nu}$, which is the dual graph of the configuration $\mathcal{C}_{\nu}$. Here, for simplicity, the weight attached to each vertex will be the positive integer $i$ which represents the exceptional divisor $E_{i}$, instead of the self-intersection of $E_{i}$. Notice that both ways of attaching weights to the graph are equivalent. A vertex of $\Gamma_{\nu}$ is called to be satellite (respectively, free) when it represents a satellite (respectively, free) exceptional divisor. As the sequence of blowups corresponding to $\nu$ is simple, the dual graph $\Gamma_{\nu}$ is a tree and the degree of the vertices of $\Gamma_{\nu}$ may be $0,1,2$ or 3 .

Let $\Gamma_{\nu}$ be the dual graph of a plane valuation $\nu$ and $\mathcal{C}_{\nu}$ the configuration of $\nu$. Next we introduce some notation. Set $s t_{0}:=1$ and let us define $g \in \mathbb{Z}_{\geq 0} \cup\{\infty\}$ and an increasing sequence of $g$ weights of $\Gamma_{\nu} s t_{1}, s t_{2}, \ldots$ distinguishing three cases: (1) $\mathcal{C}_{\nu}$ is infinite and $\Gamma_{\nu}$ has infinitely many vertices of degree $3,(2) \mathcal{C}_{\nu}$ is finite and its last point $p_{n}$ is satellite, and (3) otherwise. In case (1) we define $g:=+\infty$ and we set $s t_{1}<s t_{2}<\cdots$ the weights of the vertices of degree 3 . In case (2) $g-1$ is defined to be the number of vertices of degree 3 in $\Gamma_{\nu}$ and we set $s t_{1}<s t_{2}<\cdots<s t_{g-1}$ the weights of those vertices (if there are any) and $s t_{g}$ the weight of the vertex associated to $E_{p_{n}}$. In case (3) $g$ is the number of vertices of degree 3 in $\Gamma_{\nu}$ and set $s t_{1}<s t_{2}<\cdots<s t_{g}$ the weights of those vertices (if there are any). The vertices with weight $s t_{i}$ before introduced are usually called star vertices or stars.

In the above cases (2) and (3), we distinguish two subgraphs of $\Gamma_{\nu}: \hat{\Gamma}_{\nu}$ and $\Gamma_{\nu}^{g+1}$. The subgraph $\hat{\Gamma}_{\nu}$ is a connected graph formed by the vertices $\alpha$ such that $\alpha \leq s t_{g}$ and the edges which join them. The subgraph $\Gamma_{\nu}^{g+1}$ contains those vertices $\alpha$ such that $\alpha \geq s t_{g}$ and their attached edges. In case (1) we set $\hat{\Gamma}_{\nu}:=\Gamma_{\nu}$

Notice that $\hat{\Gamma}_{\nu}$ is just the vertex 1 when $g=0$. Otherwise, one can find more than one vertex with degree 1 in $\hat{\Gamma}_{\nu}$. These vertices are named dead ends and denoted in a ordered way by $\ell_{i}, 0 \leq i \leq g$. Thus, $\ell_{0}:=\mathbf{1}$.

In this work, we will use the following partial order on the set of the vertices of $\hat{\Gamma}_{\nu}$ : given two vertices $\alpha$ and $\beta$, we define $\alpha \preccurlyeq \beta$ if there exists a path from 1 to $\beta$ going through $\alpha$. This ordering allows us to divide $\hat{\Gamma}_{\nu}$ into subgraphs $\Gamma_{\nu}^{i}, 1 \leq i \leq g$, where $\Gamma_{\nu}^{i}$ contains the vertices $\alpha$ such that $s t_{i-1} \preccurlyeq \alpha \preccurlyeq \ell_{i}$ and the attached edges.

Plane valuations have been classified by Spivakovsky in five types according to their dual graphs (see [109]). Next we recall this classification. The names of the different types of valuations come from [47]. 


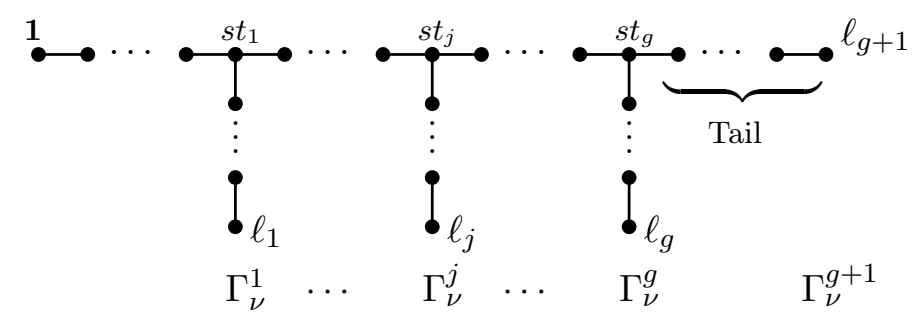

Figure 1.1: Dual graph of a divisorial valuation

A plane valuation $\nu$ is called divisorial if its configuration of infinitely near points is finite. We set $\ell_{g+1}$ the weight the last vertex. The subgraph of $\Gamma_{\nu}$ whose vertices (together with the edges joining them) are those whose weights $\alpha$ satisfy $s t_{g} \preccurlyeq \alpha \preccurlyeq$ $\ell_{g+1}$ is denoted $\Gamma_{\nu}^{g+1}$ and named tail. Note that $s t_{g}=\ell_{g+1}$ and it has degree 0 or 2 when the tail has a unique vertex. If $g=0$, every vertex of $\Gamma_{\nu}^{g+1}$ is free. Otherwise, all the vertices of $\Gamma_{\nu}^{g+1}$ are free with the exception of $s t_{g}$. Figure 1.1 shows the shape of $\Gamma_{\nu}$.

Up to isomorphism, the group of values of a divisorial valuation $\nu$ is $\mathbb{Z}$. When we consider this group embedded into $\mathbb{R}$, then there exists $c \in \mathbb{R} \backslash\{0\}$ such that $\nu(f)=c \cdot \operatorname{ord}_{E}(f)$ for all $f \in K^{*}$, where $E$ is the last exceptional divisor created by the sequence of point blowups associated to $\nu$ [109, Remark 2.7]. Different constants $c$ give rise to equivalent divisorial valuations defined by the same exceptional divisor. Throughout this work, we usually assume $c=1$. The equivalent valuation to $\nu$ given by $c=1 / \nu(\mathfrak{m})$, where $\nu(\mathfrak{m})=\min \{\nu(f) \mid f \in \mathfrak{m} \backslash\{0\}\}$, is called the normalization of $\nu$ and it is denoted $\nu^{N}$.

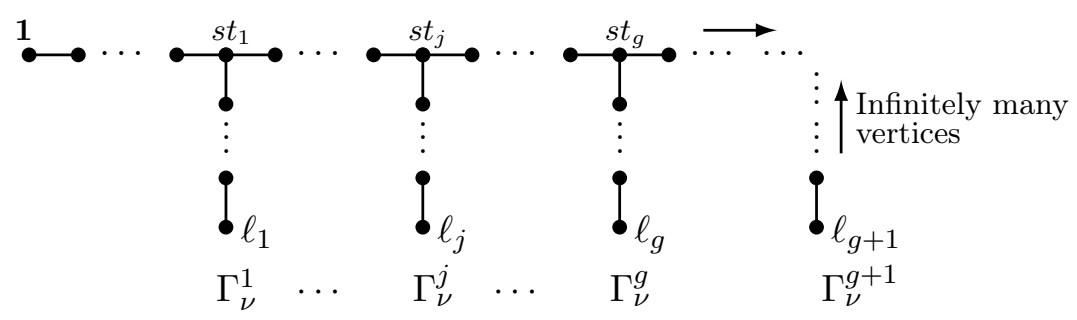

Figure 1.2: Dual graph of an irrational valuation

A valuation $\nu$ is named irrational when its configuration is infinite and there is a positive integer $r$ such that the points $p_{i}$, for $i \geq r$, are satellite and they are not proximate to a same point. Its group of values is a subgroup of $\mathbb{R}$. The dual graph $\Gamma_{\nu}$ is like that in Figure 1.2. The subgraph $\Gamma_{\nu}^{g+1}$ contains infinitely many vertices, the 
exceptional divisors corresponding to those vertices with weight $s t_{g}+1 \leq i \leq l_{g+1}$ are free and the remaining ones are satellite. Here, the vertex with weight $s t_{g}$ is always a star vertex if $g \neq 0$.

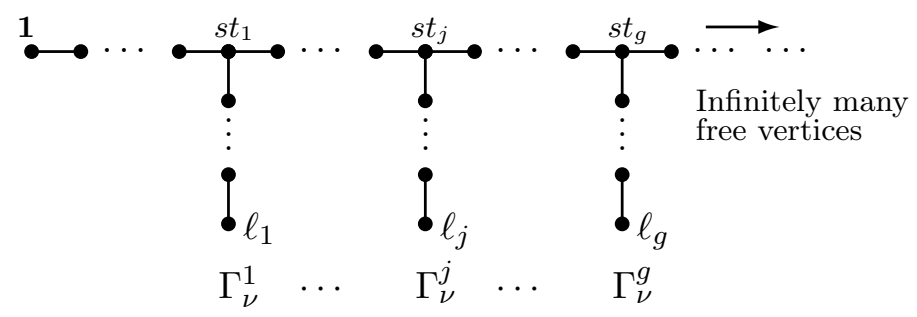

Figure 1.3: Dual graph of a non-exceptional curve valuation

A plane valuation is a non-exceptional curve valuation if its configuration contains infinitely many points and all of them are free after a point $p_{r}$. The group of values is isomorphic to $\mathbb{Z}_{\text {lex }}^{2}$ (lexicographically ordered). The dual graph associated to this type of valuations is depicted in Figure 1.3. In this case $\Gamma_{\nu}^{g+1}$ has infinitely many free vertices with the exception of $s t_{g}$ when $g \neq 0$.

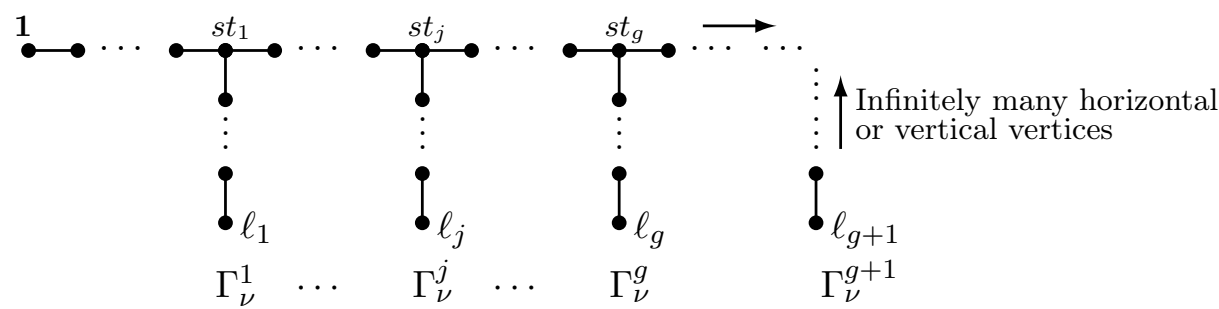

Figure 1.4: Dual graph of an exceptional curve valuation

An exceptional curve valuation is a plane valuation whose configuration has infinitely many points and there exists a point $p_{r}$ such that all the points $p_{i}$, where $i>r$, are proximate to $p_{r}$. $\mathbb{Z}_{\text {lex }}^{2}$ is the corresponding group of values. Its dual graph can be seen in Figure 1.4. The subgraph $\Gamma_{\nu}^{g+1}$ contains infinitely many vertices such that at the beginning a finite number of them are free and the remaining ones are satellite. Here, $s t_{g}$ is also the weight of a star vertex if $g \neq 0$ and $\ell_{g+1}$ is the last free vertex in $\Gamma_{\nu}^{g+1}$.

Finally, a plane valuation is called to be infinitely singular if its associated configuration is infinite and there are infinitely many points which give rise to divisors $E_{s t_{i}}$. That is, its dual graph (Figure 1.5) has an infinite number of star vertices. Its group of values is a subgroup of $\mathbb{R}$. 


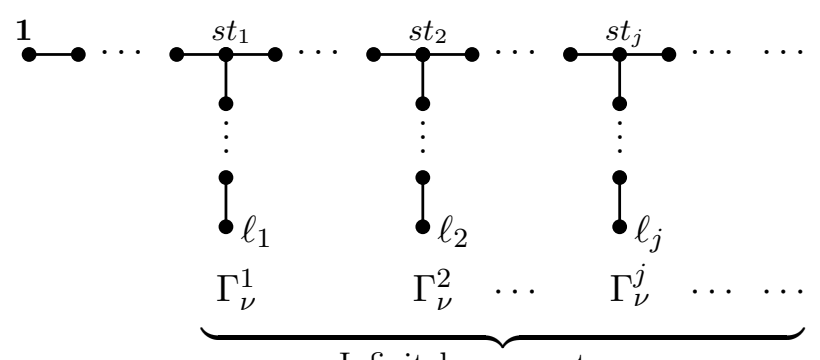

Infinitely many stars

Figure 1.5: Dual graph of infinitely singular valuation

The following result is useful to perform computations with non-divisorial valuations. More details can be found in [54] and [65, Section 3.2].

Theorem 1.3.2. Let $\mathcal{O}_{Z_{0}, p}$ be a regular local ring.

(a) Let $\nu$ be a non-exceptional curve valuation centered at $\mathcal{O}_{Z_{0}, p}$. There exists an analytically irreducible germ of curve $\varphi_{C}$ passing through $p$ such that

$$
\nu(f)= \begin{cases}\left(0,\left(\varphi_{C}, f\right)_{p}\right), & \text { if } \varphi_{C} \nmid f \\ \left(\alpha,\left(\varphi_{C}, f\right)_{p}\right), & \text { if } f=\varphi_{C}^{\alpha} h ; \varphi_{C} \nmid h\end{cases}
$$

where $f, h \in \mathcal{O}_{Z_{0}, p} \backslash\{0\}$.

(b) Let $\nu$ be an exceptional curve valuation centered at $\mathcal{O}_{Z_{0}, p}$, and $\mathcal{C}_{\nu}=\left\{p_{1}, p_{2}, \ldots\right\}$ its configuration where all points $p_{i}$ are proximate to a point $p_{r}$ for $i>r \geq 1$. Then, up to equivalence,

$$
\nu(f)=\left(v_{1}(f), v_{2}(f)\right), \text { for all } f \in \mathcal{O}_{Z_{0}, p} \backslash\{0\},
$$

where $v_{1}(f):=\nu_{r}(f)$ is the divisorial valuation defined by the exceptional divisors $E_{r}$ and $v_{2}(f)=\nu_{\eta}(f)+\sum_{p_{i} \rightarrow p_{r}}$ mult $_{p_{i}}(f), \nu_{\eta}$ being the divisorial valuation defined by $E_{\eta}$ and $\eta$ the index different from $r$ such that $p_{r+1} \in E_{\eta}$ when $p_{r+1}$ is satellite; otherwise, $\nu_{\eta}(f)=0$.

(c) Let $\nu$ be an irrational valuation centered at $\mathcal{O}_{Z_{0}, p}$. Then, up to equivalence,

$$
\nu(f)=\lim _{i \rightarrow \infty} \nu_{i}^{N}(f), \text { for all } f \in \mathcal{O}_{Z_{0}, p} \backslash\{0\},
$$

where $\nu_{i}^{N}$ is the normalization of the divisorial valuation defined by the exceptional divisor $E_{i}$ created by the sequence of points blowups corresponding to $\nu$.

(d) Let $\nu$ be an infinitely singular valuation centered at $\mathcal{O}_{Z_{0}, p}$. Then, up to equivalence,

$$
\nu(f)=\lim _{i \rightarrow \infty} \nu_{i}^{N}(f), \text { for all } f \in \mathcal{O}_{Z_{0}, p} \backslash\{0\},
$$

where $\nu_{i}^{N}$ is defined as above. 
Notice that the irrational and infinitely singular valuations of the above theorem are normalized. In this dissertation we always suppose that condition.

To conclude, in Figure 1.6 we show the relation of the above classification of the plane valuations with the Zariski invariants.

\begin{tabular}{|c|c|c|c|}
\hline Type & $\operatorname{rk}(\nu)$ & $\operatorname{rrrk}(\nu)$ & $\operatorname{tr} \cdot \operatorname{deg}(\nu)$ \\
\hline Divisorial valuation & 1 & 1 & 1 \\
\hline Irrational valuation & 1 & 2 & 0 \\
\hline Non-exceptional curve valuation & 2 & 2 & 0 \\
\hline Exceptional curve valuation & 2 & 2 & 0 \\
\hline Infinitely singular valuation & 1 & 1 & 0 \\
\hline
\end{tabular}

Figure 1.6: Table of the types of plane valuations

\subsubsection{More invariants of plane valuations}

Several invariants have been considered to study plane valuations. We define some of them for divisorial, irrational and exceptional curve valuations, since we will only consider these valuations in the following chapters (see [109, 36] for further information).

Let $\nu$ be a divisorial, irrational or exceptional curve valuation and $\mathcal{C}_{\nu}=\left\{p_{i}\right\}_{i \geq 1}$ $\left(p:=p_{1}\right)$ its configuration of infinitely near points. Write $\mathfrak{m}_{i}$ the maximal ideal corresponding to the point $p_{i}$ for $i \geq 1$. We call sequence of values of $\nu$ to the set $\left\{\nu\left(\mathfrak{m}_{i}\right)\right\}_{i \geq 1}$, where $\nu\left(\mathfrak{m}_{i}\right)=\min \left\{\nu(f) \mid f \in \mathfrak{m}_{i} \backslash\{0\}\right\}$. The sequence of values satisfies the proximities equalities [22, Theorem 8.1.7]:

$$
\nu\left(\mathfrak{m}_{i}\right)=\sum_{p_{j} \rightarrow p_{i}} \nu\left(\mathfrak{m}_{j}\right), i \geq 1
$$

when the set $\left\{p_{j} \in \mathcal{C}_{\nu} \mid p_{j} \rightarrow p_{i}\right\}$ is not empty. If $\nu$ is an exceptional curve valuation and $p_{i} \rightarrow p_{r}$ for every $i>r$, then $\nu\left(\mathfrak{m}_{r}\right)=(a, b)$ and $\nu\left(\mathfrak{m}_{i}\right)=(0, c)$, for some $a, b, c \in \mathbb{Z}, a, c>0$.

Denote by $\pi$ the simple sequence of blowups associated to $\nu$ as showed in (1.3). Following [109, Section 7] and [47, Chapter 6, Section 6, Subsection 1], there exists an analytically irreducible germ $\varphi_{i}$ at $p$ such that its strict transform on $Z_{i}$ is transversal to $E_{i}$ at any previously fixed non-singular point of the exceptional locus. In the divisorial case, it holds that $\nu\left(\mathfrak{m}_{i}\right)=\operatorname{mult}_{p_{i}}\left(\varphi_{n}\right)$, for $i \geq 1$.

Along this work we will often use the so-called Noether formula for valuations. One can find a proof in [22, Theorem 8.1.6]:

Lemma 1.3.3. Let $\nu$ be a divisorial, irrational or exceptional curve valuation whose configuration (of infinitely near points) is $\mathcal{C}_{\nu}=\left\{p_{i}\right\}_{i \geq 1}\left(p:=p_{1}\right)$. Let $C$ be a curve 
on $Z_{0}$. Then

$$
\nu\left(\varphi_{C}\right)=\sum_{i \geq 1} \nu\left(\mathfrak{m}_{i}\right) \cdot \operatorname{mult}_{p_{i}}\left(\varphi_{C}\right)
$$

As a consequence of the above result, if $\nu$ is a divisorial valuation defined by an exceptional divisor $E_{n}$, one has that, under a suitable choice of $\varphi_{n}, \nu\left(\varphi_{C}\right)=$ $\left(\varphi_{n}, \varphi_{C}\right)_{p}$.

The sequence of Puiseux exponents of $\nu$ is defined to be the ordered set $\left\{\beta_{i}^{\prime}(\nu)\right\}_{i=0}^{g+1}$ where $\beta_{0}^{\prime}(\nu)=1$ and, for $1 \leq i \leq g+1, \beta_{i}^{\prime}(\nu)$ is the continued fraction

$$
\beta_{i}^{\prime}(\nu)=a_{1}^{i}+\frac{1}{a_{2}^{i}+\frac{1}{\ddots+\frac{1}{a_{r_{i}}^{i}}}}=\left\langle a_{1}^{i} ; a_{2}^{i}, \ldots, a_{r_{i}}^{i}\right\rangle
$$

where $a_{k}^{i}, 1 \leq k<r_{i}+1$, successively counts the number of vertices in $\Gamma_{\nu}^{i}$ with the same value $\nu\left(\mathfrak{m}_{j}\right)$. It holds that $\beta_{i}^{\prime}(\nu) \in \mathbb{Q}_{>0} \backslash \mathbb{Z}_{>0}$, for $1 \leq i<g$, and they are bigger than 1. When $\nu$ is divisorial (respectively, irrational), then $r_{g+1}=1$ (respectively, $r_{g+1}=\infty$ ) and thus $\beta_{g+1}^{\prime}(\nu)$ is a positive integer (respectively, $\beta_{g+1}^{\prime}(\nu) \in \mathbb{R}_{>0} \backslash \mathbb{Q}_{>0}$ ). When $\nu$ is an exceptional curve valuation, then $a_{r_{g+1}}^{g+1}=\infty$ and so $\beta_{g+1}^{\prime}(\nu)$ does not exist (see [36] for more information).

Finally, the sequence of maximal contact values of $\nu$ is the set $\left\{\bar{\beta}_{i}(\nu)\right\}_{i=0}^{g+1}$, where each element $\bar{\beta}_{i}(\nu)$ is the value $\nu\left(\varphi_{\ell_{i}}\right)$ for $0 \leq i \leq g+1$. This family of invariants generates the semigroup of values $S_{\nu}$ of $\nu$. Moreover, if $\nu$ is a divisorial valuation defined by the divisor $E_{n}, \bar{\beta}_{g+1}(\nu)$ can be obtained as a combination of the remaining maximal contact values and satisfies

$$
\bar{\beta}_{g+1}(\nu)=\sum_{i=1}^{n} \nu\left(\mathfrak{m}_{i}\right)^{2},
$$

by the Noether formula.

Before stating an useful result, following [46, Section 5.2], we introduce an algorithm that extends the Euclidean division and the greatest common divisor for some values in the additive semigroup $\mathbb{R}_{\geq 0}^{n}$, where $n \geq 1$, under the lexicographical order. We denote the elements of $\mathbb{R}^{n}$ by $\bar{x}=\left(x_{1}, x_{2}, \ldots, x_{n}\right)$.

Proposition 1.3.4. [46, Proposition 5.13] Let $\bar{y} \leq \bar{x} \in \mathbb{R}_{\geq 0}^{n}$ be such that there exists an index $t(1 \leq t \leq n)$ satisfying $x_{j}=y_{j}=0$ for $j<t$ and $y_{t}>0$. Then there exists a unique positive integer $m$ such that $\bar{x}=m \bar{y}+\bar{z}$ and $(0,0, \ldots, 0)=: \overline{0} \leq \bar{z}<\bar{y}$.

As a consequence, given two elements $\bar{x}_{0}$ and $\bar{x}_{1}$ in $\mathbb{R}_{\geq 0}^{n}$ such that $\bar{x}_{1} \leq \bar{x}_{0}$, performing when possible "Euclidean divisions" as described in Proposition 1.3.4, 
one gets the following algorithm:

$$
\begin{array}{cccc}
\bar{x}_{0} & = & m_{0} \bar{x}_{1}+\bar{x}_{2} ; & \overline{0}<\bar{x}_{2}<\bar{x}_{1} \\
\bar{x}_{1} & = & m_{1} \bar{x}_{2}+\bar{x}_{3} ; & \overline{0}<\bar{x}_{3}<\bar{x}_{2} \\
\vdots & \vdots & \vdots & \vdots \\
\bar{x}_{l-1} & = & m_{l-1} \bar{x}_{l}+\bar{x}_{l+1} ; & \overline{0}<\bar{x}_{l+1}<\bar{x}_{l}
\end{array}
$$

The next possibilities for the algorithm can happen:

(1) It stops and $\bar{x}_{k}=m_{k} \bar{x}_{k+1}+\overline{0}$ holds for some index $k$.

(2) It never stops and one obtains an infinite sequence of non-negative integers $m_{l}, l \geq 0$.

(3) It stops and there exists, for some index $k$, another index $t(1 \leq t \leq n)$ such that the first $t$ components $x_{k+1, j}, 1 \leq j \leq t$, vanish, but $x_{k, t} \neq 0$, being $x_{k, 1}=\ldots=x_{k, t-1}=0$, that is, $m_{k}=\infty$.

When Item (1) holds, it is said that $\bar{x}_{k+1}$ is the greatest common divisor of $\bar{x}_{0}$ and $\bar{x}_{1}$, denoted by $\operatorname{gcd}\left(\bar{x}_{0}, \bar{x}_{1}\right)$.

Consider now the sequences of Puiseux exponents $\left\{\beta_{i}^{\prime}(\nu)\right\}_{i=0}^{g+1}$ and the maximal contact values $\left\{\bar{\beta}_{i}(\nu)\right\}_{i=0}^{g+1}$ of a divisorial, irrational or exceptional curve valuation. Then both sequences satisfy the following result [36, Lemma 1.8].

Proposition 1.3.5. Under the above notation, for $0 \leq i<g$,

$$
\bar{\beta}_{i+1}(\nu)=e_{i}(\nu)\left(\beta_{i+1}^{\prime}(\nu)-1\right)+n_{i}(\nu) \bar{\beta}_{i}(\nu)
$$

where $e_{i}(\nu):=\operatorname{gcd}\left(\bar{\beta}_{0}(\nu), \bar{\beta}_{1}(\nu), \ldots, \bar{\beta}_{i}(\nu)\right), n_{0}(\nu)=1$ and $n_{i}(\nu)=e_{i-1}(\nu) / e_{i}(\nu)$. The above formula is also true for $i=g$ when $\nu$ is a divisorial or irrational valuation.

As a result, it holds that $\bar{\beta}_{i}(\nu) \in \mathbb{Z}_{>0}, \bar{\beta}_{i+1}(\nu) \notin e_{i}(\nu) \mathbb{Z}$ and $\bar{\beta}_{i+1}(\nu)>n_{i}(\nu) \bar{\beta}_{i}(\nu)$, for $0 \leq i<g$. In addition, $\bar{\beta}_{g+1}(\nu) \geq n_{g}(\nu) \bar{\beta}_{g}(\nu)$ and, if $\nu$ is divisorial, $\bar{\beta}_{g+1}(\nu)$ is a positive integer.

The following results will be useful.

Corollary 1.3.6. Let $\nu$ be a divisorial valuation, $\left\{\beta_{i}^{\prime}(\nu)\right\}_{i=0}^{g+1}$ its sequence of Puiseux exponents and $\left\{\bar{\beta}_{i}(\nu)\right\}_{i=0}^{g+1}$ its sequence of maximal contact values. Define $e_{g}(\nu)=1$, $e_{i}(\nu)=\operatorname{gcd}\left(\bar{\beta}_{0}(\nu), \bar{\beta}_{1}(\nu), \ldots, \bar{\beta}_{i}(\nu)\right), n_{0}(\nu)=1, n_{i}(\nu)=e_{i-1}(\nu) / e_{i}(\nu)$, for $0 \leq i \leq g$, as in Proposition 1.3.5. Then

$$
\beta_{i}^{\prime}(\nu)=\frac{q_{i}(\nu)}{n_{i}(\nu)}, \text { for } 1 \leq i<g+1,
$$

for some $q_{i}(\nu) \in \mathbb{Z}_{>0}$ such that $\operatorname{gcd}\left(q_{i}(\nu), n_{i}(\nu)\right)=1$, and

$$
e_{i}(\nu)=\prod_{k=i+1}^{g} n_{k}(\nu), \text { for } 0 \leq i<g .
$$


Proof. Suppose $g=1$. It follows easily that $e_{0}(\nu)=\bar{\beta}_{0}(\nu)$ and $e_{1}(\nu)=1$. By Condition (1.5),

$$
\beta_{1}^{\prime}(\nu)=\frac{\bar{\beta}_{1}(\nu)}{e_{0}(\nu)}=\frac{\bar{\beta}_{1}(\nu) / e_{1}(\nu)}{e_{0}(\nu) / e_{1}(\nu)}=\frac{q_{1}(\nu)}{n_{1}(\nu)},
$$

where $q_{1}(\nu)$ and $n_{1}(\nu)$ are positive integers. Also, it holds that

$$
\operatorname{gcd}\left(q_{1}(\nu), n_{1}(\nu)\right)=\operatorname{gcd}\left(\bar{\beta}_{1}(\nu), \bar{\beta}_{0}(\nu)\right)=1,
$$

which completes the proof for the case $g=1$.

Now consider $g>1$. As above, by (1.5), one gets that

$$
\begin{aligned}
\beta_{i}^{\prime}(\nu) & =\frac{\bar{\beta}_{i}(\nu)-n_{i-1}(\nu) \bar{\beta}_{i-1}(\nu)+e_{i-1}(\nu)}{e_{i-1}(\nu)} \\
& =\frac{\left(\bar{\beta}_{i}(\nu)-n_{i-1}(\nu) \bar{\beta}_{i-1}(\nu)+e_{i-1}(\nu)\right) / e_{i}(\nu)}{e_{i-1}(\nu) / e_{i}(\nu)}=\frac{q_{i}(\nu)}{n_{i}(\nu)},
\end{aligned}
$$

for $i \in\{1,2, \ldots, g\}$, where $q_{i}(\nu)$ and $n_{i}(\nu)$ are positive integers satisfying that $\operatorname{gcd}\left(q_{i}(\nu), n_{i}(\nu)\right)=1$. Indeed, as $\bar{\beta}_{i-1}(\nu) \in e_{i-1}(\nu) \mathbb{Z}$ and $e_{i-2}(\nu), e_{i-1}(\nu), \bar{\beta}_{i-1}(\nu)$ and $\bar{\beta}_{i}(\nu) \in e_{i}(\nu) \mathbb{Z}$, it holds that $q_{i}(\nu)$ and $n_{i}(\nu)$ are positive integers. Now we prove that $\operatorname{gcd}\left(q_{i}(\nu), n_{i}(\nu)\right)=1$ reasoning by contradiction. Suppose that there exists a positive integer $m_{i}>1$ such that $\operatorname{gcd}\left(q_{i}(\nu), n_{i}(\nu)\right)=m_{i}$. Consequently, one obtains $\operatorname{gcd}\left(\bar{\beta}_{0}(\nu), \bar{\beta}_{1}(\nu), \ldots, \bar{\beta}_{i}(\nu)\right)=m_{i} e_{i}(\nu)$, which leads to a contradiction.

Finally, it holds that $e_{i}(\nu)=\prod_{k=i+1}^{g} n_{k}(\nu)$, where $n_{k}(\nu)=e_{k-1}(\nu) / e_{k}(\nu)$ and $0 \leq i<g$, since $e_{g}(\nu)=1$. This concludes the proof.

As a consequence of Proposition 1.3.5 and Corollary 1.3.6, the sequence of maximal contact values of a divisorial valuation $\nu$ might be computed inductively using exclusively its sequence of Puiseux exponents and taking $\bar{\beta}_{0}(\nu)=\prod_{i=1}^{g} n_{i}(\nu)$. Another immediate consequence of the above mentioned results is the following corollary.

Corollary 1.3.7. Let $\nu$ (respectively, $\nu^{\prime}$ ) be a divisorial valuation, $\left\{\beta_{i}^{\prime}(\nu)\right\}_{i=0}^{g+1}$ (respectively, $\left.\left\{\beta_{i}^{\prime}\left(\nu^{\prime}\right)\right\}_{i=0}^{\hat{g}+1}\right)$ its sequence of Puiseux exponents and $\left\{\bar{\beta}_{i}(\nu)\right\}_{i=0}^{g+1}$ (respectively, $\left.\left\{\bar{\beta}_{i}\left(\nu^{\prime}\right)\right\}_{i=0}^{\hat{g}+1}\right)$ its sequence of maximal contact values. Assume that $\hat{g} \leq g$. Consider the value $e_{i}(\nu)$ (respectively, $e_{i}\left(\nu^{\prime}\right)$ ) defined as in Corollary 1.3.6.

(a) If $\beta_{i}^{\prime}(\nu)=\beta_{i}^{\prime}\left(\nu^{\prime}\right)$ for $0 \leq i \leq k$, where $0 \leq k<\hat{g}$, then

$$
e_{i}\left(\nu^{\prime}\right)=e \cdot e_{i}(\nu) \text { and } \bar{\beta}_{i}\left(\nu^{\prime}\right)=e \cdot \bar{\beta}_{i}(\nu), \text { for } 0 \leq i \leq k,
$$

where $0 \leq k<\hat{g}$ and $e=e_{k}\left(\nu^{\prime}\right) / e_{k}(\nu)$.

(b) If $\beta_{i}^{\prime}(\nu)=\beta_{i}^{\prime}\left(\nu^{\prime}\right)$ for $0 \leq i \leq \hat{g}<g$, then

$$
e_{i}\left(\nu^{\prime}\right)=e \cdot e_{i}(\nu) \text { and } \bar{\beta}_{i}\left(\nu^{\prime}\right)=e \cdot \bar{\beta}_{i}(\nu), \text { for } 0 \leq i \leq \hat{g}<g,
$$

where $e=e_{\hat{g}}(\nu)^{-1}$.

(c) If $\beta_{i}^{\prime}(\nu)=\beta_{i}^{\prime}\left(\nu^{\prime}\right)$ for $0 \leq i \leq \hat{g}=g$, then

$$
e_{i}\left(\nu^{\prime}\right)=e_{i}(\nu) \text { and } \bar{\beta}_{i}\left(\nu^{\prime}\right)=\bar{\beta}_{i}(\nu), \text { for } 0 \leq i \leq \hat{g}=g .
$$




\subsection{Cones associated to a surface}

This section contains some basic concepts in convex analysis needed to study the cone of curves of a surface, one of the most important objects in this dissertation. In addition, we recall the definitions of several types of divisors, the convex cones that they generate and the connections among them. For the first part we have followed [53], [103] and [91] and for the second one [71] and [85]. We keep the notation established in the above sections.

\subsubsection{Convex cones}

Let $M \cong \mathbb{Z}^{m}$ be a free module of rank $m$ over $\mathbb{Z}$ and $N:=\operatorname{Hom}_{\mathbb{Z}}(M, \mathbb{Z})$ its dual module. Consider the $\mathbb{Z}$-bilinear pairing

$$
\langle,\rangle: N \times M \rightarrow \mathbb{Z}
$$

where $\langle\bar{y}, \bar{x}\rangle$ is the value of $\bar{y}$ in $\bar{x}$, for $\bar{x} \in M$ and $\bar{y} \in N$.

Set the vector space $V:=M \otimes_{\mathbb{Z}} \mathbb{R} \cong \mathbb{R}^{m}$ over $\mathbb{R}$ and its dual vector space $V^{*}:=N \otimes_{\mathbb{Z}} \mathbb{R} \cong \mathbb{R}^{m}$. Each element $\bar{x}$ of $M$ (respectively, $\bar{y}$ of $N$ ) can be identified with its image $\bar{x} \otimes 1$ in $V$ (respectively, $\bar{y} \otimes 1$ in $V^{*}$ ). We regard $M \subseteq V$ and $N \subseteq V^{*}$ and notice that the above pairing can be extended as follows:

$$
\langle,\rangle: V^{*} \times V \rightarrow \mathbb{R}
$$

Consider on $V$ and $V^{*}$ the induced usual topology of $\mathbb{R}^{m}$ and set $\|\cdot\|$ the associated metric on $V$ and $V^{*}$. Given $A, B$ subsets of $V$ (or $V^{*}$ ), denote by $\bar{A}$ the topological closure (with respect to the usual topology of $\mathbb{R}^{m}$ ), $\operatorname{Int}(A)$ the topological interior, $A^{\perp}$ the orthogonal complement of $A$ with respect to the bilinear pairing $\langle\rangle,$,$-A the$ set $-A=\{-a \mid a \in A\}$ and $A+B$ the set $A+B=\{a+b \mid a \in A$ and $b \in B\}$.

We also consider the vector spaces $V_{\mathbb{Q}}=M \otimes_{\mathbb{Z}} \mathbb{Q}$ and $V_{\mathbb{Q}}^{*}=N \otimes \mathbb{Q}$ over $\mathbb{Q}$. They can be identified as subsets of $V$ and $V^{*}$, respectively. We will denote by $A_{\mathbb{Q}}$ the intersection of a subset $A$ of $V\left(\right.$ respectively, $\left.V^{*}\right)$ and $V_{\mathbb{Q}}\left(\right.$ respectively, $\left.V_{\mathbb{Q}}^{*}\right)$.

Let $A$ be a subset of $V$. $A$ is said to be convex if $\lambda \bar{x}+(1-\lambda) \bar{y} \in A$, for $\bar{x}, \bar{y} \in A$ and $\lambda \in \mathbb{R}$ such that $0 \leq \lambda \leq 1$. The intersection of all convex sets containing $A$ is called the convex hull of $A$ and denoted by $\operatorname{conv}(A)$. Notice that this convex set is the smallest one which contains $A$.

Let $S$ be a non-empty subset of $V$. We say that $S$ is a convex cone (or simply a cone) if it satisfies the following conditions:

$$
\bar{x}+\bar{y} \in S \text { and } \alpha \bar{x} \in S \text {, for } \bar{x}, \bar{y} \in S \text { and } \alpha \in \mathbb{R}_{\geq 0} .
$$

The vector subspace of $V$ generated by $S$ is denoted by $\operatorname{lin}(S)$ and the dimension of $\operatorname{lin}(S)$ by $\operatorname{dim}(S)$. If $S=\{0\}$, the dimension is 0 . 
Notice that $\operatorname{Int}(S) \cup\{0\}, \bar{S}$ and the intersection of non-empty cones are cones. Indeed, set $\bar{x}, \bar{y} \in \operatorname{Int}(S) \cup\{0\}$ and we are going to show that $\bar{x}+\bar{y} \in \operatorname{Int}(S) \cup\{0\}$. We can suppose that $\bar{x}$ and $\bar{y}$ are different from 0 . There exist two neighbourhoods of 0 in $\operatorname{lin}(S), U$ and $V$, such that $\bar{x}+U \subset S$ and $\bar{y}+V \subset S$. Reasoning by contradiction, suppose that $\bar{x}+\bar{y} \notin \operatorname{Int}(S)$. Therefore, we can find an element $\bar{z} \in U \cap V$ such that $\bar{x}+\bar{y}+\bar{z} \notin S$. That is a contradiction, since $S$ is a cone. Set $\bar{x} \in \operatorname{Int}(S) \cup\{0\}$ and $\alpha \in \mathbb{R}_{\geq 0}$, let us show that $\alpha \bar{x} \in \operatorname{Int}(S) \cup\{0\}$. We can suppose that both $\alpha$ and $\bar{x}$ do not vanish. Then there exists a neighbourhood $U$ of 0 in $\operatorname{lin}(S)$ such that $\bar{x}+U \subset S$. Thus one has that $\alpha \bar{x} \in \operatorname{Int}(S)$ since otherwise, as $\alpha U$ is also a neighbourhood of 0 in $\operatorname{lin}(S), \alpha \bar{x}+\alpha \bar{z} \notin \operatorname{Int}(S)$ for some $\bar{z} \in U$, but $\alpha \bar{x}+\alpha \bar{z}=\alpha(\bar{x}+\bar{z}) \in S$ because $S$ is a cone and $\bar{x}+\bar{z} \in S$ and then one gets a contradiction. Similar arguments prove that $\bar{S}$ and the intersection of non-empty cones are cones.

Let $A$ be a non-empty subset of $V$. The family of cones which contain $A$ is non-empty, since $V$ is a cone. The intersection of all cones that contain $A$ is the smallest cone that contains $A$ and is denoted by $\operatorname{con}(A)$. When a cone $S$ of $V$ can be expressed as $S=\operatorname{con}(A)$, where $A$ is a non-empty subset of $V$, it is said that $S$ is generated by $A$.

A subset $A$ of $V$ is said to be polyhedral if it is the intersection of a finite number of closed semi-spaces. That is, if there exists $\bar{b}_{1}, \bar{b}_{2}, \ldots, \bar{b}_{k} \in V^{*}$ and $\beta_{1}, \beta_{2}, \ldots, \beta_{k} \in \mathbb{R}$ such that

$$
A=\left\{\bar{x} \in V \mid\left\langle\bar{b}_{i}, \bar{x}\right\rangle \geq \beta_{i} \text { for all } i=1,2, \ldots, k\right\} .
$$

If $A$ admits an expression like the previous one such that $\beta_{i}=0$ for all $1 \leq i \leq k$, it is said that $A$ is a polyhedral cone. Notice that every polyhedral set is a closed convex set.

A convex set $A$ in $V$ is said to be finitely generated if it can be expressed as $A=\operatorname{conv}\left(A_{1}\right)+\operatorname{con}\left(A_{2}\right)$, where $A_{1}$ and $A_{2}$ are two finite subsets of $V$.

One has the following property whose proof can be found in [103, Theorem 19.1].

Theorem 1.4.1. A set $A \subseteq V$ is polyhedral if and only if $A$ is a finitely generated convex set.

As a consequence of the above theorem, a polyhedral cone is a set $S$ of $V$ which can be defined as $S=\operatorname{con}(A)$, where $A$ is a finite subset of $V$.

Let $S$ be a cone in $V$. The cone $S$ is named rational if it is generated by elements in $M$. A rational cone $S \subset V$ is called regular when it is generated by a subset of a $\mathbb{Z}$-basis of $M$. If $S$ is a regular cone generated by a subset $\left\{\bar{e}_{1}, \bar{e}_{2}, \ldots, \bar{e}_{k}\right\}$ of a $\mathbb{Z}$-basis of $M$, any element of $S \cap M$ can be expressed as a unique positive linear combination of $\bar{e}_{1}, \bar{e}_{2}, \ldots, \bar{e}_{k}$.

The dual cone of a cone $S$ is defined to be the closed cone

$$
S^{\vee}:=\left\{\bar{y} \in V^{*} \mid\langle\bar{y}, \bar{x}\rangle \geq 0 \text { for all } \bar{x} \in S\right\} .
$$


If $S$ is a polyhedral cone, its dual cone is polyhedral (see [103, (Farkas' theorem) Chapter 19 and 22]. Moreover, $S=S^{\vee \vee}$ (see [103, Theorem 14.1]).

Proposition 1.4.2. Let $S \subset V$ be a regular cone generated by the $\mathbb{Z}$-basis $B=$ $\left\{\bar{e}_{i}\right\}_{i=1}^{m}$ of $\mathbb{Z}^{m}$. Then, $S^{\vee}$ is generated by the dual $\mathbb{Z}$-basis of $B, B^{*}=\left\{\bar{e}_{i}^{*}\right\}_{i=1}^{m}$.

Proof. Set $C^{*}$ the cone generated by $B^{*}$. It is clear that $C^{*} \subseteq C^{\vee}$ by the definition of $C^{*}$. We are going to prove that $C^{\vee} \subseteq C^{*}$. Let $\bar{x}$ be an element of $C^{\vee} \cap \mathbb{Z}^{m}$. As $B^{*}$ is the dual $\mathbb{Z}$-basis of $B$, one has that $\bar{x}=\sum_{i=1}^{m} \alpha_{i} \bar{e}_{i}^{*}$, where $\alpha_{i} \in \mathbb{Z}$, for $1 \leq i \leq m$. Then

$$
\alpha_{i}=\left\langle\bar{x}, \bar{e}_{i}\right\rangle \geq 0, \text { for } 1 \leq i \leq m,
$$

since $\bar{x}$ is an element of $C^{\vee}$. As a consequence, $\bar{x}$ can be expressed as a positive combination of the generators of $C^{*}$ and then it belongs to $C^{*}$.

Let $S$ be a polyhedral cone in $V$. The vector subspace of $V$ given by $S \cap(-S)$ is called the linearity space of $S$. By definition, the cone $S$ is strongly convex if it is closed and its linearity space is $\{0\}$.

There are some useful equivalent conditions to the fact of that a cone is strongly convex (see [53, Section 1.2]).

Proposition 1.4.3. Let $S$ be a polyhedral cone. Then, the following conditions are equivalent:

(a) $S$ is strongly convex.

(b) S contains no non-zero linear subspace.

(c) There is an element $\bar{q} \in S^{\vee}$ such that $S \cap\{\bar{q}\}^{\perp}=\{0\}$.

A subset $\mathcal{F}$ of a cone $S$ of $V$ is called a face of $S$ whenever $\mathcal{F}:=S \cap\{\bar{z}\}^{\perp}$, for some $\bar{z} \in S^{\vee}$. It holds that $S$ is a face of $S$, since $S=S \cap\{0\}^{\perp}$. If $S$ is a strongly convex cone, $\{0\}$ is also a face of $S$. Every face different from $S$ and $\{0\}$ is named proper face.

Some properties of the faces of a cone $S$ are shown in the next result.

Proposition 1.4.4. Let $S$ be a cone in $V$. The following conditions are satisfied:

(a) The faces of $S$ are cones in $V$.

(b) If $S$ is strongly convex, its faces are also strongly convex.

(c) If $S$ is polyhedral, its proper faces are also polyhedral cones.

(d) If $S$ is polyhedral, $S$ has a finite number of faces.

(e) If $\mathcal{F}_{1}$ and $\mathcal{F}_{2}$ are faces of $S$ such that $\mathcal{F}_{1} \subseteq \mathcal{F}_{2}$, then $\mathcal{F}_{1}$ is a face of $\mathcal{F}_{2}$. 
(f) If $S$ is polyhedral, $\mathcal{F}_{2}$ is a face of $S$ and $\mathcal{F}_{1}$ is a face of $\mathcal{F}_{2}$, then $\mathcal{F}_{1}$ is a face of $S$.

(g) If $S$ is polyhedral, then the map $\mathcal{F} \mapsto S^{\vee} \cap \mathcal{F}^{\perp}$ is a bijection between the set of the faces of $S$ and $S^{\vee}$. Moreover, if $\mathcal{F}_{1}$ and $\mathcal{F}_{2}$ are faces of $S$, it holds that $\operatorname{dim}(\mathcal{F})+\operatorname{dim}\left(S^{\vee} \cap \mathcal{F}^{\perp}\right)=\operatorname{dim}(V)$ and

$$
\mathcal{F}_{1} \subseteq \mathcal{F}_{2} \text { if and only if } S^{\vee} \cap \mathcal{F}_{2}^{\perp} \subseteq S^{\vee} \cap \mathcal{F}_{1}^{\perp}
$$

Proof. One obtains (a) as consequence of the definition of face.

To get (b) notice that, if $\mathcal{F}$ is a face of $S$, then $\mathcal{F} \cap(-\mathcal{F}) \subseteq S \cap(-S)$. As a result, if $S$ is strongly convex, the face $\mathcal{F}$ is strongly convex.

We are going to prove (c) and (d). As $S$ is a polyhedral cone, $S$ is the cone generated by a subset $\left\{\bar{v}_{1}, \bar{v}_{2}, \ldots, \bar{v}_{k}\right\}$ of $V$. Moreover, by definition, a face $\mathcal{F}$ of $S$ satisfies that $\mathcal{F}=S \cap\{\bar{q}\}^{\perp}$, where $\bar{q} \in S^{\vee}$, then $\mathcal{F}$ is the cone generated by those $\bar{v}_{i}$ such that $\left\langle\bar{q}, \bar{v}_{i}\right\rangle=0$. This fact completes the proof.

We also have (e) because if $\mathcal{F}_{1}=S \cap\{\bar{q}\}^{\perp}$, where $\bar{q} \in S^{\vee}$, then $\bar{q}$ belong to $\mathcal{F}_{2}^{\vee}$ by the definition of dual cone. Thus, $\mathcal{F}_{1}$ is a face of $\mathcal{F}_{2}$.

Finally, for proving (f) and (g) it sufficient to see [44, Proposition II.1.7] and [53, Section 1.2], respectively.

A face $\mathcal{F}$ of a cone $S$ is said to be extremal if, given two elements $\bar{z}_{1}, \bar{z}_{2} \in S \backslash\{0\}$ such that $\bar{z}_{1}+\bar{z}_{2} \in \mathcal{F}$, it holds that $\bar{z}_{1}, \bar{z}_{2} \in \mathcal{F}$. A 1-dimensional extremal face is an extremal ray. In addition, any polyhedral cone is the convex hull of its extremal rays [77, Definition 1.15].

We finish this first part of the section with the following result which allows us to compute the extremal rays of the dual cone of a cone in $V$. We only show a sketch of the proof which can be found in [91, Proposition A.3.22].

Proposition 1.4.5. Let $V$ be a vector space of dimension $m$ and $S$ a strongly convex polyhedral cone in $V$ such that $\operatorname{dim}(S)=m$. Set $R=\mathbb{R}_{\geq 0} \bar{r}=\left\{\alpha \bar{r} \mid \alpha \in \mathbb{R}_{\geq 0}\right\} \subset S^{\vee}$, where $\bar{r} \in V^{*}$. Then, $R$ is a extremal ray of $S^{\vee}$ if and only if there exist $m-1$ extremal rays $\mathcal{R}_{1}=\mathbb{R}_{\geq 0} \bar{r}_{1}, \mathcal{R}_{2}=\mathbb{R}_{\geq 0} \bar{r}_{2}, \ldots, \mathcal{R}_{m-1}=\mathbb{R}_{\geq 0} \bar{r}_{m-1}$ of $S$ such that $\left\{\bar{r}_{1}, \bar{r}_{2}, \ldots, \bar{r}_{m-1}\right\}$ are linearly independent vectors of $V$ and

$$
R=S^{\vee} \cap \mathcal{R}_{1}^{\perp} \cap \mathcal{R}_{2}^{\perp} \cap \cdots \cap \mathcal{R}_{m-1}^{\perp}
$$

Proof. Let $\mathcal{R}$ be an extremal ray of $S^{\vee}$. By Proposition 1.4.4, there exists a unique $(m-1)$-dimensional face $\mathcal{F}$ of $S$ such that $\mathcal{R}=S^{\vee} \cap \mathcal{F}^{\perp} \backslash\{0\}, \mathcal{F}$ being a strongly convex polyhedral cone generated by extremal rays of $S$ contained in $\mathcal{F}$. Denote by $\mathcal{R}_{1}, \mathcal{R}_{2}, \ldots, \mathcal{R}_{s}$ these rays. Since $\operatorname{dim}(\mathcal{F})=m-1$, there exist $i_{1}, i_{2}, \ldots, i_{m-1} \in$ $\{1,2, \ldots, s\}$ such that $\left\{\mathcal{R}_{i_{1}}, \mathcal{R}_{i_{2}}, \ldots, \mathcal{R}_{i_{m-1}}\right\}$ is a basis of $\operatorname{lin}(\mathcal{F})$. Therefore,

$$
\mathcal{F}^{\perp}=\operatorname{lin}(F)^{\perp}=\mathcal{R}_{i_{1}}^{\perp} \cap \mathcal{R}_{i_{2}}^{\perp} \cap \cdots \cap \mathcal{R}_{i_{m-1}}^{\perp} \text { and } \mathcal{R}=S^{\vee} \cap \mathcal{R}_{i_{1}}^{\perp} \cap \mathcal{R}_{i_{1}}^{\perp} \cap \cdots \cap \mathcal{R}_{i_{m-1}}^{\perp} .
$$


Now suppose that $R=\mathbb{R}_{\geq 0} \bar{r}$ is a subset of $S^{\vee}\left(\bar{r} \in V^{*}\right)$ such that

$$
\mathcal{R}=S^{\vee} \cap \mathcal{R}_{1}^{\perp} \cap \mathcal{R}_{2}^{\perp} \cap \cdots \cap \mathcal{R}_{m-1}^{\perp} \backslash\{0\},
$$

where $\mathcal{R}_{1}=\mathbb{R}_{\geq 0} \bar{r}_{1}, \mathcal{R}_{2}=\mathbb{R}_{\geq 0} \bar{r}_{2}, \ldots, \mathcal{R}_{m-1}=\mathbb{R}_{\geq 0} \bar{r}_{m-1}$ are extremal rays of $S$ and $\left\{\bar{r}_{1}, \bar{r}_{2}, \ldots, \bar{r}_{m-1}\right\}$ linearly independent vectors of $V$. Set $A$ the vector subspace of $V$ generated by $\left\{\bar{r}_{1}, \bar{r}_{2}, \ldots, \bar{r}_{m-1}\right\}$ and $\mathcal{F}:=S \cap A$. Given $\bar{z} \in \mathcal{R}$, it holds that $A=\{\bar{z}\}^{\perp}$ and $\mathcal{F}:=S \cap\{\bar{z}\}^{\perp}$. By definition of $\mathcal{F}, \bar{r}_{i}$ belongs to $\mathcal{F}$, for $1 \leq i \leq m-1$, and thus $\mathcal{F}$ is a $(m-1)$-dimensional face of $S$. As a result, $\mathcal{R}=S^{\vee} \cap \mathcal{F}^{\perp}$, since $\mathcal{F}^{\perp}=A^{\perp}=\mathcal{R}_{1}^{\perp} \cap \mathcal{R}_{2}^{\perp} \cap \cdots \cap \mathcal{R}_{m-1}^{\perp}$. Moreover, by Proposition 1.4.4 (e), $\mathcal{R}$ is a 1-dimensional face of $S^{\vee}$ and, consequently, it is an extremal ray of $S^{\vee}$.

\subsubsection{Cone of curves of a surface}

In this subsection we apply the above concepts and their properties to some geometric objects which we desire to study. We keep the notation introduced before.

Let $Z$ be a smooth projective surface over an algebraically closed field $k$ (surface for short). A divisor $D$ on $Z$ is numerically equivalent to zero, $D \equiv 0$, if $D \cdot C=0$ for every curve $C$ on $Z$. Two divisors $D_{1}$ and $D_{2}$ are numerically equivalent if $D_{1}-D_{2} \equiv 0$.

Recall that $\operatorname{Pic}(Z)$ is the Picard group of $Z$ and - the bilinear pairing associated to $\operatorname{Pic}(Z)$ (see Section 1.1 for more information). Inside $\operatorname{Pic}(Z)$ we can find the subgoup $\operatorname{Pic}^{\tau}(Z)$ given by the classes of the divisors numerically equivalent to zero and define $\operatorname{Num}(Z)$ as the set of classes of $\operatorname{Pic}(Z)$ modulo numerical equivalence, that is, $\operatorname{Num}(Z)=\operatorname{Pic}(Z) / \operatorname{Pic}^{\tau}(Z)$. As $Z$ is a smooth projective surface, the dual $\mathbb{Z}$-module $\operatorname{Hom}_{\mathbb{Z}}(\operatorname{Num}(Z), \mathbb{Z})$ of $\operatorname{Num}(Z)$ is isomorphic to $\operatorname{Num}(Z)$. Therefore, we have a bilinear pairing, $\operatorname{Num}(Z) \times \operatorname{Num}(Z) \rightarrow \mathbb{Z}$, associated to $\operatorname{Num}(Z)$, induced by the bilinear pairing associated to $\operatorname{Pic}(Z)$ (see [71, Chapter V, Remark 1.9.1]). As a consequence of the Nerón-Severi theorem [83], it holds that $\operatorname{Num}(Z)$ is a free $\mathbb{Z}$-module of finite rank. The rank of $\operatorname{Num}(Z)$ is named the Picard number of $Z$.

An element $D$ of the $\mathbb{Q}$-vector space $\operatorname{Div}_{\mathbb{Q}}(Z):=\operatorname{Div}(Z) \otimes \mathbb{Q}$ (R्R-vector space $\operatorname{Div}_{\mathbb{R}}(Z):=\operatorname{Div}(Z) \otimes \mathbb{R}$ ) is called $\mathbb{Q}$-divisor (respectively, $\mathbb{R}$-divisor). That is, $D$ can be expressed as

$$
D=n_{1} G_{1}+n_{2} G_{2}+\cdots+n_{r} G_{r},
$$

where $G_{i}$ is a divisor on $Z$ and $n_{i} \in \mathbb{Q}$ (respectively, $n_{i} \in \mathbb{R}$ ) for $1 \leq i \leq r$. A $\mathbb{Q}$ divisor (respectively, $\mathbb{R}$-divisor) is said to be effective when $G_{i}$ is effective and $n_{i} \geq 0$ for all $i$ and some of them is positive.

A $\mathbb{Q}$-divisor (respectively, $\mathbb{R}$-divisor) $D$ is numerically equivalent to zero, $D \equiv 0$, if $D \cdot C=0$ for every curve $C$ on $Z$. Two $\mathbb{Q}$-divisors (respectively, $\mathbb{R}$-divisors) $D_{1}$ and $D_{2}$ are numerically equivalent if $D_{1}-D_{2} \equiv 0$. The resulting vector space of numerical equivalence classes is denoted by $\operatorname{Num}_{\mathbb{Q}}(Z)\left(\right.$ respectively, $\left.\operatorname{Num}_{\mathbb{R}}(Z)\right)$ and 
one has the isomorphism $\operatorname{Num}_{\mathbb{Q}}(Z)=\operatorname{Num}(Z) \otimes_{\mathbb{Z}} \mathbb{Q}[85$, Remark 1.3.4] (respectively, $\operatorname{Num}_{\mathbb{R}}(Z)=\operatorname{Num}(Z) \otimes_{\mathbb{Z}} \mathbb{R}\left[85\right.$, Example 1.3.10]). The vector space $\operatorname{Num}(Z)_{\mathbb{R}}$ has finite dimension by Nerón-Severi theorem. Moreover, we can extend the bilinear pairing $\operatorname{Num}(Z) \times \operatorname{Num}(Z) \rightarrow \mathbb{Z}$ to a bilinear pairing $\operatorname{Num}_{\mathbb{R}}(Z) \times \operatorname{Num}_{\mathbb{R}}(Z) \rightarrow \mathbb{R}$ that, abusing of the notation, will also be denoted by $\cdot$. That is, given $\left[D_{1}\right],\left[D_{2}\right] \in$ $\operatorname{Num}_{\mathbb{R}}(Z),\left[D_{1}\right] \cdot\left[D_{2}\right] \in \mathbb{R}$. As a result, we have similar conditions to those introduced at the beginning of this section and then we can consider the concepts and results of convex cones within $\operatorname{Num}_{\mathbb{R}}(Z)$.

By definition, the cone of curves of $Z$, denoted by $\mathrm{NE}(Z)$, is the following convex cone in $\operatorname{Num}_{\mathbb{R}}(Z)$ :

$$
\operatorname{NE}(Z)=\left\{[D] \in \operatorname{Num}_{\mathbb{R}}(Z) \mid[D]=\sum_{i} a_{i}\left[C_{i}\right], \begin{array}{l}
\text { where } C_{i} \text { is an integral curve } \\
\text { on } Z \text { and } a_{i} \in \mathbb{R}_{\geq 0} .
\end{array}\right\}
$$

Its topological closure for the usual topology is denoted by $\overline{\mathrm{NE}}(Z)$ and, as $Z$ is a surface, the $\mathbb{R}$-divisors whose class belongs $\overline{\mathrm{NE}}(Z)$ are called pseudoeffective [85, Definition 2.2.25 and Remark 2.2.27].

Proposition 1.4.6. [77, Lemma 1.22] Let $C$ be an irreducible curve on a surface $Z$. If $C^{2} \leq 0$, then $[C]$ is in the boundary of $\overline{\mathrm{NE}}(Z)$. If $C^{2}<0$, then $[C]$ generates an extremal ray in $\mathrm{NE}(Z)$ and $\overline{\mathrm{NE}}(Z)$.

A divisor (respectively, $\mathbb{Q}$-divisor, $\mathbb{R}$-divisor) on $Z$ is said to be nef if

$$
D \cdot C \geq 0, \text { for every irreducible curve } C \text { on } Z \text {. }
$$

The convex cone containing the numerical equivalence classes of these $\mathbb{R}$-divisors is denoted by $\operatorname{Nef}(Z)$. The dual cone of the nef cone $\operatorname{Nef}(Z)$ is the convex cone $\overline{\operatorname{NE}}(Z)$ (see [85, Proposition 1.4.28]) and then a divisor $D$ is pseudoeffective if and only if $D \cdot D^{\prime} \geq 0$ for every nef divisor $D^{\prime}$. Moreover, it holds that $\operatorname{Nef}(Z) \subset \overline{\mathrm{NE}}(Z)[85$, Example 1.4.33 (i)], since $Z$ is a smooth projective surface.

We will also use ample and big divisors. A divisor $D$ which satisfies NakaiMoishezon criterion [71, Chapter V, Theorem 1.10], that is, $D^{2}>0$ and $D \cdot C>0$, for every irreducible curve $C$ on $Z$, is called to be ample. A divisor $D$ is named big if $\kappa(D)=\operatorname{dim} Z$. In [85, Corollary 2.2.7] one can find the following characterization of bigness for a divisor: A divisor $D$ is big if and only if there exist an ample divisor $H$, a positive integer $n$ and an effective divisor $G$ such that $n D \equiv H+G$.

One can find in [85, Theorem 2.2.16] the following characterization of bigness for a nef divisor.

Theorem 1.4.7. Let $D$ be a nef divisor on a surface $Z$. Then $D$ is big if and only if its self-intersection is strictly positive, i.e. $D^{2}>0$. 
A $\mathbb{Q}$-divisor (respectively, $\mathbb{R}$-divisor) $D$ is said to be ample if it can be written as

$$
D=a_{1} H_{1}+a_{2} H_{2}+\cdots+a_{r} H_{r},
$$

where $H_{i}$ is an ample divisor and $a_{i} \in \mathbb{Q}_{>0}$ (respectively, $a_{i} \in \mathbb{R}_{>0}$ ), for $1 \leq i \leq r$. A $\mathbb{Q}$-divisor $D$ is big if there is a positive integer $m>0$ such that $m D$ is integral and big. A $\mathbb{R}$-divisor $D$ is big if it can be written as

$$
D=a_{1} B_{1}+a_{2} B_{2}+\cdots+a_{r} B_{r}
$$

where, for all $i, B_{i}$ is a big integral divisor and $a_{i} \in \mathbb{R}_{>0}$. The convex cone generated by the numerical equivalence classes of ample (respectively, big) $\mathbb{R}$-divisors is denoted by $\operatorname{Amp}(Z)$ (respectively, $\operatorname{Big}(Z)$ ).

The following result gives us a relation among the convex cones above introduced. A proof can be found in [76] and [85, Theorem 1.4.23 and Theorem 2.2.26].

Theorem 1.4.8. Keeping the notation used before, it holds that:

(a) The nef cone $\operatorname{Nef}(Z)$ is the closure of the ample cone $\operatorname{Amp}(Z)$ and $\operatorname{Amp}(Z)$ is the interior of $\operatorname{Nef}(Z)$.

(b) The big cone $\operatorname{Big}(Z)$ is the interior of $\overline{\mathrm{NE}}(Z)$ and $\overline{\mathrm{NE}}(Z)$ is the closure of $\operatorname{Big}(Z)$.

Now we are going to introduce a last convex cone named characterisitic cone (see [76] for further information). The characteristic cone of a surface $Z$ is the convex cone of $\operatorname{Num}_{\mathbb{R}}(Z)$ generated by the numerical equivalence classes of the semiample divisors on $Z$, that is, those divisors $D$ such that the complete linear system $|m D|$ is base-point-free for some positive integer $m$. This convex cone is denoted $\tilde{P}(Z)$ and satisfies the following property.

Proposition 1.4.9. [76] With the previous notation, it holds that $\tilde{P}(Z) \subseteq \operatorname{Nef}(Z)$ and their interiors coincide.

To conclude this section, we present several results which we will use in the following chapters.

Theorem 1.4.10 (Kleiman's Ampleness Criterion). Let $Z$ be a surface. A divisor $D$ on $Z$ is ample if and only if $[D] \cdot\left[D^{\prime}\right]>0$ for all $\left[D^{\prime}\right] \in \overline{\mathrm{NE}}(Z) \backslash\{0\}$.

The above result was proved by Kleiman [76] and shows that $\overline{\mathrm{NE}}(Z)$ is a strongly convex cone.

Theorem 1.4.11. [71, Chapter V, Theorem 1.9 (Hodge index theorem)] Let $H$ be an ample divisor on a surface $Z$ and $D$ a divisor on $Z$ such that $D \not \equiv 0$ and $D \cdot H=0$. Then, $D^{2}<0$. 
A well-known consequence of Sylvester theorem (see [84, Chapter XV, Section 4, Theorem 4.1]) is that the bilinear form defined on the vector $\operatorname{space} \operatorname{Num}_{\mathbb{R}}(Z)$ can be diagonalized with only \pm 1 's on the diagonal. In addition, by Hodge index theorem (see [71, Chapter V, Remark 1.9.1]), the diagonalized intersection pairing only has one +1 corresponding to a multiple of an ample divisor $H$, and the remaining values on the diagonal are -1 . This allows us to state and prove the following result.

Proposition 1.4.12. Let $D$ be a big and nef divisor on a surface $Z$. Let $\Omega=$ $\left\{C_{1}, C_{2}, \ldots, C_{m}\right\}$ be a set of integral curves on $Z$ such that $D \cdot C_{i}=0$ for all $i=$ $1,2, \ldots, m$. Then the intersection matrix $\left(C_{i} \cdot C_{j}\right)$ is negative definite.

Proof. Let $M$ be the $m \times m$ matrix whose entries are $C_{i} \cdot C_{j}$. First notice that for every $\bar{y}=\left(y_{1}, y_{2}, \ldots, y_{m}\right) \in \mathbb{R}^{m} \backslash\{0\}$,

$$
\bar{y} M \bar{y}^{t}=G(\bar{y})^{2},
$$

where $G(\bar{y})$ is the $\mathbb{R}$-divisor $\sum_{i=1}^{m} y_{i} C_{i}$. Hence, it is enough to see that the selfintersection of any non-zero class in the hyperplane

$$
[D]^{\perp}:=\left\{\bar{x} \in \operatorname{Num}_{\mathbb{R}}(Z) \mid[D] \cdot \bar{x}=0\right\}
$$

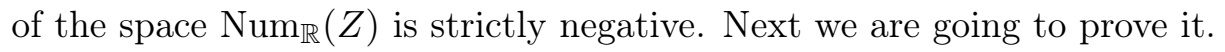

Set an ample divisor $H$ on $Z$. By Hodge index theorem (Theorem 1.4.11) there exists a basis $B=\left\{\mathbf{h}_{0}, \mathbf{h}_{1}, \ldots, \mathbf{h}_{n}\right\}$ of $\operatorname{Num}_{\mathbb{R}}(Z)$ such that $\mathbf{h}_{0}$ is a (real) multiple of $[H], \mathbf{h}_{0}^{2}=1, \mathbf{h}_{0} \cdot \mathbf{h}_{i}=0$ and $\mathbf{h}_{i} \cdot \mathbf{h}_{j}=-\delta_{i j}$ for all $i, j \in\{1,2, \ldots, n\}$, where $\delta_{i j}$ denotes the Kronecker delta. Since the intersection matrix whose entries are $\left(\mathbf{h}_{i} \cdot \mathbf{h}_{j}\right)_{1 \leq i, j \leq n}$ is negative definite, we can restrict our proof to those points of the hyperplane $[D]^{\perp}$ of $\operatorname{Num}_{\mathbb{R}}(Z)$ whose first coordinate with respect to $B$ is 1 . Let $\alpha_{1}, \alpha_{2}, \ldots, \alpha_{n} \in \mathbb{R}$ such that $\left(1, \alpha_{1}, \ldots, \alpha_{n}\right)$ are the coordinates of the vector $\frac{1}{D \cdot H}[D]$ with respect to the basis $B$. Since $D$ is nef and big, $D^{2}>0$ by Theorem 1.4 .7 and therefore

$$
\sum_{i=1}^{n} \alpha_{i}^{2}<1
$$

Now, an arbitrary element of $\operatorname{Num}_{\mathbb{R}}(Z)$ whose coordinates with respect to $B$ are

$$
\left(1, x_{1}, \ldots, x_{n}\right)
$$

belongs to $[D]^{\perp}$ if and only if

$$
\sum_{i=1}^{n} \alpha_{i} x_{i}=1,
$$

and has negative self-intersection if and only if $\sum_{i=1}^{n} x_{i}^{2}>1$.

Then our statement on the classes in $[D]^{\perp}$ follows because the map

$$
f: \mathbb{R}^{n} \rightarrow \mathbb{R}, \quad f\left(x_{1}, x_{2}, \ldots, x_{n}\right)=\sum_{i=1}^{n} x_{i}^{2}
$$


subject to the restriction (1.8), has an absolute minimum at the point

$$
\bar{p}=\frac{1}{\sum_{i=1}^{n} \alpha_{i}^{2}}\left(\alpha_{1}, \alpha_{2}, \ldots, \alpha_{n}\right) \text { and } f(\bar{p})=\frac{1}{\sum_{i=1}^{n} \alpha_{i}^{2}}>1 .
$$

Notice that $\bar{p}$ is the closest to the origin point of the hyperplane defined by the equation (1.8) and the last inequality follows from (1.7). This concludes the proof.

Proposition 1.4.13. [77, Corollary 1.21] Let $H$ be an ample divisor on a surface $Z$. The set $Q(Z):=\left\{[D] \in \operatorname{Num}_{\mathbb{R}}(Z) \mid[D]^{2}>0\right\}$ has two connected components

$Q^{+}(Z):=\{[D] \in Q(Z) \mid[D] \cdot[H]>0\}$ and $Q^{-}(Z):=\{[D] \in Q(Z) \mid[D] \cdot[H]<0\}$. In particular, $Q^{+}(Z) \subset \overline{\mathrm{NE}}(Z)$.

Proposition 1.4.14. Let $Z$ be a surface. Set $H$ an ample divisor on $Z$ and write

$$
A(Z):=\left\{[D] \in \operatorname{Num}_{\mathbb{R}}(Z) \mid[D]^{2} \geq 0 \text { and }[D] \cdot[H] \geq 0\right\} .
$$

Then, it holds that $A(Z)=A(Z)^{\vee}$, where $A(Z)^{\vee}$ is the dual cone of $A(Z)$.

Proof. As the proof of Proposition 1.4.12, assume that $B=\left\{\mathbf{h}_{0}, \mathbf{h}_{1}, \ldots, \mathbf{h}_{n}\right\}$ is a basis of $\operatorname{Num}_{\mathbb{R}}(Z)$ such that $\mathbf{h}_{0}^{2}=1, \mathbf{h}_{0} \cdot \mathbf{h}_{i}=0$ and $\mathbf{h}_{i} \cdot \mathbf{h}_{j}=-\delta_{i j}$, for $1 \leq i, j \leq n$, where $\delta_{i j}$ is the Kronecker delta. Denote by $\langle\cdot, \cdot\rangle$ and $\|\cdot\|$ the standard inner product in $\mathbb{R}^{n}$ and the Euclidean norm which it defines.

We start by showing that $A(Z) \subseteq A(Z)^{\vee}$. Fix $[D] \in A(Z) \backslash\{0\}$ whose coordinates in the basis $B$ are $\left(d_{0}, d_{1}, \ldots, d_{n}\right)$. Since $[D] \in A(Z) \backslash\{0\}$, it holds that $d_{0}>0$. Otherwise, $d_{0}=[D] \cdot \mathbf{h}_{0}=0,-\sum_{i=1}^{n} d_{i}^{2}=[D]^{2} \geq 0$ and then $d_{i}$ vanishes for all $i$. Set $\bar{x}=\left(x_{1}, x_{2}, \ldots, x_{n}\right) \in \mathbb{R}^{n}$ such that $x_{i}=d_{i} / d_{0}$, for $1 \leq i \leq n$. By definition, one has that $\|\bar{x}\| \leq 1$. Assume, reasoning by contradiction, that $[D] \notin A(Z)^{\vee}$. Consequently, one can find $\left[D^{\prime}\right] \in A(Z)$ with coordinates $\left(d_{0}^{\prime}, d_{1}^{\prime}, \ldots, d_{n}^{\prime}\right)$ in the basis $B$ such that $[D] \cdot\left[D^{\prime}\right]<0$. Write $\bar{y}=\left(y_{1}, y_{2} \ldots, y_{n}\right) \in \mathbb{R}^{n}$ such that $y_{i}=d_{i}^{\prime} / d_{0}^{\prime}$, for $1 \leq i \leq n$. One obtains that

$$
1<\sum_{i=1}^{n} x_{i} y_{i}=\langle\bar{x}, \bar{y}\rangle=|\langle\bar{x}, \bar{y}\rangle| \leq\|\bar{x}\|\|\bar{y}\| \leq 1 \cdot 1=1,
$$

where $|\cdot|$ is the absolute value and the second inequality is satisfied by CauchySchwarz inequality. That is a contradiction and then $[D] \in A(Z)^{\vee}$.

Now we are going to show that $A(Z)^{\vee} \subseteq A(Z)$, which will conclude the proof. Set $[D] \in A(Z)^{\vee} \backslash\{0\}$ with coordinates $\left(d_{0}, d_{1}, \ldots, d_{n}\right)$ in the basis $B$. Notice that $\mathbf{h}_{0}, \mathbf{h}_{0}+\mathbf{h}_{i}$ and $\mathbf{h}_{0}-\mathbf{h}_{i}$ belong to $A(Z)$ for all $i$. Hence, $d_{0}>0$ because, otherwise, $d_{0}=[D] \cdot \mathbf{h}_{0}=0, d_{i}=[D] \cdot\left(\mathbf{h}_{0}+\mathbf{h}_{i}\right) \geq 0,-d_{i}=[D] \cdot\left(\mathbf{h}_{0}-\mathbf{h}_{i}\right) \geq 0$ and then $d_{i}$ vanishes for all $i$. Reasoning by contradiction, suppose that $[D] \notin A(Z)$. Therefore, $[D]^{2}<0$. Set an element $\bar{x}=\left(x_{1}, x_{2}, \ldots, x_{n}\right) \in \mathbb{R}^{n}$, where $x_{i}=d_{i} / d_{0}$ for $i \in$ 
$\{1,2, \ldots, n\}$. Consequently, $\|\bar{x}\|>1$. Take a divisor $D^{\prime}$ whose class has coordinates $\left(1, x_{1} /\|\bar{x}\|, \ldots, x_{n} /\|\bar{x}\|\right)$ (with respect to $B$ ) and a vector $\bar{y}=\left(y_{1}, y_{2}, \ldots, y_{n}\right) \in \mathbb{R}^{n}$ such that $y_{i}=x_{i} /\|\bar{x}\|$, for $1 \leq i \leq n$. It holds that $\|\bar{y}\|=1,\left[D^{\prime}\right]^{2}=0$ and so the class of $D^{\prime}$ belongs to $A(Z)$. Then

$$
[D] \cdot\left[D^{\prime}\right] \geq 0 \text { and }\langle\bar{x}, \bar{y}\rangle \leq 1 .
$$

On the other hand, taking into account that $\bar{y}=\bar{x} /\|\bar{x}\|$,

$$
\|\bar{x}\|=\frac{1}{\|\bar{x}\|}\langle\bar{x}, \bar{x}\rangle=\langle\bar{x}, \bar{y}\rangle \leq 1
$$

holds, which is a contradiction.

\subsection{Seshadri-type constants and Newton-Okounkov bo- dies}

In this section we briefly introduce Seshadri-type constants and show their relation to Newton-Okounkov bodies corresponding to certain flags on surfaces. These bodies will be defined later and are convex sets of $\mathbb{R}^{2}$. Our development is supported on [85, Chapter 5], [8],[86], [82] [15], [40] and [38]. Here, we use the notation established before, although in this section $k=\mathbb{C}$.

\subsubsection{Seshadri-type constants of divisorial valuations}

Let $Z_{0}$ be a (complex smooth irreducible projective) surface and $D$ a nef divisor on $Z_{0}$. Let $\pi: Z \rightarrow Z_{0}$ be the blowup at a point $p \in Z_{0}$ with exceptional divisor $E$. The Seshadri constant of $D$ at $p$, denoted by $\varepsilon\left(Z_{0}, D ; p\right)$ (or $\varepsilon(D ; p)$ for short), is the non-negative real number

$$
\varepsilon(D ; p)=\sup \left\{t \in \mathbb{R}_{\geq 0} \mid D^{*}-t E \text { is nef on } Z\right\},
$$

where $D^{*}$ denotes the pull back $\pi^{*} D$.

This constant depends only on the numerical equivalence class of $D$ and satisfies the homogeneity property: $\varepsilon(n D ; p)=n \varepsilon(D ; p)$, for every positive integer $n$ and point $p \in Z_{0}$ [85, Examples 5.1.3 and 5.1.4].

Notice that the ray $\left[D^{*}\right]-t[E]$ meets the boundary of the nef cone of $Z$ for the value $t=\varepsilon(D ; p)$. Then, the Seshadri constant $\varepsilon(D ; p)$ provides information about the positivity at $p$ of the divisor $D$ [85, Remark 5.1.2].

An equivalent definition of the Seshadri constant is given in the following result. A proof can be found in [85, Proposition 5.1.5].

Proposition 1.5.1. Keep the notation introduced before. Then

$$
\varepsilon(D ; p)=\inf _{p \in C \subseteq Z_{0}}\left\{\frac{D \cdot C}{\operatorname{mult}_{p}\left(\varphi_{C}\right)}\right\},
$$

where the infimum is taken over all integral curves $C \subseteq Z_{0}$ going through $p$. 
The explicit computation of the Seshadri constants is very hard. However, there exist some upper and lower bounds given by several authors. Let us show some of them.

According [8], if $D$ is an ample divisor on a smooth projective surface $Z_{0}$, then

$$
\varepsilon(D, p) \leq \sqrt{D^{2}} .
$$

Even more, if the Seshadri constant $\varepsilon(D, p)$ is irrational, then the equality holds and, when the strict inequality is satisfied, $\varepsilon(D, p)$ is rational. In addition, it holds that $\varepsilon(D, p) \geq 1$, for all except countably many points $p \in Z_{0}$ [85, Proposition 5.2.3].

The Seshadri constants have not only been used to study deeply the positivity of divisors on surfaces, but also on varieties and, particularly, on projective spaces, see [85, Chapter 5] and [8] for more information.

Some invariants which contain similar information to Seshadri constants have been also introduced. Next we give some information about them.

Let $\mathcal{I} \subseteq \mathcal{O}_{Z_{0}}$ be an ideal sheaf and set $D$ an ample divisor on $Z_{0}$. Let $\pi: Z \rightarrow Z_{0}$ be the blowup of $Z_{0}$ along $\mathcal{I}$ whose exceptional divisor is denoted by $E$. The $s$ invariant of $\mathcal{I}$ with respect to $D$ is the positive real number

$$
s_{D}(\mathcal{I})=\inf \left\{t \in \mathbb{R} \mid t D^{*}-E \text { is nef on } Z\right\} .
$$

Notice that if we set the closed point $p \in Z_{0}$ defined by a maximal ideal $\mathfrak{m} \subseteq \mathcal{O}_{Z_{0}}$, then

$$
\varepsilon(D ; p)=1 / s_{D}(\mathfrak{m})
$$

The s-invariant of $\mathcal{I}$ with respect to $D$ is introduced in [31] to study the complexity of a polynomial ideal in terms of the degrees of its generators from a geometrical perspective.

Another important invariant related to the Seshadri constants is given in [40]. Let $D$ be an big divisor on $Z_{0}$. The value $\mu(D ; p)$ is defined to be

$$
\mu(D ; p):=\sup \left\{t \in \mathbb{R} \mid D^{*}-t E \text { is big on } Z\right\} .
$$

Note that the ray $\left[D^{*}\right]-t[E]$ meets the boundary of the pseudoeffective cone $\overline{\mathrm{NE}}(Z)$ for the value $t=\mu(D ; p)$.

In [40, Remark 2.1] a connection between the invariant $\mu(D ; p)$ and the Seshadri constant $\epsilon(D ; p)$ is established. Assume that $D$ is an ample divisor. If $\varepsilon(D ; p)$ is irrational, $\mu(D ; p)=\varepsilon(D ; p)$ holds and, when $\mu(D ; p)$ is rational, then so is $\varepsilon(D ; p)$. In addition, it is proved (see [40, Proposition 2.2]) that

$$
\mu(D ; p)=\limsup _{m \rightarrow \infty} \frac{\max \left\{\operatorname{ord}_{p}(f) \mid f \in H^{0}\left(Z_{0}, \mathcal{O}_{Z_{0}}(m D)\right)\right\}}{m} .
$$


The authors of [15] (see also [38] and [64]) extend the previous value $\mu(D ; p)$ from a point $p$ to a real valuation $\nu$ of the function field of $Z_{0}$ centered at the local ring $\mathcal{O}_{Z_{0}, p}$ and a big divisor $D$ on $Z_{0}$ giving rise to the value

$$
\hat{\mu}_{D}(\nu):=\lim _{m \rightarrow \infty} \frac{\max \left\{\nu(f) \mid f \in H^{0}\left(Z_{0}, \mathcal{O}_{Z_{0}}(m D)\right)\right\}}{m} .
$$

Furthermore, they prove that

$$
\hat{\mu}_{D}(\nu)=\sup \left\{t \in \mathbb{R} \mid D^{*}-t E \text { is big on } Z\right\},
$$

when $\nu$ is the divisorial valuation defined by an exceptional divisor $E$ on $Z$ [15, Theorem 2.24], and provide the following lower bound for a real valuation of the function field of $Z_{0}$ :

$$
\hat{\mu}_{D}(\nu) \geq \sqrt{\frac{\operatorname{vol}_{Z_{0}}(D)}{\operatorname{vol}(\nu)}},
$$

where, by [43], the value

$$
\operatorname{vol}(\nu):=\lim _{\alpha \rightarrow \infty} \frac{\operatorname{length}\left(\mathcal{O}_{Z_{0}, p} / \mathcal{P}_{\alpha}\right)}{\alpha^{2} / 2},
$$

$\mathcal{P}_{\alpha}$ being the ideal $\mathcal{P}_{\alpha}=\left\{f \in \mathcal{O}_{Z_{0}, p} \mid \nu(f) \geq \alpha\right\} \cup\{0\}$ of the local ring $\mathcal{O}_{Z_{0}, p}$ where $\nu$ is centered, is called the volume of $\nu$ and

$$
\operatorname{vol}_{Z_{0}}(D):=\limsup _{m \rightarrow \infty} \frac{h^{0}\left(Z_{0}, m D\right)}{m^{2} / 2}
$$

is named the volume of $D$.

The case when $Z_{0}=\mathbb{P}^{2}$ and $D=L$ a general projective line on $\mathbb{P}^{2}$ is studied in [38] and [64]. This value $\hat{\mu}_{L}(\nu)$ can be expressed as

$$
\hat{\mu}_{L}(\nu)=\lim _{m \rightarrow \infty} \frac{\max \left\{\nu(f) \mid f \in H^{0}\left(\mathbb{P}^{2}, \mathcal{O}_{\mathbb{P}^{2}}(m L)\right)\right\}}{m}
$$

and also can been seen geometrically as $\hat{\mu}_{L}(\nu)=\sup \left\{t \in \mathbb{R} \mid L^{*}-t E\right.$ is big on $\left.Z\right\}$, where $Z$ is the surface given by $\nu$ and $L^{*}$ the pull-back of $L$ on $Z$.

It is important to mention that $\hat{\mu}_{L}(\nu)$ is involved in a variation of the Nagata Conjecture in the valuative setting, which implies that conjecture (see [38] and [64] for more information).

\subsubsection{Newton-Okounkov bodies of big divisors on a surface}

In this subsection we introduce the Newton-Okounkov bodies corresponding to flags on surfaces. A general description of these bodies can be seen in the introduction of this dissertation (see page 4 ).

Let $Z$ be a smooth complex projective surface and $K(Z)$ its function field. A flag on $Z$ is a sequence

$$
C_{\bullet}:=\{Z \supset C \supset\{q\}\},
$$


where $C$ is a smooth irreducible curve on $Z$ and $q$ a closed point of $C$ named center of $C_{\bullet}$.

A flag $C_{\bullet}$ as above comes with a discrete valuation $\nu_{C}$ of rank 2 of the function field $K(Z)$ (see [28] for further information). Set $g=0$ the local equation at $q$ of $C$ on $Z$. This valuation $\nu_{C}: K(Z) \rightarrow \mathbb{Z}_{\text {lex }}^{2}$ can be computed as follows:

$$
\nu_{C}(f)=\left(v_{1}(f), v_{2}(f)\right), \text { where } v_{1}(f):=\operatorname{ord}_{C}(f) \text { and } v_{2}(f):=\operatorname{ord}_{q}\left(\frac{f}{g^{v_{1}(f)}}\right),
$$

for $f \in K(Z)$.

Let $C$. be a flag and $D$ a big divisor on $Z$. The Newton-Okounkov body of $D$ with respect to $C_{\bullet}$ is the subset of $\mathbb{R}^{2}$

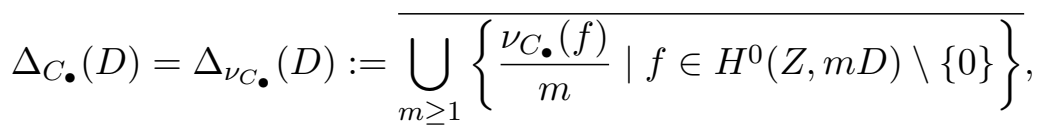

where the upper line means the closed convex hull in $\mathbb{R}^{2}$. This definition can be extended to big $\mathbb{Q}$-divisors and big $\mathbb{R}$-divisors (see [86]). In our case, $Z$ is a surface, the Newton-Okounkov body is a polygon (see [82]) and

$$
\operatorname{vol}_{Z}(D)=2 \operatorname{vol}_{\mathbb{R}^{2}}\left(\Delta_{\nu}(D)\right),
$$

where $\operatorname{vol}_{\mathbb{R}^{2}}$ means Euclidean area (see [86]) and $\operatorname{vol}_{Z}(D)>0$ since $D$ is big [85, Subsection 2.2.C]. In addition, Newton-Okounkov bodies satisfy the homothetic property, that is, $\Delta_{\nu}(d D)=d \cdot \Delta_{\nu}(D)$ for any integer $d>0$ (see [86, Proposition 4.1]).

Newton-Okounkov bodies for big $\mathbb{R}$-divisors on a surface can be described making use of the Zariski decomposition of these divisors.

Set $D$ a pseudoeffective (see page 35$) \mathbb{R}$-divisor on a surface $Z$. Then the divisor $D$ can be written as

$$
D=P_{D}+N_{D}
$$

where $N_{D}$ is an effective $\mathbb{R}$-divisor such that, if $N_{D} \neq 0$, their irreducible components generate a negative definite intersection matrix and $P_{D}$ is a nef $\mathbb{R}$-divisor orthogonal to $N_{D}$, i.e. $P_{D} \cdot N_{D}=0$ (see [116] and [52]). The above expression is called the Zariski decomposition of $D$, and $P_{D}$ (respectively, $N_{D}$ ) is the positive (respectively, negative) part of $D$.

An important consequence of the Zariski decomposition is that $\operatorname{vol}(D)=P_{D}^{2}[85$, Corollary 2.3.22].

Let $D$ be a big $\mathbb{R}$-divisor on a surface $Z$ and $C \subset Z$ an integral curve. Write

$$
\mu(D, C)=\sup \{t>0 \mid D-t C \text { is big }\} .
$$

Theorem 1.5.2. [86, Theorem 6.4] and [82, Theorem B] Keep the notation considered before. The Newton-Okounkov body $\Delta_{\nu_{C}}(D)$ of $D$ with respect to $\nu_{C}$. is the region

$$
\Delta_{\nu_{C}}(D)=\{(t, y) \mid 0 \leq t \leq \mu(D, C) \text { and } \alpha(t) \leq y \leq \beta(t)\}
$$


where $\alpha(t):=\operatorname{ord}_{q}\left(\left.N_{D_{t}}\right|_{C}\right)$ and $\beta(t):=\alpha(t)+P_{D_{t}} \cdot$ D. Here, $P_{D_{t}}$ (respectively, $N_{D_{t}}$ ) is the positive (respectively, negative) part of the divisor $D_{t}=D-t C$.

Let $\pi: Z \rightarrow Z_{0}$ be the blowup of a point $p \in Z_{0}$ with exceptional divisor $E$. Consider a big divisor $D$ on $Z_{0}$ such that $p$ does not belong to its support. Denote by $D^{*}$ the total transform (or the pull-back) of $D$ on $Z$. Then, by Equality (1.9),

$$
\mu\left(D^{*}, E\right)=\hat{\mu}_{D}(\nu)
$$

where $\nu$ is the divisorial valuation defined by $E$.

The Zariski decomposition of a big and nef divisor also allows us to provide a description of the big cone $\operatorname{Big}(Z)$ of a smooth projective surface $Z$ (see [7] for more information).

Let $D$ be a $\mathbb{R}$-divisor on $Z$ and $D=P_{D}+N_{D}$ its Zariski decomposition. We define the sets

$$
\operatorname{Null}(D)=\{C \mid C \text { is a irreducible curve with } D \cdot C=0\}
$$

and

$$
\operatorname{Neg}(D)=\left\{C \mid C \text { is a irreducible component of } N_{D}\right\} .
$$

Notice that $\operatorname{Neg}(D) \subseteq \operatorname{Null}\left(P_{D}\right)$ holds since $P_{D} \cdot N_{D}=0$.

Let $P$ be a big and nef divisor. The set $\Sigma_{P}$ defined as

$$
\Sigma_{P}:=\{[D] \in \operatorname{Big}(Z) \mid \operatorname{Neg}(D)=\operatorname{Null}(P)\}
$$

is called Zariski chamber. This set is a convex cone which, in general, is neither open nor closed. By [7, Proposition 1.6], the interior of $\Sigma_{P}$ is given by the set

$$
\left\{[D] \in \operatorname{Big}(Z) \mid \operatorname{Neg}(D)=\operatorname{Null}(P)=\operatorname{Null}\left(P_{D}\right)\right\} .
$$

In addition, a big divisor $D$ on $Z$ is in the boundary of some $\Sigma_{P}$ if and only if $\operatorname{Neg}(D) \neq \operatorname{Null}\left(P_{D}\right)$ (see [7, Proposition 1.5]).

Theorem 1.5.3. [7, Theorem 1.11] Let $Z$ be a smooth projective surface. Then there is a locally finite decomposition of the big cone of $Z$ into rational locally polyhedral subcones such that in each subcone the support of the negative part of the Zariski decomposition of the divisors is constant.

\subsection{Rational surfaces}

In this section we review the definition and some properties of Hirzebruch surfaces which will be fundamental in the next chapters. Our main references are [71], [9], [102] and [90]. We also show some other results about rational surfaces that can be 
found in [71, Chapter V, Section 5] and [9, Chapter II]. We maintain the notation of the previous sections.

Let $k$ be an algebraically closed field of arbitrary characteristic and $Z_{1}$ and $Z_{2}$ two smooth projective surfaces over $k$ (surfaces for short). Recall that a Zariski open subset of a surface is dense and, given two morphism $f$ and $g$ from $Z_{1}$ to $Z_{2}$ such that there is a non-empty open subset $U \subset Z_{1}$ satisfying $\left.f\right|_{U}=\left.g\right|_{U}$, then $f=g[71$, Chapter I, Lemma 4.1]. A rational map $f: Z_{1} \rightarrow Z_{2}$ is a morphism $f: U \rightarrow Z_{2}$ from a non-empty open subset $U \subset Z_{1}$ to $Y$. Two rational maps $f: U \rightarrow Z_{2}$ and $g: V \rightarrow Z_{2}$, where $U, V \subset Z_{1}$, are the same if $f$ and $g$ coincide on a non-empty open subset of $U \cap V$. A rational map $f: Z_{1} \rightarrow Z_{2}$ is said to be dominant if the image of $f$ contains a non-empty open subset $W$ of $Z_{2}$. In this case, if $g: Z_{2} \rightarrow Z_{3}$ is a rational map between surfaces defined on a non-empty open subset $V$ of $Z_{2}$, the composition $g \circ f: Z_{1} \rightarrow Z_{3}$ is defined on the non-empty subset $f^{-1}(V \cap W)$. A rational map $f$ is called to be birational when it is dominant and there exists another dominant rational map $g: Z_{2} \rightarrow Z_{1}$ such that $g \circ f=\operatorname{Id}_{Z_{1}}$ and $f \circ g=\operatorname{Id}_{Z_{2}}$ as birational morphism. In this last case, it is said that $Z_{1}$ and $Z_{2}$ are birational, or birationally equivalent. Notice that, by definition, two surfaces $Z_{1}$ and $Z_{2}$ are birational if and only if they contain isomorphic open subsets.

Let $Z$ be a surface. We will denote by $B(Z)$ the set of isomorphism classes of surfaces which are birationally equivalent to $Z$. A surface $Z$ is relatively minimal if its class in $B(Z)$ is minimal in the following sense: each birational morphism $Z \rightarrow Z^{\prime}$ is an isomorphism, where $Z^{\prime}$ is a surface which belongs to $B(Z)$.

Proposition 1.6.1. [9, Chapter II, Proposition II.16] For any surface $Z$, there is a birational morphism $Z \rightarrow Z^{\prime}$, where $Z^{\prime}$ is a relatively minimal surface.

Theorem 1.6.2. [9, Chapter II, Theorem II.11] Let $f: Z \rightarrow Z_{0}$ be a birational morphism of surfaces. Then, there is a sequence of blowups $\pi_{k}: Z_{k} \rightarrow Z_{k-1}$, where $1 \leq k \leq n$, and an isomorphism $u: Z \rightarrow Z_{n}$ such that $f=\pi_{1} \circ \ldots \circ \pi_{n} \circ u$.

These results can also be found in [71, Chapter V, Theorem 5.8 and Theorem 5.5, respectively]. As a consequence, a smooth projective surface can be obtained by a finite sequence of point blowups where the first one is centered at a point of a relatively minimal surface. We are going to show another characterization of these surfaces.

Let $C$ be a curve on a surface $Z$. $C$ is a $(-1)$-curve (or a exceptional curve of the first kind) whenever $C$ is isomorphic to $\mathbb{P}^{1}$ and $C^{2}=-1$. Notice that the exceptional divisor created by a blowup is a (-1)-curve.

The following result, called Castelnuovo's contractibility criterion, shows that a $(-1)$-curve on a surface is the exceptional divisor of a blowup.

Theorem 1.6.3. [71, Chapter V, Theorem 5.7] Let $Z$ be a surface containing a $(-1)$-curve $C$. Then, there exist a morphism $f: Z \rightarrow Z^{\prime}$ between surfaces, a closed 
point $p \in Z^{\prime}$ and an isomorphism $u: Z \rightarrow B l_{p}\left(Z^{\prime}\right)$ such that $u(C)$ is the exceptional divisor of the blowup $\pi: B l_{p}\left(Z^{\prime}\right) \rightarrow Z^{\prime}$ and $f=\pi \circ u$.

As a consequence, one has the following result.

Corollary 1.6.4. A surface $Z$ is relatively minimal if and only if $Z$ contains no (-1)-curve.

We are interested in a particular class of surfaces called rational.

Let $\mathbb{P}^{1}:=\mathbb{P}_{k}^{1}$ be the projective line over field $k$. A surface $Z$ is called birationally ruled if it is birationally equivalent to $C \times \mathbb{P}^{1}$ for some curve $C$. If $C=\mathbb{P}^{1}, Z$ is said to be a rational surface. A geometrically ruled surface, or simply a ruled surface, is a surface $Z$, together with a surjective projective morphism $\pi: Z \rightarrow C$ to a (nonsingular) curve $C$, such that the fibre $Z_{p}:=\pi^{-1}(p)$ is isomorphic to $\mathbb{P}^{1}$ for every point $p \in C$, and such that $\pi$ admits a section (i.e., a morphism $\sigma: C \rightarrow X$ such that $\left.\pi \circ \sigma=\operatorname{Id}_{C}\right)$. It holds that every ruled surface is birationally ruled [71, Chapter V, Proposition 2.2].

An example of rational surface is the projective plane $\mathbb{P}^{2}:=\mathbb{P}_{k}^{2}$ over $k$. Other examples are the surfaces we next define.

Definition 1.6.5. Let $\delta$ be a non-negative integer. The $\delta$-th Hirzebruch surface is the projective space $\mathbb{F}_{\delta}:=\mathbb{P}\left(\mathcal{O}_{\mathbb{P}^{1}} \oplus \mathcal{O}_{\mathbb{P}^{1}}(-\delta)\right)$.

The Hirzebruch surfaces are ruled surfaces over $\mathbb{P}^{1}$ and every ruled surface $Z$ over $\mathbb{P}^{1}$ is isomorphic to $\mathbb{P}\left(\mathcal{O}_{\mathbb{P}^{1}} \oplus \mathcal{O}_{\mathbb{P}^{1}}(-\delta)\right)$ for some non-negative integer $\delta$ [9, Chapter III, Proposition III.15]. As a result, the Hirzebruch surfaces are rational surfaces. In addition, we have the following consequence of [9, Chapter V, Theorem V.10] and Corollary 1.6.4.

Theorem 1.6.6. $Z$ is a relatively minimal rational surface if and only if $Z$ is isomorphic to $\mathbb{P}^{2}$ or to one of the surfaces $\mathbb{F}_{\delta}$, for $\delta=0$ or $\delta \geq 2$.

The above result allows us to conclude that every rational surface can be seen as a surface created by a finite sequence of point blowups over $\mathbb{P}^{2}$ or $\mathbb{F}_{\delta}$, where $\delta$ is a non-negative integer different from 1.

To finish this first part of this section, we notice that two divisors on $\mathbb{P}^{2}$ or $\mathbb{F}_{\delta}$ are linearly equivalent if and only if they are numerically equivalent. As a result, it holds that

$$
\operatorname{Pic}\left(\mathbb{P}^{2}\right)=\operatorname{Num}\left(\mathbb{P}^{2}\right) \text { and } \operatorname{Pic}\left(\mathbb{F}_{\delta}\right)=\operatorname{Num}\left(\mathbb{F}_{\delta}\right)
$$

\subsubsection{Hirzebruch surfaces}

We devote this subsection to give an extended description of the Hirzebruch surfaces. Recall that $k$ is an algebraically closed field of arbitrary characteristic. 
Let $\mathbb{F}_{\delta}$ be a Hirzebruch surface over $k$ and $p r: \mathbb{F}_{\delta} \rightarrow \mathbb{P}^{1}$ the projective morphism associated to $\mathbb{F}_{\delta}$ (since $\mathbb{F}_{\delta}$ is a ruled surface). Denote by $\operatorname{Pic}\left(\mathbb{F}_{\delta}\right)$ the Picard group of $\mathbb{F}_{\delta}$ and by $[D]$ the linear equivalence class of a divisor $D$. It holds that $\operatorname{Pic}\left(\mathbb{F}_{\delta}\right)$ is isomorphic to $\mathbb{Z} \oplus \mathbb{Z}$ and it is generated by the set $\{[F],[M]\}$, where $F$ is a fiber of $p r$ and $M$ a section of $p r$ whose self-intersection is $\delta$. The matrix of the bilinear pairing of $\operatorname{Pic}\left(\mathbb{F}_{\delta}\right)$ with respect to that basis is

$$
\left(\begin{array}{ll}
0 & 1 \\
1 & \delta
\end{array}\right) .
$$

When $\delta$ is positive, there exists a unique irreducible curve on $\mathbb{F}_{\delta}$ with negative selfintersection. That curve is linearly equivalent to $-\delta F+M$ and its self-intersection equals $-\delta$ (see [9, Chapter IV, Proposition IV.1]); it is denoted by $M_{0}$ and is usually called the special section. Notice that, if $\delta=0, M_{0}$ will denote a section such that $M_{0}^{2}=0$.

The following result will be useful.

Proposition 1.6.7. Let $\mathbb{F}_{\delta}$ be a Hirzebruch surface.

(a) Let $C$ be an irreducible curve $C \neq F, M_{0}$. Then $C \sim a F+b M$, where $a \geq 0$ and $b>0$.

(b) The cone of curves $\mathrm{NE}\left(\mathbb{F}_{\delta}\right)$ is generated by the class of a fiber $F$ and that of the section $M_{0}$.

(c) A divisor $D \sim a F+b M$ on $\mathbb{F}_{\delta}$ is nef if and only if $a \geq 0$ and $b \geq 0$.

(d) A divisor $D \sim a F+b M$ on $\mathbb{F}_{\delta}$ is big if and only if $b>0$ and $a>-\delta b$.

Proof. Item (a) follows from [71, Chapter V, Proposition 2.20].

Item (b) holds by (a) and by the fact that $M_{0}$ is the curve with non-positive self-intersection on $\mathbb{F}_{\delta}$.

Now we are going to prove (c). By (b), one has that a divisor $D \sim a F+b M$ is nef if and only if $D \cdot F \geq 0$ and $D \cdot M_{0} \geq 0$. Then, a divisor $D$ is nef if and only if $b \geq 0$ and $a \geq 0$.

Finally we show (d). A divisor $D \sim a F+b M$ is big if and only if $D \cdot D^{\prime}>0$ for every nef divisor $D^{\prime}$ on $\mathbb{F}_{\delta}$. Taking into account (c), one obtains that the nef divisors are generated by the class of a fiber $F$ and that of a section $M$. As a consequence, a divisor $D$ is big if and only if the conditions $b=D \cdot F>0$ and $a+b \delta=D \cdot M>0$ hold.

Remark 1.6.8. Let $\mathbb{F}_{\delta}$ be a Hirzebruch surface. When $\delta$ is a positive integer, we can find big divisors $D$ on $\mathbb{F}_{\delta}$ which are not nef. In fact, these divisors are linearly equivalent to $a F+b M$, where $b \in \mathbb{Z}_{>0}$ and $-\delta b<a<0$. In addition, it is easy to check that the Zariski decomposition of $D, D=P_{D}+N_{D}$, is $P_{D} \sim(b+a / \delta) M$ and $N_{D} \sim(-a / \delta) M_{0}$. 
Let $\mathbb{A}^{2}=\mathbb{A}_{k}^{2}$ be the affine plane over $k$. A Hirzebruch surface $\mathbb{F}_{\delta}$ can be obtained as the quotient of $\left(\mathbb{A}^{2} \backslash\{(0,0)\}\right) \times\left(\mathbb{A}^{2} \backslash\{(0,0)\}\right)$ by an action of the product of multiplicative groups, $k^{*} \times k^{*}$, where $k^{*}=k \backslash\{0\}$ (see [102, Chapter 2]). Let $X_{0}, X_{1}$ be coordinates in the first factor and $Y_{0}, Y_{1}$ coordinates in the second one. For each $(\lambda, \mu) \in k^{*} \times k^{*}$ the action is defined as follows:

$$
\begin{gathered}
(\lambda, 1):\left(X_{0}, X_{1} ; Y_{0}, Y_{1}\right) \rightarrow\left(\lambda X_{0}, \lambda X_{1} ; Y_{0}, \lambda^{-\delta} Y_{1}\right) \\
(1, \mu):\left(X_{0}, X_{1} ; Y_{0}, Y_{1}\right) \rightarrow\left(X_{0}, X_{1} ; \mu Y_{0}, \mu Y_{1}\right)
\end{gathered} .
$$

Note that the action preserves the ratio $\left(X_{0}: X_{1}\right)$ and then the projective morphism $p r: \mathbb{F}_{\delta} \rightarrow \mathbb{P}^{1}$ is just the projection onto the first factor.

On account of [30] and [90, Section 1.2], the homogeneous coordinate ring of $\mathbb{F}_{\delta}$ is $S_{\delta}:=k\left[X_{0}, X_{1}, Y_{0}, Y_{1}\right]$, where each variable is graded on $\mathbb{Z} \times \mathbb{Z} \geq 0$ as follows

$$
\operatorname{deg} X_{0}=(1,0), \operatorname{deg} X_{1}=(1,0), \operatorname{deg} Y_{0}=(0,1), \operatorname{deg} Y_{1}=(-\delta, 1) .
$$

The set of homogeneous elements of degree $(a, b) \in \mathbb{Z} \times \mathbb{Z}_{\geq 0}$ in $S_{\delta}$ is described as

$$
S_{\delta}(a, b):=\bigoplus_{\alpha_{0}+\alpha_{1}=\delta \beta_{1}+a, \beta_{0}+\beta_{1}=b} k X_{0}^{\alpha_{0}} X_{1}^{\alpha_{1}} Y_{0}^{\beta_{0}} Y_{1}^{\beta_{1}}
$$

and so, it is said that an integral curve $C$ on $\mathbb{F}_{\delta}$ has degree $(a, b)$ if it is defined by an irreducible and reduced polynomial $H \in S_{\delta}(a, b)$. Recall that, by Proposition 1.6.7(a), any irreducible curve $C$ of degree $(a, b)$, different from a fiber $F$ and $M_{0}$, is linearly equivalent to $a F+b M$, where $a \geq 0$ and $b>0$.

Assume that $\delta$ is positive. The above development shows that an irreducible curve on $\mathbb{F}_{\delta}$ of degree $(1,0)$ is linearly equivalent to a fiber $F$ and it is defined by the equation $a X_{0}+b X_{1}=0$, where $a, b \in k$ and at least one of them is not equal to zero. Similarly, an irreducible curve of degree $(0,1)$ on $\mathbb{F}_{\delta}$ is linearly equivalent to $M$ and its equation is $a Y_{0}+\sum_{i=0}^{\delta} b_{i} X_{0}^{i} X_{1}^{\delta-i} Y_{1}=0$, where $a \in k^{*}$ and $b_{i} \in k$. Finally, one can see that $M_{0}$ is defined by the equation $Y_{1}=0$.

Notice that a point $p \in \mathbb{F}_{\delta}$ determines a fiber $F$ and $p$ cannot belong to the intersection of the special section $M_{0}$ and an irreducible curve linearly equivalent to $M$. Next, we distinguish two types of points in $\mathbb{F}_{\delta}$ which will be considered along this work. A point $p \in \mathbb{F}_{\delta}$ is called a special point if $p$ belongs to the special section. Otherwise, $p$ is named a general point.

A useful result about the geometry of a Hirzebruch surface $\mathbb{F}_{\delta}$, where $\delta \geq 1$, is the following one stated in [90].

Proposition 1.6.9. Assume that $\delta \geq 1$. Then, through any general point of $\mathbb{F}_{\delta}$ a $\delta$ dimensional family of irreducible curves linearly equivalent to $M$ goes. Furthermore, an irreducible curve of degree $(1,0)$ and an irreducible curve of degree $(0,1)$ meet at a general point. 
Proof. Let $\left(p_{x_{0}}: p_{x_{1}} ; p_{y_{0}}, p_{y_{1}}\right)$ be the coordinates of a general point $p \in \mathbb{F}_{\delta}$; this means $p_{y_{1}} \neq 0$. In addition $p$ determines a fiber $F$ and so either $p_{x_{0}}$ or $p_{x_{1}}$ does not vanish. Consequently, with the previous notation, one obtains that

$$
b_{\delta}=\frac{a p_{y_{0}}+\sum_{i=0}^{\delta-1} b_{i}\left(p_{x_{0}}\right)^{i}\left(p_{x_{1}}\right)^{\delta-i} p_{y_{1}}}{\left(p_{x_{0}}\right)^{\delta} p_{y_{1}}} \text { or } b_{0}=\frac{a p_{y_{0}}+\sum_{i=1}^{\delta} b_{i}\left(p_{x_{0}}\right)^{i}\left(p_{x_{1}}\right)^{\delta-i} p_{y_{1}}}{\left(p_{x_{1}}\right)^{\delta} p_{y_{1}}}
$$

which finishes the proof of our first statement taking into account $a \in k^{*}$.

The second one holds since an irreducible curve of degree $(1,0)$ (respectively, an irreducible curve of degree $(0,1))$ is linearly equivalent to $F$ (respectively, $M$ ) and $F \cdot M=1$.

When $\delta=0$, the Hirzebruch surface is $\mathbb{F}_{0}=\mathbb{P}\left(\mathcal{O}_{\mathbb{P}^{1}} \oplus \mathcal{O}_{\mathbb{P}^{1}}\right)$ and this surface is isomorphic to $\mathbb{P}^{1} \times \mathbb{P}^{1}$ by (1.13). This gives rise to two morphisms to $\mathbb{P}^{1}$, obtaining a double ruling structure. Note that, in this case, there is no special section $\left(M_{0} \sim M\right)$ and the matrix of the bilinear pairing is

$$
\left(\begin{array}{ll}
0 & 1 \\
1 & 0
\end{array}\right)
$$

The homogeneous coordinate ring of $\mathbb{F}_{0}$ is $S_{0}:=k\left[X_{0}, X_{1}, Y_{0}, Y_{1}\right]$ whose variables have the following degrees:

$$
\operatorname{deg} X_{0}=(1,0)=\operatorname{deg} X_{1} \quad \text { and } \quad \operatorname{deg} Y_{0}=(0,1)=\operatorname{deg} Y_{1} .
$$

The homogeneous part of degree $(a, b) \in \mathbb{Z}_{\geq 0} \times \mathbb{Z}_{\geq 0}$ of $S_{0}$ equals

$$
S_{0}(a, b)=\bigoplus_{\alpha_{0}+\alpha_{1}=a, \beta_{0}+\beta_{1}=b} k X_{0}^{\alpha_{0}} X_{1}^{\alpha_{1}} Y_{0}^{\beta_{0}} Y_{1}^{\beta_{1}}
$$

and an irreducible curve $C$ of degree $(a, b)$ on $\mathbb{F}_{0}$ is linearly equivalent to $a F+$ $b M$, where $b>0$ and $a \geq 0$. In addition, when studying $\mathbb{F}_{0}$, there is no need of distinguishing between special and general points.

Proposition 1.6.10. [90, Lemma 1.2.3] A point $p$ in $\mathbb{F}_{0}$ determines and it is determined by a unique curve $F$ of degree $(1,0)$ and a unique curve $M$ of degree $(0,1)$.

Notice that a point $p=\left(p_{x_{0}}: p_{x_{1}} ; p_{y_{0}}, p_{y_{1}}\right)$ in $\mathbb{F}_{0}$ defines and is defined by an irreducible curve $F$ of degree $(1,0)$ with equation $p_{x_{1}} X_{0}-p_{x_{0}} X_{1}=0$ and an irreducible curve $M$ of degree $(0,1)$ with equation $p_{y_{1}} Y_{0}-p_{y_{0}} Y_{1}=0$.

Remark 1.6.11. When considering either a point $p \in \mathbb{F}_{0}$ or a special point in $\mathbb{F}_{\delta}$, $\delta \geq 1$, we get the same behaviour as in Proposition 1.6.10: a point in $\mathbb{F}_{0}$ determines a unique fiber and a unique section $M$, and a special point belongs to the special section and it determines a unique fiber $F$. 
To finish this section, we show some details about the local structure of a Hirzebruch surface.

Let $\delta$ be a non-negative integer and $\mathbb{F}_{\delta}$ a Hirzebruch surface. $\mathbb{F}_{\delta}$ is covered by four open sets, all of them isomorphic to $\mathbb{A}^{2}$. These open sets will be denoted by $U_{i, j}:=\mathbb{F}_{\delta} \backslash \mathbf{V}\left(X_{i} Y_{j}\right)$, for $0 \leq i, j \leq 1$, where $\mathbf{V}\left(X_{i} Y_{j}\right)$ is the closed set in $\mathbb{F}_{\delta}$ described by the points which satisfy $X_{i} Y_{j}=0$.

Indeed, when $i=j=0$, taking $\lambda=1 / X_{0}$, it holds that

$$
\left(X_{0}: X_{1} ; Y_{0}, Y_{1}\right) \equiv\left(1: X_{1} / X_{0} ; Y_{0}, X_{0}^{\delta} Y_{1}\right)
$$

Now, if we pick $\mu=1 / Y_{0}$, one obtains that

$$
\left(1: X_{1} / X_{0} ; Y_{0}, X_{0}^{\delta} Y_{1}\right) \equiv\left(1: X_{1} / X_{0} ; 1,\left(X_{0}^{\delta} Y_{1}\right) / Y_{0}\right)
$$

As $X_{0}$ and $Y_{0}$ are not equal to zero, and $X_{1}$ and $Y_{1}$ can take any value in $k$, we get affine coordinates $\left(1: X_{1} / X_{0} ; 1,\left(X_{0}^{\delta} Y_{1}\right) / Y_{0}\right) \cong(u, v)$, where $(u, v) \in \mathbb{A}^{2}$. Analogous descriptions can be done for the remaining open sets.

The following result provides some changes of coordinates for the previous open sets.

Proposition 1.6.12. Let $U_{i, j}$ be the open sets of the surface $\mathbb{F}_{\delta}$ above defined. Consider, as described, affine coordinates $(u, v)$ for $U_{i, j}$ and $\left(u^{\prime}, v^{\prime}\right)$ for $U_{k, l}$, where $i, j, k, l \in\{0,1\}$. Then

(a)

$$
\left\{\begin{array}{l}
u^{\prime}=\frac{1}{u} \\
v^{\prime}=\frac{1}{u^{\delta} v}
\end{array} \text {, if } i=j=0 \text { and } k=l=1\right. \text {. And }
$$

(b)

$$
\left\{\begin{array}{l}
u^{\prime}=\frac{1}{u} \\
v^{\prime}=\frac{v}{u^{\delta}}
\end{array}, \text { if } i=0 \text { and } j=k=l=1 .\right.
$$

Proof. We prove (a). A proof for (b) runs similarly. Consider a point $p \in U_{0,0} \cap U_{1,1}$. Then the coordinates of $p$ in $U_{0,0}$ are $\left(p_{x_{0}}: p_{x_{1}} ; p_{y_{0}}, p_{y_{1}}\right)$, where $p_{x_{0}} \neq 0$ and $p_{y_{0}} \neq 0$, and

$$
\left(p_{x_{0}}: p_{x_{1}} ; p_{y_{0}}, p_{y_{1}}\right) \equiv\left(1: \frac{p_{x_{1}}}{p_{x_{0}}} ; 1, \frac{\left(p_{x_{0}}\right)^{\delta} p_{y_{1}}}{p_{y_{0}}}\right)
$$

Moreover, $p \in U_{1,1}$, therefore

$$
\left(p_{x_{0}}: p_{x_{1}} ; p_{y_{0}}, p_{y_{1}}\right) \equiv\left(\frac{p_{x_{0}}}{p_{x_{1}}}: 1 ; \frac{p_{y_{0}}}{\left(p_{x_{1}}\right)^{\delta} p_{y_{1}}}, 1\right)
$$


Denote $\frac{p_{x_{1}}}{p_{x_{0}}}$ and $\frac{\left(p_{x_{0}}\right)^{\delta} p_{y_{1}}}{p_{y_{0}}}$ by $u$ and $v$, respectively; and $\frac{p_{x_{0}}}{p_{x_{1}}}$ and $\frac{p_{y_{0}}}{\left(p_{x_{1}}\right)^{\delta} p_{y_{1}}}$ by $u^{\prime}$ and $v^{\prime}$, respectively. As a consequence, it holds that

$$
\begin{gathered}
u^{\prime}=\frac{p_{x_{0}}}{p_{x_{1}}}=\frac{1}{u}, \text { and } \\
v^{\prime}=\frac{p_{y_{0}}}{\left(p_{x_{1}}\right)^{\delta} p_{y_{1}}}=\frac{p_{y_{0}}}{\left(p_{x_{0}}\right)^{\delta} p_{y_{1}}} \frac{\left(p_{x_{0}}\right)^{\delta}}{\left(p_{x_{1}}\right)^{\delta}}=\frac{1}{u^{\delta} v},
\end{gathered}
$$

which finishes the proof. 


\section{Chapter 2}

\section{Non-positive and negative at infinity valuations of a rational surface}

In this chapter we introduce the concepts of non-positivity and negativity at infinity of divisorial and irrational valuations of the projective plane $\mathbb{P}^{2}$ and of a Hirzebruch surface $\mathbb{F}_{\delta}, \delta \geq 0$. We will show that rational surfaces defined by divisorial valuations of the above types enjoy good geometrical properties. Asymptotic results could be deduced from real valuations. For our study, we divide the above considered valuations into three types. Since these valuations are essentially characterized by their dual graphs (as we will explain), we study their dual graphs and provide an algorithm to generate them. Our main results and proofs concerning these objects can be found in [63] and [61].

To start we introduce the notation that we use in this chapter.

Let $k$ be an arbitrary algebraically closed field. Assume that the surface $Z_{0}$ is either the projective plane $\mathbb{P}^{2}$ or a Hirzebruch surface $\mathbb{F}_{\delta}, \delta \geq 0$, over $k$. Consider a finite or infinite simple sequence of blowups

$$
\pi: \cdots \rightarrow Z_{n} \stackrel{\pi_{n}}{\longrightarrow} Z_{n-1} \rightarrow \cdots \rightarrow Z_{1} \stackrel{\pi_{1}}{\longrightarrow} Z_{0}
$$

where each blowup $\pi_{i}: Z_{i} \rightarrow Z_{i-1}$ is centered at a closed point $p_{i} \in Z_{i-1}$ such that $p_{1}=p \in Z_{0}$ and, otherwise, $p_{i}$ belongs to the exceptional divisor created by $\pi_{i-1}$. Abusing of notation, for each surface $Z_{n}$ (with $n \geq 1$ ), we denote by $E_{i}$ (respectively, $\left.E_{i}^{*}\right), i \leq n$, the strict (respectively, total) transform on $Z_{n}$ of the exceptional divisor $E_{i}$ created after blowing-up $p_{i}$ and by $\tilde{D}$ (respectively, $D^{*}$ ) the strict (respectively, total) transform on $Z_{n}$ of a divisor $D$ on $Z_{i}$. Write $\varphi_{C}$ (respectively, $\varphi_{i}$ ) the germ of a curve $C$ at $p$ (respectively, an analytically irreducible germ at $p$ whose strict transform on $Z_{i}$ is transversal to $E_{i}$ at a non-singular point of the exceptional locus).

In this chapter we only consider divisorial and irrational valuations. Set $\nu$ the 
divisorial or irrational valuation defined by a simple sequence of blowups as (2.1). Frequently and for short, $\nu$ is usually called a divisorial or irrational valuation of $Z_{0}$. Recall that if $\pi$ is finite, then $\nu$ is a divisorial valuation and this valuation is defined by the last exceptional divisor $E_{n}$. Often, when the situation requires it, the valuation $\nu$ is denoted $\nu_{n} \cdot \mathcal{C}_{\nu}=\left\{p_{i}\right\}_{i \geq 1}$ stands for the configuration of infinitely near points of $\nu$, the dual graph of $\nu$ is denoted by $\Gamma_{\nu}$ and its corresponding subgraphs by $\Gamma_{\nu}^{i}$, for $i \in\{1,2, \ldots, g+1\}$. We also denote by $\left\{\bar{\beta}_{i}(\nu)\right\}_{i=0}^{g+1}$ (respectively, $\left.\left\{\beta_{i}^{\prime}(\nu)\right\}_{i=0}^{g+1}\right)$ the sequence of maximal contact values (respectively, the sequence of Puiseux exponents) associated to $\nu$.

Let $\nu_{n}$ be the divisorial valuation of the function field of $Z_{0}$ centered at $\mathcal{O}_{Z_{0}, p}$ defined by the exceptional divisor $E_{n}$. Set $Z:=Z_{n}$ the surface generated by $\nu_{n}$. According the previous chapter, it holds that

$$
\operatorname{Pic}(Z) \cong \operatorname{Pic}\left(Z_{0}\right) \oplus \mathbb{Z} E_{1}^{*} \oplus \mathbb{Z} E_{2}^{*} \oplus \cdots \oplus \mathbb{Z} E_{n}^{*}
$$

The corresponding bilinear paring is denoted by $\cdot$. Write $\operatorname{Pic}_{\mathbb{R}}(Z)$ the tensor product $\operatorname{Pic}(Z) \otimes \mathbb{R}$ and, by abuse of notation, $\cdot$ its bilinear pairing. Notice that $\operatorname{Pic}_{\mathbb{R}}(Z)=$ $\operatorname{Num}_{\mathbb{R}}(Z)$.

\subsection{Non-positive at infinity valuations of the projective plane}

In this section we assume that $Z_{0}=\mathbb{P}^{2}$. Let $\nu$ be a divisorial or irrational valuation of the function field of $\mathbb{P}^{2}$ centered at $\mathcal{O}_{\mathbb{P}^{2}, p}$ and $\pi$ the simple sequence of point blowups that it defines. We always suppose that the number of blowups is at least 2. Set $L$ the projective line (called the line at infinity) containing the point $p$ and whose strict transform passes through $p_{2}$.

Definition 2.1.1. Under the above assumptions, the valuation $\nu$ is called to be non-positive at infinity (NPI) when $\nu(f) \leq 0$, for all $f \in \mathcal{O}_{\mathbb{P}^{2}}\left(\mathbb{P}^{2} \backslash L\right)$. If $\nu(f)<0$ holds, for all $f \in \mathcal{O}_{\mathbb{P}^{2}}\left(\mathbb{P}^{2} \backslash L\right) \backslash k$, then $\nu$ is said to be negative at infinity (NI).

From a geometric point of view, NPI and NI divisorial valuations of $\mathbb{P}^{2}$ are interesting since they give us information about the cone of curves of the surfaces that they define, as we will see in the following results. These theorems can be found in [60] and our future Theorems 2.3.7 and 2.3.14 will extend them to the case $Z_{0}=\mathbb{F}_{\delta}$.

Theorem 2.1.2. Let $\nu_{n}$ be a divisorial valuation of $\mathbb{P}^{2}$ and $L$ the line at infinity. Set $\bar{\beta}_{g+1}\left(\nu_{n}\right)$ (respectively, $\left.\left\{\nu_{n}\left(\mathfrak{m}_{i}\right)\right\}_{i=1}^{n}\right)$ the last maximal contact value (respectively, the sequence of values) of $\nu_{n}$. Then, the following conditions are equivalent:

(a) The valuation $\nu_{n}$ is non-positive at infinity.

(b) $\nu_{n}\left(\varphi_{L}\right)^{2} \geq \bar{\beta}_{g+1}\left(\nu_{n}\right)$. 
(c) The divisor $\nu_{n}\left(\varphi_{L}\right) E_{0}^{*}-\sum_{i=1}^{n} \nu_{n}\left(\mathfrak{m}_{i}\right) E_{i}^{*}$, where $E_{0}^{*}$ is the total transform of a projective line $E_{0}$ which does not pass through $p$, is nef.

(d) The cone of curves $\operatorname{NE}(Z)$ is generated by the classes of $\tilde{L}, E_{1}, E_{2}, \ldots, E_{n}$ (i.e., it is regular).

Theorem 2.1.3. Keeping the assumptions and notation of Theorem 2.1.2, the following conditions are equivalent:

(a) The valuation $\nu_{n}$ is negative at infinity.

(b) Either $\nu_{n}\left(\varphi_{L}\right)^{2}>\bar{\beta}_{g+1}\left(\nu_{n}\right)$, or $\nu_{n}\left(\varphi_{L}\right)^{2}=\bar{\beta}_{g+1}\left(\nu_{n}\right)$ and the Iitaka dimension of $\nu_{n}\left(\varphi_{L}\right) E_{0}^{*}+\sum_{i=1}^{n} \nu_{n}\left(\mathfrak{m}_{i}\right) E_{i}^{*}$ vanishes.

(c) The intersection product of $\nu_{n}\left(\varphi_{L}\right) E_{0}^{*}+\sum_{i=1}^{n} \nu_{n}\left(\mathfrak{m}_{i}\right) E_{i}^{*}$ and the strict transform of any integral curve $C$ on $\mathbb{P}^{2}$ different from the projective line $L$ is positive.

\subsection{Valuations of a Hirzebruch surface}

This section considers divisorial and irrational valuations of a Hirzebruch surface and divides them into two types named special and non-special.

In this section we assume that the surface $Z_{0}$ of the sequence $\pi(2.1)$ is a Hirzebruch surface $\mathbb{F}_{\delta}, \delta \geq 0$, and $p$ a closed point in $\mathbb{F}_{\delta}$. Recall that a basic introduction about Hirzebruch surfaces was provided in Subsection 1.6.1. Let $\nu_{n}$ be a divisorial valuation of the function field of $\mathbb{F}_{\delta}$ centered at $\mathcal{O}_{\mathbb{F}_{\delta}, p}$.

Definition 2.2.1. A divisorial valuation $\nu_{n}$ as above is called to be special (with respect to $\mathbb{F}_{\delta}$ and $p$ ) when one of the following conditions holds:

1. $\delta=0$.

2. $\delta>0$ and $p$ is a special point.

3. $\delta>0, p$ is a general point and there is no integral curve in the complete linear system $|M|$ whose strict transform on $Z$ has negative self-intersection.

Otherwise, $\nu_{n}$ will be named non-special.

We start with a property of non-special divisorial valuations.

Proposition 2.2.2. Let $\nu_{n}$ be a non-special divisorial valuation and $\mathcal{C}_{\nu_{n}}=\left\{p_{i}\right\}_{i=1}^{n}$ its configuration of infinitely near points. Suppose also that there exists an integral curve $M^{\prime}$, linearly equivalent to $M$, going through $p=p_{1}$ and whose strict transform passes through $p_{2}$. Then, there exists a unique integral curve $M_{1} \in|M|$ such that $M_{1}$ goes through $p$ and their strict transforms pass through the points $p_{2}, p_{3}, \ldots, p_{\delta+1}$. 
Proof. We start by showing the existence of $M_{1}$. Without loss of generality suppose that the general point $p$ has coordinates $(0,1 ; 0,1) \in U_{11}$. Pick affine coordinates $\{u, v\}=\left\{\frac{X_{0}}{X_{1}}, \frac{Y_{0}}{X_{1}^{\delta} Y_{1}}\right\}$ for which $p=(0,0)$. By Subsection 1.6.1, an integral curve of degree $(0,1)$ passing through $p$ is defined by the equation $a Y_{0}+\sum_{i=1}^{\delta} b_{i} X_{0}^{i} X_{1}^{\delta-i} Y_{1}=0$, where $a \in k^{*}$ and $b_{i} \in k, 1 \leq i \leq \delta$. One can assume $a=1$ with local equation (in $\left.U_{11}\right) v+\sum_{i=1}^{\delta} b_{i} u^{i}=0$.

Blowing up the point $p$ and looking at the strict transform of curves as $M$, we notice the existence of a $(\delta-1)$-dimensional family of curves linearly equivalent to $\tilde{M}=M^{*}-E_{1}^{*}$ which goes through each point of the exceptional divisor $E_{1}$ with the exception of the point at infinity. If now we blow any point different from the infinity, we obtain a similar situation appearing a $(\delta-2)$-dimensional family of curves linearly equivalent to $\tilde{M}=M^{*}-E_{1}^{*}-E_{2}^{*}$ going through each free point of $E_{2}$. Repeating this procedure and noticing that every point $p_{i}, i \leq \delta+1$, is free, we conclude the existence of a section $M_{1}$ as in the statement.

To finish let us show the uniqueness. Suppose that the strict transforms $\tilde{M}_{1}$ and $\tilde{M}_{2}$ of two curves $M_{1}$ and $M_{2}$ of degree $(0,1)$ go through $p_{\delta+1}$. Then $\tilde{M}_{1}$ and $\tilde{M}_{2}$ also pass through $p_{i}, 1 \leq i \leq \delta$. This fact fixes the values $b_{i}$ in the above given equation for $M_{1}$ and $M_{2}$. As a consequence, the equations of $M_{1}$ and $M_{2}$ are the same and we conclude the proof.

Remark 2.2.3. By the above proposition, another way of stating the condition (3) of Definition 2.2.1 is to say: $\delta>0, p$ is a general point and, either $p_{2} \in \mathcal{C}_{\nu_{n}}$ belongs to strict transform of the fiber of $p r$ passing through $p$ on $Z_{1}$, or there does not exist any $j \geq \delta+1$ such that the points $p_{i}, 1 \leq i \leq j$, of $\mathcal{C}_{\nu_{n}}$ are free.

Let us see how our types of divisorial valuations can be extended to irrational ones.

Definition 2.2.4. Let $\nu$ be an irrational valuation of $\mathbb{F}_{\delta}$. Set $\nu_{i}^{N}$ the normalized divisorial valuations of $\mathbb{F}_{\delta}$ which satisfy $\nu(f)=\lim _{i \rightarrow \infty} \nu_{i}^{N}(f)$, for all $f \in \mathcal{O}_{\mathbb{F}_{\delta}, p}$. Then $\nu$ is said special (respectively, non-special) when so are $\nu_{i}^{N}, i \gg 1$.

Let $\nu$ be a divisorial or irrational valuation of $\mathbb{F}_{\delta}$ centered at $\mathcal{O}_{\mathbb{F}_{\delta}, p}$. Denote by $F_{1}$ the fiber of $p r$ which contains the point $p$. When $\nu$ is non-special, $M_{1}$ is the curve provided by Proposition 2.2.2 whose strict transform has negative self-intersection on the surface defined by $\nu$ (if $\nu$ is divisorial), or by $\nu_{i}^{N}, i \gg 1$ (otherwise).

Definition 2.2.5. Under the above notation, a special (respectively, non-special) divisorial or irrational valuation $\nu$ of $\mathbb{F}_{\delta}$ is called non-positive at infinity (NPI) when $\nu(f) \leq 0$, for $f \in \mathcal{O}_{\mathbb{F}_{\delta}}\left(\mathbb{F}_{\delta} \backslash\left(F_{1} \cup M_{0}\right)\right.$ ) (respectively, $f \in \mathcal{O}_{\mathbb{F}_{\delta}}\left(\mathbb{F}_{\delta} \backslash\left(F_{1} \cup M_{1}\right)\right)$ ). And, $\nu$ is said to be negative at infinity (NI) if $\nu(f)<0$, for $f \in \mathcal{O}_{\mathbb{F}_{\delta}}\left(\mathbb{F}_{\delta} \backslash\left(F_{1} \cup M_{0}\right)\right)$, $f \notin k$ (respectively, $\left.f \in \mathcal{O}_{\mathbb{F}_{\delta}}\left(\mathbb{F}_{\delta} \backslash\left(F_{1} \cup M_{1}\right)\right), f \notin k\right)$. 


\subsection{The cone of curves of a surface defined by an NPI special divisorial valuation of a Hirzebruch surface}

Our goal in this section is to give a geometric characterization to the fact that a special divisorial valuation of a Hirzebruch surface $\mathbb{F}_{\delta}, \delta \geq 0$, is non-positive, or negative, at infinity.

Let $p$ be a point of a Hirzebruch surface $\mathbb{F}_{\delta}, \delta \geq 0$, and $\nu_{n}$ a special divisorial valuation of the function field of $\mathbb{F}_{\delta}$ centered at $\mathcal{O}_{\mathbb{F}_{\delta}, p}$. Keeping the notation of the above sections, denote by $F_{1}$ the fiber of $p r$ going through the point $p$ and $M_{0}$ the special section, or the section of $p r$ containing $p$ when $\delta=0$. Recall that $Z:=Z_{n}$ is the rational surface defined by the finite simple sequence of blowups

$$
\pi: Z:=Z_{n} \stackrel{\pi_{n}}{\longrightarrow} Z_{n-1} \rightarrow \cdots \rightarrow Z_{1} \stackrel{\pi_{1}}{\longrightarrow} Z_{0}=\mathbb{F}_{\delta}
$$

corresponding to the divisorial valuation $\nu_{n}$.

Notice that $\tilde{F}_{1}$ and $\tilde{M}_{0}$ have negative self-intersection on $Z$ since $F_{1}^{2}=0$ and $M_{0}^{2}=-\delta$. Therefore, by Proposition 1.4.6, the classes of $\tilde{F}_{1}$ and $\tilde{M}_{0}$ generate extremal rays of the cones $\mathrm{NE}(Z)$ and $\overline{\mathrm{NE}}(Z)$. For our purposes, we need to consider the strongly convex cone $S_{1}(Z)$ of $\operatorname{Pic}_{\mathbb{R}}(Z)$ generated by the set $\left\{\left[\tilde{F}_{1}\right],\left[\tilde{M}_{0}\right]\right\} \cup\left\{\left[E_{i}\right]\right\}_{i=1}^{n}$ and also its dual cone

$$
S_{1}^{\vee}(Z)=\left\{[D] \in \operatorname{Pic}_{\mathbb{R}}(Z) \mid[D] \cdot[C] \geq 0, \text { for all }[C] \in S_{1}(Z)\right\} .
$$

The following proposition provides generators for the cone $S_{1}^{\vee}(Z)$.

Proposition 2.3.1. The dual cone $S_{1}^{\vee}(Z)$ is generated by $\left[F^{*}\right],\left[M^{*}\right]$ and the classes $\left\{\left[\Lambda_{i}\right]\right\}_{i=1}^{n}$ of the divisors

$$
\Lambda_{i}:=a_{i} F^{*}+b_{i} M^{*}-\sum_{j=1}^{i} \operatorname{mult}_{p_{j}}\left(\varphi_{i}\right) E_{j}^{*},
$$

where $a_{i}:=\left(\varphi_{i}, \varphi_{M_{0}}\right)_{p}$ and $b_{i}:=\left(\varphi_{i}, \varphi_{F_{1}}\right)_{p}$.

Proof. By Proposition 1.4.2, it suffices to show that $\left\{\left[F^{*}\right],\left[M^{*}\right]\right\} \cup\left\{\left[\Lambda_{i}\right]\right\}_{i=1}^{n}$ is the dual basis of the basis $\left\{\left[\tilde{F}_{1}\right],\left[\tilde{M}_{0}\right]\right\} \cup\left\{\left[E_{i}\right]\right\}_{i=1}^{n}$ of $\operatorname{Pic}(Z)$ with respect to the intersection product.

Let $p_{i_{F_{1}}}$ be the last point in the configuration $\mathcal{C}_{\nu_{n}}$ of the valuation $\nu_{n}$ giving rise to $Z$ through which the strict transform of $F_{1}$ passes. Also, if $p$ belongs to $M_{0}$, we define $i_{M_{0}}$ such that $p_{i_{M_{0}}}$ is the last point of $\mathcal{C}_{\nu}$ through which the strict transform of $M_{0}$ passes; otherwise we define $i_{M_{0}}:=0$. Taking into account that $\varphi_{i}$ is analytically irreducible, the proximity equalities (Equation (1.2)) show that $\Lambda_{i} \cdot E_{j}=\delta_{i j}$, where $\delta_{i j}$ denotes the Kronecker delta. Also, for each $i \in\{1,2, \ldots, n\}$, it holds

$$
\Lambda_{i} \cdot \tilde{F}_{1}=b_{i}-\sum_{j=1}^{\min \left\{i, i_{F_{1}}\right\}} \operatorname{mult}_{p_{j}}\left(\varphi_{i}\right)=0
$$


and

$$
\Lambda_{i} \cdot \tilde{M}_{0}=a_{i}-\sum_{j=1}^{\min \left\{i, i_{M_{0}}\right\}} \operatorname{mult}_{p_{j}}\left(\varphi_{i}\right)=0,
$$

where the summations with upper index equal to 0 are defined to be 0 . Finally notice that $F^{*} \cdot \tilde{F}_{1}=0, F^{*} \cdot \tilde{M}_{0}=1, M^{*} \cdot \tilde{F}_{1}=1, M^{*} \cdot \tilde{M}_{0}=0$ and $F^{*} \cdot E_{i}=M^{*} \cdot E_{i}=0$ for all $i=1,2, \ldots, n$. This concludes the proof.

Remark 2.3.2. By Subsection 1.3.1, the divisor $\Lambda_{i}$ defined in (2.3) can also be written as

$$
\Lambda_{i}=\nu_{i}\left(\varphi_{M_{0}}\right) F^{*}+\nu_{i}\left(\varphi_{F_{1}}\right) M^{*}-\sum_{j=1}^{i} \nu_{i}\left(\mathfrak{m}_{j}\right) E_{j}^{*}
$$

where $\nu_{i}, 1 \leq i \leq n$, is the divisorial valuation of $\mathbb{F}_{\delta}$ defined by the divisor $E_{i}$ created in the sequence (2.2) and $\left\{\nu_{i}\left(\mathfrak{m}_{j}\right)\right\}_{j=1}^{i}$ its sequence of values. In addition, the selfintersection of $\Lambda_{i}$ is

$$
\Lambda_{i}^{2}=2 a_{i} b_{i}+\delta b_{i}^{2}-\bar{\beta}_{g+1}\left(\nu_{i}\right)=2 \nu_{i}\left(\varphi_{M_{0}}\right) \nu_{i}\left(\varphi_{F_{1}}\right)+\delta \nu_{i}\left(\varphi_{F_{1}}\right)^{2}-\bar{\beta}_{g+1}\left(\nu_{i}\right),
$$

where $\bar{\beta}_{g+1}\left(\nu_{i}\right)$ is the last maximal contact value of $\nu_{i}$.

From now on the expression shown in the right hand of Equation (2.4) will be denoted $\Lambda\left(\nu_{i}\right)$.

The following two results show that a divisorial valuation of $\mathbb{P}^{2}$ determines a special divisorial valuation of the Hirzebruch surface $\mathbb{F}_{1}$ providing the same surface $Z$. These results also relate some useful divisors and invariants corresponding to both valuations.

Proposition 2.3.3. Let $\nu_{n}$ be a divisorial valuation of $\mathbb{P}^{2}$ and $L$ the line at infinity as in Subsection 2.1. Set $\mathcal{C}_{\nu_{n}}=\left\{p_{i}\right\}_{i=1}^{n}$ (respectively, $\left\{\nu_{n}\left(\mathfrak{m}_{i}\right)\right\}_{i=1}^{n}$ ) the configuration of infinitely near points (respectively, the sequence of values) of $\nu_{n}$ and consider $\nu_{n}\left(\varphi_{L}\right) E_{0}^{*}-\sum_{i=1}^{n} \nu_{n}\left(\mathfrak{m}_{i}\right) E_{i}^{*}$ the divisor defined in Theorem 2.1.2(c). Then there exists a special divisorial valuation $\nu$ of $\mathbb{F}_{1}$ defining the same surface $Z$ as $\nu_{n}$ such that

$$
E_{0}^{*}-E_{1}^{*} \sim F^{*} \text { and } \nu_{n}\left(\varphi_{L}\right) E_{0}^{*}-\sum_{i=1}^{n} \nu_{n}\left(\mathfrak{m}_{i}\right) E_{i}^{*} \sim \Lambda(\nu), \text { for } n>1 .
$$

Proof. Consider the finite sequence defined by $\nu_{n}$ (finishing at $Z=Z_{n}$ ) given in (2.2). For $1 \leq i \leq n$, denote by $E_{p_{i}}$ the exceptional divisor created after blowingup $p_{i}$. By [9, Chapter IV, Proposition IV.1], $\mathbb{F}_{1}$ can be regarded as the projective plane $\mathbb{P}^{2}$ with the point $p$ blown up. Since we are considering that $p=p_{1} \in L$ (see Subsection 2.1), any fiber $F$ of $p r$ is a curve which belongs to $|\tilde{L}|$, where $\tilde{L}$ is the strict transform of $L$ on $Z_{1}=\mathbb{F}_{1}$, and $M_{0} \sim E_{p_{1}}$ on $\mathbb{F}_{1}$. Now, take $\mathcal{C}_{\nu}=\left\{q_{i}\right\}_{i=1}^{n-1}$ such that $q_{i}=p_{i+1}$, for $1 \leq i \leq n-1$; it is the configuration of infinitely near points of a special divisorial valuation $\nu$ of $\mathbb{F}_{1}$ and defines the same surface $Z$ as $\nu_{n}$. Set $E_{q_{i}}$ the 
exceptional divisor created after blowing-up $q_{i}$, for $1 \leq i \leq n-1$. Write $\left\{\nu\left(\mathfrak{n}_{i}\right)\right\}_{i=1}^{n-1}$ the sequence of values of $\nu$. It holds that

$$
\nu\left(\varphi_{M_{0}}\right)=\nu_{n}\left(\mathfrak{m}_{1}\right) \text { and } \nu\left(\mathfrak{n}_{i}\right)=\nu_{n}\left(\mathfrak{m}_{i+1}\right), \text { for } 1 \leq i \leq n-1
$$

Suppose now that $p_{i_{L}}$ is the last point of $\mathcal{C}_{\nu_{n}}$ through which $\tilde{L}$ goes and set $F_{1}$ the fiber which contains $q_{1}=p_{2}$ on $\mathbb{F}_{1}$ whose strict transforms pass through $q_{2}, q_{3}, \ldots, q_{i_{F_{1}}}=$ $p_{i_{L}}$. Then

$$
\begin{aligned}
\nu_{n}\left(\varphi_{L}\right)=\sum_{j=1}^{i_{L}} \nu_{n}\left(\mathfrak{m}_{j}\right) \cdot \operatorname{mult}_{p_{j}}\left(\varphi_{L}\right) & =\sum_{j=1}^{i_{F_{1}}} \nu\left(\mathfrak{n}_{j}\right) \cdot \operatorname{mult}_{q_{j}}\left(\varphi_{F_{1}}\right)+\nu_{n}\left(\mathfrak{m}_{1}\right) \\
& =\nu\left(\varphi_{F_{1}}\right)+\nu\left(\varphi_{M_{0}}\right) .
\end{aligned}
$$

As a result, one has that

$$
\begin{aligned}
\Lambda(\nu) & =\nu\left(\varphi_{M_{0}}\right) F^{*}+\nu\left(\varphi_{F_{1}}\right) M^{*}-\sum_{i=1}^{n-1} \nu\left(\mathfrak{n}_{i}\right) E_{q_{i}}^{*} \\
& \sim\left(\nu\left(\varphi_{M_{0}}\right)+\nu\left(\varphi_{F_{1}}\right)\right) F^{*}+\nu\left(\varphi_{F_{1}}\right) M_{0}^{*}-\sum_{i=1}^{n-1} \nu\left(\mathfrak{n}_{i}\right) E_{q_{i}}^{*} \\
& \sim \nu_{n}\left(\varphi_{L}\right) E_{0}^{*}-\nu_{n}\left(\mathfrak{m}_{1}\right) E_{p_{1}}^{*}-\sum_{i=2}^{n} \nu_{n}\left(\mathfrak{m}_{i}\right) E_{p_{i}}^{*} \\
& =\nu_{n}\left(\varphi_{L}\right) E_{0}^{*}-\sum_{i=1}^{n} \nu_{n}\left(\mathfrak{m}_{i}\right) E_{p_{i}}^{*},
\end{aligned}
$$

which completes the proof.

Corollary 2.3.4. Let $\nu_{n}$ be a divisorial valuation of $\mathbb{P}^{2}$ and $\mathcal{C}_{\nu_{n}}=\left\{p_{i}\right\}_{i=1}^{n}$ (respectively, $\left.\left\{\nu_{n}\left(\mathfrak{m}_{i}\right)\right\}_{i=0}^{g+1},\left\{\beta_{i}^{\prime}\left(\nu_{n}\right)\right\}_{i=0}^{g+1},\left\{\bar{\beta}_{i}\left(\nu_{n}\right)\right\}_{i=0}^{g+1}\right)$ its configuration of infinitely near points (respectively, its sequence of values, its sequence of Puiseux exponents, its sequence of maximal contact values). Set $\nu$ the special divisorial valuation of $\mathbb{F}_{1}$ defined by $\nu$ as described in Proposition 2.3.3 and denote by $\left\{\beta_{i}^{\prime}(\nu)\right\}_{i=0}^{\hat{g}+1}$ (respectively, $\left\{\bar{\beta}_{i}(\nu)\right\}_{i=0}^{\hat{g}+1}$ ) its sequence of Puiseux exponents (respectively, its sequence of maximal contact values). Then,

(a) If $\beta_{1}^{\prime}\left(\nu_{n}\right) \geq 2$,

- $\beta_{1}^{\prime}(\nu)=\beta_{1}^{\prime}\left(\nu_{n}\right)-1$ and $\beta_{i}^{\prime}(\nu)=\beta_{i}^{\prime}\left(\nu_{n}\right)$, for $2 \leq i<g+2$;

- $\bar{\beta}_{0}(\nu)=\bar{\beta}_{0}\left(\nu_{n}\right)$ and $\bar{\beta}_{i}(\nu)=\bar{\beta}_{i}\left(\nu_{n}\right)-\nu_{n}\left(\mathfrak{m}_{1}\right) \cdot \operatorname{mult}_{p_{1}}\left(\varphi_{l_{i}}\right)$, for $1 \leq i \leq g+1$.

(b) If $1<\beta_{1}^{\prime}\left(\nu_{n}\right)<2$ and $\hat{g}=g$,

- $\beta_{1}^{\prime}(\nu)=\frac{1}{\beta_{1}^{\prime}\left(\nu_{n}\right)-1}$ and $\beta_{i}^{\prime}(\nu)=\beta_{i}^{\prime}\left(\nu_{n}\right)$, for $2 \leq i \leq g+1$;

- $\bar{\beta}_{0}(\nu)=\bar{\beta}_{1}\left(\nu_{n}\right)-\bar{\beta}_{0}\left(\nu_{n}\right), \bar{\beta}_{1}(\nu)=\bar{\beta}_{0}\left(\nu_{n}\right)$ and $\bar{\beta}_{i}(\nu)=\bar{\beta}_{i}\left(\nu_{n}\right)-\nu_{n}\left(\mathfrak{m}_{1}\right) \cdot \operatorname{mult}_{p_{1}}\left(\varphi_{\ell_{i}}\right)$, for $2 \leq i \leq g+1$. 
(c) If $1<\beta_{1}^{\prime}\left(\nu_{n}\right)<2$ and $\hat{g}=g-1$,

- $\beta_{1}^{\prime}(\nu)=\frac{1}{\beta_{1}^{\prime}\left(\nu_{n}\right)-1}+\beta_{2}^{\prime}\left(\nu_{n}\right)-1$ and $\beta_{i}^{\prime}(\nu)=\beta_{i+1}^{\prime}\left(\nu_{n}\right)$, for $2 \leq i<g+1$;

- $\bar{\beta}_{0}(\nu)=\bar{\beta}_{1}\left(\nu_{n}\right)-\bar{\beta}_{0}\left(\nu_{n}\right)$ and $\bar{\beta}_{i}(\nu)=\bar{\beta}_{i+1}\left(\nu_{n}\right)-\nu_{n}\left(\mathfrak{m}_{1}\right) \cdot \operatorname{mult}_{p_{1}}\left(\varphi_{\ell_{i+1}}\right)$, for $1 \leq i \leq g$.

Proof. Following the proof of Proposition 2.3.3, recall that $\mathcal{C}_{\nu}=\left\{q_{i}\right\}_{i=1}^{n-1}$ (respectively, $\left\{\nu\left(\mathfrak{n}_{i}\right)\right\}_{i=1}^{n-1}$ ) denotes the configuration of infinitely near points (respectively, the sequence of values) of $\nu$ which satisfies $q_{i}=p_{i+1}$ (respectively, $\nu\left(\mathfrak{n}_{i}\right)=\nu\left(\mathfrak{m}_{i+1}\right)$ ), for $1 \leq i \leq n-1$. In addition, $E_{p_{i}}$ (respectively, $E_{q_{i}}$ ) is the exceptional divisor created after blowing-up $p_{i}$ (respectively, $q_{i}$ ).

We begin by proving (a). By assumption one has $\nu_{n}\left(\mathfrak{m}_{1}\right)=\nu_{n}\left(\mathfrak{m}_{2}\right)$ and consequently $\hat{g}=g, \bar{\beta}_{0}(\nu)=\bar{\beta}_{0}\left(\nu_{n}\right)$,

$$
\beta_{1}^{\prime}(\nu)=\beta_{1}^{\prime}\left(\nu_{n}\right)-1 \text { and } \beta_{i}^{\prime}(\nu)=\beta_{i}^{\prime}\left(\nu_{n}\right) \text {, for } 2 \leq i<g+2 .
$$

Moreover, by definition $\bar{\beta}_{i}\left(\nu_{n}\right)=\nu_{n}\left(\varphi_{p_{\ell_{i}}}\right)$ (respectively, $\bar{\beta}_{i}(\nu)=\nu_{n}\left(\varphi_{q_{k_{i}}}\right)$ ) where $\varphi_{p_{\ell_{i}}}$ (respectively, $\varphi_{q_{k_{i}}}$ ) is an analytically irreducible germ at $p_{1}$ (respectively, $q_{1}$ ) whose strict transform is transversal to $E_{p_{\ell_{i}}}$ (respectively, $E_{q_{k_{i}}}$ ), being these last two exceptional divisors those corresponding with the dead ends of $\Gamma_{\nu_{n}}^{i}$ and $\Gamma_{\nu}^{i}$, respectively. Moreover, it holds that

$$
q_{k_{i}}=q_{\ell_{i}-1}=p_{\ell_{i}} \text { and } \operatorname{mult}_{q_{j}}\left(\varphi_{q_{k_{i}}}\right)=\operatorname{mult}_{p_{j+1}}\left(\varphi_{p_{\ell_{i}}}\right), \text { for } 1 \leq j \leq n-1,
$$

since $\hat{g}=g$. Therefore,

$$
\begin{aligned}
\bar{\beta}_{i}(\nu)=\nu\left(\varphi_{q_{k_{i}}}\right) & =\sum_{j=1}^{n-1} \nu\left(\mathfrak{n}_{j}\right) \cdot \operatorname{mult}_{q_{j}}\left(\varphi_{q_{k_{i}}}\right) \\
& =\sum_{j=2}^{n} \nu_{n}\left(\mathfrak{m}_{j}\right) \cdot \operatorname{mult}_{p_{j}}\left(\varphi_{p_{\ell_{i}}}\right) \\
& =\nu_{n}\left(\varphi_{p_{\ell_{i}}}\right)-\nu_{n}\left(\mathfrak{m}_{1}\right) \cdot \operatorname{mult}_{p_{1}}\left(\varphi_{p_{\ell_{i}}}\right),
\end{aligned}
$$

which proves (a).

Let us show (b) recall that $\hat{g}=g$. As $1<\beta_{1}^{\prime}\left(\nu_{n}\right)<2, \nu_{n}\left(\mathfrak{m}_{1}\right)>\nu_{n}\left(\mathfrak{m}_{2}\right)$ and then $\bar{\beta}_{0}(\nu)=\nu_{n}\left(\mathfrak{m}_{2}\right)=\bar{\beta}_{1}\left(\nu_{n}\right)-\bar{\beta}_{0}\left(\nu_{n}\right), \bar{\beta}_{1}(\nu)=\bar{\beta}_{0}\left(\nu_{n}\right)$,

$$
\beta_{1}^{\prime}(\nu)=\frac{1}{\beta_{1}^{\prime}\left(\nu_{n}\right)-1} \text { and } \beta_{i}^{\prime}(\nu)=\beta_{i}^{\prime}\left(\nu_{n}\right), \text { for } 2 \leq i \leq g+1,
$$

since $\hat{g}=g$. This proves the statement after noticing that the relation among the maximal contact values of the valuations follows from a similar argument to that developed before.

Finally, we prove (c). In this case, note that $\beta_{1}^{\prime}\left(\nu_{n}\right)=1+1 / a_{2}^{1}$, where $a_{2}^{1} \in \mathbb{Z}_{>0}$. Thus,

$$
\beta_{1}^{\prime}(\nu)=\frac{1}{\beta_{1}^{\prime}\left(\nu_{n}\right)-1}+\beta_{2}^{\prime}\left(\nu_{n}\right)-1 \text { and } \beta_{i}^{\prime}(\nu)=\beta_{i+1}^{\prime}\left(\nu_{n}\right), \text { for } 2 \leq i<g+1 .
$$


In addition, $\bar{\beta}_{0}(\nu)=\nu_{n}\left(\mathfrak{m}_{2}\right)=\bar{\beta}_{1}\left(\nu_{n}\right)-\bar{\beta}_{0}\left(\nu_{n}\right)$ and, taking into account that $q_{k_{i}}=p_{\ell_{i+1}}$, for $1 \leq i \leq g$, and $\operatorname{mult}_{q_{j}}\left(\varphi_{q_{k_{i}}}\right)=\operatorname{mult}_{p_{j+1}}\left(\varphi_{p_{\ell_{i+1}}}\right)$, for $1 \leq j \leq n-1$,

the relation among the maximal contact values of the valuations follows by using an analogous reasoning to that shown previously. This completes the proof.

Remark 2.3.5. Corollary 2.3 .4 provides the following condition

$$
\bar{\beta}_{\hat{g}+1}(\nu)=\bar{\beta}_{g+1}\left(\nu_{n}\right)-\nu_{n}\left(\mathfrak{m}_{1}\right)^{2} .
$$

As a consequence, the following chain of equalities holds:

$$
\begin{aligned}
\Lambda(\nu)^{2} & =2 \nu\left(\varphi_{M_{0}}\right) \nu\left(\varphi_{F_{1}}\right)+\nu\left(\varphi_{F_{1}}\right)^{2}-\bar{\beta}_{\hat{g}+1}(\nu) \\
& =\left(\nu\left(\varphi_{F_{1}}\right)+\nu\left(\varphi_{M_{0}}\right)\right)^{2}-\left(\bar{\beta}_{\hat{g}+1}(\nu)+\nu\left(\varphi_{M_{0}}\right)^{2}\right) \\
& =\left(\nu_{n}\left(\varphi_{L}\right) E_{0}^{*}-\sum_{i=1}^{n} \nu_{n}\left(\mathfrak{m}_{i}\right) E_{i}^{*}\right)^{2},
\end{aligned}
$$

where we have used the fact that $\nu_{n}\left(\varphi_{L}\right)=\nu\left(\varphi_{F_{1}}\right)+\nu\left(\varphi_{M_{0}}\right)$ and $\nu_{n}\left(\mathfrak{m}_{1}\right)=\nu\left(\varphi_{M_{0}}\right)$.

Now we return to our study of surfaces given by special divisorial valuations of Hirzebruch surfaces $\mathbb{F}_{\delta}$ where $\delta$ need not be one. The family of divisors $\left\{\Lambda_{i}\right\}_{i=1}^{n}$ defined in (2.3) is important in this section because it has interesting properties which are suitable to achieve our propose, as we will see in the following result.

Lemma 2.3.6. Let $\nu_{n}$ be a special divisorial valuation of $\mathbb{F}_{\delta}$. Then, under the above notation, it holds that $\Lambda_{1}^{2} \geq 0$, and the inequality $\Lambda_{i}^{2} \geq 0$ for some index $i \in\{2,3, \ldots, n\}$ implies:

(a) $\Lambda_{i}^{2}>0$, if $p_{i}$ is a satellite point of the configuration $\mathcal{C}_{\nu_{n}}$.

(b) $\Lambda_{i-1}^{2} \geq 0$ and, when $\Lambda_{i-1}^{2}=0$, the point $p_{i}$ is satellite and the point $p_{i-1}$ is free.

Proof. The self-intersection of the divisor $\Lambda_{1}$ satisfies $\Lambda_{1}^{2}=1+\delta$ when $p_{1}$ is a special point and also when $\delta=0$. Otherwise, $\Lambda_{1}^{2}=\delta-1$.

For proving the remaining statements, we can assume, without loss of generality, that $i=n \geq 2$.

We are going to prove the result when $p_{1}$ is a special point. Otherwise, the proof is the same after setting $\delta=0$ or $a_{n}=0$.

We start with the proof of $(a)$ for which we will use some properties of the set of maximal contact values of $\nu_{n},\left\{\bar{\beta}_{j}\left(\nu_{n}\right)\right\}_{j=0}^{g+1}$. We divide this proof in two cases.

Case 1(a): $g>1$. Reasoning by contradiction and taking into account that the point $p_{n}$ is satellite, we get that

$$
0=\Lambda_{n}^{2}=2 a_{n} b_{n}+\delta b_{n}^{2}-e_{g-1}\left(\nu_{n}\right) \bar{\beta}_{g}\left(\nu_{n}\right)=e_{g-1}\left(\nu_{n}\right)\left[\frac{2 a_{n} b_{n}+\delta b_{n}^{2}}{e_{g-1}\left(\nu_{n}\right)}-\bar{\beta}_{g}\left(\nu_{n}\right)\right]
$$


where $e_{g-1}\left(\nu_{n}\right)=\operatorname{gcd}\left(\bar{\beta}_{0}\left(\nu_{n}\right), \bar{\beta}_{1}\left(\nu_{n}\right), \ldots, \bar{\beta}_{g-1}\left(\nu_{n}\right)\right)$ (see Proposition 1.3.5). Since both $a_{n}$ and $b_{n}$ are either a multiple of $\bar{\beta}_{0}\left(\nu_{n}\right)$ or $\bar{\beta}_{1}\left(\nu_{n}\right)$, the first addend in the brackets is a multiple of $e_{g-1}\left(\nu_{n}\right)$, which gives a contradiction because $\operatorname{gcd}\left(e_{g-1}\left(\nu_{n}\right), \bar{\beta}_{g}\left(\nu_{n}\right)\right)$ equals 1 .

Case 2(a): $g=1$. We distinguish three sub-cases: The first one when the values $a_{n}$ and $b_{n}$ are divisible by $\bar{\beta}_{0}\left(\nu_{n}\right)$. Then $e_{g-1}\left(\nu_{n}\right)=e_{0}\left(\nu_{n}\right)=\bar{\beta}_{0}\left(\nu_{n}\right)$ and the proof follows as above. The second one when the value $a_{n}$ satisfies $a_{n}=\bar{\beta}_{1}\left(\nu_{n}\right)$; then $\Lambda_{n}^{2}=\bar{\beta}_{0}\left(\nu_{n}\right)\left(2 \bar{\beta}_{1}\left(\nu_{n}\right)+\bar{\beta}_{0}\left(\nu_{n}\right) \delta-\bar{\beta}_{1}\left(\nu_{n}\right)\right)>0$. Otherwise, it holds that

$$
\Lambda_{n}^{2}=\bar{\beta}_{1}\left(\nu_{n}\right)\left(2 \bar{\beta}_{0}\left(\nu_{n}\right)+\bar{\beta}_{1}\left(\nu_{n}\right) \delta-\bar{\beta}_{0}\left(\nu_{n}\right)\right)>0,
$$

which concludes the proof of $(a)$.

Now we prove $(b)$. Again we can suppose that $i=n$. We also assume that the point $p_{n}$ is satellite (otherwise $\Lambda_{n-1}^{2}>0$ by the Noether formula). Denote by $\nu_{n-1}$ the divisorial valuation defined by the divisor $E_{n-1}$. Let $\left\{\bar{\beta}_{j}\left(\nu_{n-1}\right)\right\}_{j=0}^{\widehat{g}+1}$ be the sequence of maximal contact values of $\nu_{n-1}$,

$$
e_{g-1}\left(\nu_{n-1}\right)=\operatorname{gcd}\left(\bar{\beta}_{0}\left(\nu_{n-1}\right), \bar{\beta}_{1}\left(\nu_{n-1}\right), \ldots, \bar{\beta}_{g-1}\left(\nu_{n-1}\right)\right)
$$

and $e:=e_{\widehat{g}-1}\left(\nu_{n-1}\right) / e_{\widehat{g}-1}\left(\nu_{n}\right)$. Consider two cases with two sub-cases.

Case 1(b): $g=\hat{g}$. Assume first that $g>1$. From the following equality, which is proved in [60, Lemma 2],

$$
\left|\bar{\beta}_{g}\left(\nu_{n-1}\right)-e \bar{\beta}_{g}\left(\nu_{n}\right)\right|=\frac{1}{e_{g-1}\left(\nu_{n}\right)},
$$

one can deduce that

$$
-\frac{e_{g-1}\left(\nu_{n}\right) \bar{\beta}_{g}\left(\nu_{n-1}\right)}{e} \geq-\frac{1}{e}-e_{g-1}\left(\nu_{n}\right) \bar{\beta}_{g}\left(\nu_{n}\right) .
$$

In this case both valuations $\nu_{n}$ and $\nu_{n-1}$ are defined by satellite points, therefore $a_{n-1}=e a_{n}, b_{n-1}=e b_{n}, \bar{\beta}_{g+1}\left(\nu_{n}\right)=e_{g-1}\left(\nu_{n}\right) \bar{\beta}_{g}\left(\nu_{n}\right)$ and

$$
\bar{\beta}_{g+1}\left(\nu_{n-1}\right)=e_{g-1}\left(\nu_{n-1}\right) \bar{\beta}_{g}\left(\nu_{n-1}\right)
$$

by Equality (1.5) and Corollary 1.3.7. As a consequence

$$
\begin{aligned}
\Lambda_{n-1}^{2} & =e^{2}\left[2 a_{n} b_{n}+\delta b_{n}^{2}-\frac{e_{g-1}\left(\nu_{n}\right) \bar{\beta}_{g}\left(\nu_{n-1}\right)}{e}\right] \\
& \geq e^{2}\left[2 a_{n} b_{n}+\delta b_{n}^{2}-\frac{1}{e}-e_{g-1}\left(\nu_{n}\right) \bar{\beta}_{g}\left(\nu_{n}\right)\right] \\
& =e^{2}\left[\Lambda_{n}^{2}-\frac{1}{e}\right]>0,
\end{aligned}
$$

where the first inequality is deduced from (2.6) and the last one holds since $\Lambda_{n}^{2}>$ $e_{g-1}\left(\nu_{n}\right)>1 / e$. 
To conclude the proof in this case, it remains to study what happens when $g=1$. We consider the same subcases as above. The first one where the values $a_{n}$ and $b_{n}$ are both divisible by $\bar{\beta}_{0}\left(\nu_{n}\right)$ and the fact $\Lambda_{n-1}^{2}>0$ can be proved as before. The second one where the value $a_{n}$ equals $\bar{\beta}_{1}\left(\nu_{n}\right)$, then one has that

$$
\begin{aligned}
\Lambda_{n-1}^{2} & =2 \bar{\beta}_{0}\left(\nu_{n-1}\right) \bar{\beta}_{1}\left(\nu_{n-1}\right)+\delta \bar{\beta}_{0}\left(\nu_{n-1}\right)^{2}-\bar{\beta}_{2}\left(\nu_{n-1}\right) \\
& =\bar{\beta}_{0}\left(\nu_{n-1}\right)\left(2 \bar{\beta}_{1}\left(\nu_{n-1}\right)+\delta \bar{\beta}_{0}\left(\nu_{n-1}\right)-\bar{\beta}_{1}\left(\nu_{n-1}\right)\right) \\
& =\bar{\beta}_{0}\left(\nu_{n-1}\right)\left(\bar{\beta}_{1}\left(\nu_{n-1}\right)+\delta \bar{\beta}_{0}\left(\nu_{n-1}\right)\right)>0 .
\end{aligned}
$$

Otherwise, one has $\Lambda_{n-1}^{2}=\bar{\beta}_{1}\left(\nu_{n-1}\right)\left(\bar{\beta}_{0}\left(\nu_{n-1}\right)+\delta \bar{\beta}_{1}\left(\nu_{n-1}\right)\right)>0$ and the proof ends.

Case 2(b): $\widehat{g}=g-1$. When $g>1$, it holds

$$
\bar{\beta}_{\hat{g}+1}\left(\nu_{n-1}\right)=\frac{\bar{\beta}_{g+1}\left(\nu_{n}\right)+2}{4}
$$

and thus

$$
\Lambda_{n-1}^{2}=\frac{1}{4}\left(2 a_{n} b_{n}+\delta b_{n}^{2}-\bar{\beta}_{g+1}\left(\nu_{n}\right)-2\right)=\frac{1}{4} \Lambda_{n}^{2}-\frac{1}{2} \geq 0,
$$

because $\Lambda_{n}^{2} \geq 2$.

Finally we must assume that $g=1$ and, as above, when the values $a_{n}$ and $b_{n}$ are divisible by $\bar{\beta}_{0}\left(\nu_{n}\right)=2, \Lambda_{n-1}^{2} \geq 0$. When $a_{n}=\bar{\beta}_{1}\left(\nu_{n}\right), \Lambda_{n-1}^{2}=\bar{\beta}_{1}\left(\nu_{n-1}\right)+\delta \geq 0$, and otherwise,

$$
\Lambda_{n-1}^{2}=2 \bar{\beta}_{1}\left(\nu_{n-1}\right)+\delta \bar{\beta}_{1}\left(\nu_{n-1}\right)^{2}-\bar{\beta}_{1}\left(\nu_{n-1}\right) \geq 0,
$$

which concludes the proof.

Recall that $\nu_{n}$ is a special divisorial valuation of $\mathbb{F}_{\delta}$ and $Z$ is the surface defined by $\nu_{n}$. The next theorem provides equivalent conditions to the fact that $\nu_{n}$ is non-positive at infinity. This result uses the values $a_{n}$ and $b_{n}$ and the divisor $\Lambda_{n}$ introduced in Proposition 2.3.1.

Theorem 2.3.7. Let $\nu_{n}$ be a special divisorial valuation of the function field of $\mathbb{F}_{\delta}$ centered at $\mathcal{O}_{\mathbb{F}_{\delta}, p}$. Set $Z$ the surface that $\nu_{n}$ defines. Consider the divisor $\Lambda_{n}$ given in (2.3) and the last maximal contact value of $\nu_{n}, \bar{\beta}_{g+1}\left(\nu_{n}\right)$. Then the following conditions are equivalent:

(a) The valuation $\nu_{n}$ is non-positive at infinity.

(b) The divisor $\Lambda_{n}$ is nef.

(c) The inequality $2 a_{n} b_{n}+\delta b_{n}^{2} \geq \bar{\beta}_{g+1}\left(\nu_{n}\right)$ holds.

(d) The cone of curves $\mathrm{NE}(Z)$ is generated by the classes of the strict transforms on $Z$ of the fiber passing through $p$, the section $M_{0}$ and the irreducible exceptional divisors associated with the map $\pi$ given by $\nu_{n}$. 
Proof. Our first step is to prove the equivalence between (a) and (b), and we start by proving that (b) implies (a). We assume firstly that $\delta>0$ and $p=p_{1}$ is a special point. We are going to use the notation introduced in Subsection 1.6.1. Without loss of generality, suppose that the special point $p$ has coordinates $(1: 0 ; 1,0)$. The point $p$ belongs to the fiber $F_{1}$ whose equation is $X_{1}=0$, and the special section $M_{0}$ is defined by the equation $Y_{1}=0$. Set $U_{00}$ the affine open set of $\mathbb{F}_{\delta}$ given by $X_{0} \neq 0$ and $Y_{0} \neq 0$, whose associated affine coordinates are $\{u, v\}=\left\{\frac{X_{1}}{X_{0}}, \frac{X_{0}^{\delta} Y_{1}}{Y_{0}}\right\}$. Consider also the affine open set of $\mathbb{F}_{\delta}, U_{11}$, defined by $X_{1} \neq 0$ and $Y_{1} \neq 0$, with coordinates $\{x, y\}=\left\{\frac{X_{0}}{X_{1}}, \frac{Y_{0}}{X_{1}^{\delta} Y_{1}}\right\}$. It holds that $p \in U_{00}$ and $F_{1}$ and $M_{0}$ have local equations $u=0$ and $v=0$, respectively. Denote by $\mathcal{P}$ the set of non-constant functions in $\mathcal{O}_{\mathbb{F}_{\delta}}\left(U_{11}\right)$ (up to multiplication by a nonzero element of $k$ ) such that neither $x$ nor $y$ divide them. In terms of the coordinates $\{u, v\}, f \in \mathcal{P}$ can be expressed as

$$
f(x, y)=f\left(1 / u, 1 / u^{\delta} v\right)=\frac{h_{f}(u, v)}{u^{\operatorname{deg}_{1}\left(h_{f}\right)+\delta \operatorname{deg}_{2}\left(h_{f}\right)} v^{\operatorname{deg}_{2}\left(h_{f}\right)}},
$$

where $h_{f}(u, v) \in \mathcal{O}_{\mathbb{F}_{\delta}}\left(U_{00}\right)$. The bi-homogeneous polynomial

$$
X_{0}^{\operatorname{deg}_{1}\left(h_{f}\right)} Y_{0}^{\operatorname{deg}_{2}\left(h_{f}\right)} \cdot h_{f}\left(\frac{X_{1}}{X_{0}}, \frac{X_{0}^{\delta} Y_{1}}{Y_{0}}\right)
$$

defines a curve $C_{f}$ on the surface $\mathbb{F}_{\delta}$ of degree $\left(\operatorname{deg}_{1}\left(h_{f}\right), \operatorname{deg}_{2}\left(h_{f}\right)\right)$ and, if $F^{\prime}$ and $M^{\prime}$ are the fiber and the section on $\mathbb{F}_{\delta}$ with equations $X_{0}=0$ and $Y_{0}=0$, it holds that the map $f \rightarrow C_{f}$ defines a one-to-one correspondence between $\mathcal{P}$ and the set of the curves on $\mathbb{F}_{\delta}$ containing no curve in $\left\{F_{1}, F^{\prime}, M_{0}, M^{\prime}\right\}$ as a component. Now, the conditions $\Lambda_{n}$ nef and (2.7) show that

$$
\begin{aligned}
0 & \leq \Lambda_{n} \cdot C_{f}=\Lambda_{n} \cdot\left[\operatorname{deg}_{1}\left(h_{f}\right) F^{*}+\operatorname{deg}_{2}\left(h_{f}\right) M^{*}-\sum_{i=1}^{n} \operatorname{mult}_{p_{i}}\left(h_{f}\right) E_{i}^{*}\right] \\
& =-\left[-\left(\operatorname{deg}_{1}\left(h_{f}\right)+\operatorname{deg}_{2}\left(h_{f}\right) \delta\right) \nu_{n}(u)-\operatorname{deg}_{2}\left(h_{f}\right) \nu_{n}(v)+\nu_{n}\left(h_{f}\right)\right]=-\nu_{n}(f) .
\end{aligned}
$$

Thus, to finish the proof of (a) in this case ( $p$ is a special point), it only remains to assume that either $x$ or $y$ or both are factors of $f$. Then the proof follows from the existence of non-negative integers $\alpha, \beta$ with $\alpha+\beta \neq 0$ and $f_{1} \in \mathcal{P}$ such that

$$
\nu_{n}(f)=\nu_{n}\left(x^{\alpha} y^{\beta} f_{1}\right)=-(\alpha+\beta \delta) \nu_{n}(u)-\beta \nu_{n}(v)+\nu_{n}\left(f_{1}\right) \leq 0 .
$$

If $\delta=0$ the proof is analogous, and the non-positivity of $\nu_{n}$ for the case when $p$ is a general point can be proved in a similar way after assuming that $p$ has coordinates $(0: 1 ; 0,1)$ and considering local coordinates $\{u, v\}=\left\{\frac{X_{0}}{X_{1}}, \frac{Y_{0}}{X_{1}^{\delta} Y_{1}}\right\}$ in the affine open set $U_{11}$ and $\{x, y\}=\left\{\frac{X_{1}}{X_{0}}, \frac{Y_{0}}{X_{0}^{\delta} Y_{1}}\right\}$ in $U_{01}$.

Now we are going to prove that (a) implies (b). Assume by contradiction that the divisor $\Lambda_{n}$ is not nef and, therefore, that there exists an effective divisor $C$ such that $\Lambda_{n} \cdot C<0$. This implies that, with the above notation, if $p$ is a special point 
-or $p \in \mathbb{F}_{0^{-}}$, (respectively, $p$ is a general point), then there exists $f \in \mathcal{O}_{\mathbb{F}_{\delta}}\left(U_{11}\right)$ (respectively, $\left.f \in \mathcal{O}_{\mathbb{F}_{\delta}}\left(U_{01}\right)\right)$ such that $-\nu_{n}(f)=\Lambda_{n} \cdot C<0$, a contradiction.

The fact that (b) implies (c) follows easily from previous computations given in Remark 2.3.2.

Let us prove that (d) can be deduced from (c). Fix any ample divisor $H$ on the surface $Z$ and consider the set

$$
A(Z):=\left\{[D] \in \operatorname{Pic}_{\mathbb{R}}(Z) \mid[D]^{2} \geq 0 \text { and }[H] \cdot[D] \geq 0\right\} .
$$

Recall that the above defined cone $S_{1}(Z)$ is generated by the classes $\left[\tilde{F}_{1}\right],\left[\tilde{M}_{0}\right]$ and $\left[E_{i}\right], 1 \leq i \leq n$, and we are going to prove that

$$
\overline{\mathrm{NE}}(Z)=S_{1}(Z)+S_{1}^{\vee}(Z)=\mathrm{NE}(Z)
$$

and

$$
S_{1}^{\vee}(Z) \subseteq A(Z) \subseteq S_{1}(Z)
$$

hold, which shows (d). Our Condition (c) means that $\Lambda_{n}^{2} \geq 0$ and by Lemma 2.3.6, one has that $\Lambda_{i}^{2} \geq 0,1 \leq i \leq n-1$. Proposition 2.3.1 proves the first inclusion in (2.9) and the last one follows from the first one and the equality $A(Z)^{\vee}=A(Z)$ given in Proposition 1.4.14.

It remains to prove the chain of equalities (2.8). First, notice that $A(Z) \subseteq \overline{\mathrm{NE}}(Z)$ by Proposition 1.4.13. Thus $S_{1}^{\vee}(Z) \subseteq \overline{\mathrm{NE}}(Z)$. Now, if $[C]$ is the class of an irreducible curve on $Z$ and it is not one of the given generators of $S_{1}(Z)$, then $[C] \in S_{1}^{\vee}(Z)$ because otherwise $[C] \cdot[D]<0$ for $[D] \in S_{1}(Z)$ and $C$ and $D$ would have a common component. Therefore we have proved (2.8) with inclusions $\supseteq$ instead of equalities. Taking topological closures we deduce that (2.8) holds.

Finally (d) implies (b) by Proposition 2.3.1, which concludes the proof.

Remark 2.3.8. Taking into account the proof of Proposition 2.3.3 and Remark 2.3.5, one can state Theorem 2.1.2 as a particular case of Theorem 2.3.7. Moreover, a divisorial valuation of $\mathbb{P}^{2}$ is NPI if and only if the corresponding special divisorial valuation of $\mathbb{F}_{1}$ is NPI.

Remark 2.3.9. By Proposition 2.3.1, Proposition 2.3.3, Theorem 2.3.7 and Remark 2.3.8, it holds that the nef cone $\operatorname{Nef}(Z)$ of $Z$ is generated by $\left[F^{*}\right],\left[M^{*}\right]$ and $\left[\Lambda_{i}\right]$, $1 \leq i \leq n$, when $\nu_{n}$ is a NPI special divisorial valuation of $\mathbb{F}_{\delta}$, and by $\left[E_{0}^{*}\right]$ and $\left[D_{i}\right]$, where $D_{i}=\nu_{n}\left(\varphi_{L}\right) E_{0}^{*}-\sum_{i=1}^{n} \nu_{n}\left(\mathfrak{m}_{i}\right) E_{i}^{*}$ and $1 \leq i \leq n$, if $\nu_{n}$ is a NPI divisorial valuation of $\mathbb{P}^{2}$.

Corollary 2.3.10. Under the notation in Theorem 2.3.7, assume that $\nu_{n}$ is NPI and, for $1 \leq i \leq n-1$, denote by $\nu_{i}$ the special divisorial valuation of $\mathbb{F}_{\delta}$ defined by the divisor $E_{i}$ created by each map $\pi_{i}$ appearing in the sequence (2.2) defined by $\nu_{n}$. Then the valuation $\nu_{i}$ is also non-positive at infinity. 
Proof. By hypothesis and Lemma 2.3.6, one has that $\Lambda_{i}^{2} \geq 0$, for $i \in\{1,2 \ldots, n-1\}$. As a result, following Remark 2.3.2 and Theorem 2.3.7, $\nu_{i}$ is NPI for $1 \leq i \leq n-1$.

Corollary 2.3.11. Keeping the notation as in Theorem 2.3.7, if $\Lambda_{n}$ is a nef divisor, then every $\Lambda_{i}$ is effective, for $1 \leq i \leq n$. In particular,

$$
\Lambda_{i}=\left(\Lambda_{i} \cdot M^{*}\right) \tilde{F}_{1}+\left(\Lambda_{i} \cdot F^{*}\right) \tilde{M}_{0}+\sum_{j=1}^{n}\left(\Lambda_{i} \cdot \Lambda_{j}\right) E_{j} .
$$

Proof. It is clear that $\Lambda_{i}$ is nef and its self-intersection is non-negative by Corollary 2.3.10. Moreover, the set $\left\{\left[F^{*}\right],\left[M^{*}\right]\right\} \cup\left\{\left[\Lambda_{i}\right]\right\}_{i=1}^{n}$ is the dual basis of the basis $\left\{\left[\tilde{F}_{1}\right],\left[\tilde{M}_{0}\right]\right\} \cup\left\{\left[E_{i}\right]\right\}_{i=1}^{n}$ of $\operatorname{Pic}_{\mathbb{R}}(Z)$ by Proposition 2.3.1 and the classes of the divisors in the set $\left\{F^{*}, M^{*}\right\} \cup\left\{\Lambda_{i}\right\}_{i=1}^{n}$ belong to $A(Z) \subset \overline{\mathrm{NE}}(Z)$. As a consequence, the divisor $\Lambda_{i}$ can be written as

$$
\Lambda_{i}=\alpha_{i 1} \tilde{F}+\alpha_{i 2} \tilde{M}_{0}+\sum_{j=1}^{n} \beta_{i j} E_{j}
$$

where $\alpha_{i 1}=\Lambda_{i} \cdot M^{*} \geq 0, \alpha_{i 2}=\Lambda_{i} \cdot F^{*} \geq 0$ and $\beta_{i j}=\Lambda_{i} \cdot \Lambda_{j} \geq 0$, for $1 \leq j \leq n$, which concludes the proof.

Now we state a result which allows us to define a sequence of non-positive at infinity special valuations of $\mathbb{F}_{\delta}, \delta \geq 0$, which approaches a non-positive at infinity special irrational valuation of $\mathbb{F}_{\delta}$.

Corollary 2.3.12. Let $\nu_{n}$ be a non-positive at infinity special divisorial valuation of $\mathbb{F}_{\delta}$ and $\mathcal{C}_{\nu_{n}}=\left\{p_{i}\right\}_{i=1}^{n}$ its configuration of infinitely near points. Set a special divisorial valuation $\nu_{m}$ such that its configuration $\mathcal{C}_{\nu_{m}}=\left\{q_{i}\right\}_{i=1}^{m}, n<m$, satisfies that $\mathcal{C}_{\nu_{n}} \subset \mathcal{C}_{\nu_{m}}$ and the points $q_{i}, n+1 \leq i \leq m$, are satellite. Then, the valuation $\nu_{m}$ is non-positive at infinity.

Proof. Denote by $\left\{\bar{\beta}_{i}\left(\nu_{n}\right)\right\}_{i=1}^{g+1}$ (respectively, $\left\{\bar{\beta}_{i}\left(\nu_{m}\right)\right\}_{i=1}^{\hat{g}+1}$ ) the sequence of maximal contact values of $\nu_{n}$ (respectively, $\left.\nu_{m}\right)$. Define $e_{g-1}\left(\nu_{n}\right):=\operatorname{gcd}\left(\bar{\beta}_{1}\left(\nu_{n}\right), \bar{\beta}_{2}\left(\nu_{n}\right), \ldots\right.$, $\left.\bar{\beta}_{g-1}\left(\nu_{n}\right)\right)$ and $e_{\hat{g}-1}\left(\nu_{m}\right)=\operatorname{gcd}\left(\bar{\beta}_{1}\left(\nu_{m}\right), \bar{\beta}_{2}\left(\nu_{m}\right), \ldots, \bar{\beta}_{\hat{g}-1}\left(\nu_{m}\right)\right)$.

Notice that it holds that

$$
e_{\hat{g}-1}\left(\nu_{m}\right) \bar{\beta}_{g+1}\left(\nu_{n}\right) \geq \bar{\beta}_{\hat{g}}\left(\nu_{m}\right) .
$$

In fact, consider that $p_{n}$ is a free point and then the inequality holds by the Noether formula and the inequality $e_{\hat{g}-1}\left(\nu_{m}\right) \operatorname{mult}_{p_{n}}\left(\varphi_{n}\right) \geq \operatorname{mult}_{p_{n}}\left(\varphi_{m}\right)$. The satellite case follows from the previous one.

By Theorem 2.3.7 and Remark 2.3.2, the valuation $\nu_{m}$ is non-positive at infinity if and only if $2 \nu_{m}\left(\varphi_{M_{0}}\right) \nu_{m}\left(\varphi_{F_{1}}\right)+\delta \nu_{m}\left(\varphi_{F_{1}}\right)^{2}-\bar{\beta}_{\hat{g}+1}\left(\nu_{m}\right) \geq 0$. It is easy to check that the last condition holds since

$$
\begin{aligned}
2 \nu_{m}\left(\varphi_{M_{0}}\right) \nu_{m}\left(\varphi_{F_{1}}\right)+\delta \nu_{m}\left(\varphi_{F_{1}}\right)^{2} & =e_{\hat{g}-1}^{2}\left(\nu_{m}\right)\left(2 \nu_{n}\left(\varphi_{M_{0}}\right) \nu_{n}\left(\varphi_{F_{1}}\right)+\delta \nu_{n}\left(\varphi_{F_{1}}\right)^{2}\right) \\
& \geq e_{\hat{g}-1}^{2}\left(\nu_{m}\right) \bar{\beta}_{g+1}\left(\nu_{n}\right) \geq \bar{\beta}_{\hat{g}+1}\left(\nu_{m}\right),
\end{aligned}
$$


by Corollary 1.3.7, Equation (2.10) and the fact that $\nu_{n}$ is non-positive at infinity, which completes the proof.

Notice that one gets a similar property for non-positive at infinity divisorial valuation of $\mathbb{P}^{2}$ by Remark 2.3.8.

Next we show an example of the previously stated results.

Example 2.3.13. Let $\nu$ be a special divisorial valuation of $\mathbb{F}_{2}$ whose sequence of maximal contact values is $\{16,24,60,131,524\} . \Gamma_{\nu}$ (Figure 2.1) is its dual graph. Set $\mathcal{C}_{\nu}=\left\{p_{i}\right\}_{i=1}^{12}$ (where $p=p_{1}$ ) its configuration of infinitely near points and assume that $p$ is a special point, $F_{1}$ is the fiber going through $p$ and the strict

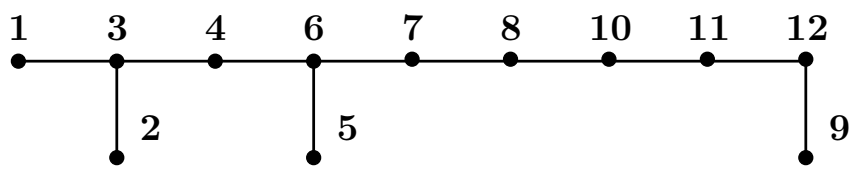

Figure 2.1: Dual graph of $\nu$ in Example 2.3.13

transform of $M_{0}$ passes through $p_{2}$. Therefore, it holds that $a_{12}=\left(\varphi_{M_{0}}, \varphi_{12}\right)_{p}=24$, $b_{12}=\left(\varphi_{F_{1}}, \varphi_{12}\right)_{p}=16$ and $2 a_{12} b_{12}+\delta b_{12}^{2}=1280>524=\bar{\beta}_{g+1}(\nu)$. Consequently, by Theorem 2.3.7, the cone of curves $\mathrm{NE}(Z)$ of $Z$ defined by $\nu$ is generated by $\left[\tilde{F}_{1}\right],\left[\tilde{M}_{0}\right]$ and $\left\{\left[E_{i}\right]\right\}_{i=1}^{12}$ and the divisors $\Lambda_{i}, 1 \leq i \leq 12$, defined in Proposition 2.3.1 are nef.

To finish this section we provide a result that characterizes the fact that a special divisorial valuation of $\mathbb{F}_{\delta}$ is negative at infinity. Also, by Proposition 2.3.3, Theorem 2.1.3 is a particular case of that result.

Theorem 2.3.14. Under the notation in Theorem 2.3.7, the following conditions are equivalent:

(a) The valuation $\nu_{n}$ is negative at infinity.

(b) It holds that either $2 a_{n} b_{n}+b_{n}^{2} \delta>\bar{\beta}_{g+1}\left(\nu_{n}\right)$, or $2 a_{n} b_{n}+b_{n}^{2} \delta=\bar{\beta}_{g+1}\left(\nu_{n}\right)$ and the Iitaka dimension of the divisor $\Lambda_{n}$ vanishes.

(c) The inequality $\Lambda_{n} \cdot \tilde{C}>0$ holds for the strict transform on $Z, \tilde{C}$, of any curve $C$ on $\mathbb{F}_{\delta}, C \neq F_{1}, M_{0}$.

Proof. For a start, we recall that the Iitaka dimension [72] of a divisor $D$ on $Z$ is the maximum of the projective dimensions of the closures of the images of the rational maps defined by the complete linear systems $|n D|$, when $n$ runs over those positive integers $m$ such that $H^{0}\left(Z, \mathcal{O}_{Z}(m D)\right) \neq 0$.

We assume that $p_{1}$ is a special point. The other cases can be proved similarly. Consider the same notations as in the proof of Theorem 2.3.7. Assume also, without loss of generality, that $p_{1}$ has coordinates $(1: 0 ; 1,0)$. 
We start by proving by contradiction that (b) can be deduced from (a). Hence, assume that (a) holds but (b) is false (what means, taking into account Theorem 2.3.7, that $\Lambda_{n}^{2}=0$ and $\operatorname{dim}\left|m \Lambda_{n}\right|>0$ for $m$ large enough). Therefore, there exists $f \in \mathcal{P}$ such that the class of $m \Lambda_{n}-\tilde{C}_{f}$ is effective for $m$ large enough. This implies that

$$
0 \leq \Lambda_{n} \cdot\left(m \Lambda_{n}-\tilde{C}_{f}\right)=m \Lambda_{n}^{2}-\Lambda_{n} \cdot \tilde{C}_{f}=-\Lambda_{n} \cdot \tilde{C}_{f} .
$$

Hence $0=\Lambda_{n} \cdot \tilde{C}_{f}=-\nu_{n}(f)$ because $\Lambda_{n}$ is nef (by Theorem 2.3.7), and this fact contradicts (a).

To prove that (b) implies (c), we reason again by contradiction and consider $C$ an integral curve on $\mathbb{F}_{\delta}$ different from $F_{1}$ and $M_{0}$, and such that $\Lambda_{n} \cdot \tilde{C} \leq 0$. In fact $\Lambda_{n} \cdot \tilde{C}=0$ because, by Theorem 2.3.7, $\Lambda_{n}$ is nef. Let $\mathcal{F}$ be the face of the cone of curves of $Z$ spanned by the classes $\left[\tilde{F}_{1}\right],\left[\tilde{M}_{0}\right],\left[E_{1}\right], \ldots,\left[E_{n-1}\right]$, that is, $\mathcal{F}=\left[\Lambda_{n}\right]^{\perp} \cap \mathrm{NE}(Z)$. It is clear that $[\tilde{C}] \in \mathcal{F}$ and, since the extremal rays of $\mathrm{NE}(Z)$ are generated by classes of irreducible curves with negative self-intersection, $\tilde{C}^{2}=0$. $\tilde{C}$ is nef, so $[\tilde{C}]^{\perp} \cap \mathrm{NE}(Z)$ is a face of $\mathrm{NE}(Z)$ which contains $[\tilde{C}]$ and, then, it must coincide with $\left[\Lambda_{n}\right]^{\perp} \cap \mathrm{NE}(Z)$. Indeed, this is a consequence of the fact that, in suitable coordinates, $A(Z)$ is the projective cone over an Euclidean ball $B$ (by the Hodge index theorem (Theorem 1.4.11)) and $B$ is strictly convex. Then, $\tilde{C}$ is linearly equivalent to a multiple of $\Lambda_{n}$ and, by Corollary 2.3.11, we get a contradiction.

To finish, the fact that (c) implies (a) can be proved as in Theorem 2.3.7 when proving that (b) implies (a).

\subsection{The cone of curves of a surface defined by an NPI non-special divisorial valuation of a Hirzebruch sur- face}

In this section we only consider non-special divisorial valuations $\nu_{n}$ of $\mathbb{F}_{\delta}, \delta>0$. As in Section 2.3, we are going to provide several equivalent conditions to the fact that $\nu_{n}$ is non-positive or negative at infinity.

Notice that, since our divisorial valuations are non-special, by Proposition 2.2.2, one has that two important properties hold: On the one hand, at least the points $p=p_{1}, p_{2}, \ldots, p_{\delta+1}$ of the configuration of infinitely near points are free. On the other hand, there exists a unique integral curve of degree $(0,1)$ which is linearly equivalent to $M$ whose strict transform on $Z$ has negative self-intersection (see Proposition 2.2.2). This curve will be denoted by $M_{1}$. Consequently, $\left[\tilde{M}_{1}\right]$ generates an extremal ray of $\operatorname{NE}(Z)$ and $\overline{\mathrm{NE}}(Z)$ as well as $\left[\tilde{F}_{1}\right]$ and $\left[\tilde{M}_{0}\right]$, where $F_{1}$ is the fiber passing through the point $p$ and $M_{0}$ the special section.

Following Section 2.3, denote by $S_{2}(Z)$ the strongly convex cone of $\operatorname{Pic}_{\mathbb{R}}(Z)$ generated by the classes of the divisors of the set $\left\{\tilde{F}_{1}, \tilde{M}_{0}, \tilde{M}_{1}\right\} \cup\left\{E_{1}\right\}_{i=1}^{n}$. We will 
describe its dual cone $S_{2}^{\vee}(Z)$ in Proposition 2.4.2. Before stating this result, we prove a useful lemma.

Lemma 2.4.1. The class of the strict transform of $M_{1}$ on $Z,\left[\tilde{M}_{1}\right]$, can be written as

$$
\begin{aligned}
{\left[\tilde{M}_{1}\right]=\delta\left[\tilde{F}_{1}\right]+} & {\left[\tilde{M}_{0}\right]+(\delta-1)\left[E_{1}\right]+(\delta-2)\left[E_{2}\right]+\cdots } \\
& +\left[E_{\delta-1}\right]+d_{\delta+1}\left[E_{\delta+1}\right]+\cdots+d_{n}\left[E_{n}\right]
\end{aligned}
$$

where $d_{i} \in \mathbb{Z}$ and $d_{i} \leq-1$ for all $i=\delta+1, \delta+2, \ldots, n$.

Proof. It is clear that we can write $\left[\tilde{M}_{1}\right]$ as

$$
\left[\tilde{M}_{1}\right]=d_{01}\left[\tilde{F}_{1}\right]+d_{02}\left[\tilde{M}_{0}\right]+d_{1}\left[E_{1}\right]+\cdots+d_{n}\left[E_{n}\right]
$$

for some values $d_{01}, d_{02}, d_{i} \in \mathbb{Z}, 1 \leq i \leq n$. Now, using the equalities

$$
\left[\tilde{F}_{1}\right]=\left[F^{*}\right]-\left[E_{1}^{*}\right],\left[\tilde{M}_{0}\right]=\delta\left[F^{*}\right]+\left[M^{*}\right] \text { and }\left[E_{i}\right]=\left[E_{i}^{*}\right]-\sum_{p_{j} \rightarrow p_{i}}\left[E_{j}^{*}\right]
$$

we can compare the above expression with the equality $\left[\tilde{M}_{1}\right]=\left[M^{*}\right]-\sum_{j=1}^{i_{M_{1}}}\left[E_{j}^{*}\right]$, where $i_{M_{1}}$ is the index of the last point of the configuration of infinitely near points given by $\nu, \mathcal{C}_{\nu}$, through which $\tilde{M}_{1}$ goes. This gives rise to a system of linear equations in the variables $d_{01}, d_{02},\left\{d_{i}\right\}_{i=1}^{n}$, whose first equations are

$$
\begin{array}{r}
d_{01}-\delta d_{02}=0, d_{02}=1, d_{1}-d_{01}=-1, d_{2}-d_{1}=-1, \ldots, d_{\delta-1}-d_{\delta-2}=-1 \\
d_{\delta}-d_{\delta-1}=-1 .
\end{array}
$$

These equations determine the values of $d_{01}, d_{02}, d_{i}$ for $i \in\{1,2, \ldots, \delta\}$, that coincide with those given in the statement. The fact that $d_{i} \leq-1$ for $i \geq \delta+1$ follows from considering the remaining equations and recalling that non-free points can only appear when $j>\delta+1$.

Proposition 2.4.2. Let $Z$ be the surface given by a non-special divisorial valuation and let $S_{2}(Z)$ be the cone of $\operatorname{Pic}_{\mathbb{R}}(Z)$ defined before Lemma 2.4.1. Then the dual cone of $S_{2}^{\vee}(Z), S_{2}^{\vee}(Z)$, is generated by the following classes of divisors: $\left[F^{*}\right],\left[M^{*}\right]$, $\left\{\left[\Theta_{i}\right]\right\}_{i=1}^{\delta},\left\{\left[\Delta_{i}\right]\right\}_{i=\delta+1}^{n},\left\{\left[\Gamma_{i}\right]\right\}_{i=\delta+1}^{n}$ and $\left\{\left[\Upsilon_{i k}\right]\right\}_{i=\delta+1, k=1, \ldots, \delta-1}^{n}$, where

$$
\begin{gathered}
\Theta_{i}:=b_{i} M^{*}-\sum_{j=1}^{i} \operatorname{mult}_{p_{j}}\left(\varphi_{i}\right) E_{j}^{*}, \\
\Delta_{i}:=\left(-\delta b_{i}+c_{i}\right) F^{*}+b_{i} M^{*}-\sum_{j=1}^{i} \operatorname{mult}_{p_{j}}\left(\varphi_{i}\right) E_{j}^{*}, \\
\Gamma_{i}:=c_{i} M^{*}-\sum_{j=1}^{i}\left(\delta \operatorname{mult}_{p_{j}}\left(\varphi_{i}\right)\right) E_{j}^{*}, \text { and } \\
\Upsilon_{i k}:=\left(c_{i}-k b_{i}\right) M^{*}-\sum_{j=1}^{k}\left(c_{i}-k b_{i}\right) E_{j}^{*}-\sum_{j=k+1}^{i}\left((\delta-k) \operatorname{mult}_{p_{j}}\left(\varphi_{i}\right)\right) E_{j}^{*},
\end{gathered}
$$


and where

$$
b_{i}:=\left(\varphi_{F_{1}}, \varphi_{i}\right)_{p}, 1 \leq i \leq n, \text { and } c_{i}:=\left(\varphi_{M_{1}}, \varphi_{i}\right)_{p}, \delta+1 \leq i \leq n \text {. }
$$

Proof. Proposition 1.4.5 shows that it suffices to consider every $(n+1)$-dimensional linear subspace $H$ generated by elements of $S_{2}(Z)$ and check whether $H^{\perp}$ is generated by an element of $S_{2}^{\vee}(Z)$. We will see that these generators are those in the statement.

Denote by $\operatorname{lin}(S)$ the linear subspace generated by a set $S \subseteq \operatorname{Pic}_{\mathbb{R}}(Z)$. Then

$$
\operatorname{lin}\left(\left\{\left[\tilde{F}_{1}\right]\right\} \cup\left\{\left[E_{i}\right]\right\}_{i=1}^{n}\right)^{\perp}=\operatorname{lin}\left(\left[F^{*}\right]\right), \operatorname{lin}\left(\left\{\left[\tilde{M}_{0}\right]\right\} \cup\left\{\left[E_{i}\right]\right\}_{i=1}^{n}\right)^{\perp}=\operatorname{lin}\left(\left[M^{*}\right]\right),
$$

and $\left[F^{*}\right],\left[M^{*}\right] \in S_{2}^{\vee}(Z)$. Moreover, $\operatorname{lin}\left(\left\{\left[\tilde{M}_{1}\right]\right\} \cup\left\{\left[E_{i}\right]\right\}_{i=1}^{n}\right)^{\perp}$ is not generated by an element in $S_{2}^{\vee}(Z)$.

We have studied subspaces $H$ generated by, at least, all the classes $\left[E_{i}\right]$. Now we are going to treat the cases where a class $\left[E_{i}\right], 1 \leq i \leq n$, is not considered. Let us start with the linear space $\operatorname{lin}\left(\left\{\left[\tilde{F}_{1}\right],\left[\tilde{M}_{0}\right]\right\} \cup\left\{\left[E_{j}\right]\right\}_{1 \leq j \leq n, j \neq i}\right)$, set

$$
\left[D_{i}\right]=d_{i 01}\left[F^{*}\right]+d_{i 02}\left[M^{*}\right]+d_{i 1}\left[E_{1}^{*}\right]+\cdots+d_{i n}\left[E_{n}^{*}\right] \in \operatorname{Pic}_{\mathbb{R}}(Z)
$$

with arbitrary coefficients and impose the conditions:

$$
\left[D_{i}\right] \cdot\left[\tilde{F}_{1}\right]=0,\left[D_{i}\right] \cdot\left[\tilde{M}_{0}\right]=0,\left[D_{i}\right] \cdot\left[E_{j}\right]=0,\left[D_{i}\right] \cdot\left[\tilde{M}_{1}\right] \geq 0 \text { and }\left[D_{i}\right] \cdot\left[E_{i}\right] \geq 0 .
$$

Then we obtain the system of equalities and inequalities:

$$
\begin{gathered}
d_{i 02}+d_{i 1}=0, d_{i 01}+\delta d_{i 02}-\delta d_{i 02}=0,-d_{i j}+\sum_{p_{s} \rightarrow p_{j}} d_{i s}=0, \\
d_{i 01}+\delta d_{i 02}+\sum_{j=1}^{\min \left\{i, i_{M_{1}}\right\}} d_{i j} \geq 0 \text { and }-d_{i i}+\sum_{p_{s} \rightarrow p_{i}} d_{i s} \geq 0
\end{gathered}
$$

where $i_{M_{1}}$ is the index defined in the proof of Lemma 2.4.1. Solving the above system, we obtain $d_{i i}<0 ; d_{i j}=\sum_{p_{s} \rightarrow p_{j}} d_{i s}, 1 \leq j \leq i-1 ; d_{i j}=0, i+1 \leq j \leq n ; d_{i 01}=0$; and $d_{i 02}=-d_{i 1}$. This proves that $d_{i j}=-\operatorname{mult}_{p_{j}}\left(\varphi_{i}\right)$ holds and also

$$
\delta \operatorname{mult}_{p_{1}}\left(\varphi_{i}\right)-\sum_{j=1}^{\min \left\{i, i_{M_{1}}\right\}} \operatorname{mult}_{p_{j}}\left(\varphi_{i}\right) \geq 0
$$

by our first inequality, which shows that the classes $\left\{\left[\Theta_{i}\right]\right\}_{1 \leq i \leq \delta}$ in the statement give generators of the dual cone $S_{2}^{\vee}(Z)$.

Reasoning as above for the subspace $\operatorname{lin}\left(\left\{\left[\tilde{F}_{1}\right],\left[\tilde{M}_{1}\right]\right\} \cup\left\{\left[E_{j}\right]\right\}_{1 \leq j \leq n, j \neq i}\right)$ and with the same notation, we get the system of equalities and inequalities:

$$
\begin{gathered}
d_{i 02}+d_{i 1}=0, d_{i 01}+\delta d_{i 02}+\sum_{j=1}^{\min \left\{i, i_{M_{1}}\right\}} d_{i j}=0,-d_{i j}+\sum_{p_{s} \rightarrow p_{j}} d_{i s}=0 \\
d_{i 01}+\delta d_{i 02}-\delta d_{i 02} \geq 0 \text { and }-d_{i i}+\sum_{p_{s} \rightarrow p_{i}} d_{i s} \geq 0 .
\end{gathered}
$$


Here, the equality $d_{i j}=-\operatorname{mult}_{p_{j}}\left(\varphi_{i}\right)$ is again true and the first inequality means that

$$
\sum_{j=1}^{\min \left\{i, i_{M_{1}}\right\}} \operatorname{mult}_{p_{j}}\left(\varphi_{i}\right)-\delta \operatorname{mult}_{p_{1}}\left(\varphi_{i}\right) \geq 0
$$

must hold. As a consequence, we have proved that the classes $\left\{\left[\Delta_{i}\right]\right\}_{\delta+1 \leq i \leq n}$ in the statement give extremal rays of $S_{2}^{\vee}(Z)$.

Repeating the procedure with $\operatorname{lin}\left(\left\{\left[\tilde{M}_{0}\right],\left[\tilde{M}_{1}\right]\right\} \cup\left\{\left[E_{j}\right]\right\}_{1 \leq j \leq n, j \neq i}\right)$, the obtained system is

$$
\begin{gathered}
d_{i 01}+\delta d_{i 02}-\delta d_{i 02}=0, d_{i 01}+\delta d_{i 02}+\sum_{j=1}^{\min \left\{i, i_{M_{1}}\right\}} d_{i j}=0,-d_{i j}+\sum_{p_{s} \rightarrow p_{j}} d_{i s}=0 \\
d_{i 02}+d_{i 1} \geq 0 \text { and }-d_{i i}+\sum_{p_{s} \rightarrow p_{i}} d_{i s} \geq 0
\end{gathered}
$$

This proves, on the one hand, that $d_{i i}<0 ; d_{i j}=\sum_{p_{s} \rightarrow p_{j}} d_{i s}, 1 \leq j \leq i-1 ; d_{i j}=0$, $i+1 \leq j \leq n ; d_{i 01}=0$; and $d_{i 02}=(1 / \delta) \sum_{j=1}^{\min \left\{i, i_{M_{1}}\right\}}-d_{i j}$. On the other hand, reasoning as above, $d_{i j}=\delta \operatorname{mult}_{p_{j}}\left(\varphi_{i}\right)$ and then

$$
\sum_{j=1}^{\min \left\{i, i_{M_{1}}\right\}} \operatorname{mult}_{p_{j}}\left(\varphi_{i}\right)-\delta \operatorname{mult}_{p_{1}}\left(\varphi_{i}\right) \geq 0
$$

which shows that the set of classes $\left\{\left[\Gamma_{i}\right]\right\}_{\delta+1 \leq i \leq n}$ gives generators of $S_{2}^{\vee}(Z)$.

It only remains to consider those subspaces

$$
\operatorname{lin}\left(\left\{\left[\tilde{F}_{1}\right],\left[\tilde{M}_{0}\right],\left[\tilde{M}_{1}\right]\right\} \cup\left\{\left[E_{j}\right]\right\}_{j \in\{1,2, \ldots, n\} \backslash\{k, i\}}\right)
$$

attached to pairs of indices $k, i, 1 \leq k<i \leq n$. Lemma 2.4 .1 proves the $(n+1)$ dimensionality of these subspaces. Our computations depend on two indices $i$ and $k$. So, we write

$$
\left[D_{i k}\right]=d_{i k 01}\left[F^{*}\right]+d_{i k 02}\left[M^{*}\right]+d_{i k 1}\left[E_{1}^{*}\right]+d_{i k 2}\left[E_{2}^{*}\right]+\cdots+d_{i k n}\left[E_{n}^{*}\right] .
$$

We must impose the following conditions:

$$
\begin{array}{r}
{\left[D_{i k}\right] \cdot\left[\tilde{F}_{1}\right]=0,\left[D_{i k}\right] \cdot\left[\tilde{M}_{0}\right]=0,\left[D_{i k}\right] \cdot\left[\tilde{M}_{1}\right]=0,\left[D_{i k}\right] \cdot\left[E_{j}\right]=0,\left[D_{i k}\right] \cdot\left[E_{k}\right] \geq 0} \\
\text { and }\left[D_{i k}\right] \cdot\left[E_{i}\right] \geq 0
\end{array}
$$

which give the equivalent system

$$
\begin{gathered}
d_{i k 02}+d_{i k 1}=0, d_{i k 01}=0, d_{i k 01}+\delta d_{i k 02}+\sum_{j=1}^{\min \left\{i, i_{M_{1}}\right\}} d_{i k j}=0, \\
-d_{i k j}+\sum_{p_{s} \rightarrow p_{j}} d_{i k s}=0,-d_{i k k}+\sum_{p_{s} \rightarrow p_{k}} d_{i k s} \geq 0 \text { and }-d_{i k i}+\sum_{p_{s} \rightarrow p_{i}} d_{i k s} \geq 0 .
\end{gathered}
$$


To solve it we can assume that the inequalities are strict because, otherwise, we would obtain that $\left[D_{i k}\right]$ either vanishes or it gives the class $\left[\Theta_{\delta}\right]$. Indeed, if both inequalities are equalities, then $\left[D_{i k}\right]=0$. Otherwise, taking into account that the first $\delta+1$ points in $\mathcal{C}_{\nu}$ are free, by considering the third equality and $\delta+1 \leq i_{M_{1}}$, it holds that one of the indices $i$ or $k$ equals $\delta$. This shows that we obtain $\left[\Theta_{\delta}\right]$ as a solution.

The solutions of the system satisfy that $d_{i k i}<0 ;-d_{i k k}>-\sum_{p_{s} \rightarrow p_{k}} d_{i k s} ; d_{i k j}=$ $\sum_{p_{s} \rightarrow p_{j}} d_{i k s}, 1 \leq j \neq k \leq i-1 ; d_{i k j}=0, i+1 \leq j \leq n ; d_{i k 01}=0 ; d_{i k 02}=-d_{i k 1} ;$ and it must hold that

$$
-\delta d_{i k 1}=-\sum_{j=1}^{\min \left\{i, i_{M_{1}}\right\}} d_{i k j}
$$

by the third equation. Note that, for $k+1 \leq j \leq i, d_{i k j}=-\operatorname{mult}_{p_{j}}\left(\varphi_{i}\right)$ up to a positive factor, and also that $-d_{i k k}>-\sum_{p_{s} \rightarrow p_{k}} d_{i k s} \geq 0$.

The indices $i$ and $k$ must satisfy that $1 \leq k \leq \delta-1$ and $\delta+1 \leq i \leq n$. Indeed, with respect to $k$ and reasoning by contradiction, suppose that $k \geq \delta$. By hypothesis, $k<i, \delta+1 \leq i_{M_{1}}$, and $d_{i k j}=d_{i k \delta}$ for $1 \leq j \leq \delta-1$, because the first $\delta+1$ points in $\mathcal{C}_{\nu}$ are free, then

$$
-\sum_{j=1}^{\min \left\{i, i_{M_{1}}\right\}} d_{i k j}=-\delta d_{i k 1}-\sum_{j=\delta+1}^{\min \left\{i, i_{M_{1}}\right\}} d_{i k j}
$$

where $-\sum_{j=\delta+1}^{\min \left\{i, i_{M_{1}}\right\}} d_{i k j}>0$, which does not hold by (2.11). Notice that this equality is true by our imposed equalities. With respect to the index $i$, again reasoning by contradiction, suppose that $i \leq \delta$. As $1 \leq k \leq \delta-1$, Equality (2.11) is equivalent to

$$
-(\delta-k) d_{i k k}=-\sum_{j=k+1}^{\min \left\{i, i_{M_{1}}\right\}} d_{i k j}
$$

because $d_{i k j}=d_{i k k}$ for $1 \leq j \leq k$. This implies that $-(\delta-k) d_{i k k}=-(i-k) d_{i k k+1}$, which is a contradiction since $-d_{i k k}>-d_{i k k+1}$.

Notice that (2.11) also gives us the value of $d_{i k k}$, which can be obtained from the following chain of equalities:

$$
d_{i k k}=\delta d_{i k 1}-\sum_{j=1, j \neq k}^{\min \left\{i, i_{M_{1}}\right\}} d_{i k j}=\delta d_{i k k}-(k-1) d_{i k k}-\sum_{j=k+1}^{\min \left\{i, i_{M_{1}}\right\}} d_{i k j} .
$$

Thus, if we take $d_{i k j}=-(\delta-k) \operatorname{mult}_{p_{j}}\left(\varphi_{i}\right), k+1 \leq j \leq i$, one gets that

$$
d_{i k 1}=\cdots=d_{i k k}=\frac{-(\delta-k) \sum_{j=k+1}^{\min \left\{i, i_{M_{1}}\right\}} \operatorname{mult}_{p_{j}}\left(\varphi_{i}\right)}{(\delta-k)}=-\sum_{j=k+1}^{\min \left\{i, i_{M_{1}}\right\}} \operatorname{mult}_{p_{j}}\left(\varphi_{i}\right),
$$

and the coefficient of $\left[M^{*}\right]$ is $d_{i k 02}=-d_{i k 1}$. 
As a result, we have that $\left[D_{i k}\right]=\left[\Upsilon_{i k}\right]$, where

$$
\begin{aligned}
\Upsilon_{i k}:=\left(\sum_{j=k+1}^{\min \left\{i, i_{M_{1}}\right\}} \operatorname{mult}_{p_{j}}\left(\varphi_{i}\right)\right) M^{*} & -\sum_{j=1}^{k}\left(\sum_{s=k+1}^{\min \left\{i, i_{M_{1}}\right\}} \operatorname{mult}_{p_{s}}\left(\varphi_{i}\right)\right) E_{j}^{*} \\
& -\sum_{j=k+1}^{i}\left((\delta-k) \operatorname{mult}_{p_{j}}\left(\varphi_{i}\right)\right) E_{j}^{*},
\end{aligned}
$$

where $\delta+1 \leq i \leq n$ and $1 \leq k \leq \delta-1$. This finishes the proof.

Remark 2.4.3. From the above proof, it can be deduced that, when considering the surface $\mathbb{F}_{1}$ and a non-special valuation $\nu$, no class $\left[\Upsilon_{i k}\right]$ appears as a generator of $S_{2}^{\vee}(Z)$.

Remark 2.4.4. The divisors $\left\{\Theta_{i}\right\}_{i=1}^{\delta},\left\{\Delta_{i}\right\}_{i=\delta+1}^{n},\left\{\Gamma_{i}\right\}_{i=\delta+1}^{n}$ and $\left\{\Upsilon_{i k}\right\}_{i=\delta+1, k=1, \ldots, \delta-1}^{n}$ defined in Proposition 2.4.2 can be written as

$$
\begin{gathered}
\Theta_{i}=\Theta\left(\nu_{i}\right):=\nu_{i}\left(\varphi_{F_{1}}\right) M^{*}-\sum_{j=1}^{i} \nu_{i}\left(\mathfrak{m}_{j}\right) E_{j}^{*}, \\
\Delta_{i}=\Delta\left(\nu_{i}\right):=\left(-\delta \nu_{i}\left(\varphi_{F_{1}}\right)+\nu_{i}\left(\varphi_{M_{1}}\right)\right) F^{*}+\nu_{i}\left(\varphi_{F_{1}}\right) M^{*}-\sum_{j=1}^{i} \nu_{i}\left(\mathfrak{m}_{j}\right) E_{j}^{*}, \\
\Gamma_{i}=\Gamma\left(\nu_{i}\right):=\nu_{i}\left(\varphi_{M_{1}}\right) M^{*}-\sum_{j=1}^{i}\left(\delta \nu_{i}\left(\mathfrak{m}_{j}\right)\right) E_{j}^{*}, \text { and } \\
\Upsilon_{i k}=\Upsilon_{k}\left(\nu_{i}\right):=\left(\nu_{i}\left(\varphi_{M_{1}}\right)-k \nu_{i}\left(\varphi_{F_{1}}\right)\right) M^{*}-\sum_{j=1}^{k}\left(\nu_{i}\left(\varphi_{M_{1}}\right)-k \nu_{i}\left(\varphi_{F_{1}}\right)\right) E_{j}^{*} \\
-\sum_{j=k+1}^{i}\left((\delta-k) \nu_{i}\left(\mathfrak{m}_{j}\right)\right) E_{j}^{*},
\end{gathered}
$$

where $\nu_{i}$ is the non-special divisorial valuation defined by the exceptional divisor $E_{i}$ and $\left\{\nu_{i}\left(\mathfrak{m}_{j}\right)\right\}_{j=1}^{i}$ its sequence of values. Moreover, their self-intersections satisfy

$$
\begin{aligned}
\Theta_{i}^{2} & =\delta b_{i}^{2}-\bar{\beta}_{g+1}\left(\nu_{i}\right)=\delta \nu_{i}\left(\varphi_{F_{1}}\right)^{2}-\bar{\beta}_{g+1}\left(\nu_{i}\right) \\
\Delta_{i}^{2} & =2\left(-\delta b_{i}+c_{i}\right) b_{i}+\delta b_{i}^{2}-\bar{\beta}_{g+1}\left(\nu_{i}\right) \\
& =2 b_{i} c_{i}-\delta b_{i}^{2}-\bar{\beta}_{g+1}\left(\nu_{i}\right) \\
& =2 \nu_{i}\left(\varphi_{F_{1}}\right) \nu_{i}\left(\varphi_{M_{1}}\right)-\delta \nu_{i}\left(\varphi_{F_{1}}\right)^{2}-\bar{\beta}_{g+1}\left(\nu_{i}\right) \\
\Gamma_{i}^{2} & =\delta c_{i}^{2}-\delta^{2} \bar{\beta}_{g+1}\left(\nu_{i}\right)=\delta \nu_{i}\left(\varphi_{M_{1}}\right)^{2}-\delta^{2} \bar{\beta}_{g+1}\left(\nu_{i}\right) \\
\Upsilon_{i k}^{2} & \left.=\delta\left(c_{i}-k b_{i}\right)^{2}-k\left(c_{i}-k b_{i}\right)^{2}-(\delta-k)^{2} \sum_{j=k+1}^{n} \operatorname{mult}_{p_{j}}^{2}\left(\varphi_{i}\right)\right) \\
& =(\delta-k)\left[\left(c_{i}-k b_{i}\right)^{2}-(\delta-k)\left(\bar{\beta}_{g+1}\left(\nu_{i}\right)-k b_{i}^{2}\right)\right] \\
& =(\delta-k)\left[c_{i}^{2}-2 k c_{i} b_{i}+\delta k b_{i}^{2}-(\delta-k) \bar{\beta}_{g+1}\left(\nu_{i}\right)\right]
\end{aligned}
$$




$$
\begin{aligned}
& =(\delta-k)\left[c_{i}^{2}-k\left(2 c_{i} b_{i}-\delta b_{i}^{2}\right)-(\delta-k) \bar{\beta}_{g+1}\left(\nu_{i}\right)\right] \\
& =(\delta-k)\left[\nu_{i}\left(\varphi_{M_{1}}\right)^{2}-k\left(2 \nu_{i}\left(\varphi_{M_{1}}\right) \nu_{i}\left(\varphi_{F_{1}}\right)-\delta \nu_{i}\left(\varphi_{F_{1}}\right)^{2}\right)-(\delta-k) \bar{\beta}_{g+1}\left(\nu_{i}\right)\right],
\end{aligned}
$$

where $\bar{\beta}_{g+1}\left(\nu_{i}\right)$ is the last maximal contact value of $\nu_{i}$. Note that these divisors have a close behaviour to the divisors $\left\{\Lambda_{i}\right\}_{i=1}^{n}$ defined in Proposition 2.3.1,

The before mentioned divisors introduced in Proposition 2.4.2 are essential to characterize the cone of curves $\mathrm{NE}(Z)$ of a surface $Z$ defined by a non-special divisorial valuation of a Hirzebruch surface. Some of their properties are stated in the forthcoming lemma.

Lemma 2.4.5. Let $Z$ (respectively, $\nu_{n}$ ) be a rational surface (respectively, a valuation) as in Proposition 2.4.2. Consider the set of divisors there defined. Then $\Delta_{\delta+1}^{2}>0, \Gamma_{\delta+1}^{2}>0$ and $\Upsilon_{\delta+1 k}^{2}>0$ for all $k \in\{1,2, \ldots, \delta-1\}$. In addition, for any index $i \in\{\delta+2, \delta+3, \ldots, n\}$ such that $\Delta_{i}^{2} \geq 0$ (respectively, $\Gamma_{i}^{2} \geq 0, \Upsilon_{i k}^{2} \geq 0$ ), the following properties are satisfied:

(a) If $p_{i}$ is a satellite point of the configuration $\mathcal{C}_{\nu_{n}}$ that $\nu_{n}$ defines, it holds $\Delta_{i}^{2}>0$ (respectively, $\Gamma_{i}^{2}>0, \Upsilon_{i k}^{2}>0$ ).

(b) $\Delta_{i-1}^{2} \geq 0$ (respectively, $\Gamma_{i-1}^{2} \geq 0, \Upsilon_{i-1 k}^{2} \geq 0$ ) and, moreover, if $\Delta_{i-1}^{2}=0$ (respectively, $\Gamma_{i-1}^{2}=0, \Upsilon_{i-1 k}^{2}=0$ ) then $p_{i}$ is a satellite point and $p_{i-1}$ is free.

Proof. To prove our first assertion, it suffices to notice that the following three equalities hold:

$$
\begin{aligned}
\Delta_{\delta+1}^{2} & =2+\delta-(\delta+1)=1>0, \\
\Gamma_{\delta+1}^{2} & =\delta(\delta+1)^{2}-\delta^{2}(\delta+1)=\delta(\delta+1)(\delta+1-\delta)=\delta(\delta+1)>0, \\
\Upsilon_{\delta+1 k}^{2} & =\delta(\delta+1-k)^{2}-k(\delta+1-k)^{2}-(\delta+1-k)(\delta-k)^{2} \\
& =(\delta-k)(\delta+1-k)^{2}-(\delta-k)^{2}(\delta+1-k) \\
& =(\delta-k)(\delta+1-k)[\delta-k+1-(\delta-k)] \\
& =(\delta-k)(\delta+1-k)>0 .
\end{aligned}
$$

Finally we remark that (a) and (b) can be proved reasoning as in the proof of Lemma 2.3.6. Indeed, recalling that $g+2$ is the cardinality of the set of maximal contact values of $\nu_{n}$, the case $g=1$ follows as in that proof, and, when $g>1$, with notations as in that lemma and in Proposition 2.4.2, it suffices to consider the following equalities and to reason again as we did in the mentioned Lemma 2.3.6.

$$
\begin{aligned}
\Delta_{n}^{2} & =2 b_{n} c_{n}-\delta b_{n}^{2}-\bar{\beta}_{g+1}\left(\nu_{n}\right)=e_{g-1}\left(\nu_{n}\right)\left[\frac{2 b_{n} c_{n}-\delta b_{n}^{2}}{e_{g-1}\left(\nu_{n}\right)}-\bar{\beta}_{g}\left(\nu_{n}\right)\right], \\
\Gamma_{n}^{2} & =c_{n}^{2} \delta-\delta^{2} \bar{\beta}_{g+1}\left(\nu_{n}\right), \quad \text { and } \\
\Upsilon_{n k}^{2} & =(\delta-k)\left[c_{n}^{2}-k\left(2 c_{n} b_{n}-\delta b_{n}^{2}\right)-(\delta-k) \bar{\beta}_{g+1}\left(\nu_{n}\right)\right] .
\end{aligned}
$$


Remark 2.4.6. The non-negativity of the self-intersection of the divisors introduced in Proposition 2.4.2 can be numerically determined. By Remark 2.4.4, it holds that $\Theta_{i}^{2}=\delta-i \geq 0,1 \leq i \leq \delta$, since the points $p_{1}, p_{2} \ldots, p_{\delta}$ are free. For the remaining divisors, these conditions follow from Remark 2.4.4 and Lemma 2.4.5.

Indeed, the inequality $\Delta_{n}^{2} \geq 0$ (or, equivalently, $2 b_{n} c_{n}-\delta b_{n}^{2} \geq \bar{\beta}_{g+1}\left(\nu_{n}\right)$ ) implies that $\Delta_{i}^{2} \geq 0$, that is, $2 b_{i} c_{i}-\delta b_{i}^{2} \geq \bar{\beta}_{g+1}\left(\nu_{i}\right)$ for $1 \leq i \leq n$, where $\nu_{i}$ is the divisorial valuation defined as in Remark 2.4.4. Likewise, when $\Gamma_{n}^{2} \geq 0$, it holds that $\Gamma_{i}^{2} \geq 0$, or equivalently that $c_{i}^{2} \geq \delta \bar{\beta}_{g+1}\left(\nu_{i}\right)$, for $1 \leq i \leq n$.

To finish, given a positive integer $k, 1 \leq k \leq \delta-1$, the inequality

$$
c_{n}^{2}-k\left(2 c_{n} b_{n}-\delta b_{n}^{2}\right) \geq(\delta-k) \bar{\beta}_{g+1}\left(\nu_{n}\right),
$$

allows us to conclude that $\Upsilon_{i k}^{2} \geq 0$, for $1 \leq i \leq n$, which numerically can be expressed as $c_{i}^{2}-k\left(2 c_{i} b_{i}-\delta b_{i}^{2}\right) \geq(\delta-k) \bar{\beta}_{g+1}\left(\nu_{i}\right)$.

The next lemma will be useful for proving the main result in this section.

Lemma 2.4.7. Let $\nu_{n}$ be a non-special divisorial valuation of a Hirzebruch surface and $Z$ the surface that it defines. Consider the divisors $\Delta_{i}, \Gamma_{i}$ and $\Upsilon_{i k}, \delta+1 \leq i \leq n$; $1 \leq k \leq \delta-1$, given in Proposition 2.4.2. Then, for each index $i, \Delta_{i}^{2} \geq 0$ implies $\Gamma_{i}^{2} \geq 0$ and $\Upsilon_{i k}^{2} \geq 0$ for all $k \in\{1,2, \ldots, \delta-1\}$.

Proof. Our proof is consequence of the following two properties:

Property 1: If the self-intersections of the divisors $\Delta_{i}$ and $\Upsilon_{i \delta-1}$ are non-negative, then the same property holds for the divisors $\Gamma_{i}$ and $\Upsilon_{i k}, 1 \leq k \leq \delta-1$.

Property 2: If the self-intersection of the divisor $\Delta_{i}$ is non-negative, so is the self-intersection of $\Upsilon_{i \delta-1}$.

For proving Property 1, our hypotheses are, by Remark 2.4.6,

$$
\begin{gathered}
\bar{\beta}_{g+1}\left(\nu_{i}\right) \leq 2 c_{i} b_{i}-\delta b_{i}^{2} \quad \text { and } \\
(\delta-1)\left(2 c_{i} b_{i}-\delta b_{i}^{2}-\bar{\beta}_{g+1}\left(\nu_{i}\right)\right) \leq c_{i}^{2}-\delta \bar{\beta}_{g+1}\left(\nu_{i}\right) .
\end{gathered}
$$

The inequality in (2.14) and the following one

$$
\bar{\beta}_{g+1}\left(\nu_{i}\right) \leq c_{i}^{2}-(\delta-1)\left(2 c_{i} b_{i}-\delta b_{i}^{2}\right)
$$

are equivalent. From the last inequality and the one in (2.13), we get that $c_{i}^{2} \geq$ $\delta \bar{\beta}_{g+1}\left(\nu_{i}\right)$ and then $\Gamma_{i}^{2} \geq 0$. Finally, $\Upsilon_{i k}^{2} \geq 0,1 \leq k \leq \delta-1$, if and only if the inequality

$$
k\left(2 c_{i} b_{i}-\delta b_{i}^{2}-\bar{\beta}_{g+1}\left(\nu_{i}\right)\right) \leq c_{i}^{2}-\delta \bar{\beta}_{g+1}\left(\nu_{i}\right)
$$

holds, fact that follows straightforwardly from (2.13) and (2.14).

To conclude we prove Property 2. It suffices to check that the following inequalities

$$
\bar{\beta}_{g+1}\left(\nu_{i}\right) \leq 2 c_{i} b_{i}-\delta b_{i}^{2}<c_{i}^{2}-(\delta-1)\left(2 c_{i} b_{i}-\delta b_{i}^{2}\right)
$$


are true. In fact, the first inequality comes from our hypothesis $\Delta_{i}^{2} \geq 0$ and the inequality given by the first and the last sides in (2.15) allows us to show $\Upsilon_{i \delta-1}^{2} \geq 0$. To prove the second inequality in (2.15), set $c_{i}=x$ and $b_{i}=b$ for simplicity. We are considering non-special valuations, which means that $x>\delta b$. In our new notation we want to prove that

$$
2 b x-\delta b^{2}<x^{2}-(\delta-1)\left(2 b x-\delta b^{2}\right) .
$$

This inequality is equivalent to

$$
0<x^{2}-(2 b \delta) x+\delta^{2} b^{2}
$$

and it holds for all $x \neq \delta b$ since the point $(\delta b, 0)$ is the vertex of the parabola given by the right-hand side of the inequality.

In the same way that Theorem 2.3.7 gives equivalent conditions for the nonpositivity of special divisorial valuations, the forthcoming theorem provides two interesting geometrical conditions characterizing the non-positivity at infinity of nonspecial divisorial valuations. It also includes a numerical and local expression that can be easily checked.

Theorem 2.4.8. Let $\nu_{n}$ be a non-special divisorial valuation of the function field of $\mathbb{F}_{\delta}$ centered at $\mathcal{O}_{\mathbb{F}_{\delta}, p}$ and $\mathcal{C}_{\nu_{n}}=\left\{p_{i}\right\}_{i=1}^{n}$ its configuration of infinitely near points. Let $Z$ be the surface that $\nu_{n}$ defines and consider the divisor $\Delta_{n}$ on $Z$ defined in Proposition 2.4.2. Then the following conditions are equivalent:

(a) The valuation $\nu_{n}$ is non-positive at infinity.

(b) The divisor $\Delta_{n}$ is nef.

(c) It holds the following inequality: $2 c_{n} b_{n}-\delta b_{n}^{2} \geq \bar{\beta}_{g+1}\left(\nu_{n}\right)$.

(d) The cone of curves of $Z$ is generated by $\left[\tilde{F}_{1}\right],\left[\tilde{M}_{0}\right],\left[\tilde{M}_{1}\right],\left[E_{1}\right],\left[E_{2}\right], \ldots,\left[E_{n}\right]$.

Proof. Our proof uses a close reasoning to that of Theorem 2.3.7. Keeping the notation as in that theorem, we are going to give a sketch of the proof emphasizing only the main differences.

To prove that (a) can be deduced from (b), we can suppose that $p$ is a general point of $\mathbb{F}_{\delta}$ with coordinates $(0: 1 ; 0,1)$. Consider local coordinates $\{x, y\}=$ $\left\{\frac{X_{1}}{X_{0}}, \frac{X_{0}^{\delta} Y_{1}}{Y_{0}}\right\}$ in the affine open set $U_{00}$ and $\{u, v\}=\left\{\frac{X_{0}}{X_{1}}, \frac{Y_{0}}{X_{1}^{\delta} Y_{1}}\right\}$ in $U_{11}$. Notice that, with our notation, $F_{1}$ and $M_{1}$ are defined by the equations $X_{0}=0$ and $Y_{0}=0$, $p \in U_{11}$, and $F_{1}$ and $M_{1}$ have local equations $u=0$ and $v=0$, respectively.

If now $\mathcal{S}$ denotes the set of non-constant polynomials in $\mathcal{O}_{\mathbb{F}_{\delta}}\left(U_{00}\right)$ (up to multiplication by a nonzero element of $k$ ) such that neither $x$ nor $y$ divide them, $f \in \mathcal{S}$ satisfies

$$
f(x, y)=f\left(1 / u, u^{\delta} / v\right)=\frac{h_{f}(u, v)}{u^{\operatorname{deg}_{1}\left(h_{f}\right)} v^{\operatorname{deg}_{2}\left(h_{f}\right)}},
$$


where $h_{f}(u, v) \in \mathcal{O}_{\mathbb{F}_{\delta}}\left(U_{11}\right)$.

The bi-homogeneous polynomial $X_{1}^{\operatorname{deg}_{1}\left(h_{f}\right)+\delta \operatorname{deg}_{2}\left(h_{f}\right)} Y_{1}^{\operatorname{deg}_{2}\left(h_{f}\right)} h_{f}\left(\frac{X_{0}}{X_{1}}, \frac{Y_{0}}{X_{1}^{\delta} Y_{1}}\right)$ defines a curve $C_{f}$ on $\mathbb{F}_{\delta}$ of degree $\left(\operatorname{deg}_{1}\left(h_{f}\right), \operatorname{deg}_{2}\left(h_{f}\right)\right)$ and $f \mapsto C_{f}$ is a one-to-one correspondence between $\mathcal{S}$ and the set of curves on $\mathbb{F}_{\delta}$ containing no curve $F_{1}, F^{\prime}, M_{0}, M_{1}$ as a component, where $F^{\prime}$ and $M_{0}$ are defined by the equations $X_{0}=0$ and $Y_{1}=0$. Then $\Delta_{n} \cdot C_{f}=-\nu(f)$ and by $(\mathrm{b}),-\nu(f) \geq 0$. The case when $f \in \mathcal{O}_{\mathbb{F}_{\delta}}\left(U_{00}\right)$ and $x$ or $y$ or both are factors of $f$ follows as in Theorem 2.3.7 and (a) is proved.

A proof of the fact that (a) implies (b), (b) implies (c) and (d) implies (b) can be done as in Theorem 2.3.7.

To see that (c) implies (d), it suffices to notice that, by Lemmas 2.4.5 and 2.4.7,

$$
S_{2}^{\vee}(Z) \subseteq\left\{[D] \in \operatorname{Pic}_{\mathbb{R}}(Z) \mid[D]^{2} \geq 0 \text { and }[H] \cdot[D] \geq 0\right\}=: A(Z),
$$

where $S_{2}^{\vee}(Z)$ is the dual cone defined in Proposition 2.4.2 and $H$ an ample divisor on $Z$. Finally, the fact

$$
S_{2}^{\vee}(Z) \subseteq A(Z) \subseteq\left(S_{2}^{\vee}(Z)\right)^{\vee}=S_{2}(Z)
$$

and a reasoning as in Theorem 2.3.7 completes our proof.

Remark 2.4.9. The extremal rays of the nef cone $\operatorname{Nef}(Z)$ of $Z$ are the elements of the set $\left\{\left[F^{*}\right],\left[M^{*}\right]\right\} \cup\left\{\left[\Theta_{i}\right]\right\}_{i=1}^{\delta} \cup\left\{\left[\Delta_{i}\right]\right\}_{i=\delta+1}^{n} \cup\left\{\left[\Gamma_{i}\right]\right\}_{i=\delta+1}^{n} \cup\left\{\left[\Upsilon_{i \ell}\right]\right\}_{\delta+1 \leq i \leq n}^{1 \leq k \leq \delta-1}$, by Proposition 2.4.2 and Theorem 2.4.8.

As in the special case, one has the following two corollaries as a consequence of the above theorem.

Corollary 2.4.10. Let $\nu_{n}$ be a non-positive at infinity non-special divisorial valuation of $\mathbb{F}_{\delta}$. Consider the divisorial valuations $\nu_{i}$ defined by the divisors $E_{i}$ associated to the simple sequence of point blowups that $\nu_{n}$ defines. Then the valuations $\nu_{i}$, $\delta+1 \leq i \leq n-1$, are non-positive at infinity (non-special of $\mathbb{F}_{\delta}$ ).

Proof. This result follows from the same reasoning that Corollary 2.3.10 using Theorem 2.4.8, Lemmas 2.4.5 and 2.4.7 and Remark 2.4.6.

Corollary 2.4.11. Let $Z$ be a surface as in Theorem 2.4.8 defined by a non-positive at infinity non-special valuation. Then all the divisors $\Theta_{i}, i=1,2, \ldots, \delta ; \Delta_{i}, \Gamma_{i}$ and $\Upsilon_{i k}, i=\delta+1, \delta+2, \ldots, n$ and $k=1,2, \ldots, \delta-1$, defined in Proposition 2.4.2, are effective. In particular, it holds that

$$
\begin{gathered}
\Theta_{i}=\left(\Theta_{i} \cdot F^{*}\right) \tilde{M}_{1}+\sum_{j=1}^{n}\left(\Theta_{i} \cdot \Delta_{j}\right) E_{j}, \\
\Delta_{i}=\left(\Delta_{i} \cdot M_{0}^{*}\right) \tilde{F}_{1}+\left(\Delta_{i} \cdot F^{*}\right) \tilde{M}_{1}+\sum_{j=1}^{n}\left(\Delta_{i} \cdot \Delta_{j}\right) E_{j}, \\
\Gamma_{i}=\left(\Gamma_{i} \cdot F^{*}\right) \tilde{M}_{1}+\sum_{j=1}^{n}\left(\Gamma_{i} \cdot \Delta_{j}\right) E_{j},
\end{gathered}
$$




$$
\Upsilon_{i k}=\left(\Upsilon_{i k} \cdot F^{*}\right) \tilde{M}_{1}+\sum_{j=1}^{n}\left(\Upsilon_{i k} \cdot \Delta_{j}\right) E_{j} .
$$

Proof. Notice that the set $\left\{F^{*}, M^{*}\right\} \cup\left\{\Delta_{i}\right\}_{i=1}^{n}$ is the dual basis of the basis $\left\{\tilde{F}_{1}, \tilde{M}_{1}\right\} \cup$ $\left\{E_{i}\right\}_{i=1}^{n}$ of $\operatorname{Pic}_{\mathbb{R}}(Z)$ by Proposition 2.4.2, and each divisor of the first set is nef and has non-negative self-intersection by Corollary 2.4.10 and then its class belongs to $A(Z) \subset \overline{\mathrm{NE}}(Z)$. Finally, arguing as in Corollary 2.3.11, the result is proved.

As in the non-positive at infinity special case, we can set a sequence of nonpositive at infinity non-special divisorial valuations of $\mathbb{F}_{\delta}$ which approaches a nonpositive at infinity non-special irrational valuation. The proof follows from the arguments used to prove Corollary 2.3.12.

Corollary 2.4.12. Let $\nu_{n}$ be a non-positive at infinity non-special divisorial valuation of $\mathbb{F}_{\delta}$ and $\mathcal{C}_{\nu_{n}}=\left\{p_{i}\right\}_{i=1}^{n}$ its configuration of infinitely near points. Set a non-special divisorial valuation $\nu_{m}$ such that its configuration $\mathcal{C}_{\nu_{m}}=\left\{q_{i}\right\}_{i=1}^{m}, n<m$, satisfies that $\mathcal{C}_{\nu_{n}} \subset \mathcal{C}_{\nu_{m}}$ and the points $q_{i}, n+1 \leq i \leq m$, are satellite. Then, the valuation $\nu_{m}$ is non-positive at infinity.

Example 2.4.13. Let $\nu$ be a non-special divisorial valuation of the Hirzebruch surface $\mathbb{F}_{2}$ whose sequence of maximal contact values is $\{15,51,262,786\}$. Set $\mathcal{C}_{\nu}=$ $\left\{p_{i}\right\}_{i=1}^{12}$ the configuration of infinitely near points of $\nu$ and $\Gamma_{n}$ (Figure 2.2) its dual graph. Denote by $F_{1}$ the fiber of $\mathbb{F}_{2}$ that goes through $p_{1}$, by $M_{0}$ the special section

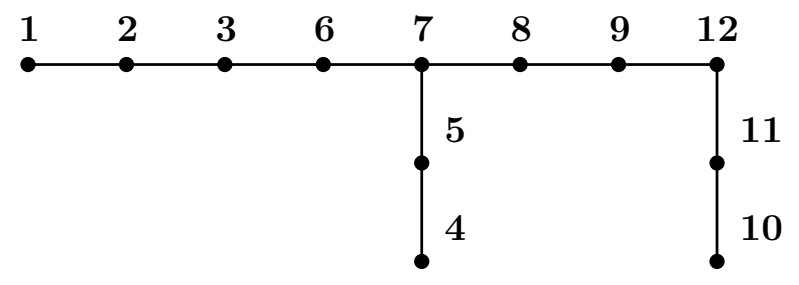

Figure 2.2: Dual graph of $\nu$ in Example 2.4.13.

and by $M_{1}$ the integral curve that is linearly equivalent to $M$ whose its strict transform pass through $p_{1}, p_{2}$ and $p_{3}$. Then $b_{12}=15, c_{12}=45$ and $\bar{\beta}_{g+1}(\nu)=786$, and so, Theorem 2.4.8 (c) is satisfied. Therefore, the cone of curves of the surface $Z$ defined by $\nu$ is generated by $\left\{\left[\tilde{F}_{1}\right],\left[\tilde{M}_{0}\right],\left[\tilde{M}_{1}\right]\right\} \cup\left\{E_{i}\right\}_{i=1}^{12}$ and the divisors $\Delta_{i}, 1 \leq i \leq 12$, defined in Proposition 2.4.2 are nef.

We conclude the section stating a result that provides two equivalent conditions characterizing the negativity at infinity of non-special divisorial valuations. It can be proved as we did in Theorem 2.3.14.

Theorem 2.4.14. Keeping the same assumptions and notations as in Theorem 2.4.8, the following conditions are equivalent: 
(a) The valuation $\nu_{n}$ is negative at infinity.

(b) It holds that either $2 c_{n} b_{n}-b_{n}^{2} \delta>\bar{\beta}_{g+1}\left(\nu_{n}\right)$, or $2 c_{n} b_{n}-b_{n}^{2} \delta=\bar{\beta}_{g+1}\left(\nu_{n}\right)$ and the Iitaka dimension of the divisor $\Delta_{n}$ vanishes.

(c) The inequality $\Delta_{n} \cdot \tilde{C}>0$ is satisfied for the strict transform on $Z$, $\tilde{C}$, of any curve $C$ on $\mathbb{F}_{\delta}, C \neq F_{1}, M_{1}$.

\subsection{Discrete equivalence of NPI valuations}

In this section we study the dual graphs of NPI divisorial and irrational valuations of the projective plane and the Hirzebruch surfaces. Moreover, we will provide an algorithm to compute explicitly those dual graphs which admit NPI valuations. We keep the notation of the above sections. As at the beginning of the chapter, $Z_{0}$ denotes either the projective plane $\mathbb{P}^{2}$ or a Hirzebruch surface $\mathbb{F}_{\delta}, \delta \geq 0$.

As we have seen in Subsection 1.3.1, the sequence of Puiseux exponents of a plane valuation and its dual graph are very close data. The mentioned sequence allows us to work easily with the dual graph. For this reason we introduce the following definition.

Definition 2.5.1. Let $\nu$ be a divisorial or irrational valuation of $Z_{0}$. Denote by $\left\{\beta_{j}^{\prime}(\nu)\right\}_{j=0}^{g+1}$ the sequence of Puiseux exponents of $\nu$. We call discrete class of $\nu$ to the tuple

$$
\mathbf{d}(\nu)=\left(g, \beta_{0}^{\prime}(\nu), \beta_{1}^{\prime}(\nu), \ldots, \beta_{g+1}^{\prime}(\nu)\right) .
$$

Two valuations as before which have the same discrete class are named discretely equivalent.

The next result is a consequence of the definition of sequence of Puiseux exponents of a plane valuation.

Proposition 2.5.2. Let $\nu, \nu^{\prime}$ be two divisorial or irrational valuations. Then the dual graphs of $\nu$ and $\nu^{\prime}$ coincide if and only if $\nu$ and $\nu^{\prime}$ have the same discrete class.

Before stating a characterization of the discrete classes of NPI valuations, we present a lemma explaining how to get the last contact value of a plane valuation from its sequence of Puiseux exponents.

Lemma 2.5.3. Let $\nu$ be a divisorial or irrational valuation of $Z_{0}$ and $\mathbf{d}(\nu)=$ $\left(g, \beta_{0}^{\prime}(\nu), \beta_{1}^{\prime}(\nu), \ldots, \beta_{g+1}^{\prime}(\nu)\right)$ its discrete class. Then the last maximal contact value of $\nu$ satisfies

$$
\bar{\beta}_{g+1}(\nu)=\sum_{j=0}^{g} e_{j}(\nu)^{2}\left(\beta_{j+1}^{\prime}(\nu)-1\right)+e_{0}(\nu)^{2} \beta_{0}^{\prime}(\nu)
$$


Proof. By Equality (1.5) and since $e_{g}(\nu)=\operatorname{gcd}\left(\bar{\beta}_{0}(\nu), \bar{\beta}_{1}(\nu), \ldots, \bar{\beta}_{g}(\nu)\right)=1$, it holds that

$$
\bar{\beta}_{g+1}(\nu)=e_{g}(\nu)^{2}\left(\beta_{g+1}^{\prime}(\nu)-1\right)+e_{g-1}(\nu) \bar{\beta}_{g}(\nu) .
$$

Now, again by Condition (1.5), one has

$$
e_{j}(\nu) \bar{\beta}_{j+1}(\nu)=e_{j}(\nu)^{2}\left(\beta_{j+1}^{\prime}(\nu)-1\right)+e_{j-1}(\nu) \bar{\beta}_{j}(\nu),
$$

for $1 \leq j \leq g-1$. This concludes the proof after noticing that $e_{0}(\nu)=\bar{\beta}_{0}(\nu)$.

Recall that the coordinates of a discrete class $\mathbf{d}=\left(g, \beta_{0}^{\prime}, \beta_{1}^{\prime}, \ldots, \beta_{g+1}^{\prime}\right)$ of a divisorial valuation satisfy that $g$ and $\beta_{g+1}^{\prime}$ are non-negative integers, $\beta_{0}^{\prime}=1$ and (when $g>0$ ) $\beta_{j}^{\prime}=q_{j} / n_{j} \in \mathbb{Q}_{>0} \backslash \mathbb{Z}$, where $\operatorname{gcd}\left(q_{j}, n_{j}\right)=1$. Moreover, for each $\mathbf{d}$ we define $e_{g}=1, e_{j}=\prod_{k=j+1}^{g} n_{k}, 0 \leq j<g$, and $\tau_{j}=e_{j} / e_{0}$, for $0 \leq j \leq g$. When we consider discrete classes of irrational valuations, their coordinates are defined similarly but $\beta_{g+1}^{\prime} \in \mathbb{R}_{>0} \backslash \mathbb{Q}$. We gave more information about these values in Subsection 1.3.1.

In what follows, we denote by $\mathbb{D}$ the set of tuples $\mathbf{d}$ as before.

Theorem 2.5.4. Set a class $\mathbf{d} \in \mathbb{D}$. Then

(a) There exists an NPI divisorial or irrational valuation of $\mathbb{P}^{2}$ with discrete class $\mathbf{d}$ if, and only if, the following inequality holds:

$$
\beta_{1}^{\prime 2} \geq \sum_{j=0}^{g} \tau_{j}^{2}\left(\beta_{j+1}^{\prime}-1\right)+\beta_{0}^{\prime} .
$$

(b) There exists an NPI special divisorial or irrational valuation of $\mathbb{F}_{\delta}$ with discrete class $\boldsymbol{d}$ if, and only if, $\delta$ is a non-negative integer and the following inequality

$$
\beta_{1}^{\prime}\left(\delta \beta_{1}^{\prime}+2\right) \geq \sum_{j=0}^{g} \tau_{j}^{2}\left(\beta_{j+1}^{\prime}-1\right)+\beta_{0}^{\prime}
$$

holds.

(c) There exists an NPI non-special divisorial or irrational valuation of $\mathbb{F}_{\delta}$ with discrete class $\boldsymbol{d}$ if, and only if, it holds that

$$
2 \beta_{1}^{\prime}-\delta \geq \sum_{j=0}^{g} \tau_{j}^{2}\left(\beta_{j+1}^{\prime}-1\right)+\beta_{0}^{\prime} .
$$

Proof. We only need to show the result for the set of discrete classes of divisorial valuations because the irrational case follows from the divisorial one and Theorem 1.3.2(c).

Let $\mathbf{d}=\left(g, \beta_{0}^{\prime}, \beta_{1}^{\prime}, \ldots, \beta_{g+1}^{\prime}\right)$ be a discrete class where $\beta_{g+1}^{\prime}$ is a positive integer. Set $\bar{\beta}_{0}, \bar{\beta}_{1}$ and $\bar{\beta}_{g+1}$ the values which can be computed from the components of $\mathbf{d}$ following the formulas given in Equality (1.5) and Lemma 2.5.3. 
We first prove (a). By Lemma 2.5.3 and the equality $\beta_{1}^{\prime}=\bar{\beta}_{1} / \bar{\beta}_{0}$, it suffices to show that $\mathbf{d}$ is the discrete class of an NPI divisorial valuation of $\mathbb{P}^{2}$ if and only if $\bar{\beta}_{1}^{2} \geq \bar{\beta}_{g+1}$. Assume first that $\nu_{n}$ is an NPI divisorial valuation of $\mathbb{P}^{2}$ whose discrete class is d. Following Theorem 2.1.2, one has

$$
\left(\varphi_{L}, \varphi_{n}\right)_{p}^{2}=\nu_{n}\left(\varphi_{L}\right)^{2} \geq \bar{\beta}_{g+1}
$$

Now, if the strict transform of $L$ goes through all initial free points in $\mathcal{C}_{\nu_{n}}$, then the equality $\nu_{n}\left(\varphi_{L}\right)=\bar{\beta}_{1}$ holds. Otherwise, $\nu_{n}\left(\varphi_{L}\right)=s_{L} \bar{\beta}_{0}$, where $1 \leq s_{L} \leq\left\lfloor\bar{\beta}_{1} / \bar{\beta}_{0}\right\rfloor$. As a result one gets that $\bar{\beta}_{1}^{2} \geq \bar{\beta}_{g+1}$ and this implication is proved. Conversely, if we set a discrete class $\mathbf{d} \in \mathbb{D}$ (where $\beta_{g+1}^{\prime} \in \mathbb{Z}_{>0}$ ) such that $\bar{\beta}_{1}^{2} \geq \bar{\beta}_{g+1}$, it suffices to take a divisorial valuation $\nu$ of $\mathbb{P}^{2}$ whose discrete class is $\mathbf{d}$ and whose first free points in $\mathcal{C}_{\nu}$ are determined the projective line $L$. Consequently the equality $\nu\left(\varphi_{L}\right)=\bar{\beta}_{1}$ is satisfied and the proof is over by Theorem 2.1.2.

As in the proof of (a), to show the equivalence claimed in (b) we are going to use the fact that the inequality given there is equivalent to the following one:

$$
2 \bar{\beta}_{1} \bar{\beta}_{0}+\delta \bar{\beta}_{1}^{2} \geq \bar{\beta}_{g+1} .
$$

Now suppose that $\mathbf{d}$ is the discrete class of a special divisorial valuation $\nu_{n}$ of $\mathbb{F}_{\delta}$. By Theorem 2.3.7, the inequality

$$
2\left(\varphi_{F_{1}}, \varphi_{n}\right)_{p}\left(\varphi_{M_{0}}, \varphi_{n}\right)_{p}+\delta\left(\varphi_{F_{1}}, \varphi_{n}\right)_{p}^{2}=2 \nu_{n}\left(\varphi_{F_{1}}\right) \nu_{n}\left(\varphi_{M_{0}}\right)+\delta \nu_{n}\left(\varphi_{F_{1}}\right)^{2} \geq \bar{\beta}_{g+1}
$$

holds. Now $\nu_{n}\left(\varphi_{F_{1}}\right)$ is equal either to $\bar{\beta}_{1}$ if $\tilde{F}_{1}$ goes through all initial free points in $\mathcal{C}_{\nu_{n}}$ or to $s_{F_{1}} \bar{\beta}_{0}$, where $1 \leq s_{F_{1}} \leq\left\lfloor\bar{\beta}_{1} / \bar{\beta}_{0}\right\rfloor$, otherwise. The section $M_{0}$ has a behaviour like $F_{1}$ and then $\nu_{n}\left(\varphi_{M_{0}}\right)$ equals either $\bar{\beta}_{1}$ or $s_{M_{0}} \bar{\beta}_{0}$, where $0 \leq s_{M_{0}} \leq\left\lfloor\bar{\beta}_{1} / \bar{\beta}_{0}\right\rfloor$. This proves that $2 \bar{\beta}_{1} \bar{\beta}_{0}+\delta \bar{\beta}_{1}^{2} \geq \bar{\beta}_{g+1}$ since $F_{1}$ and $M_{0}$ pass both through $p$ but at most one of their strict transforms goes through $p_{2}$. Conversely, set a class $\mathbf{d} \in \mathbb{D}$ (where $\beta_{g+1}^{\prime} \in \mathbb{Z}_{>0}$ ) such that the inequality $2 \bar{\beta}_{1} \bar{\beta}_{0}+\delta \bar{\beta}_{1}^{2} \geq \bar{\beta}_{g+1}$ is satisfied, then taking the special divisorial valuation $\nu$ of $\mathbb{F}_{\delta}$ with discrete class $\mathbf{d}$ whose first free points in $\mathcal{C}_{\nu}$ coincide with those through which $\tilde{F}_{1}$ goes, by Theorem 2.3.7, one obtains an NPI special divisorial valuation of $\mathbb{F}_{\delta}$ with discrete class $\mathbf{d}$.

Finally arguing as before one can give a proof of (c) which is supported in the next two facts. First, by Theorem 2.4.8 the inequality in the statement is equivalent to the following one

$$
2\left(\varphi_{F_{1}}, \varphi_{n}\right)_{p}\left(\varphi_{M_{1}}, \varphi_{n}\right)_{p}-\delta\left(\varphi_{F_{1}}, \varphi_{n}\right)_{p}^{2}=2 \nu_{n}\left(\varphi_{M_{1}}\right) \nu_{n}\left(\varphi_{F_{1}}\right)-\delta \nu_{n}\left(\varphi_{F_{1}}\right)^{2} \geq \bar{\beta}_{g+1} .
$$

Second, the fiber $F_{1}$ and the section $M_{1}$ satisfy that $\nu_{n}\left(\varphi_{F_{1}}\right)=\bar{\beta}_{0}$ and $\nu_{n}\left(\varphi_{M_{1}}\right)$ equals either $\bar{\beta}_{1}$ or $s_{M_{1}} \bar{\beta}_{0}$, where $\delta+1 \leq s_{M_{1}} \leq\left\lfloor\bar{\beta}_{1} / \bar{\beta}_{0}\right\rfloor$. This completes the proof.

The following result explains the relation among the dual graphs of the different types of NPI valuations using their discrete classes and the above theorem. Before 
stating the result, denote by $\mathbf{D}_{\mathbb{P}^{2}}$ (respectively, $\mathbf{D}_{\mathbb{F}_{\delta}}^{1}, \mathbf{D}_{\mathbb{F}_{\delta}}^{2}$ ) the set of discrete classes in $\mathbb{D}$ which represent to some (respectively, special, non-special) NPI divisorial or irrational valuation of $\mathbb{P}^{2}$ (respectively, $\mathbb{F}_{\delta}$ ).

Theorem 2.5.5. Let $\mathbf{D}_{\mathbb{P}^{2}}, \mathbf{D}_{\mathbb{F}_{\delta}}^{1}, \mathbf{D}_{\mathbb{F}_{\delta}}^{2}$ be the sets introduced before. Write $\mathbf{D}_{\mathbb{P}^{2}}^{\leq 2}$ (respectively, $\mathbf{D}_{\mathbb{P}^{2}}^{\geq 2}$ ) the set of discrete classes in $\mathbf{D}_{\mathbb{P}^{2}}$ whose coordinate $\beta_{1}^{\prime}$ satisfies $\beta_{1}^{\prime} \leq 2$ (respectively, $\beta_{1}^{\prime} \geq 2$ ). Set similarly $\mathbf{D}_{\mathbb{F}_{0}}^{1, \leq 2}$ and $\mathbf{D}_{\mathbb{F}_{0}}^{1, \geq 2}$. Then

(a) $\mathbf{D}_{\mathbb{P}^{2}} \subseteq \mathbf{D}_{\mathbb{F}_{\delta}}^{1}$ for all $\delta>0$.

(b) $\mathbf{D}_{\mathbb{P}^{2}}^{\leq 2} \subseteq \mathbf{D}_{\mathbb{F}_{0}}^{1, \leq 2}$ and $\mathbf{D}_{\mathbb{F}_{0}}^{1, \geq 2} \subseteq \mathbf{D}_{\mathbb{P}^{2}}^{\geq 2}$.

(c) $\mathbf{D}_{\mathbb{F}_{\delta}}^{2} \subseteq \mathbf{D}_{\mathbb{P}^{2}}$ for all $\delta>0$.

Proof. According to the proof of Theorem 2.5.4, for any $\delta>0$ it is satisfied that $\mathbf{d} \in \mathbf{D}_{\mathbb{P}^{2}}$ (respectively, $\mathbf{d} \in \mathbf{D}_{\mathbb{F}_{\delta}}^{1}$ ) if and only if $\beta_{1}^{\prime 2} \geq \bar{\beta}_{g+1} / \bar{\beta}_{0}^{2}$ (respectively, $2 \beta_{1}^{\prime}+$ $\left.\delta \beta_{1}^{\prime 2} \geq \bar{\beta}_{g+1} / \bar{\beta}_{0}^{2}\right)$. As we are supposing $\beta_{1}^{\prime 2} \geq \bar{\beta}_{g+1} / \bar{\beta}_{0}^{2}$, the inequality $2 \beta_{1}^{\prime}+\delta \beta_{1}^{\prime 2} \geq \beta_{1}^{\prime 2}$ is true when $\delta$ is a positive integer and also if $\delta=0$ and $\beta_{1}^{\prime}(\nu) \leq 2$, which proves (a) and the first inclusion in (b).

The second inclusion in (b) follows from an analogous argument which yields the opposite inequality.

To finish, using again Theorem 2.5.4, a class $\mathbf{d}$ is an element of $\mathbf{D}_{\mathbb{F}_{\delta}}^{2}, \delta>0$, if and only if $2 \beta_{1}^{\prime}-\delta \geq \bar{\beta}_{g+1} / \bar{\beta}_{0}^{2}$. Consequently, the fact that $\beta_{1}^{\prime 2} \geq 2 \beta_{1}^{\prime}-\delta$ concludes the proof.

Remark 2.5.6. The inclusions introduced in Theorem 2.5.5 are strict. Let us show this fact with some examples.

(a) Fix the discrete class $\mathbf{d}=(2,1,4 / 3,17 / 3,1)$. Therefore $e_{0}=9, e_{1}=3$ and $e_{2}=1$. Moreover,

$$
\beta_{1}^{\prime}=\frac{48}{27} \text { and } \sum_{i=0}^{g} \tau_{i}^{2}\left(\beta_{i+1}^{\prime}(\nu)-1\right)+\beta_{0}^{\prime}=\frac{50}{27}
$$

which shows that $\mathbf{d} \notin \mathbf{D}_{\mathbb{P}^{2}}$. Nevertheless,

$$
\beta_{1}^{\prime}\left(\delta \beta_{1}^{\prime}+2\right)=\frac{48 \delta+72}{27} .
$$

This proves that $\mathbf{d} \in \mathbf{D}_{\mathbb{F}_{\delta}}^{1}$ for all non-negative integer $\delta$. Consequently the inclusion in Theorem 2.5.5 (a) and the first one of Theorem 2.5.5 (b) are strict.

(b) Consider $\delta=0$ and the class $\mathbf{d}=(3,1,7 / 3,43 / 2,14 / 3,1)$. Thus $e_{0}=18, e_{1}=$ $6, e_{2}=3$ and $e_{3}=1$ and one obtains

$$
2 \beta_{1}^{\prime}=\frac{504}{108}, \sum_{j=0}^{g} \tau_{j}^{2}\left(\beta_{j+1}^{\prime}-1\right)+\beta_{0}^{\prime}=\frac{509}{108} \text { and } \beta_{1}^{\prime 2}=\frac{588}{108}
$$

which implies that $\mathbf{d} \in \mathbf{D}_{\mathbb{P}^{2}}^{\geq 2}$ but $\mathbf{d} \notin \mathbf{D}_{\mathbb{F}_{0}}^{1, \geq 2}$. 
(c) To conclude, set the class $\mathbf{d}=(2,1,5 / 2,57 / 5, \Phi)$, where $\Phi$ denotes the golden ratio. One has $e_{0}=10, e_{1}=5$ and $e_{2}=1$. Then

$$
2 \beta_{1}^{\prime}-\delta=\frac{100}{20}-\delta \text { and } \sum_{j=0}^{g} \tau_{j}^{2}\left(\beta_{j+1}^{\prime}-1\right)+\beta_{0}^{\prime}=\frac{102}{20}+\frac{1}{100}(\Phi-1) .
$$

This shows that $\mathbf{d} \notin \mathbf{D}_{\mathbb{F}_{\delta}}^{2}$ for any positive integer $\delta$, although $\mathbf{d} \in \mathbf{D}_{\mathbb{P}^{2}}$ since $\beta_{1}^{\prime 2}$ $=125 / 20$.

\subsubsection{An algorithm for obtaining the dual graphs of NPI valuations}

The aim of this subsection is to describe a procedure in order to generate those discrete classes (that is, dual graphs) admitting some NPI divisorial or irrational valuation. We assume $g>0$ because any discrete class $\left(0,1, \beta_{1}^{\prime}\right)$ admits an NPI (respectively, NPI special) valuation of $\mathbb{P}^{2}$ (respectively, $\mathbb{F}_{\delta}$ ) and by Theorem 2.5.4 the discrete classes $\left(0,1, \beta_{1}^{\prime} \geq \delta\right)$ are those that admit NPI non-special valuations of $\mathbb{F}_{\delta}$.

Our algorithm starts with an input

$$
\mathbf{d}\left(\nu_{I}\right)=\left(g, \beta_{0}^{\prime}\left(\nu_{I}\right), \beta_{1}^{\prime}\left(\nu_{I}\right), \ldots, \beta_{g+1}^{\prime}\left(\nu_{I}\right)=1\right),
$$

which is a discrete class belonging to $\mathbf{D}_{\mathbb{P}^{2}}, \mathbf{D}_{\mathbb{F}_{\delta}}^{1}$ or $\mathbf{D}_{\mathbb{F}_{\delta}}^{2}$, simply written by $\mathbf{D}$. It provides two outputs:

Output 1. Another discrete class of the same set $\mathbf{D}$ of the input of the form:

$$
\begin{aligned}
\mathbf{d}\left(\nu_{O_{1}}\right)=\left(g+1, \beta_{0}^{\prime}\left(\nu_{O_{1}}\right)\right. & =\beta_{0}^{\prime}\left(\nu_{I}\right), \beta_{1}^{\prime}\left(\nu_{O_{1}}\right)=\beta_{1}^{\prime}\left(\nu_{I}\right), \\
& \left.\ldots, \beta_{g}^{\prime}\left(\nu_{O_{1}}\right)=\beta_{g}^{\prime}\left(\nu_{I}\right), \beta_{g+1}^{\prime}\left(\nu_{O_{1}}\right), \beta_{g+2}^{\prime}\left(\nu_{O_{1}}\right)=1\right) .
\end{aligned}
$$

Output 2. In fact, it is a double output. The first one is a discrete class in the same set $\mathbf{D}$ of the input as follows:

$$
\begin{aligned}
\mathbf{d}\left(\nu_{O_{2}^{1}}\right)=\left(g, \beta_{0}^{\prime}\left(\nu_{O_{2}^{1}}\right)=\beta_{0}^{\prime}\left(\nu_{O_{1}}\right), \beta_{1}^{\prime}\left(\nu_{O_{2}^{1}}\right)\right. & =\beta_{1}^{\prime}\left(\nu_{O_{1}}\right), \\
\ldots, & \left.\beta_{g}^{\prime}\left(\nu_{O_{2}^{1}}\right)=\beta_{g}^{\prime}\left(\nu_{O_{1}}\right), \beta_{g+1}^{\prime}\left(\nu_{O_{2}^{1}}\right)\right),
\end{aligned}
$$

where $\beta_{g+1}^{\prime}\left(\nu_{O_{2}^{1}}\right) \in \mathbb{R}_{>0} \backslash \mathbb{Q}$.

And the second one is a discrete class in the same set $\mathbf{D}$ of the input with the following shape

$$
\begin{aligned}
\mathbf{d}\left(\nu_{O_{2}^{2}}\right)=\left(g+1, \beta_{0}^{\prime}\left(\nu_{O_{2}^{2}}\right)=\beta_{0}^{\prime}\left(\nu_{O_{1}}\right), \beta_{1}^{\prime}\left(\nu_{O_{2}^{2}}\right)=\beta_{1}^{\prime}\left(\nu_{O_{1}}\right),\right. \\
\left.\ldots, \beta_{g+1}^{\prime}\left(\nu_{O_{2}^{2}}\right)=\beta_{g+1}^{\prime}\left(\nu_{O_{1}}\right), \beta_{g+2}^{\prime}\left(\nu_{O_{1}^{2}}\right)\right),
\end{aligned}
$$

$\beta_{g+2}^{\prime}\left(\nu_{O_{1}^{2}}\right)$ being a positive integer different from 1.

Note that the outputs are not unique. In fact, one can obtain infinitely tuples as Output 1 and also as the first one in Output 2. 
It is easily seen that the tuple of the input corresponds to a discrete class of an NPI divisorial valuation which is defined by a satellite (exceptional) divisor whose dual graph $\Gamma$ has subgraphs $\Gamma^{j}, 1 \leq j \leq g$, and $\Gamma^{g+1}$ that contains only the vertex $\operatorname{st}_{g}$. The algorithm gives us dual graphs admitting the same type of NPI valuations which maintain the subgraphs $\Gamma^{j}, 1 \leq j \leq g$, and have a new subgraph $\Gamma^{g+1}$; the obtained graphs correspond to divisorial valuations defined by a satellite divisor or to irrational valuations. In addition, our algorithm also provides dual graphs of NPI divisorial valuations defined by free divisors which preserve the subgraphs $\Gamma^{j}, 1 \leq j \leq g$, and add two subgraphs more, $\Gamma^{g+1}$ and (a tail) $\Gamma^{g+2}$.

It becomes clear that selecting appropriate tuples $\left(1, \beta_{0}^{\prime}=1, \beta_{1}^{\prime}, 1\right)$ at the beginning, we are able to give the dual graph of any NPI valuation of any desired type with $g$ as large as we want.

Let us show our algorithm. For a start and under the notation stated before, set

$$
q(\mathbf{d}(\nu)):=\left\{\begin{array}{cl}
\beta_{1}^{\prime}(\nu)^{2}, & \text { if } Z_{0}=\mathbb{P}^{2}, \\
\beta_{1}^{\prime}(\nu)\left(\delta \beta_{1}^{\prime}(\nu)+2\right), & \text { if } Z_{0}=\mathbb{F}_{\delta} \text { and } \nu \text { is special, } \\
2 \beta_{1}^{\prime}(\nu)-\delta, & \text { if } Z_{0}=\mathbb{F}_{\delta} \text { and } \nu \text { is non-special, }
\end{array}\right.
$$

$\beta_{1}^{\prime}(\nu)$ being the third coordinate of $\mathbf{d}(\nu)$.

Input: A discrete class $\mathbf{d}\left(\nu_{I}\right)$ as in (2.18) that satisfies

$$
q\left(\mathbf{d}\left(\nu_{I}\right)\right)>\sum_{j=0}^{g-1} e_{j}\left(\nu_{I}^{N}\right)^{2}\left(\beta_{j+1}^{\prime}\left(\nu_{I}\right)-1\right)+\beta_{0}^{\prime}\left(\nu_{I}\right) .
$$

This condition must be imposed since we start with the discrete class of an NPI divisorial valuation and one needs to have some degree of freedom to add Puiseux exponents.

Output 1: It will be a tuple $\mathbf{d}\left(\nu_{O_{1}}\right)$ as in (2.19) computed as follows. Set $\beta_{g+1}^{\prime}\left(\nu_{O_{1}}\right)=$ $q_{g+1} / n_{g+1}$, where $q_{g+1}, n_{g+1} \in \mathbb{Z}_{>0}$ are such that $\operatorname{gcd}\left(q_{g+1}, n_{g+1}\right)=1$ and $q_{g+1}>$ $n_{g+1}$. The output must satisfy

$$
\beta_{j}^{\prime}\left(\nu_{O_{1}}\right)=\beta_{j}^{\prime}\left(\nu_{I}\right) \text { and } e_{j}\left(\nu_{O_{1}}^{N}\right)=e_{j}\left(\nu_{I}^{N}\right), \text { for } 0 \leq j \leq g,
$$

and $\bar{\beta}_{0}\left(\nu_{O_{1}}\right)=n_{g+1} \bar{\beta}_{0}\left(\nu_{I}\right)$. Then

$$
e_{g+1}\left(\nu_{O_{1}}^{N}\right)=\frac{1}{\bar{\beta}_{0}\left(\nu_{O_{1}}\right)}=\frac{1}{n_{g+1} \bar{\beta}_{0}\left(\nu_{I}\right)}=\frac{e_{g}\left(\nu_{I}^{N}\right)}{n_{g+1}} .
$$

As we desire that the tuple $\mathbf{d}\left(\nu_{O_{1}}\right)$ corresponds to a discrete class of an NPI valuation, for obtaining a suitable Output 1 it suffices to take a pair $q_{g+1}$ and $n_{g+1}$ (which defines $\left.\beta_{g+1}^{\prime}\left(\nu_{O_{1}}\right)\right)$ satisfying the following inequality:

$$
\begin{aligned}
& \frac{q\left(\mathbf{d}\left(\nu_{I}\right)\right)-\sum_{j=0}^{g-1} e_{j}\left(\nu_{I}^{N}\right)^{2}\left(\beta_{j+1}^{\prime}\left(\nu_{I}\right)-1\right)-\beta_{0}^{\prime}\left(\nu_{I}\right)}{e_{g}\left(\nu_{I}^{N}\right)^{2}}+1 \geq \beta_{g+1}^{\prime}\left(\nu_{O_{1}}\right)= \\
& \frac{q_{g+1}}{n_{g+1}}>1 .
\end{aligned}
$$


Note that the algorithm must make a choice since there are infinitely many options.

Output 2: To obtain a tuple $\mathbf{d}\left(\nu_{\mathrm{O}_{2}^{1}}\right)$ as (2.20), it suffices to search an irrational number $\beta_{g+1}^{\prime}\left(\nu_{O_{2}^{1}}\right)$ which satisfies Inequality $(2.23)$ when we replace the rational number $\beta_{g+1}^{\prime}\left(\nu_{O_{1}}\right)$ with $\beta_{g+1}^{\prime}\left(\nu_{O_{2}^{1}}\right)$. Notice that we have to make a choice again.

Finally, for getting a discrete class $\mathbf{d}\left(\nu_{O_{2}^{2}}\right)$ as (2.21), it suffices to find a positive integer $\beta_{g+2}^{\prime}\left(\nu_{O_{2}^{2}}\right)>1$ such that

$$
\frac{q\left(\mathbf{d}\left(\nu_{O_{2}^{2}}\right)\right)-\sum_{j=0}^{g} e_{j}\left(\nu_{O_{1}}^{N}\right)^{2}\left(\beta_{j+1}^{\prime}\left(\nu_{O_{1}}\right)-1\right)-\beta_{0}^{\prime}\left(\nu_{O_{1}}\right)}{e_{g+1}\left(\nu_{O_{1}}^{N}\right)^{2}} \geq \beta_{g+2}^{\prime}\left(\nu_{O_{2}^{2}}\right)-1 .
$$

We remark that the biggest non-negative integer $\beta_{g+2}^{\prime}\left(\nu_{O_{2}^{2}}\right)-1$ satisfying Inequality (2.24) provides the maximum number of free vertices one can obtain in the tail of the dual graph.

To conclude this subsection, we give two examples which show how our algorithm runs.

Example 2.5.7. Consider $Z_{0}=\mathbb{P}^{2}$ and the input $\mathbf{d}\left(\nu_{I}\right)=(2,1,5 / 2,7 / 5,1)$. Therefore $\left\{e_{i}\left(\nu_{I}\right)\right\}_{i=0}^{2}=\{10,5,1\}$. As required, our input satisfied Inequality (2.22) since

$$
\frac{25}{4}=\left(\frac{5}{2}\right)^{2}>\left(\frac{5}{2}-1\right)+\left(\frac{1}{2}\right)^{2}\left(\frac{7}{5}-1\right)+1=\frac{52}{20} .
$$

Now suitable values for our purposes $\beta_{3}^{\prime}\left(\nu_{O_{1}}\right)$ are those satisfying Inequality (2.23), that is those $\beta_{3}^{\prime}\left(\nu_{O_{1}}\right)$ such that

$$
\begin{aligned}
& 366=\frac{\beta_{1}^{\prime}\left(\nu_{I}\right)^{2}-e_{0}\left(\nu_{I}^{N}\right)^{2}\left(\beta_{1}^{\prime}\left(\nu_{I}\right)-1\right)-e_{1}\left(\nu_{I}^{N}\right)^{2}\left(\beta_{2}^{\prime}\left(\nu_{I}\right)-1\right)-\beta_{0}^{\prime}\left(\nu_{I}\right)}{e_{2}\left(\nu_{I}^{N}\right)^{2}}+1 \geq \\
& \beta_{3}^{\prime}\left(\nu_{O_{1}}\right)>1 .
\end{aligned}
$$

If, for example, we select $\mathbf{d}\left(\nu_{O_{1}}\right)=(3,1,5 / 2,7 / 5,8 / 3,1)$, we get a valid Output 1. Our algorithm also searches values $\beta_{4}^{\prime}\left(\nu_{O_{2}^{2}}\right)$, which must satisfy Inequality (2.24), that is $3270 \geq \beta_{4}^{\prime}\left(\nu_{O_{2}^{2}}\right)-1 \geq 0$. Consequently a possible Output 2 would be $\mathbf{d}\left(\nu_{O_{2}^{2}}\right)=$ $(3,1,5 / 2,7 / 5,8 / 3,3200)$. The dual graphs of $\nu_{I}, \nu_{O_{1}}$ and $\nu_{O_{2}^{2}}$ can be seen in Figure 2.3 .

Example 2.5.8. Assume now that $Z_{0}=\mathbb{F}_{2}$ and $\mathbf{d}\left(\nu_{I}\right)=(3,1,5 / 3,12 / 5,5 / 2,1)$. This input is as the algorithm requires because $\left\{e_{i}\left(\nu_{I}\right)\right\}_{i=0}^{2}=\{30,10,2,1\}$,

$$
\frac{80}{9}=\frac{5}{3}\left(2 \frac{5}{3}+2\right)>\left(\frac{5}{3}-1\right)+\left(\frac{1}{3}\right)^{2}\left(\frac{12}{5}-1\right)+\left(\frac{1}{15}\right)^{2}\left(\frac{5}{2}-1\right)+1=\frac{823}{450}
$$

and, then, Inequality (2.22) holds. Thus, if we desire an output $\mathbf{d}\left(\nu_{O_{2}^{1}}\right)$, we need to find some irrational number such that it satisfies Inequality (2.23). That is, we can use any value $\beta_{4}^{\prime}\left(\nu_{O_{2}^{1}}\right)$ such that $6355 \geq \beta_{4}^{\prime}\left(\nu_{O_{2}^{1}}\right)>0$. Therefore, $\mathbf{d}\left(\nu_{O_{2}^{1}}\right)=$ $(3,1,5 / 3,12 / 5,5 / 2, \pi)$ is a suitable output. One can see the dual graphs of $\nu_{I}$ and $\nu_{O_{2}^{1}}$ in Figure 2.4. 


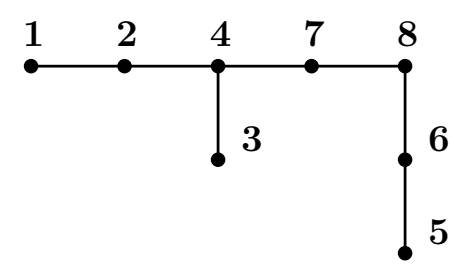

(a) Dual graph of $\nu_{I}$

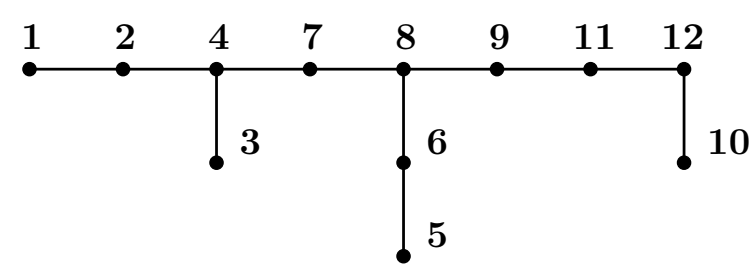

(b) Dual graph of $\nu_{O_{1}}$

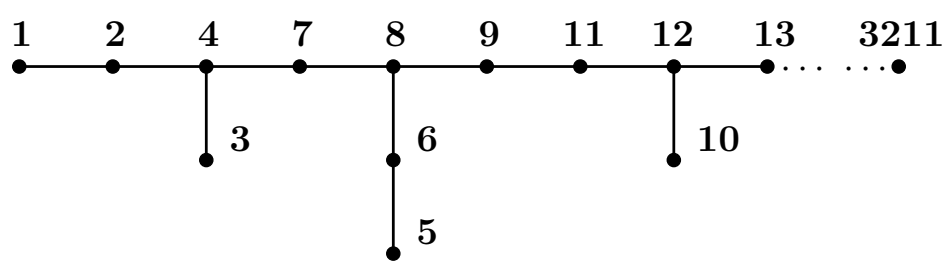

(c) Dual graph of $\nu_{O_{2}^{2}}$

Figure 2.3: Dual graphs in Example 2.5.7.

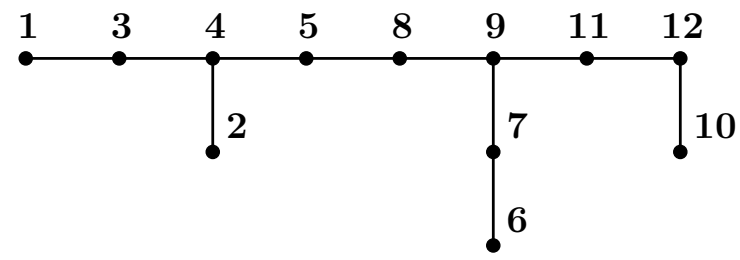

(a) Dual graph of $\nu_{I}$

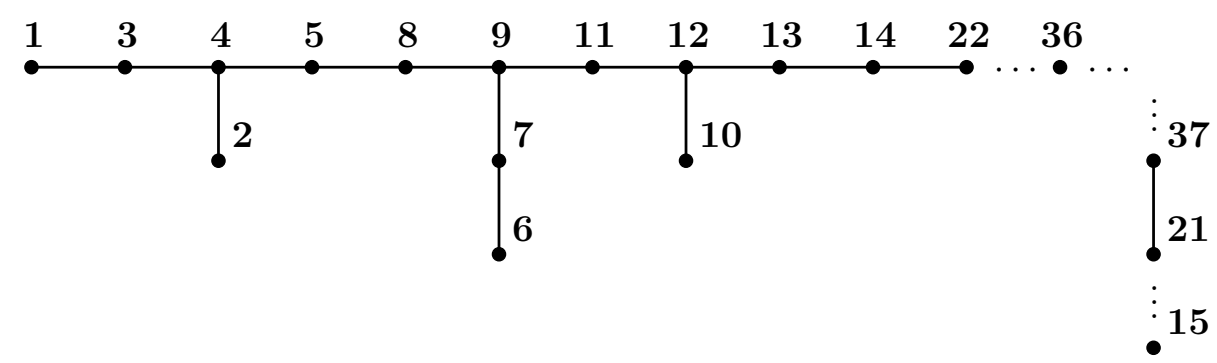

(b) Dual graph of $\nu_{O_{2}^{1}}$

Figure 2.4: Dual graphs in Example 2.5.8. 


\section{Chapter 3}

\section{Seshadri-type constants and Newton-Okounkov bodies for non-positive at infinity valuations}

The aim of this chapter is to describe the Seshadri-type constants and NewtonOkounkov bodies with respect to non-positive at infinity valuations of Hirzebruch surfaces. Our main results and proofs concerning these objects can be found in [62]. This chapter also contains the study of the same constants and bodies when the valuations are of the projective plane. As a reference, we have used the articles [64] and [65]. Recall that a basic introduction about Seshadri-type constants and Newton-Okounkov bodies for surfaces was provided in Section 1.5. We keep the notation of the previous chapters. In this chapter $k$ will be the complex field $\mathbb{C}$.

Denote by $Z_{0}$ the projective plane $\mathbb{P}^{2}$ or a Hirzebruch surface $\mathbb{F}_{\delta}, \delta \geq 0$, over $\mathbb{C}$ and $p$ a point of $Z_{0}$. Let $\nu$ be an exceptional curve valuation of the function field of $Z_{0}$ centered at $\mathcal{O}_{Z_{0}, p}$. Write $\nu_{n}$ the divisorial valuation corresponding to a finite simple sequence of blowups as follows:

$$
\pi: Z:=Z_{n} \stackrel{\pi_{n}}{\longrightarrow} Z_{n-1} \rightarrow \ldots \rightarrow Z_{1} \stackrel{\pi_{1}}{\longrightarrow} Z_{0}
$$

The valuation $\nu$ has a distinguished point $p_{r}$ such that $p_{i} \rightarrow p_{r}$ for $i>r$ and its first component, which is a divisorial valuation (Theorem 1.3.2), is defined by the divisor $E_{r}$, thus it is usually denoted $\nu_{r}$ (see Section 1.3). As in Chapter 2, for simplicity, we denote by $E_{i}$ (respectively, $E_{i}^{*}$ ) the strict (respectively, total) transform on $Z_{n}$ of the exceptional divisor $E_{i}$ created after blowing-up $p_{i}$ and by $\tilde{D}$ (respectively, $D^{*}$ ) the strict (respectively, total) transform on $Z_{n}$ of a divisor $D$ on $Z_{i}$, for $i \leq n$. Write $\varphi_{C}$ (respectively, $\varphi_{i}$ ) the germ of a curve $C$ at $p$ (respectively, an analytically irreducible germ at $p$ whose strict transform on $Z_{i}$ is transversal to $E_{i}$ at a non-singular point of the exceptional locus).

Keeping the conventions of the above chapters, $\nu$ (respectively, $\nu_{n}$ ) will be often 
called exceptional curve (respectively, divisorial) valuations of $Z_{0}$. If $Z_{0}=\mathbb{P}^{2}$, and one considers a divisorial valuation $\nu_{n}, L$ denotes the line at infinity (see Section 2.1). In the case $Z_{0}=\mathbb{F}_{\delta}, F_{1}$ denotes the fiber containing $p, M_{0}$ denotes the special section and, when $\nu_{n}$ is non-special, $M_{1}$ denotes the irreducible curve of degree $(0,1)$ going through $p$ whose strict transform has negative self-intersection on the surface defined by $\nu_{n}$ (see Proposition 2.2.2).

Set again $\nu_{n}$ a divisorial valuation of $Z_{0}$ and consider a big divisor $D$ on $Z_{0}$. Recall that the value $\hat{\mu}_{D}\left(\nu_{n}\right)$ introduced in Subsection 1.5.1 is

$$
\hat{\mu}_{D}\left(\nu_{n}\right)=\lim _{m \rightarrow \infty} \frac{\max \left\{\nu_{n}(f) \mid f \in H^{0}\left(Z_{0}, \mathcal{O}_{Z_{0}}(m D)\right)\right\}}{m} .
$$

In addition, this value $\hat{\mu}_{D}\left(\nu_{n}\right)$ has a lower bound (1.10) which can be written as

$$
\hat{\mu}_{D}\left(\nu_{n}\right) \geq \sqrt{\operatorname{vol}_{Z_{0}}(D) \bar{\beta}_{g+1}\left(\nu_{n}\right)},
$$

because $\bar{\beta}_{g+1}\left(\nu_{n}\right)=\operatorname{vol}\left(\nu_{n}\right)^{-1}$ by [64, Section 2.5].

We conclude this brief introduction with the concept of minimal valuation (of $\mathbb{P}^{2}$ o $\mathbb{F}_{\delta}$ ) with respect to a big divisor.

Definition 3.0.1. Let $D$ be a big divisor on a surface $Z_{0}$ as defined above. A divisorial valuation $\nu_{n}$ of $Z_{0}$ is called to be minimal with respect to $D$ if

$$
\hat{\mu}_{D}\left(\nu_{n}\right)=\sqrt{\operatorname{vol}_{Z_{0}}(D) \bar{\beta}_{g+1}\left(\nu_{n}\right)} .
$$

When the above condition is not satisfied, the valuation $\nu_{n}$ is named non-minimal with respect to $D$.

\subsection{Seshadri-type constants for divisorial valuations of the projective plane}

In this section we are going to give a definition of minimal divisorial valuation of $\mathbb{P}^{2}:=\mathbb{P}_{\mathbb{C}}^{2}$ which does not depend on a divisor. It can be found in [15, 38] and [64]. We will prove in Proposition 3.1.3 that this definition is equivalent to Definition 3.0.1 in the projective case and the reference to a divisor can be deleted. Moreover, we will show interesting results related to non-minimal valuations of $\mathbb{P}^{2}$.

Assume that $Z_{0}=\mathbb{P}^{2}:=\mathbb{P}_{\mathbb{C}}^{2}$. Consider projective coordinates $(X: Y: Z)$ in $\mathbb{P}^{2}$, a point $p \in \mathbb{P}^{2}$ with coordinates $(1: 0: 0)$ and the affine coordinates $u=Y / X$ and $v=Z / X$ around $p$. Let $\nu_{n}$ be a divisorial valuation of the function field of $\mathbb{P}^{2}$ centered at $\mathcal{O}_{\mathbb{P}^{2}, p}$ and set $E_{0}$ a general projective line on $\mathbb{P}^{2}$. Following [38] and [64], the value $\hat{\mu}\left(\nu_{n}\right)$ is defined as

$$
\hat{\mu}\left(\nu_{n}\right):=\lim _{m \rightarrow \infty} \frac{\mu_{m}\left(\nu_{n}\right)}{m},
$$


where

$$
\begin{aligned}
\mu_{m}\left(\nu_{n}\right) & =\max \left\{\nu_{n}(f) \mid f \in H^{0}\left(Z_{0}, \mathcal{O}_{Z_{0}}\left(m E_{0}\right)\right)\right\} \\
& =\max \left\{\nu_{n}(f) \mid f \in \mathbb{C}[u, v], \operatorname{deg}(f) \leq m\right\} .
\end{aligned}
$$

Taking into account (3.1) and (3.2), it holds that $\hat{\mu}\left(\nu_{n}\right) \geq \sqrt{\bar{\beta}_{g+1}\left(\nu_{n}\right)}$.

Now we introduce the above mentioned concept of minimal valuation of $\mathbb{P}^{2}$.

Definition 3.1.1. [38, 64] A divisorial valuation $\nu_{n}$ of $\mathbb{P}^{2}$ is called to be minimal if $\hat{\mu}\left(\nu_{n}\right)=\sqrt{\bar{\beta}_{g+1}\left(\nu_{n}\right)}$.

Notice that a divisorial valuation of $\mathbb{P}^{2}$ is minimal in the sense of the above definition if and only if it is minimal with respect to $E_{0}$ in the sense of Definition 3.0.1.

The following lemma will allow us to show the relation between our definitions 3.0.1 and 3.1.1 in the projective case.

Lemma 3.1.2. Let $\nu_{n}$ be a divisorial valuation of $\mathbb{P}^{2}$ and $d$ a positive integer. Then

$$
\hat{\mu}_{d E_{0}}\left(\nu_{n}\right)=d \hat{\mu}\left(\nu_{n}\right)
$$

Proof. Taking into account (3.1), it holds that

$$
\begin{aligned}
\hat{\mu}_{d E_{0}}\left(\nu_{n}\right) & =\lim _{m \rightarrow \infty} \frac{\max \left\{\nu_{n}(f) \mid f \in \mathbb{C}[u, v], \operatorname{deg}(f) \leq d m\right\}}{m} \\
& =d \lim _{m^{\prime} \rightarrow \infty} \frac{\max \left\{\nu_{n}(f) \mid f \in \mathbb{C}[u, v], \operatorname{deg}(f) \leq m^{\prime}\right\}}{m^{\prime}} \\
& =d \hat{\mu}\left(\nu_{n}\right),
\end{aligned}
$$

where the second equality follows from the replacement $m^{\prime}=d m$ and the third one from (3.3).

Proposition 3.1.3. Let $\nu$ be a divisorial valuation of $\mathbb{P}^{2}$. Then $\nu_{n}$ is minimal in the sense of Definition 3.1 .1 if and only if it is minimal with respect to any big divisor $D$ on $\mathbb{P}^{2}$ in the sense of Definition 3.0.1.

Proof. By Definition 3.1.1, a minimal divisorial valuation of $\mathbb{P}^{2}$ satisfies

$$
\hat{\mu}\left(\nu_{n}\right)^{2}=\bar{\beta}_{g+1}\left(\nu_{n}\right) .
$$

Consequently, it holds that $d^{2} \hat{\mu}\left(\nu_{n}\right)^{2}=d^{2} \bar{\beta}_{g+1}\left(\nu_{n}\right)$, for all positive integers $d$. In addition, by Lemma 3.1.2, the equality $\hat{\mu}_{D}\left(\nu_{n}\right)^{2}=D^{2} \bar{\beta}_{g+1}\left(\nu_{n}\right)$ holds, where $D$ is a big divisor linearly equivalent to $d E_{0}$, which completes the proof.

We finish this section giving two results concerning non-minimal divisorial valuations of $\mathbb{P}^{2}$. 
Proposition 3.1.4. [[38, Lemma 5.1] and [65, Lemma 3.10]] Let $\nu_{n}$ be a divisorial valuation of $\mathbb{P}^{2}$. Suppose the existence of an irreducible polynomial $f \in \mathbb{C}[u, v]$ such that $\nu_{n}(f)>\operatorname{deg}(f) \sqrt{\bar{\beta}_{g+1}\left(\nu_{n}\right)}$. Then

$$
\hat{\mu}\left(\nu_{n}\right)=\frac{\nu_{n}(f)}{\operatorname{deg}(f)} .
$$

In addition, if $\nu_{n}$ is a non-minimal valuation, then there exists such an irreducible polynomial $f$ and it is the unique irreducible polynomial (up to product by a non-zero constant) satisfying the above condition.

Definition 3.1.5. Let $\nu_{n}$ be a non-minimal divisorial valuation of $\mathbb{P}^{2}$. A curve $C$ is called supraminimal of $\nu_{n}$ if it is defined by an irreducible polynomial $f \in \mathbb{C}[u, v]$ satisfying $\nu_{n}(f) / \operatorname{deg}(f)=\hat{\mu}\left(\nu_{n}\right)$. This curve is unique by the above proposition.

Corollary 3.1.6. Let $\nu_{n}$ be a non-minimal divisorial valuation of $\mathbb{P}^{2}$ and $Z$ the surface which $\nu_{n}$ defines. Then the class of the strict transform of the supraminimal curve of $\nu_{n}$ generates an extremal ray of the cone of curves $\mathrm{NE}(Z)$ of $Z$.

Proof. To prove the result we show that the strict transform of the supraminimal curve of $\nu_{n}$ has negative self-intersection and, by Proposition 1.4.6, we conclude the proof.

Consider the divisor

$$
D=E_{0}^{*}-\frac{1}{\hat{\mu}\left(\nu_{n}\right)} \sum_{i=1}^{n} \nu_{n}\left(\mathfrak{m}_{i}\right) E_{i}^{*}
$$

This divisor is nef and big. Indeed, suppose that $\tilde{C}_{h}$ is the strict transform on $Z$ of a curve $C_{h}$ on $\mathbb{P}^{2}$ defined by a polynomial $h \in \mathbb{C}[u, v]$. Then

$$
D \cdot \tilde{C}_{h}=\operatorname{deg}(h)-\frac{1}{\hat{\mu}\left(\nu_{n}\right)} \sum_{i=1}^{n} \nu_{n}\left(\mathfrak{m}_{i}\right) \cdot \operatorname{mult}_{p_{i}}(h)=\operatorname{deg}(h)-\frac{\nu_{n}(h)}{\hat{\mu}\left(\nu_{n}\right)} \geq 0,
$$

where the second equality holds by Noether's formula and the inequality by the fact that $\nu_{n}$ is non-minimal. In addition, by the proximities equalities, $D \cdot E_{i}=0$, for all $i \in\{1,2, \ldots, n\}$. Therefore, we have just proved that $D$ is nef. The self-intersection of the divisor $D$ satisfies

$$
D^{2}=\left(E_{0}^{*}\right)^{2}-\frac{\bar{\beta}_{g+1}\left(\nu_{n}\right)}{\hat{\mu}\left(\nu_{n}\right)^{2}}>1-1=0
$$

by assumption, and by Theorem $1.4 .7, D$ is big.

Now, denote by $\tilde{C}_{f}$ the strict transform of the supraminimal curve of $\nu_{n} C_{f} . C_{f}$ is defined by an irreducible polynomial $f \in \mathbb{C}[u, v]$. Consequently, by Proposition 3.1.4,

$$
D \cdot \tilde{C}=\operatorname{deg}(f)-\frac{\nu_{n}\left(\varphi_{C}\right)}{\hat{\mu}\left(\nu_{n}\right)}=\operatorname{deg}(f)-\operatorname{deg}(f)=0,
$$

and so $\tilde{C}_{f}$ is orthogonal to $D$. This implies that $\tilde{C}_{f}$ has negative self-intersection by Proposition 1.4.12, which completes the proof. 
Remark 3.1.7. Let $\nu_{n}$ be a non-positive at infinity divisorial valuation of $\mathbb{P}^{2}$. By [64, Proposition 5.4], $\hat{\mu}\left(\nu_{n}\right)=\nu_{n}\left(\varphi_{L}\right)$ and the line at infinity $L$ is the supraminimal curve of $\nu_{n}$ when $\nu_{n}$ is non-minimal.

\subsection{Seshadri-type constants for NPI divisorial valuations of Hirzebruch surfaces}

We devote this subsection to study the value $\hat{\mu}_{D}\left(\nu_{n}\right)$ for any non-positive at infinity divisorial valuation $\nu_{n}$ of a Hirzebruch surface $\mathbb{F}_{\delta}$ over $\mathbb{C}$ and any big divisor $D$ on $\mathbb{F}_{\delta}$. We will also show some consequences of our study.

Our main result is the following one:

Theorem 3.2.1. Let $\nu_{n}$ be an NPI divisorial valuation of the function field of $\mathbb{F}_{\delta}$ centered at $\mathcal{O}_{\mathbb{F}_{\delta}, p}$. Set $D \sim a F+b M$ a big divisor on $\mathbb{F}_{\delta}$. Then:

(a) If $\nu_{n}$ is special, then $\hat{\mu}_{D}\left(\nu_{n}\right)=(a+b \delta) \nu_{n}\left(\varphi_{F_{1}}\right)+b \nu_{n}\left(\varphi_{M_{0}}\right)$.

(b) Otherwise, $\hat{\mu}_{D}\left(\nu_{n}\right)=a \nu_{n}\left(\varphi_{F_{1}}\right)+b \nu_{n}\left(\varphi_{M_{1}}\right)$.

Proof. To show (a) we assume that $p$ is a special point. When $p$ is a point of $\mathbb{F}_{0}$ (respectively, $p$ is a general point), the proof is similar and holds assuming $\delta=0$ (respectively, $\nu_{n}\left(\varphi_{M_{0}}\right)=0$ ). Set $C$ a curve on $\mathbb{F}_{\delta}$ such that $C \in|m D|$, where $m \in \mathbb{Z}_{>0}$, and $\tilde{C}$ its strict transform on $Z$. The divisor on $Z$

$$
\Lambda\left(\nu_{n}\right)=\nu_{n}\left(\varphi_{M_{0}}\right) F^{*}+\nu_{n}\left(\varphi_{F_{1}}\right) M^{*}-\sum_{i=1}^{n} \nu_{n}\left(\mathfrak{m}_{i}\right) E_{i}^{*}
$$

is nef by Theorem 2.3.7 and so $\Lambda\left(\nu_{n}\right) \cdot \tilde{C} \geq 0$. This implies that

$$
(a+b \delta) \nu_{n}\left(\varphi_{F_{1}}\right)+b \nu_{n}\left(\varphi_{M_{0}}\right) \geq \frac{\nu_{n}\left(\varphi_{C}\right)}{m}
$$

and consequently we have obtained an upper bound for $\nu_{n}\left(\varphi_{C}\right) / m$, where $C$ belongs to $|m D|$ and $m \in \mathbb{Z}_{>0}$. Now fix the curve $C_{1}=m(a+\delta b) F_{1}+m b M_{0}$ and then

$$
C_{1} \in|m D| \text { and } \frac{\nu_{n}\left(\varphi_{C_{1}}\right)}{m}=(a+\delta b) \nu_{n}\left(\varphi_{F_{1}}\right)+b \nu_{n}\left(\varphi_{M_{0}}\right)
$$

which proves (a) since we have just seen that the bound can be reached.

Likewise a proof for (b) follows from considering the divisor

$$
\Delta\left(\nu_{n}\right)=\left(\nu_{n}\left(\varphi_{M_{1}}\right)-\delta \nu_{n}\left(\varphi_{F_{1}}\right)\right) F^{*}+\nu_{n}\left(\varphi_{F_{1}}\right) M^{*}-\sum_{i=1}^{n} \nu_{n}\left(\mathfrak{m}_{i}\right) E_{i}^{*},
$$

which is nef by Theorem 2.4.8, and the curve $C_{1}=m a F_{1}+m b M_{1}$. 
Remark 3.2.2. Let $\nu_{n}$ be an NPI divisorial valuation of $\mathbb{P}^{2}$ and $\nu$ the corresponding NPI special divisorial valuation of $\mathbb{F}_{1}$ described in the proof of Proposition 2.3.3. Set $E_{0}$ a general projective line on $\mathbb{P}^{2}$ and $M$ an irreducible curve of degree $(0,1)$ on $\mathbb{F}_{1}$. The proofs of Proposition 2.3.3 and Theorem 3.2.1 prove that

$$
\hat{\mu}\left(\nu_{n}\right)=\hat{\mu}_{E_{0}}\left(\nu_{n}\right)=\nu_{n}\left(\varphi_{L}\right)=\nu\left(\varphi_{F_{1}}\right)+\nu\left(\varphi_{M_{0}}\right)=\hat{\mu}_{M}(\nu) .
$$

Even more, by Remark 2.3.5, if $\nu_{n}$ is minimal then $\nu$ is non-minimal with respect to $M$ since

$$
\hat{\mu}_{M}(\nu)=\hat{\mu}\left(\nu_{n}\right)=\sqrt{\bar{\beta}_{g+1}\left(\nu_{n}\right)}=\sqrt{\bar{\beta}_{\hat{g}+1}(\nu)+\nu\left(\varphi_{M_{0}}\right)^{2}}>\sqrt{\bar{\beta}_{\hat{g}+1}(\nu)},
$$

where $\bar{\beta}_{g+1}\left(\nu_{n}\right)$ (respectively, $\bar{\beta}_{\hat{g}+1}(\nu)$ ) is the last maximal contact value of $\nu_{n}$ (respectively, $\nu)$.

Corollary 3.2.3. Let $\nu_{n}$ be an NPI divisorial valuation of $\mathbb{F}_{\delta}$ and set $D \sim a F+b M$ a big and nef divisor on $\mathbb{F}_{\delta}$. Then

(a) When $\nu_{n}$ is special, it is minimal with respect to $D$ if and only if

$$
2 \nu_{n}\left(\varphi_{M_{0}}\right) \nu_{n}\left(\varphi_{F_{1}}\right)+\delta \nu_{n}\left(\varphi_{F_{1}}\right)^{2}=\operatorname{vol}\left(\nu_{n}\right)^{-1}
$$

and $a=b \nu_{n}\left(\varphi_{M_{0}}\right) / \nu_{n}\left(\varphi_{F_{1}}\right)$.

(b) Otherwise, $\nu_{n}$ is minimal with respect to $D$ if and only if

$$
2 \nu_{n}\left(\varphi_{M_{1}}\right) \nu_{n}\left(\varphi_{F_{1}}\right)-\delta \nu_{n}\left(\varphi_{F_{1}}\right)^{2}=\operatorname{vol}\left(\nu_{n}\right)^{-1}
$$

and $a=b\left(\nu_{n}\left(\varphi_{M_{1}}\right)-\delta \nu_{n}\left(\varphi_{F_{1}}\right)\right) / \nu_{n}\left(\varphi_{F_{1}}\right)$.

Proof. We will see (a). Applying a similar argument, one can prove (b).

We begin by proving that $\nu_{n}$ is minimal with respect to $D$ under the assumptions mentioned in the statement. As the equalities $2 \nu_{n}\left(\varphi_{M_{0}}\right) \nu_{n}\left(\varphi_{F_{1}}\right)+\delta \nu_{n}\left(\varphi_{F_{1}}\right)^{2}=$ $\operatorname{vol}\left(\nu_{n}\right)^{-1}$ and $D^{2}=\operatorname{vol}(D)$ are satisfied, one gets

$$
\begin{aligned}
\frac{\operatorname{vol}(D)}{\operatorname{vol}\left(\nu_{n}\right)} & =\left(2 a b \delta+b^{2} \delta^{2}\right) \nu_{n}\left(\varphi_{F_{1}}\right)^{2}+2 b(a+b \delta) \nu_{n}\left(\varphi_{F_{1}}\right) \nu_{n}\left(\varphi_{M_{0}}\right)+2 a b \nu_{n}\left(\varphi_{F_{1}}\right) \nu_{n}\left(\varphi_{M_{0}}\right) \\
& =(a+b \delta)^{2} \nu_{n}\left(\varphi_{F_{1}}\right)^{2}+2 b(a+b \delta) \nu_{n}\left(\varphi_{F_{1}}\right) \nu_{n}\left(\varphi_{M_{0}}\right)+b^{2} \nu_{n}\left(\varphi_{M_{0}}\right)^{2} \\
& =\hat{\mu}_{D}\left(\nu_{n}\right)^{2}
\end{aligned}
$$

where the second equality is obtained as consequence of the condition $\left(a \nu_{n}\left(\varphi_{F_{1}}\right)-\right.$ $\left.b \nu_{n}\left(\varphi_{M_{0}}\right)\right)^{2}=0$, which holds by hypothesis. This shows that $\nu_{n}$ is minimal with respect to $D$.

Conversely suppose that $\nu_{n}$ is minimal with respect to $D$. Theorem 3.2.1 shows that

$$
\left((a+b \delta) \nu_{n}\left(\varphi_{F_{1}}\right)+b \nu_{n}\left(\varphi_{M_{0}}\right)\right)^{2}=b(2 a+\delta b) \operatorname{vol}\left(\nu_{n}\right)^{-1}
$$


In addition, it holds that

$$
\begin{aligned}
\left((a+b \delta) \nu_{n}\left(\varphi_{F_{1}}\right)+b \nu_{n}\left(\varphi_{M_{0}}\right)\right)^{2}= & a^{2} \nu_{n}\left(\varphi_{F_{1}}\right)^{2}+b^{2} \nu_{n}\left(\varphi_{M_{0}}\right)^{2}+b(2 a+b \delta) \delta \nu_{n}\left(\varphi_{F_{1}}\right)^{2} \\
& +b(a+\delta b)\left(2 \nu_{n}\left(\varphi_{F_{1}}\right) \nu_{n}\left(\varphi_{M_{0}}\right)\right) \\
= & \left(a \nu_{n}\left(\varphi_{F_{1}}\right)-b \nu_{n}\left(\varphi_{M_{0}}\right)\right)^{2} \\
& +b(2 a+\delta b)\left(2 \nu_{n}\left(\varphi_{F_{1}}\right) \nu_{n}\left(\varphi_{M_{0}}\right)+\delta \nu_{n}\left(\varphi_{F_{1}}\right)^{2}\right)
\end{aligned}
$$

which, together with Equality (3.4), gives rise to

$\left(a \nu_{n}\left(\varphi_{F_{1}}\right)-b \nu_{n}\left(\varphi_{M_{0}}\right)\right)^{2}+b(2 a+\delta b)\left(2 \nu_{n}\left(\varphi_{F_{1}}\right) \nu_{n}\left(\varphi_{M_{0}}\right)+\delta \nu_{n}\left(\varphi_{F_{1}}\right)^{2}-\operatorname{vol}\left(\nu_{n}\right)^{-1}\right)=0$.

Both summands in the above expression are not negative and so they must vanish. This concludes the proof.

Corollary 3.2.4. Let $\nu_{n}$ be an NPI divisorial valuation of $\mathbb{F}_{\delta}$. Then $\nu_{n}$ is nonminimal with respect to any big divisor $D$ on $\mathbb{F}_{\delta}$ whenever some of the following conditions holds:

(a) $\nu_{n}$ is special and $2 \nu_{n}\left(\varphi_{M_{0}}\right) \nu_{n}\left(\varphi_{F_{1}}\right)+\delta \nu_{n}\left(\varphi_{F_{1}}\right)^{2}>\operatorname{vol}\left(\nu_{n}\right)^{-1}$.

(b) $\nu_{n}$ is non-special and $2 \nu_{n}\left(\varphi_{M_{1}}\right) \nu_{n}\left(\varphi_{F_{1}}\right)-\delta \nu_{n}\left(\varphi_{F_{1}}\right)^{2}>\operatorname{vol}\left(\nu_{n}\right)^{-1}$.

Proof. Let us first prove (a). It suffices to check that any big divisor $D \sim a F+b M$ satisfies

$$
\hat{\mu}_{D}\left(\nu_{n}\right)^{2} / P_{D}^{2}>\bar{\beta}_{g+1}\left(\nu_{n}\right),
$$

where $P_{D}$ is the positive part of the Zariski decomposition of $D$ (see Remark 1.6.8). Define the map $q_{1}:(-\delta, \infty) \rightarrow \mathbb{R}_{>0}$ as

$$
q_{1}(x):=\left\{\begin{array}{l}
\frac{\left((x+\delta) \nu_{n}\left(\varphi_{F_{1}}\right)+\nu_{n}\left(\varphi_{M_{0}}\right)\right)^{2}}{((1 / \delta) x+1)^{2} \delta} \text { if } x \in(-\delta, 0), \\
\frac{\left((x+\delta) \nu_{n}\left(\varphi_{F_{1}}\right)+\nu_{n}\left(\varphi_{M_{0}}\right)\right)^{2}}{2 x+\delta} \text { if } x \in[0, \infty) .
\end{array}\right.
$$

It follows easily that $q_{1}$ has an absolute minimum at the point $\left(x_{1}, q_{1}\left(x_{1}\right)\right)$, where

$$
x_{1}=\frac{\nu_{n}\left(\varphi_{M_{0}}\right)}{\nu_{n}\left(\varphi_{F_{1}}\right)} \text { and } q_{1}\left(x_{1}\right)=2 \nu_{n}\left(\varphi_{M_{0}}\right) \nu_{n}\left(\varphi_{F_{1}}\right)+\nu_{n}\left(\varphi_{F_{1}}\right)^{2} \delta \text {. }
$$

The equality $q_{1}(a / b)=\hat{\mu}_{D}\left(\nu_{n}\right)^{2} / P_{D}^{2}$, the assumption

$$
2 \nu_{n}\left(\varphi_{M_{0}}\right) \nu_{n}\left(\varphi_{F_{1}}\right)+\delta \nu_{n}\left(\varphi_{F_{1}}\right)^{2}>\operatorname{vol}\left(\nu_{n}\right)^{-1}
$$

and Theorem 3.2.1 complete the proof.

Finally, we can proceed analogously as above to prove (b). Here we have to consider the map $q_{2}:(-\delta, \infty) \rightarrow \mathbb{R}_{>0}$,

$$
q_{2}(x):=\left\{\begin{array}{l}
\frac{\left(\nu_{n}\left(\varphi_{F_{1}}\right) x+\nu_{n}\left(\varphi_{M_{1}}\right)\right)^{2}}{((1 / \delta) x+1)^{2} \delta} \text { if } x \in(-\delta, 0), \\
\frac{\left(\nu_{n}\left(\varphi_{F_{1}}\right) x+\nu_{n}\left(\varphi_{M_{1}}\right)\right)^{2}}{2 x+\delta} \text { if } x \in[0, \infty),
\end{array}\right.
$$


instead of $q_{1}$, which has an absolute minimum at the point $\left(x_{2}, q_{2}\left(x_{2}\right)\right)$, where

$$
x_{2}=\frac{\nu_{n}\left(\varphi_{M_{1}}\right)-\delta \nu_{n}\left(\varphi_{F_{1}}\right)}{\nu_{n}\left(\varphi_{F_{1}}\right)} \text { and } q_{2}\left(x_{2}\right)=2 \nu_{n}\left(\varphi_{M_{1}}\right) \nu_{n}\left(\varphi_{F_{1}}\right)-\delta \nu_{n}\left(\varphi_{F_{1}}\right)^{2}
$$

\subsection{Newton-Okounkov bodies of non-positive at infinity valuations}

Newton-Okounkov bodies are convex sets which provide interesting geometric information [86, 74, 15]. However, giving an explicit description of them is very difficult. As we have seen in Subsection 1.5.2, these bodies can be described; however the involved objects for that description are also very hard to compute. In this section we give a much more simple and explicit description of the Newton-Okounkov bodies of big divisors on a surface $Z_{0}$, which is the projective plane $\mathbb{P}^{2}:=\mathbb{P}_{\mathbb{C}}^{2}$ or a Hirzebruch surface $\mathbb{F}_{\delta}$ over $\mathbb{C}, \delta \geq 0$, with respect to flags defined by exceptional divisors associated to NPI divisorial valuations.

Let $E_{r}$ be the last exceptional divisor created by a finite simple sequence of blowups

$$
\pi: Z:=Z_{r} \stackrel{\pi_{r}}{\longrightarrow} Z_{r-1} \rightarrow \ldots \rightarrow Z_{1} \stackrel{\pi_{1}}{\longrightarrow} Z_{0}
$$

and $p_{i+1}, 0 \leq i \leq r-1$, the closed point of $Z_{i}$ where the blowup $\pi_{i+1}: Z_{i+1} \rightarrow Z_{i}$ is centered. Denote by $E_{\bullet}$ the flag of $Z$ (see Subsection 1.5.2) defined as

$$
E_{\bullet}:=\left\{Z=Z_{r} \supset E_{r} \supset\left\{p_{r+1}\right\}\right\}
$$

where the closed point $p_{r+1} \in E_{r}$ is the center of $E_{\bullet}$. The point $p_{r+1}$ could belong to another exceptional divisor. In this case, this divisor is denoted by $E_{\eta}$, that is, $\eta$ is a positive integer $\eta<r$ such that $p_{r+1} \in E_{\eta} \cap E_{r}$.

As we have mentioned in Subsection 1.5.2, a flag of a smooth surface comes with a discrete valuation of rank 2 ; in fact, they are equivalent objects. In this chapter, following [65, Section 3.2] and our Section 1.3, the discrete valuation $\nu:=$ $\nu_{E_{\bullet}}$ attached to $E_{\bullet}$ corresponds with an exceptional curve valuation whose value group is $\mathbb{Z}^{2}$ and $\nu\left(\mathfrak{m}_{r}\right)=(1,0)$ and $\nu\left(\mathfrak{m}_{r+1}\right)=(0,1)$, up to equivalence. Recall that $\nu$ can be computed as follows: $\nu(f)=\left(v_{1}(f), v_{2}(f)\right)$, where

$$
v_{1}(f):=\nu_{r}(f) \text { and } v_{2}(f):=\nu_{\eta}(f)+\sum_{p_{i} \rightarrow p_{r}} \operatorname{mult}_{p_{i}}(f), \text { for } f \in \mathcal{O}_{Z_{0}, p},
$$

$\nu_{r}$ (respectively, $\nu_{\eta}$ ) being the divisorial valuation defined by $E_{r}$ (respectively, $E_{\eta}$ ).

Set $\mathcal{C}_{\nu}=\left\{p_{i}\right\}_{i \geq 1}$ the configuration of infinitely near points of $\nu$, where $p_{i} \rightarrow p_{r}$ for all $i>r$, and $\mathcal{C}_{\nu_{r}}=\left\{p_{i}\right\}_{i=1}^{r}$ the configuration of infinitely near points of $\nu_{r}$. Write $\Gamma_{\nu}$ (respectively, $\Gamma_{\nu_{r}}$ ) the dual graph of $\nu$ (respectively, $\nu_{r}$ ). We consider the following 
useful ordering on the set of vertices of $\Gamma_{\nu_{r}}$ (Section 1.3): given two vertices $\alpha$ and $\beta$, we say that $\alpha \preccurlyeq \beta$ if there exists a simple path on the graph $\Gamma_{\nu_{r}}$ from 1 to $\beta$ passing through $\alpha$.

Denote by $\left\{\bar{\beta}_{i}\left(\nu_{n}\right)\right\}_{i=0}^{g+1}$ the sequence of maximal contact values of the divisorial valuation $\nu_{n}$ for $n=r, \eta$. Write $S_{\nu}$ the semigroup of values of $\nu$. It is generated by $\left\{\bar{\beta}_{i}(\nu)\right\}_{i=0}^{g^{*}+1}$, where $\bar{\beta}_{i}(\nu)=\left(\bar{\beta}_{i}\left(\nu_{r}\right), \bar{\beta}_{i}\left(\nu_{\eta}\right)\right)$ (respectively, $\bar{\beta}_{i}(\nu)=\left(\bar{\beta}_{i}\left(\nu_{r}\right), 0\right)$ and $\left.\bar{\beta}_{g^{*}+1}(\nu)=\left(\bar{\beta}_{g^{*}+1}\left(\nu_{r}\right), 1\right)\right)$ if the point $p_{r+1}$ is satellite (respectively, free) (see [65]). Notice that it holds that $g=g^{*}+1$ if $p_{r+1}$ and $p_{r}$ are satellite points. Otherwise, $g=g^{*}$.

The distinction between special and non-special valuations and the definition of minimal valuation given for divisorial valuations can be easily extended to the exceptional curve case.

Definition 3.3.1. Let $\nu$ be an exceptional curve valuation of $Z_{0}$. We say that $\nu$ is special when $Z_{0}=\mathbb{F}_{\delta}, \delta \geq 0$, and its first component $\nu_{r}$ is special, and is non-special if $Z_{0}=\mathbb{F}_{\delta}, \delta>0$, and its first component $\nu_{r}$ is non-special. Likewise, the valuation $\nu$ is called to be non-positive at infinity (NPI) when its first component $\nu_{r}$ is NPI.

Definition 3.3.2. Let $D$ be a big divisor on a surface $Z_{0}$. An exceptional curve valuation of $Z_{0}$ is said to be minimal with respect to $D$ if its first component $\nu_{r}$ is minimal with respect to $D$.

Notice that, in the above definition, is enough to say minimal when $Z_{0}=\mathbb{P}^{2}$ by Proposition 3.1.3.

The aim of this section is to explicitly compute Newton-Okounkov bodies of divisors $D^{*}$ with respect to $\nu:=\nu_{E_{\bullet}}$, where $\nu$ is a exceptional curve valuation of $Z_{0}, E_{\text {• }}$ is a flag as (3.6), and $D^{*}$ is the total transform of a big divisor $D$ on $Z_{0}$. Under the above conditions, $H^{0}\left(Z_{0}, \mathcal{O}_{Z_{0}}(D)\right) \cong H^{0}\left(Z, \mathcal{O}_{Z}\left(D^{*}\right)\right)$ holds and, without restriction of generality, we can use the following definition about Newton-Okounkov bodies.

Definition 3.3.3. Let $\nu$ be an exceptional curve valuation of $Z_{0}$ and $D$ a big divisor on $Z_{0}$. The Newton-Okounkov body of $D$ with respect to $\nu$ is defined as

$$
\Delta_{\nu}(D):=\overline{\bigcup_{m \geq 1}\left\{\frac{\nu(f)}{m} \mid f \in H^{0}\left(Z_{0}, m D\right) \backslash\{0\}\right\}}
$$

where the upper line means closed convex hull in $\mathbb{R}^{2}$.

Remark 3.3.4. When $Z_{0}=\mathbb{P}^{2}$, it suffices to study the case where $D$ is a projective line since $\Delta_{\nu}(d D)=d \cdot \Delta_{\nu}(D)$ for all integer $d>0$.

Set $\mathfrak{C}(\nu)$ the convex cone of $\mathbb{R}^{2}$ generated by the semigroup of values $S_{\nu}$ of $\nu$ and $\mathfrak{H}_{D}(\nu)$ the half-plane $\left\{(x, y) \in \mathbb{R}^{2} \mid x \leq \hat{\mu}_{D}\left(\nu_{r}\right)\right\}$. We explicitly describe the set $\mathfrak{C}(\nu) \cap \mathfrak{H}_{D}(\nu)$ in the following result. 
Proposition 3.3.5. Keep the notation introduced before. The Newton-Okounkov body $\Delta_{\nu}(D)$ of $D$ with respect to $\nu$ is contained in the triangle $\mathfrak{C}(\nu) \cap \mathfrak{H}_{D}(\nu)$ whose vertices are

$$
(0,0), \quad\left(\hat{\mu}_{D}\left(\nu_{r}\right), \frac{\hat{\mu}_{D}\left(\nu_{r}\right) \bar{\beta}_{0}\left(\nu_{\eta}\right)}{\bar{\beta}_{0}\left(\nu_{r}\right)}\right) \quad \text { and } \quad\left(\hat{\mu}_{D}\left(\nu_{r}\right), \frac{\hat{\mu}_{D}\left(\nu_{r}\right) \bar{\beta}_{g^{*}+1}\left(\nu_{\eta}\right)}{\bar{\beta}_{g^{*}+1}\left(\nu_{r}\right)}\right)
$$

if $p_{r+1} \in E_{\eta} \cap E_{r}$ (with $\left.\eta \neq r\right)$; and

$$
(0,0), \quad\left(\hat{\mu}_{D}\left(\nu_{r}\right), 0\right) \quad \text { and } \quad\left(\hat{\mu}_{D}\left(\nu_{r}\right), \frac{\hat{\mu}_{D}\left(\nu_{r}\right)}{\bar{\beta}_{g+1}\left(\nu_{r}\right)}\right),
$$

otherwise.

Proof. To start, we suppose that $p_{r+1}$ is the satellite point $E_{\eta} \cap E_{r}$. Following Subsection 1.3.1 and Corollary 1.3.7, the $j$ th maximal contact value of $\nu, 0 \leq j \leq g^{*}$, is $\bar{\beta}_{j}(\nu)=\left(\bar{\beta}_{j}\left(\nu_{r}\right), \bar{\beta}_{j}\left(\nu_{\eta}\right)\right)$. All these values belong to the line passing through the origin with slope $\bar{\beta}_{0}\left(\nu_{\eta}\right) / \bar{\beta}_{0}\left(\nu_{r}\right)$. This line gives a ray of the cone $\mathfrak{C}(\nu)$ which is defined by another line going through the origin with slope $\bar{\beta}_{g^{*}+1}\left(\nu_{\eta}\right) / \bar{\beta}_{g^{*}+1}\left(\nu_{r}\right)$. Both lines together with the line $x=\hat{\mu}_{D}\left(\nu_{r}\right)$ determine the triangle $\mathfrak{C}(\nu) \cap \mathfrak{H}_{D}(\nu)$ giving rise to the displayed vertices. Finally, Equality (3.1) completes the proof of (a).

Let us assume that $p_{r+1}$ is a free point. It holds that $\bar{\beta}_{j}(\nu)=\left(\bar{\beta}_{j}\left(\nu_{r}\right), 0\right)$ for $j \in\left\{0,1, \ldots, g^{*}\right\}$ and $\bar{\beta}_{g^{*}+1}(\nu)=\left(\bar{\beta}_{g+1}\left(\nu_{r}\right), 1\right)$, and consequently one obtains the triangle $\mathfrak{C}(\nu) \cap \mathfrak{H}_{D}(\nu)$. Finally, by Equality (3.1), we conclude the proof.

Next, we provide a description of the Newton-Okounkov bodies with respect to a minimal exceptional curve valuation of $Z_{0}$. Before stating the result, we show a useful lemma.

Lemma 3.3.6. Keep the notation introduced before. Assume that $p_{r+1}$ is the satellite point $E_{\eta} \cap E_{r}, \eta \neq r$. Then,

(a) It holds that

$$
\bar{\beta}_{g+1}\left(\nu_{r}\right)=\left|\frac{\bar{\beta}_{g^{*}+1}\left(\nu_{\eta}\right)}{\bar{\beta}_{g^{*}+1}\left(\nu_{r}\right)}-\frac{\bar{\beta}_{0}\left(\nu_{\eta}\right)}{\bar{\beta}_{0}\left(\nu_{r}\right)}\right|^{-1} .
$$

(b) If $\eta \preccurlyeq r$, then

$$
\nu_{r}\left(\varphi_{\eta}\right)=\bar{\beta}_{g+1}\left(\nu_{r}\right) \cdot \frac{\bar{\beta}_{g^{*}+1}\left(\nu_{\eta}\right)}{\bar{\beta}_{g^{*}+1}\left(\nu_{r}\right)} \text { and } \nu_{r}\left(\varphi_{\eta}\right)+1=\bar{\beta}_{g+1}\left(\nu_{r}\right) \cdot \frac{\bar{\beta}_{0}\left(\nu_{\eta}\right)}{\bar{\beta}_{0}\left(\nu_{r}\right)} \text {. }
$$

(c) If $\eta \npreceq r$, then

$$
\nu_{r}\left(\varphi_{\eta}\right)=\bar{\beta}_{g+1}\left(\nu_{r}\right) \cdot{\overline{\beta_{0}}\left(\nu_{\eta}\right)}_{\bar{\beta}_{0}\left(\nu_{r}\right)} \text { and } \nu_{r}\left(\varphi_{\eta}\right)+1=\bar{\beta}_{g+1}\left(\nu_{r}\right) \cdot \frac{\bar{\beta}_{g^{*}+1}\left(\nu_{\eta}\right)}{\bar{\beta}_{g^{*}+1}\left(\nu_{r}\right)} .
$$


Proof. The first item is proved in [65, Lemma 3.9]. We are going to show Item (b). We can distinguish three cases:

Case 1: Assume that $p_{r}$ is a free point. That is, $\eta=r-1, \eta \preccurlyeq r$ and $g=g^{*}$. By Corollary 1.3.7,

$$
\nu_{r}\left(\varphi_{\eta}\right)+1=\bar{\beta}_{g+1}\left(\nu_{r}\right)=\bar{\beta}_{g+1}\left(\nu_{r}\right) \cdot \frac{\bar{\beta}_{0}\left(\nu_{\eta}\right)}{\bar{\beta}_{0}\left(\nu_{r}\right)} .
$$

Finally, it holds that

$$
\nu_{r}\left(\varphi_{\eta}\right)=\bar{\beta}_{g+1}\left(\nu_{\eta}\right)=\bar{\beta}_{g+1}\left(\nu_{\eta}\right) \cdot \frac{\bar{\beta}_{g+1}\left(\nu_{r}\right)}{\bar{\beta}_{g+1}\left(\nu_{r}\right)}=\bar{\beta}_{g+1}\left(\nu_{r}\right) \cdot \frac{\bar{\beta}_{g^{*}+1}\left(\nu_{\eta}\right)}{\bar{\beta}_{g^{*}+1}\left(\nu_{r}\right)},
$$

which proves the result in this case.

Case 2: Let us show the case when $p_{r}$ is a satellite point, $p_{\eta}$ is a free point and $\eta<\ell_{g}$. That is, $\eta=\ell_{g}-1, \eta \preccurlyeq r$ and $g=g^{*}+1$. In addition, it holds that $\beta_{i}^{\prime}\left(\nu_{r}\right)=\beta_{i}^{\prime}\left(\nu_{\eta}\right)$ for $0 \leq i \leq g-1$ and $\nu_{r}\left(\varphi_{\eta}\right)=e_{g-1}\left(\nu_{r}\right) \nu_{\eta}\left(\varphi_{\eta}\right)$. As a consequence, it holds that

$$
\nu_{r}\left(\varphi_{\eta}\right)=e_{g-1}\left(\nu_{r}\right) \nu_{\eta}\left(\varphi_{\eta}\right)=\bar{\beta}_{g+1}\left(\nu_{r}\right) \cdot \frac{\bar{\beta}_{g}\left(\nu_{\eta}\right)}{\bar{\beta}_{g}\left(\nu_{r}\right)}=\bar{\beta}_{g+1}\left(\nu_{r}\right) \cdot \frac{\bar{\beta}_{g^{*}+1}\left(\nu_{\eta}\right)}{\bar{\beta}_{g^{*}+1}\left(\nu_{r}\right)}
$$

and

$$
\nu_{r}\left(\varphi_{\eta}\right)+1=\bar{\beta}_{g}\left(\nu_{r}\right)=\frac{\bar{\beta}_{g+1}\left(\nu_{r}\right)}{e_{g-1}\left(\nu_{r}\right)}=\bar{\beta}_{g+1}\left(\nu_{r}\right) \cdot \frac{\bar{\beta}_{0}\left(\nu_{\eta}\right)}{\bar{\beta}_{0}\left(\nu_{r}\right)}
$$

by Corollary 1.3 .7 and the equality $\bar{\beta}_{g+1}\left(\nu_{r}\right)=e_{g-1}\left(\nu_{r}\right) \bar{\beta}_{g}\left(\nu_{r}\right)$.

Case 3: Suppose now that $\eta \preccurlyeq r$ and neither Case 1 nor Case 2 hold. Thus, $g=g^{*}+1$ holds since $p_{r}$ and $p_{r+1}$ are satellite points. In this situation, one has

$$
\left.e_{g-1}\left(\nu_{\eta}\right) \bar{\beta}_{g}\left(\nu_{r}\right)>e_{g-1}\left(\nu_{r}\right) \bar{\beta}_{g}\left(\nu_{\eta}\right)\right)=\nu_{r}\left(\varphi_{\eta}\right) \text {, }
$$

by [65, Proposition 2.5]. Thus,

$$
\nu_{r}\left(\varphi_{\eta}\right)=\bar{\beta}_{g+1}\left(\nu_{r}\right) \cdot \frac{\bar{\beta}_{g}\left(\nu_{\eta}\right)}{\bar{\beta}_{g}\left(\nu_{r}\right)}=\bar{\beta}_{g+1}\left(\nu_{r}\right) \cdot \frac{\bar{\beta}_{g^{*}+1}\left(\nu_{\eta}\right)}{\bar{\beta}_{g^{*}+1}\left(\nu_{r}\right)} .
$$

Moreover, by [65, Lemma 3.9], $e_{g-1}\left(\nu_{\eta}\right) \bar{\beta}_{g}\left(\nu_{r}\right)-e_{g-1}\left(\nu_{r}\right) \bar{\beta}_{g}\left(\nu_{\eta}\right)=1$ and then, by Corollary 1.3.7,

$$
\nu_{r}\left(\varphi_{\eta}\right)+1=e_{g-1}\left(\nu_{\eta}\right) \bar{\beta}_{g}\left(\nu_{r}\right)=e_{g-1}\left(\nu_{r}\right) \bar{\beta}_{g}\left(\nu_{r}\right) \cdot \frac{\bar{\beta}_{0}\left(\nu_{\eta}\right)}{\bar{\beta}_{0}\left(\nu_{r}\right)}=\bar{\beta}_{g+1}\left(\nu_{r}\right) \cdot \frac{\bar{\beta}_{0}\left(\nu_{\eta}\right)}{\bar{\beta}_{0}\left(\nu_{r}\right)},
$$

which completes the proof of (b).

Finally, Item (c) can be proved arguing as in the proof of Case 3 of (b).

Theorem 3.3.7. Let $\nu$ be an exceptional curve valuation of $Z_{0}$ and set $D$ a big divisor on $Z_{0}$. Then, the Newton-Okounkov body $\Delta_{\nu}(D)$ of $D$ with respect to $\nu$ coincides with the triangle $\mathfrak{C}(\nu) \cap \mathfrak{H}_{D}(\nu)$ if and only if $\nu$ is minimal with respect to $D$. 
Proof. We start by recalling that the triangle $\mathfrak{C}(\nu) \cap \mathfrak{H}_{D}(\nu)$ contains the NewtonOkounkov body $\Delta_{\nu}(D)$ by Proposition 3.3.5. In addition, the area of this triangle is

$$
\frac{\hat{\mu}_{D}\left(\nu_{r}\right)^{2}}{2}\left|\frac{\bar{\beta}_{g^{*}+1}\left(\nu_{\eta}\right)}{\bar{\beta}_{g^{*}+1}\left(\nu_{r}\right)}-\frac{\bar{\beta}_{0}\left(\nu_{\eta}\right)}{\bar{\beta}_{0}\left(\nu_{r}\right)}\right|\left(\text { respectively, } \frac{\hat{\mu}_{D}\left(\nu_{r}\right)^{2}}{2 \bar{\beta}_{g+1}\left(\nu_{r}\right)}\right)
$$

when $p_{r+1}$ is a satellite point (respectively, $p_{r+1}$ is a free point). By Lemma 3.3.6, the area of the triangle $\mathfrak{C}(\nu) \cap \mathfrak{H}_{D}(\nu)$ in the satellite case becomes $\hat{\mu}_{D}\left(\nu_{r}\right)^{2} / 2 \bar{\beta}_{g+1}\left(\nu_{r}\right)$ and then, in both cases, the triangle $\mathfrak{C}(\nu) \cap \mathfrak{H}_{D}(\nu)$ has the same area. From Subsection 1.5.2 and (3.2), one can deduce that

$$
\frac{\hat{\mu}_{D}\left(\nu_{r}\right)^{2}}{2 \bar{\beta}_{g+1}\left(\nu_{r}\right)} \geq \frac{\operatorname{vol}(D)}{2}=\operatorname{vol}_{\mathbb{R}^{2}}\left(\Delta_{\nu}(D)\right)
$$

and therefore the triangle $\mathfrak{C}(\nu) \cap \mathfrak{H}_{D}(\nu)$ and the body $\Delta_{\nu}$ coincide if and only if they have the same area, or equivalently, the valuation $\nu$ is minimal with respect to D.

Remark 3.3.8. When $Z_{0}=\mathbb{P}^{2}$, the above theorem has been proved for any big divisor $D$ on $\mathbb{P}^{2}$. Notice that the Newton-Okounkov bodies $\Delta_{\nu}(D)$ described in the minimal case satisfy the homothetic property by Lemma 3.1.2.

Notice that, if we consider NPI exceptional curve valuations, then we can compute explicitly the values $\hat{\mu}_{D}\left(\nu_{r}\right)$ and therefore Newton-Okounkov bodies in the minimal case. Let us show and example:

Example 3.3.9. Let $p$ be a special point of the Hirzebruch surface $\mathbb{F}_{2}$ and $\nu_{r}$ a special divisorial valuation centered at $\mathcal{O}_{\mathbb{F}_{2}, p}$ whose maximal contact values are $\left\{\bar{\beta}_{i}\left(\nu_{r}\right)\right\}_{i=0}^{4}=\{12,18,117,239,720\}$. Set $\mathcal{C}_{\nu_{r}}=\left\{p_{i}\right\}_{i=0}^{25}$ (where $p=p_{1}$ ) its configuration of infinitely near points, $F_{1}$ the fiber containing $p$ and $M_{0}$ the special section whose strict transform goes through $p_{2}$. Therefore

$$
\nu_{r}\left(\varphi_{F_{1}}\right)=12, \nu_{r}\left(\varphi_{M_{0}}\right)=18 \text { and } 2 \nu_{r}\left(\varphi_{M_{0}}\right) \nu_{r}\left(\varphi_{F_{1}}\right)+\delta \nu_{r}\left(\varphi_{F_{1}}\right)^{2}=720 .
$$

Consider the divisor $D=18 F+12 M$. By Theorem 3.2.1 and Corollary 3.2.3, the value $\hat{\mu}_{D}\left(\nu_{r}\right)$ is equal to 720 and then $\nu_{r}$ is minimal with respect to $D$.

Let $\nu=\nu_{E_{\bullet}}$ be the valuation defined by the flag $E_{\bullet}=\left\{Z_{25} \supset E_{25} \supset\left\{p_{26}\right\}\right\}$, where $p_{26} \in E_{25} \cap E_{24}$, and whose first component is the previous valuation $\nu_{r}$. The semigroup of values $S_{\nu}$ of $\nu$ is generated by

$$
\left\{\bar{\beta}_{j}(\nu)\right\}_{i=0}^{4}=\{(12,12),(18,18),(117,117),(239,239),(720,719)\}
$$

and, by Theorem 3.3.7, the coordinates of the Newton-Okounkov body $\Delta_{\nu}(D)$ (Figure 3.1(a)) of $D$ with respect to $\nu$ are

$$
\mathbf{0}=(0,0), Q_{1}=(720,720) \text { and } Q_{2}=(720,719) .
$$


If now we assume that $p_{26}$ is a free point, we get another example. Here, the semigroup of values $S_{\nu}$ of $\nu$ is generated by

$$
\left\{\bar{\beta}_{j}(\nu)\right\}_{i=0}^{4}=\{(12,0),(18,0),(117,0),(239,0),(720,1)\}
$$

and the coordinates of the vertices of $\Delta_{\nu}(D)$ (Figure 3.1(b)) are

$$
\mathbf{0}=(0,0), Q_{1}=(720,0) \text { and } Q_{2}=(720,1) .
$$

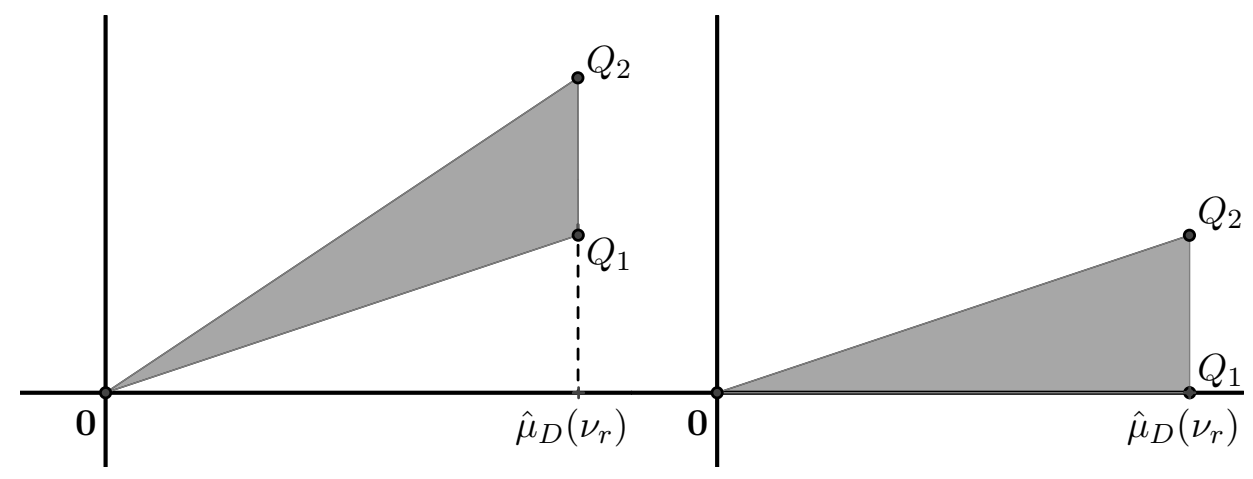

(a) The point $p_{r+1}$ is satellite.

(b) The point $p_{r+1}$ is free.

Figure 3.1: $\Delta_{\nu}(18 F+12 M)$ in Example 3.3.9.

Explicitly computing Newton-Okounkov bodies with respect to non-minimal valuation is a hard task. Whenever $Z_{0}=\mathbb{P}^{2}$, an explicit description of the vertices of these bodies can be found in [65, Theorems 3.12 and 3.14]. When considering a non-positive at infinity exceptional curve valuation $\nu$ of $\mathbb{P}^{2}$, the Newton-Okounkov body $\Delta_{\nu}\left(E_{0}\right)$ of a general projective line $E_{0}$ can be completely computed as we show in the next result, which will be proved later. The proof is a consequence of further results (see Corollary 3.3.29).

Theorem 3.3.10. Let $E_{\bullet}=\left\{Z=Z_{r} \supset E_{r} \supset\left\{p_{r+1}\right\}\right\}$ be a flag and $\nu=\nu_{E_{\bullet}}$ its attached exceptional curve valuation. Assume that the first component $\nu_{r}$ of $\nu$ is an NPI divisorial valuation of $\mathbb{P}^{2}$. Denote by $\bar{\beta}(\nu)_{i=0}^{g^{*}+1}$ (respectively, $\bar{\beta}\left(\nu_{r}\right)_{i=0}^{g^{*}+1}$ ) the sequence of maximal contact values of $\nu$ (respectively, $\left.\nu_{r}\right)$. Consider a general projective line $E_{0}$ and the projective line at infinity $L$ as in Subsection 2.1. Then the Newton-Okounkov body of $E_{0}$ with respect to $\nu=\nu_{E_{\bullet}}$ is a triangle whose vertices have the following coordinates:

(a)

$$
\mathbf{0}=(0,0), Q_{1}=\left(\frac{\bar{\beta}_{g+1}\left(\nu_{r}\right)}{\nu_{r}\left(\varphi_{L}\right)}, 0\right) \text { and } Q_{2}=\left(\nu_{r}\left(\varphi_{L}\right), 1\right),
$$

when $p_{r+1}$ is a free point and $\nu\left(\varphi_{L}\right)=\bar{\beta}_{1}(\nu)$. 
(b)

$$
\mathbf{0}=(0,0), Q_{1}=\left(\frac{\bar{\beta}_{g+1}\left(\nu_{r}\right)}{\nu_{r}\left(\varphi_{L}\right)}, \frac{1}{\nu_{r}\left(\varphi_{L}\right)}\right) \text { and } Q_{2}=\left(\nu_{r}\left(\varphi_{L}\right), 0\right),
$$

when $p_{r+1}$ is a free point and $\nu\left(\varphi_{L}\right) \neq \bar{\beta}_{1}(\nu)$.

(c)

$$
\mathbf{0}=(0,0), Q_{1}=\left(\frac{\bar{\beta}_{g+1}\left(\nu_{r}\right)}{\nu_{r}\left(\varphi_{L}\right)}, \frac{\nu_{r}\left(\varphi_{\eta}\right)}{\nu_{r}\left(\varphi_{L}\right)}\right) \text { and } Q_{2}=\left(\nu_{r}\left(\varphi_{L}\right), \nu_{\eta}\left(\varphi_{L}\right)\right),
$$

when $p_{r+1} \in E_{\eta} \cap E_{r}(\eta \neq r), \eta \preccurlyeq r$ and, either $g^{*}>0$, or $g^{*}=0$ and $\nu\left(\varphi_{L}\right) \neq \bar{\beta}_{1}(\nu)$. The latter points also describe the Newton-Okounkov body of $E_{0}$ if $p_{r+1}$ is the satellite point $E_{\eta} \cap E_{r}(\eta \neq r), \eta \npreceq r, g^{*}=0$ and $\nu\left(\varphi_{L}\right)=\bar{\beta}_{1}(\nu)$.

(d)

$$
\mathbf{0}=(0,0), Q_{1}=\left(\frac{\bar{\beta}_{g+1}\left(\nu_{r}\right)}{\nu_{r}\left(\varphi_{L}\right)}, \frac{\nu_{r}\left(\varphi_{\eta}\right)+1}{\nu_{r}\left(\varphi_{L}\right)}\right) \text { and } Q_{2}=\left(\nu_{r}\left(\varphi_{L}\right), \nu_{\eta}\left(\varphi_{L}\right)\right) \text {, }
$$

when $p_{r+1} \in E_{\eta} \cap E_{r}(\eta \neq r), \eta \npreceq r$ and, either $g^{*}>0$, or $g^{*}=0$ and $\nu\left(\varphi_{L}\right) \neq \bar{\beta}_{1}(\nu)$. The latter points also describe the Newton-Okounkov body of $E_{0}$ if $p_{r+1}$ is the satellite point $E_{\eta} \cap E_{r}(\eta \neq r), \eta \preccurlyeq r, g^{*}=0$ and $\nu\left(\varphi_{L}\right)=\bar{\beta}_{1}(\nu)$.

Let us see an example which corresponds to Theorem 3.3.10.

Example 3.3.11. Let $\nu_{n}$ be a divisorial valuation of $\mathbb{P}^{2}$ whose configuration of infinitely near points is $\mathcal{C}_{\nu_{n}}=\left\{p_{i}\right\}_{i=1}^{17}$ and whose sequence of maximal contact values is $\{8,20,63,256\}$. Firstly, assume that the strict transforms of the projective line $L$ pass through $p_{1}$ and $p_{2}$. Then, by Remark 3.1.7,

$$
\hat{\mu}\left(\nu_{n}\right)=\nu_{n}\left(\varphi_{L}\right)=16 \text { and } \hat{\mu}\left(\nu_{n}\right)^{2}=256=\bar{\beta}_{g+1}\left(\nu_{n}\right) .
$$

Consequently, the valuation $\nu_{n}$ is non-positive at infinity and minimal.

Set $\nu:=\nu_{E}$. the exceptional curve valuation of $\mathbb{P}^{2}$ which is defined by the flag $\left\{Z_{17} \supset E_{17} \supset\left\{p_{18}\right\}\right\}$. Its first coordinate is the above divisorial valuation $\nu_{n}$. If we assume that $p_{18}$ is satellite, then $p_{18} \in E_{16} \cap E_{17}$ and the Newton-Okounkov body $\Delta_{\nu}\left(E_{0}\right)$ of a general projective line $E_{0}$ with respect to $\nu$ is a triangle whose coordinates are

$$
\mathbf{0}=(0,0),(16,16) \text { and }\left(16, \frac{255}{16}\right) .
$$

If we suppose that $p_{18}$ is a free point, then $\Delta_{\nu}\left(E_{0}\right)$ is the triangle with coordinates

$$
\mathbf{0}=(0,0),(16,0) \text { and }\left(16, \frac{1}{16}\right) .
$$

Now consider another configuration of infinitely near points $\mathcal{C}_{\nu_{n}}$ for which the strict transforms of the projective line $L$ pass through $p_{1}, p_{2}$ and $p_{3}$. Then, $\nu_{n}$ is negative at infinity and non-minimal, since

$$
\hat{\mu}\left(\nu_{n}\right)=\nu_{n}\left(\varphi_{L}\right)=20 \text { and } \hat{\mu}\left(\nu_{n}\right)^{2}=400>256=\bar{\beta}_{g+1}\left(\nu_{n}\right) .
$$


Considering the exceptional curve valuation corresponding to a flag

$$
\left\{Z_{17} \supset E_{17} \supset\left\{p_{18}\right\}\right\}
$$

corresponding to this new configuration $\mathcal{C}_{\nu_{n}}$, it holds that the Newton-Okounkov body $\Delta_{\nu}\left(E_{0}\right)$ is determined by the vertices of coordinates

$$
\mathbf{0}=(0,0),\left(\frac{256}{20}, \frac{255}{20}\right) \text { and }(20,20)
$$

when $p_{18} \in E_{16} \cap E_{17}$, since $16=\eta \preccurlyeq r=17$. Otherwise, these vertices are

$$
\mathbf{0}=(0,0),\left(\frac{256}{20}, \frac{1}{20}\right) \text { and }(20,0)
$$

In what follows, we assume that $\nu$ is an exceptional curve valuation of $\mathbb{F}_{\delta}, \delta \geq 0$, which is non-minimal with respect to a big divisor $D \sim a F+b M$ on $\mathbb{F}_{\delta}$. Its first component will be the divisorial valuation $\nu_{r}$ of $\mathbb{F}_{\delta}$.

The divisor $D$ is also nef when $\delta=0$. Otherwise $(\delta \neq 0), D$ can be big and not nef. In this last case, the Zariski decomposition of the total transform $D^{*}$ on $Z=Z_{r}$ of $D$ is

$$
P_{D^{*}} \sim\left(b+\frac{a}{\delta}\right) M^{*} \text { and } N_{D^{*}}=\frac{-a}{\delta} \tilde{M}_{0}+\sum_{i=0}^{i_{M_{0}}} \frac{-a \nu_{i}\left(\varphi_{M_{0}}\right)}{\delta} E_{i},
$$

where $P_{D^{*}}$ (respectively, $N_{D^{*}}$ ) is the positive (respectively, negative) part of $D^{*}$ and $i_{M_{0}}$ indicates the last point in $C_{\nu_{r}}$ through which the strict transform of $M_{0}$ passes. In the following subsections, in virtue of Theorem 1.5.2 and [81, Lemma 1.10], we will distinguish two situations to compute $\Delta_{\nu}(D)$.

The first one corresponds to the case when the point $p_{r+1}$ is not in the support of the divisor $N_{D^{*}}$, denoted by $\operatorname{supp}\left(N_{D^{*}}\right)$. Here, we can also assume that the divisor $D$ is nef. Indeed, when $D \sim a F+b M$ is big and not nef, then $b>0$ and $-b \delta<a<0$ and, by [81, Lemma 1.10], the Newton-Okounkov body $\Delta_{\nu}(D)$ satisfies

$$
\Delta_{\nu}(D)=\Delta_{\nu}\left(P_{D}\right)=\left(b+\frac{a}{\delta}\right) \Delta_{\nu}(M) .
$$

Otherwise, the point $p_{r+1}$ belongs to $\operatorname{supp}\left(N_{D^{*}}\right)$. This fact happens if and only if $g^{*}=0, p_{1}$ is a special point, all the points in $\left\{p_{i}\right\}_{i=1}^{r+1}$ are free, $D$ is big but not nef, $i_{M_{0}}=r$ and $p_{r+1} \in \operatorname{supp}\left(\tilde{M}_{0}\right)$. Notice that we are in the first situation whenever $\delta=0$.

We conclude by noting that, when $p_{r+1} \notin \operatorname{supp}\left(N_{D^{*}}\right)$, one can write (3.2) as

$$
\hat{\mu}_{D}\left(\nu_{r}\right) \geq \sqrt{D^{2} \bar{\beta}_{g+1}\left(\nu_{r}\right)}
$$

since $\operatorname{vol}(D)=D^{2}$. Otherwise, we will replace $D$ by $P_{D}$.

In the forthcoming subsections we will explicitly describe the Newton-Okounkov body of a divisor $D$ as above with respect to an NPI exceptional curve valuation $\nu$ of $\mathbb{F}_{\delta}$. We begin with special valuations, where the first situation explained before could happen. 


\subsubsection{Newton-Okounkov bodies with respect to non-positive at in- finity special valuations}

Along this subsection we consider a big divisor $D \sim a F+b M$ on $\mathbb{F}_{\delta}, \delta \geq 0$, and an NPI special exceptional curve valuation $\nu$ of $\mathbb{F}_{\delta}$ which is non-minimal with respect to $D$. Also, we assume that $\nu_{r}$ is the first component of $\nu$.

Set $Z=Z_{r}$ the rational surface that $\nu_{r}$ defines and $E_{i}, 1 \leq i \leq r$, the exceptional divisors obtained in the sequence of point blowups defined by $\nu_{r}$. We denote by $E_{i}^{*}$ (respectively, $D^{*}$ ) the total transform of the exceptional divisor $E_{i}$ (respectively, the total transform of the divisor $D$ ) on $Z$.

For simplicity, the symbol $\theta_{1}^{r}(D)$ stands for the expression $a \nu_{r}\left(\varphi_{F_{1}}\right)-b \nu_{r}\left(\varphi_{M_{0}}\right)$, where $F_{1}$ is the fiber which contains $p$ and $M_{0}$ the special section; both on $\mathbb{F}_{\delta}$. If $\theta_{1}^{r}(D)$ vanishes, then either $a=b \nu_{r}\left(\varphi_{M_{0}}\right) / \nu_{r}\left(\varphi_{F_{1}}\right)$; or $\nu_{r}\left(\varphi_{M_{0}}\right)=0$ and $a=0$. Note that, under the second condition, some of the expressions we are going to introduce are not defined and they will not used when $\theta_{1}^{r}(D)=0$. In addition, when $p_{r+1} \in \operatorname{supp}\left(N_{D^{*}}\right), \theta_{1}^{r}(D)$ is negative.

Firstly, we state (and prove) three lemmas which will help us to obtain the Zariski decomposition of some key divisors for our goal.

Lemma 3.3.12. Let $D$ be a big and nef divisor on $\mathbb{F}_{\delta}$ and $\nu_{r}$ an NPI special divisorial valuation of $\mathbb{F}_{\delta}$. Set $\theta_{1}^{r}(D)$ the value above defined. Then the divisor on $Z$

$$
D_{1}=D^{*}-\frac{b}{\nu_{r}\left(\varphi_{F_{1}}\right)} \sum_{i=1}^{r} \nu_{r}\left(\mathfrak{m}_{i}\right) E_{i}^{*}\left(\text { respectively, } D_{2}=D^{*}-\frac{a}{\nu_{r}\left(\varphi_{M_{0}}\right)} \sum_{i=1}^{r} \nu_{r}\left(\mathfrak{m}_{i}\right) E_{i}^{*}\right)
$$

is nef if $\theta_{1}^{r}(D) \geq 0$ (respectively, $\theta_{1}^{r}(D)<0$ ).

Proof. We only show that $D_{1}$ is nef. The proof for $D_{2}$ runs similarly. Taking into account that $b$ is positive, it holds that

$$
\begin{aligned}
D_{1} & =D^{*}-\frac{b}{\nu_{r}\left(\varphi_{F_{1}}\right)} \sum_{i=1}^{r} \nu_{r}\left(\mathfrak{m}_{i}\right) E_{i}^{*} \\
& \sim \frac{b}{\nu_{r}\left(\varphi_{F_{1}}\right)}\left(\frac{a \nu_{r}\left(\varphi_{F_{1}}\right)}{b} F^{*}+\nu_{r}\left(\varphi_{F_{1}}\right) M^{*}-\sum_{i=1}^{r} \nu_{r}\left(\mathfrak{m}_{i}\right) E_{i}^{*}\right) \\
& =\frac{b}{\nu_{r}\left(\varphi_{F_{1}}\right)}\left(\frac{\theta_{1}^{r}(D)}{b} F^{*}+\Lambda_{r}\right),
\end{aligned}
$$

where $\Lambda_{r}=\nu_{r}\left(\varphi_{M_{0}}\right) F^{*}+\nu_{r}\left(\varphi_{F_{1}}\right) M^{*}-\sum_{i=1}^{r} \nu_{r}\left(\mathfrak{m}_{i}\right) E_{i}^{*}$. As $\theta_{1}^{r}(D)$ is non-negative and $F^{*}$ and $\Lambda_{r}$ are nef divisors, by Theorem 2.3.7, $D_{1}$ is also nef.

Lemma 3.3.13. Let $\nu_{r}$ be an NPI special divisorial valuation of $\mathbb{F}_{\delta}$ and $Z$ the surface defined by $\nu_{r}$. Set $D \sim a F+b M$ a big divisor on $\mathbb{F}_{\delta}$ and, as above, denote by $\theta_{1}^{r}(D)$ the expression $a \nu_{r}\left(\varphi_{F_{1}}\right)-b \nu_{r}\left(\varphi_{M_{0}}\right)$. Let $t_{1}, t_{2}, t_{3}$ and $t_{4}$ be the following four rational numbers:

$$
t_{1}=\frac{b}{\nu_{r}\left(\varphi_{F_{1}}\right)} \bar{\beta}_{g+1}\left(\nu_{r}\right), \quad t_{2}=\frac{b}{\nu_{r}\left(\varphi_{F_{1}}\right)} \bar{\beta}_{g+1}\left(\nu_{r}\right)+\theta_{1}^{r}(D)
$$




$$
t_{3}=\frac{a}{\nu_{r}\left(\varphi_{M_{0}}\right)} \bar{\beta}_{g+1}\left(\nu_{r}\right) \text { and } t_{4}=\frac{(a+b \delta) \bar{\beta}_{g+1}\left(\nu_{r}\right)-\theta_{1}^{r}(D) \nu_{r}\left(\varphi_{M_{0}}\right)}{\nu_{r}\left(\varphi_{M_{0}}\right)+\delta \nu_{r}\left(\varphi_{F_{1}}\right)} .
$$

(1) Assume that the divisor $D$ is nef. The values $t_{1}$ and $t_{2}$ (respectively, $t_{3}$ and $t_{4}$ ) belong to the set

$$
T_{D, \nu_{r}}:=\left\{t \in \mathbb{Q} \mid 0 \leq t \leq \hat{\mu}_{D}\left(\nu_{r}\right)\right\}
$$

if $\theta_{1}^{r}(D) \geq 0$ (respectively, $\left.\theta_{1}^{r}(D)<0\right)$.

(2) Assume that $p_{r+1} \in \operatorname{supp}\left(N_{D^{*}}\right)$. Then, the value $t_{4}$ satisfies

$$
0<-a \nu_{r}\left(\varphi_{M_{0}}\right) / \delta<t_{4} \leq \hat{\mu}_{D}\left(\nu_{r}\right)
$$

Proof. We only prove that $t_{1}, t_{2} \leq \hat{\mu}_{D}\left(\nu_{r}\right)$ for the first part. A proof for the values $t_{3}$ and $t_{4}$ in our second assertion follows from an analogous reasoning.

We start by proving that $t_{1} \leq \hat{\mu}_{D}\left(\nu_{r}\right)$ when $\theta_{1}^{r}(D)$ is non-negative. Consider the nef divisor on $Z, D_{1}=D^{*}-\frac{b}{\nu_{r}\left(\varphi_{F_{1}}\right)} \sum_{i=1}^{r} \nu_{r}\left(\mathfrak{m}_{i}\right) E_{i}^{*}$, defined in Lemma 3.3.12. For any curve $C \in|m D|, m \in \mathbb{Z}_{>0}$, it holds that

$$
m\left(2 a b+b^{2} \delta\right)-\frac{b}{\nu_{r}\left(\varphi_{F_{1}}\right)} \nu_{r}\left(\varphi_{C}\right)=D_{1} \cdot \tilde{C} \geq 0
$$

where $\tilde{C}$ is the strict transform of $C$ under the birational map that $\nu_{r}$ defines. Consequently, one has

$$
2 a b+b^{2} \delta \geq \frac{b}{\nu_{r}\left(\varphi_{F_{1}}\right)} \hat{\mu}_{D}\left(\nu_{r}\right)
$$

and together with (3.7) we obtain

$$
\hat{\mu}_{D}\left(\nu_{r}\right) \geq \frac{D^{2} \bar{\beta}_{g+1}\left(\nu_{r}\right)}{\hat{\mu}_{D}\left(\nu_{r}\right)}=\frac{\left(2 a b+b^{2} \delta\right) \bar{\beta}_{g+1}\left(\nu_{r}\right)}{\hat{\mu}_{D}\left(\nu_{r}\right)} \geq \frac{b \bar{\beta}_{g+1}\left(\nu_{r}\right)}{\nu_{r}\left(\varphi_{F_{1}}\right)},
$$

which show our claim.

Now we are going to prove that $t_{2} \leq \hat{\mu}_{D}\left(\nu_{r}\right)$ if $\theta_{1}^{r}(D) \geq 0$. Using Theorem 3.2.1, it is sufficient to see

$$
b \bar{\beta}_{g+1}\left(\nu_{r}\right) \leq b\left(2 \nu_{r}\left(\varphi_{M_{0}}\right) \nu_{r}\left(\varphi_{F_{1}}\right)+\delta \nu_{r}\left(\varphi_{F_{1}}\right)^{2}\right)
$$

which is true by Theorem 2.3.7 and the fact that $b$ is positive.

To conclude, we show (2), that is, $0<-a \nu_{r}\left(\varphi_{M_{0}}\right) / \delta<t_{4}$ when $D \sim a F+b M$ is big and $p_{r+1} \in \operatorname{supp}\left(N_{D^{*}}\right)$. It is easy to check that

$$
t_{4}+\frac{a \nu_{r}\left(\varphi_{M_{0}}\right)}{\delta}=\frac{(a+b \delta)\left(\nu_{r}\left(\varphi_{M_{0}}\right)^{2}+\delta \bar{\beta}_{g+1}\left(\nu_{r}\right)\right)}{\delta\left(\nu_{r}\left(\varphi_{M_{0}}\right)+\delta \nu_{r}\left(\varphi_{F_{1}}\right)\right)}>0,
$$

where the inequality holds since, as mentioned at the end of the last subsection, $D$ is big but not nef and then $-b \delta<a<0$, which completes the proof.

A consequence of the above lemma and Theorem 3.2.1 is the following result. 
Corollary 3.3.14. Let $D$ be a big divisor on $\mathbb{F}_{\delta}, \delta \geq 0$, and $\nu_{r}$ an NPI special divisorial valuation of $\mathbb{F}_{\delta}$. Consider the values $\theta_{1}^{r}(D), t_{2}$ and $t_{4}$ given in Lemma 3.3.13. Then,

(a) Assume that $\theta_{1}^{r}(D) \geq 0$, then $2 \nu_{r}\left(\varphi_{M_{0}}\right) \nu_{r}\left(\varphi_{F_{1}}\right)+\delta \nu_{r}\left(\varphi_{F_{1}}\right)^{2}=\bar{\beta}_{g+1}\left(\nu_{r}\right)$ if and only if $t_{2}=\hat{\mu}_{D}\left(\nu_{r}\right)$.

(b) Otherwise $\left(\theta_{1}^{r}(D)<0\right)$, the equality $2 \nu_{r}\left(\varphi_{M_{0}}\right) \nu_{r}\left(\varphi_{F_{1}}\right)+\delta \nu_{r}\left(\varphi_{F_{1}}\right)^{2}=\bar{\beta}_{g+1}\left(\nu_{r}\right)$ holds if and only if $t_{4}=\hat{\mu}_{D}\left(\nu_{r}\right)$.

Proof. We only show a proof for Item (a). A similar argument shows Item (b). We start assuming that $2 \nu_{r}\left(\varphi_{M_{0}}\right) \nu_{r}\left(\varphi_{F_{1}}\right)+\delta \nu_{r}\left(\varphi_{F_{1}}\right)^{2}=\bar{\beta}_{g+1}\left(\nu_{r}\right)$. Applying the above equality to the expression of $t_{2}$ in Lemma 3.3.13, the next equalities follow:

$$
\begin{aligned}
t_{2} & =2 b \nu_{r}\left(\varphi_{M_{0}}\right)+\delta b \nu_{r}\left(\varphi_{F_{1}}\right)+a \nu_{r}\left(\varphi_{F_{1}}\right)-b \nu_{r}\left(\varphi_{M_{0}}\right) \\
& =(a+b \delta) \nu_{r}\left(\varphi_{F_{1}}\right)+b \nu_{r}\left(\varphi_{M_{0}}\right)=\hat{\mu}_{D}\left(\nu_{r}\right),
\end{aligned}
$$

which show one implication. For the other one, assume that $t_{2}=\hat{\mu}_{D}\left(\nu_{r}\right)$. Then,

$$
0=\hat{\mu}_{D}\left(\nu_{r}\right)-t_{2}=b \delta \nu_{r}\left(\varphi_{F_{1}}\right)+2 b \nu_{r}\left(\varphi_{M_{0}}\right)-\frac{b}{\nu_{r}\left(\varphi_{F_{1}}\right)} \bar{\beta}_{g+1}\left(\nu_{r}\right),
$$

and, as $b$ and $\nu_{r}\left(\varphi_{F_{1}}\right)$ are positive, the result follows by multiplying $\nu_{r}\left(\varphi_{F_{1}}\right) / b$.

Remark 3.3.15. Some extra information can be given on the values $t_{i}, 1 \leq i \leq 4$.

(a) The valuation $\nu_{r}$ is minimal with respect to a big and nef divisor $D$ on $\mathbb{F}_{\delta}$ if and only if

$\hat{\mu}_{D}\left(\nu_{r}\right)=\frac{b \bar{\beta}_{g+1}\left(\nu_{r}\right)}{\nu_{r}\left(\varphi_{F_{1}}\right)}=t_{1}=t_{2}\left(=\frac{a \bar{\beta}_{g+1}\left(\nu_{r}\right)}{\nu_{r}\left(\varphi_{M_{0}}\right)}=t_{3}=t_{4}\right.$, when $\left.\nu_{r}\left(\varphi_{M_{0}}\right) \neq 0\right)$.

In fact, the equalities $t_{1}=t_{2}$ and $\hat{\mu}_{D}\left(\nu_{r}\right)=t_{2}$ are equivalent to those given in Corollary 3.2.3 by Corollary 3.3.14.

(b) Assume that $\nu_{r}$ is non-minimal with respect to a big and nef divisor $D$ on $\mathbb{F}_{\delta}$. Then,

(b.1) The value $\theta_{1}^{r}(D)$ vanishes if and only if $\hat{\mu}_{D}\left(\nu_{r}\right)>t_{1}=t_{2}\left(=t_{3}=t_{4}\right.$, when $\left.\nu_{r}\left(\varphi_{M_{0}}\right) \neq 0\right)$.

(b.2) Consider that $\theta_{1}^{r}(D)>0$. The inequality

$$
2 \nu_{r}\left(\varphi_{M_{0}}\right) \nu_{r}\left(\varphi_{F_{1}}\right)+\delta \nu_{r}\left(\varphi_{F_{1}}\right)^{2} \geq \bar{\beta}_{g+1}\left(\nu_{r}\right)
$$

holds if and only if $\hat{\mu}_{D}\left(\nu_{r}\right) \geq t_{2}>t_{1}>0$.

(b.3) Otherwise $\left(\theta_{1}^{r}(D)<0\right)$. The conditions

$$
2 \nu_{r}\left(\varphi_{M_{0}}\right) \nu_{r}\left(\varphi_{F_{1}}\right)+\delta \nu_{r}\left(\varphi_{F_{1}}\right)^{2} \geq \bar{\beta}_{g+1}\left(\nu_{r}\right) \text { and } a \geq 0
$$

hold if and only if $\hat{\mu}_{D}\left(\nu_{r}\right) \geq t_{4}>t_{3} \geq 0$. 
Lemma 3.3.16. Keep the notation of Lemma 3.3.13. Assume that $\nu_{r}$ is non-minimal with respect to a big divisor $D \sim a F+b M$ on $\mathbb{F}_{\delta}$.

(1) Suppose that $D$ is also a nef divisor. Then, the intersection matrices defined by the sets $\left\{\tilde{F}_{1}, E_{1}, E_{2}, \ldots, E_{r-1}\right\}$ and $\left\{\tilde{M}_{0}, E_{1}, E_{2}, \ldots, E_{r-1}\right\}$ are negative definite.

(2) Suppose that $p_{r+1} \in \operatorname{supp}\left(N_{D^{*}}\right)$, it holds that the set $\left\{\tilde{M}_{0}, E_{1}, E_{2}, \ldots, E_{r-1}\right\}$ determines a negative definite intersection matrix.

Proof. Set $D_{1}$ the divisor given in Lemma 3.3.12. There we proved that $D_{1}$ is nef. Now we are going to show that it is also a big divisor. Indeed, as $\nu_{r}$ is non-minimal with respect to $D$ and taking into account (3.8), one gets the following inequalities

$$
\frac{D^{2} \bar{\beta}_{g+1}\left(\nu_{r}\right)}{\hat{\mu}_{D}\left(\nu_{r}\right)} \geq \frac{b \bar{\beta}_{g+1}\left(\nu_{r}\right)}{\nu_{r}\left(\varphi_{F_{1}}\right)} \text { and } 1>\frac{D^{2} \bar{\beta}_{g+1}\left(\nu_{r}\right)}{\hat{\mu}_{D}\left(\nu_{r}\right)^{2}}
$$

which allow us to show that $D_{1}^{2}>0$ since

$$
D_{1}^{2}=D^{2}-\frac{b^{2} \bar{\beta}_{g+1}\left(\nu_{r}\right)}{\nu_{r}\left(\varphi_{F_{1}}\right)^{2}} \geq D^{2}-\frac{b \hat{\mu}_{D}\left(\nu_{r}\right)}{\nu_{r}\left(\varphi_{F_{1}}\right)}\left(\frac{D^{2} \bar{\beta}_{g+1}\left(\nu_{r}\right)}{\hat{\mu}_{D}\left(\nu_{r}\right)^{2}}\right)>D^{2}-\frac{b \hat{\mu}_{D}\left(\nu_{r}\right)}{\nu_{r}\left(\varphi_{F_{1}}\right)} \geq 0 .
$$

As a consequence, using Theorem 1.4.7, it holds that $D_{1}$ is big. In addition, $D_{1}$ is orthogonal to $\tilde{F}_{1}$ and to $E_{i}$, for $1 \leq i \leq r-1$, and consequently the $\operatorname{set}\left\{\tilde{F}_{1}, E_{1}, E_{2}, \ldots\right.$, $\left.E_{r-1}\right\}$ generates a negative definite intersection matrix by Proposition 1.4.12.

The remaining cases follow from a similar argument using, either the divisor $D_{2}$ defined in Lemma 3.3.12 or the big and nef divisor $(b+a / \delta) M^{*}$.

The following proposition provides the positive and negative parts of the Zariski decomposition of certain divisors on $Z$ which we will use to describe the NewtonOkounkov body of a big divisor on $\mathbb{F}_{\delta}, \delta \geq 0$.

Proposition 3.3.17. Let $\nu_{r}$ be an NPI special divisorial valuation of $\mathbb{F}_{\delta}$ and $Z=Z_{r}$ the rational surface that $\nu_{r}$ defines. Set $\nu_{i}$ the NPI special divisorial valuation which the divisor $E_{i}$ defines, for $i \in\{1,2, \ldots, r-1\}$. Consider a big divisor $D \sim a F+b M$ on $\mathbb{F}_{\delta}$ and assume that $\nu_{r}$ is non-minimal with respect to $D$. Set $\theta_{1}^{r}(D)=a \nu_{r}\left(\varphi_{F_{1}}\right)-$ $b \nu_{r}\left(\varphi_{M_{0}}\right)$ and the divisor on $Z, \Lambda_{r}=\nu_{r}\left(\varphi_{M_{0}}\right) F^{*}+\nu_{r}\left(\varphi_{F_{1}}\right) M^{*}-\sum_{i=1}^{r} \nu_{r}\left(\mathfrak{m}_{i}\right) E_{i}^{*}$. Let $D_{1}$ and $D_{2}$ be the divisors given in Lemma 3.3.12 and $t_{1}, t_{2}, t_{3}$ and $t_{4}$ the rational numbers defined in Lemma 3.3.13.

(1) Assume that $D$ is also a nef divisor.

(a) Suppose that $\theta_{1}^{r}(D) \geq 0$. The positive and negative parts of the Zariski decomposition of the divisors on $Z D_{t_{1}}:=D^{*}-t_{1} E_{r}$, and $D_{t_{2}}:=D^{*}-t_{2} E_{r}$ 
are

$$
\begin{gathered}
P_{D_{t_{1}}} \sim D_{1} \text { and } N_{D_{t_{1}}}=\frac{b}{\nu_{r}\left(\varphi_{F_{1}}\right)} \sum_{i=1}^{r-1} \nu_{r}\left(\varphi_{i}\right) E_{i}, \\
\text { and } P_{D_{t_{2}}} \sim \frac{b}{\nu_{r}\left(\varphi_{F_{1}}\right)} \Lambda_{r} \text { and } \\
N_{D_{t_{2}}}=\frac{\theta_{1}^{r}(D)}{\nu_{r}\left(\varphi_{F_{1}}\right)} \tilde{F}_{1}+\sum_{i=1}^{r-1} \frac{b \nu_{r}\left(\varphi_{i}\right)+\theta_{1}^{r}(D) \nu_{i}\left(\varphi_{F_{1}}\right)}{\nu_{r}\left(\varphi_{F_{1}}\right)} E_{i} .
\end{gathered}
$$

(b) Otherwise, the positive and negative parts of the Zariski decomposition of the divisors $D_{t_{3}}:=D^{*}-t_{3} E_{r}$, and $D_{t_{4}}:=D^{*}-t_{4} E_{r}$ are

$$
\begin{gathered}
P_{D_{t_{3}}} \sim D_{2} \text { and } N_{D_{t_{3}}}=\frac{a}{\nu_{r}\left(\varphi_{M_{0}}\right)} \sum_{i=1}^{r-1} \nu_{r}\left(\varphi_{i}\right) E_{i}, \\
\text { and } P_{D_{t_{4}}} \sim \frac{a+b \delta}{\nu_{r}\left(\varphi_{M_{0}}\right)+\delta \nu_{r}\left(\varphi_{F_{1}}\right)} \Lambda_{r} \text { and } \\
N_{D_{t_{4}}}=\left(\frac{-\theta_{1}^{r}(D)}{\nu_{r}\left(\varphi_{M_{0}}\right)+\delta \nu_{r}\left(\varphi_{F_{1}}\right)}\right) \tilde{M}_{0} \\
+\sum_{i=1}^{r-1} \frac{(a+b \delta) \nu_{r}\left(\varphi_{i}\right)-\theta_{1}^{r}(D) \nu_{i}\left(\varphi_{M_{0}}\right)}{\nu_{r}\left(\varphi_{M_{0}}\right)+\delta \nu_{r}\left(\varphi_{F_{1}}\right)} E_{i} .
\end{gathered}
$$

(2) Assume that $p_{r+1} \in \operatorname{supp}\left(N_{D^{*}}\right)$. Then the previous divisors $P_{D_{t_{4}}}$ and $N_{D_{t_{4}}}$ are the positive and negative parts of $D_{t_{4}}$.

Proof. We only show a proof for (a); a proof for the remaining cases follows from a similar argument. We begin with the Zariski decomposition of $D_{t_{1}}$. It is immediate that $P_{D_{t_{1}}}+N_{D_{t_{1}}} \sim D_{t_{1}}$. In addition, the divisor $P_{D_{t_{1}}}$ is nef by Lemma 3.3.12. Finally, each component of $N_{D_{t_{1}}}$ is orthogonal to $P_{D_{t_{1}}}$ by the proximity equalities, and they determine an intersection matrix which is negative definite.

To conclude, we will prove the result for $D_{t_{2}}$. The divisor $P_{D_{t_{2}}}$ is nef and orthogonal to each component of $N_{D_{t_{2}}}$ by Proposition 2.3.1 and Theorem 2.3.7. Moreover, it is clear that the intersection matrix given by the components of $N_{D_{t_{2}}}$ is negative definite by Lemma 3.3.16. Finally, the fact that $D_{t_{2}} \sim P_{D_{t_{2}}}+N_{D_{t_{2}}}$ follows from summing the next two expressions

$$
D-\frac{b}{\nu_{r}\left(\varphi_{F_{1}}\right)} \bar{\beta}_{g+1}\left(\nu_{r}\right) E_{r} \sim \frac{b}{\nu_{r}\left(\varphi_{F_{1}}\right)} \Lambda_{r}+\frac{\theta_{1}^{r}(D)}{\nu_{r}\left(\varphi_{F_{1}}\right)} F^{*}+\frac{b}{\nu_{r}\left(\varphi_{F_{1}}\right)} \sum_{i=1}^{r-1} \nu_{r}\left(\varphi_{i}\right) E_{i}
$$

and

$$
-\theta_{1}^{r}(D) E_{r}=\frac{\theta_{1}^{r}(D)}{\nu_{r}\left(\varphi_{F_{1}}\right)}\left(\sum_{i=1}^{r-1} \nu_{i}\left(\varphi_{F_{1}}\right) E_{i}-\sum_{i=1}^{i_{F_{1}}} E_{i}^{*}\right),
$$

and from considering that $\tilde{F}_{1} \sim F^{*}-\sum_{i=1}^{i_{F_{1}}} E_{i}^{*}$, where $i_{F_{1}}$ indicates the last point in the configuration of infinitely near points $\mathcal{C}_{\nu_{r}}$ of the valuation $\nu_{r}$ through which the strict transform of $F_{1}$ goes, which proves the result. 
Remark 3.3.18. Keep the notation used at the end of Subsection 1.5.2, in Lemmas 3.3.12, 3.3.13 and 3.3.16 and in Proposition 3.3.17. The ray $\left[D_{t}\right]:=\left[D^{*}\right]-t\left[E_{r}\right]$, where $0 \leq t \leq \hat{\mu}_{D}\left(\nu_{r}\right)$, crosses the interior of $\mathrm{NE}(Z)$ heading towards the face of $\mathrm{NE}(Z)$ spanned by the classes of the divisors $\tilde{F}_{1}, \tilde{M}_{0}, E_{1}, E_{2}, \ldots, E_{r-1}$. Indeed, notice that, when $t$ is a small enough value, it holds that

$$
\operatorname{Null}\left(D_{t}\right)=\left\{E_{1}, E_{2}, \ldots, E_{r-1}\right\}=\operatorname{Neg}\left(D_{t}\right) .
$$

When $\theta_{1}^{r}(D)>0$, the ray $\left[D^{*}\right]-t\left[E_{r}\right]$ is contained in the boundary of a Zariski chamber for the values $t_{1}$ and $t_{2}$ since

$$
\operatorname{Neg}\left(D_{t_{1}}\right)=\left\{E_{1}, E_{2}, \ldots, E_{r-1}\right\} \text { and } \operatorname{Null}\left(P_{D_{t_{1}}}\right)=\left\{\tilde{F}_{1}, E_{1}, E_{2}, \ldots, E_{r-1}\right\}
$$

and

$$
\operatorname{Neg}\left(D_{t_{2}}\right)=\left\{\tilde{F}_{1}, E_{1}, E_{2}, \ldots, E_{r-1}\right\} \text { and } \operatorname{Null}\left(P_{D_{t_{2}}}\right)=\left\{\tilde{F}_{1}, \tilde{M}_{0}, E_{1}, E_{2}, \ldots, E_{r-1}\right\} .
$$

An analogous result happens if $\theta_{1}^{r}(D)<0$. In this case the ray $\left[D^{*}\right]-t\left[E_{r}\right]$ is in the boundary of a Zariski chamber for the values $t_{3}$ and $t_{4}$ because

$$
\operatorname{Neg}\left(D_{t_{3}}\right)=\left\{E_{1}, E_{2}, \ldots, E_{r-1}\right\} \text { and } \operatorname{Null}\left(P_{D_{t_{3}}}\right)=\left\{\tilde{M}_{0}, E_{1}, E_{2}, \ldots, E_{r-1}\right\},
$$

and

$$
\operatorname{Neg}\left(D_{t_{4}}\right)=\left\{\tilde{M}_{0}, E_{1}, E_{2}, \ldots, E_{r-1}\right\} \text { and } \operatorname{Null}\left(P_{D_{t_{4}}}\right)=\left\{\tilde{F}_{1}, \tilde{M}_{0}, E_{1}, E_{2}, \ldots, E_{r-1}\right\}
$$

Finally, for $\theta_{1}^{r}(D)=0$, the ray is in the boundary of several Zariski chambers for the value $t_{1}=t_{2}$, since

$$
\operatorname{Neg}\left(D_{t_{1}}\right)=\left\{E_{1}, E_{2}, \ldots, E_{r-1}\right\} \text { and } \operatorname{Null}\left(P_{D_{t_{1}}}\right)=\left\{\tilde{F}_{1}, \tilde{M}_{0}, E_{1}, E_{2}, \ldots, E_{r-1}\right\} .
$$

Figure 3.2 depicts the above situations, where each case has a different color; the vertical line, denoted $\left[\Lambda_{n}\right]^{\perp}$, represents the face of $\mathrm{NE}(Z)$ spanned by the classes of the divisors $\tilde{F}_{1}, \tilde{M}_{0}, E_{1}, E_{2}, \ldots, E_{r-1}$; and the Zariski chambers $\Sigma_{1}, \Sigma_{2}, \Sigma_{3}$ and $\Sigma_{4}$ below defined are delimited by discontinuous lines.

$$
\begin{gathered}
\Sigma_{1}:=\left\{[\xi] \in \operatorname{Big}(Z) \mid \operatorname{Neg}(\xi)=\operatorname{Null}(\xi)=\left\{\left[E_{1}\right],\left[E_{2}\right], \ldots,\left[E_{r-1}\right]\right\}\right\}, \\
\Sigma_{2}:=\left\{[\xi] \in \operatorname{Big}(Z) \mid \operatorname{Neg}(\xi)=\operatorname{Null}(\xi)=\left\{\left[\tilde{F}_{1}\right],\left[E_{1}\right],\left[E_{2}\right], \ldots,\left[E_{r-1}\right]\right\}\right\}, \\
\Sigma_{3}:=\left\{[\xi] \in \operatorname{Big}(Z) \mid \operatorname{Neg}(\xi)=\operatorname{Null}(\xi)=\left\{\left[\tilde{M}_{0}\right],\left[E_{1}\right],\left[E_{2}\right], \ldots,\left[E_{r-1}\right]\right\}\right\} \text { and } \\
\Sigma_{4}:=\left\{[\xi] \in \operatorname{Big}(Z) \mid \operatorname{Neg}(\xi)=\operatorname{Null}(\xi)=\left\{\left[\tilde{F}_{1}\right],\left[\tilde{M}_{0}\right],\left[E_{1}\right],\left[E_{2}\right], \ldots,\left[E_{r-1}\right]\right\}\right\} .
\end{gathered}
$$

Now we are going to state the three main results in this subsection. Recall that $D \sim a F+b M$ is a big divisor on $\mathbb{F}_{\delta}$ and $\nu$ an NPI special exceptional curve valuation which is non-minimal with respect to $D$ and whose first component is $\nu_{r}$. 


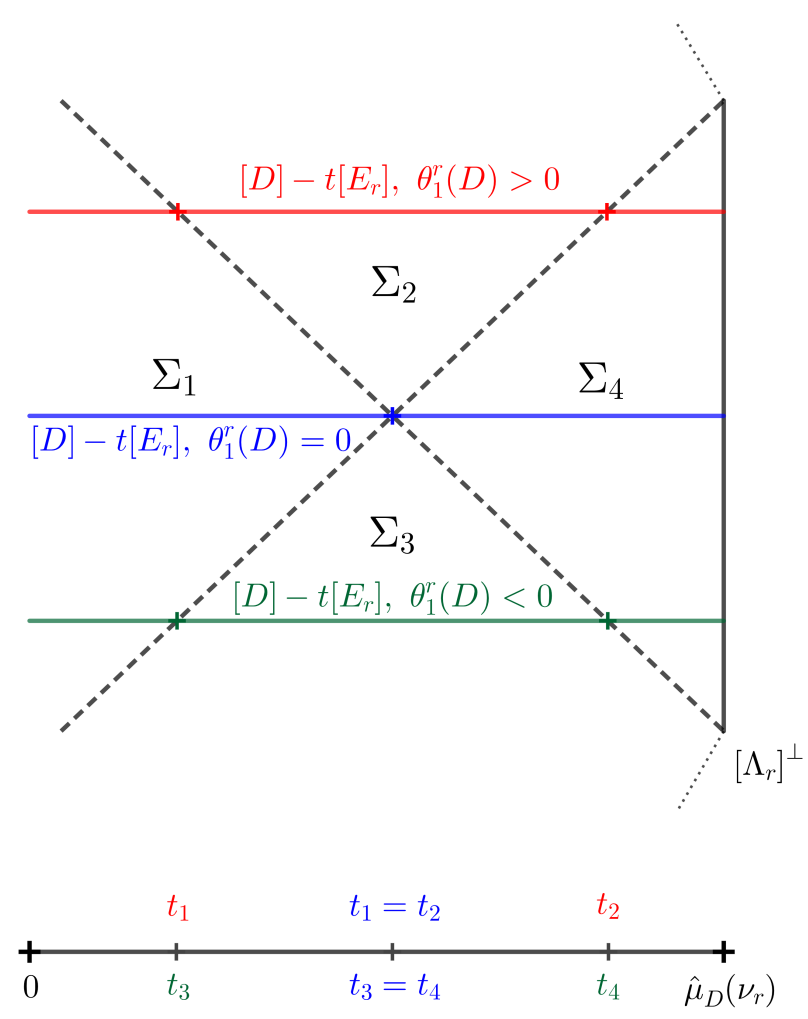

Figure 3.2: Local description of the cone of curves $\mathrm{NE}(Z)$ of a rational surface $Z$ given by a non-positive at infinity special divisorial valuation of $\mathbb{F}_{\delta}$.

The results explicitly describe the Newton-Okounkov bodies $\Delta_{\nu}(D)$. We distinguish three cases:

Case A: Either $g^{*}>0$, or $g^{*}=0, \nu\left(\varphi_{F_{1}}\right) \neq \bar{\beta}_{1}(\nu)$ and $\nu\left(\varphi_{M_{0}}\right) \neq \bar{\beta}_{1}(\nu)$.

Case B: The integer $g^{*}$ equals 0 and $\nu\left(\varphi_{F_{1}}\right)=\bar{\beta}_{1}(\nu)$.

Case C: The integer $g^{*}$ equals 0 and $\nu\left(\varphi_{M_{0}}\right)=\bar{\beta}_{1}(\nu)$.

Before starting our description for Case A, we give a useful property.

Lemma 3.3.19. Let $\nu$ be an NPI special exceptional curve valuation of $\mathbb{F}_{\delta}$ and $\mathcal{C}_{\nu}=\left\{p_{i}\right\}_{i \geq 1}$ its configuration of infinitely near points such that $p_{i} \rightarrow p_{r}$ for all $i>r$. Consider the cases described before.

(a) Suppose that $p_{r+1}$ is the satellite point $E_{\eta} \cap E_{r}, \eta \neq r$.

(a.1) Assume we are in Case A. Then,

$$
\nu_{\eta}\left(\varphi_{F_{1}}\right)=\nu_{r}\left(\varphi_{F_{1}}\right) \cdot \frac{\bar{\beta}_{0}\left(\nu_{\eta}\right)}{\bar{\beta}_{0}\left(\nu_{r}\right)} \text { and } \nu_{\eta}\left(\varphi_{M_{0}}\right)=\nu_{r}\left(\varphi_{M_{0}}\right) \cdot \frac{\bar{\beta}_{0}\left(\nu_{\eta}\right)}{\bar{\beta}_{0}\left(\nu_{r}\right)} .
$$

(a.2) Assume we are in Case B. Then,

$$
\nu_{\eta}\left(\varphi_{F_{1}}\right)=\nu_{r}\left(\varphi_{F_{1}}\right) \cdot \frac{\bar{\beta}_{g^{*}+1}\left(\nu_{\eta}\right)}{\bar{\beta}_{g^{*}+1}\left(\nu_{r}\right)} \text { and } \nu_{\eta}\left(\varphi_{M_{0}}\right)=\nu_{r}\left(\varphi_{M_{0}}\right) \cdot \frac{\bar{\beta}_{0}\left(\nu_{\eta}\right)}{\bar{\beta}_{0}\left(\nu_{r}\right)} \text {. }
$$


(a.3) Assume we are in Case C. Then,

$$
\nu_{\eta}\left(\varphi_{F_{1}}\right)=\nu_{r}\left(\varphi_{F_{1}}\right) \cdot \frac{\bar{\beta}_{0}\left(\nu_{\eta}\right)}{\bar{\beta}_{0}\left(\nu_{r}\right)} \text { and } \nu_{\eta}\left(\varphi_{M_{0}}\right)=\nu_{r}\left(\varphi_{M_{0}}\right) \cdot \frac{\bar{\beta}_{g^{*}+1}\left(\nu_{\eta}\right)}{\bar{\beta}_{g^{*}+1}\left(\nu_{r}\right)} \text {. }
$$

(b) Otherwise ( $p_{r+1}$ is a free point).

(b.1) Assume we are in Case A. Then,

$$
\nu\left(\varphi_{F_{1}}\right)=\left(\nu_{r}\left(\varphi_{F_{1}}\right), 0\right) \text { and } \nu\left(\varphi_{M_{0}}\right)=\left(\nu_{r}\left(\varphi_{M_{0}}\right), 0\right) .
$$

(b.2) Assume we are in Case B. Then,

$$
\nu\left(\varphi_{F_{1}}\right)=\left(\nu_{r}\left(\varphi_{F_{1}}\right), 1\right) \text { and } \nu\left(\varphi_{M_{0}}\right)=\left(\nu_{r}\left(\varphi_{M_{0}}\right), 0\right) .
$$

(b.3) Assume we are in Case C. Then,

$$
\nu\left(\varphi_{F_{1}}\right)=\left(\nu_{r}\left(\varphi_{F_{1}}\right), 0\right) \text { and } \nu\left(\varphi_{M_{0}}\right)=\left(\nu_{r}\left(\varphi_{M_{0}}\right), 1\right) .
$$

Proof. The result easily follows from the next observations. The value $\nu\left(\varphi_{F_{1}}\right)$ equals $\bar{\beta}_{1}(\nu)$ when the strict transform of the fiber $F_{1}$ goes through all initial free points of $\mathcal{C}_{\nu}$. Otherwise, $\nu\left(\varphi_{F_{1}}\right)=s_{F_{1}} \bar{\beta}_{0}(\nu)$ for a positive integer $s_{F_{1}}$. Similarly, the value $\nu\left(\varphi_{M_{0}}\right)$ is equals to either $\bar{\beta}_{1}(\nu)$, or $s_{M_{0}} \bar{\beta}_{0}(\nu)$, for a non-negative integer $s_{M_{0}}$. Finally at most one of the strict transforms of $F_{1}$ and $M_{0}$ pass through $p_{2}$.

We begin with Case A. Here $D$ can be also considered a nef divisor without loss of generality, since $\Delta_{\nu}(D)$ with $D$ big and not nef can be obtained as we said in the paragraphs before Subsection 3.3.1. Following Subsection 1.5.2, the NewtonOkounkov body $\Delta_{\nu}(D)$ can be seen as the set

$$
\left\{(t, y) \in \mathbb{R}^{2} \mid 0 \leq t \leq \hat{\mu}_{D}\left(\nu_{r}\right) \text { and } \alpha(t) \leq y \leq \beta(t)\right\},
$$

where $\alpha(t):=\operatorname{ord}_{p_{r+1}}\left(\left.N_{D_{t}}\right|_{E_{r}}\right)$ and $\beta(t):=\alpha(t)+P_{D_{t}} \cdot E_{r}$ for all $t \in\left[0, \hat{\mu}_{D}\left(\nu_{r}\right)\right]$; $P_{D_{t}}$ and $N_{D_{t}}$ being the positive part and the negative part of the divisor on $Z$ $D_{t}=D^{*}-t E_{r}$, respectively. As a result, using Proposition 3.3.17, some points which belong to $\Delta_{\nu}(D)$ are

$$
\begin{gathered}
Q_{1}=\left(\frac{b \bar{\beta}_{g+1}\left(\nu_{r}\right)}{\nu_{r}\left(\varphi_{F_{1}}\right)}, \frac{b \nu_{r}\left(\varphi_{\eta}\right)}{\nu_{r}\left(\varphi_{F_{1}}\right)}\right)\left(\text { respectively, } Q_{1}=\left(\frac{b \bar{\beta}_{g+1}\left(\nu_{r}\right)}{\nu_{r}\left(\varphi_{F_{1}}\right)}, 0\right)\right) \\
Q_{2}=Q_{1}+\left(0, \frac{b}{\nu_{r}\left(\varphi_{F_{1}}\right)}\right) \\
Q_{3}=\left(\frac{b \bar{\beta}_{g+1}\left(\nu_{r}\right)}{\nu_{r}\left(\varphi_{F_{1}}\right)}+\theta_{1}^{r}(D), \frac{b \nu_{r}\left(\varphi_{\eta}\right)+\theta_{1}^{r}(D) \nu_{\eta}\left(\varphi_{F_{1}}\right)}{\nu_{r}\left(\varphi_{F_{1}}\right)}\right) \\
\left(\text { respectively, } Q_{3}=\left(\frac{b \bar{\beta}_{g+1}\left(\nu_{r}\right)}{\nu_{r}\left(\varphi_{F_{1}}\right)}+\theta_{1}^{r}(D), 0\right)\right) \text { and } Q_{4}=Q_{3}+\left(0, \frac{b}{\nu_{r}\left(\varphi_{F_{1}}\right)}\right)
\end{gathered}
$$


when $\theta_{1}^{r}(D) \geq 0$ and $p_{r+1}$ is the satellite point $E_{\eta} \cap E_{r}$ (respectively, a free point). If $\theta_{1}^{r}(D)<0$ and $p_{r+1}$ is the satellite point $E_{\eta} \cap E_{r}$ (respectively, a free point), then the points which are contained in $\Delta_{\nu}(D)$ are

$$
\begin{gathered}
Q_{5}=\left(\frac{a \bar{\beta}_{g+1}\left(\nu_{r}\right)}{\nu_{r}\left(\varphi_{M_{0}}\right)}, \frac{a \nu_{r}\left(\varphi_{\eta}\right)}{\nu_{r}\left(\varphi_{M_{0}}\right)}\right)\left(\text { respectively, } Q_{5}=\left(\frac{a \bar{\beta}_{g+1}\left(\nu_{r}\right)}{\nu_{r}\left(\varphi_{M_{0}}\right)}, 0\right)\right), \\
Q_{6}=Q_{5}+\left(0, \frac{a}{\nu_{r}\left(\varphi_{M_{0}}\right)}\right), \\
Q_{7}=\left(\frac{(a+b \delta) \bar{\beta}_{g+1}\left(\nu_{r}\right)-\theta_{1}^{r}(D) \nu_{r}\left(\varphi_{M_{0}}\right)}{\nu_{r}\left(\varphi_{M_{0}}\right)+\delta \nu_{r}\left(\varphi_{F_{1}}\right)}, \frac{(a+b \delta) \nu_{r}\left(\varphi_{\eta}\right)-\theta_{1}^{r}(D) \nu_{\eta}\left(\varphi_{M_{0}}\right)}{\nu_{r}\left(\varphi_{M_{0}}\right)+\delta \nu_{r}\left(\varphi_{F_{1}}\right)}\right) \\
\left(\text { respectively, } Q_{7}=\left(\frac{(a+b \delta) \bar{\beta}_{g+1}\left(\nu_{r}\right)-\theta_{1}^{r}(D) \nu_{r}\left(\varphi_{M_{0}}\right)}{\nu_{r}\left(\varphi_{M_{0}}\right)+\delta \nu_{r}\left(\varphi_{F_{1}}\right)}, 0\right)\right) \\
\text { and } Q_{8}=Q_{7}+\left(0, \frac{a+b \delta}{\nu_{r}\left(\varphi_{M_{0}}\right)+\delta \nu_{r}\left(\varphi_{F_{1}}\right)}\right) .
\end{gathered}
$$

The point $Q_{9}=\left(\hat{\mu}_{D}\left(\nu_{r}\right), \hat{\mu}_{D}\left(\nu_{\eta}\right)\right)$ (respectively, $\left.Q_{9}=\left(\hat{\mu}_{D}\left(\nu_{r}\right), 0\right)\right)$ if $p_{r+1}$ is satellite (respectively, free) is also contained in $\Delta_{\nu}(D)$ by definition. This last point can be explicitly computed using Theorem 3.2.1.

Remark 3.3.20. In this remark, we give some observations about the previous points $Q_{i}$. They are the following ones.

(a) The value $\theta_{1}^{r}(D)$ vanishes if and only if $Q_{1}=Q_{3}\left(=Q_{5}=Q_{7}\right.$ when $\left.\nu_{r}\left(\varphi_{M_{0}}\right) \neq 0\right)$ and $Q_{2}=Q_{4}\left(=Q_{6}=Q_{8}\right.$ when $\left.\nu_{r}\left(\varphi_{M_{0}}\right) \neq 0\right)$. Moreover, if $\theta_{1}^{r}(D)<0$, it holds that $\delta>0$ and $a=0$ if and only if $Q_{5}=(0,0)=Q_{6}$.

(b) Some of the above points $Q_{i}$ are collinear by Lemma 3.3.6 and Lemma 3.3.19: Indeed,

(b.1) Assume that $p_{r+1}$ is the satellite point $E_{\eta} \cap E_{r}, \eta \neq r$. The points $(0,0), Q_{2}, Q_{4}$ (respectively, $\left.Q_{6}, Q_{8}\right)$ and $Q_{9}$ are in the line $T_{1} \equiv \bar{\beta}_{0}\left(\nu_{r}\right) y=$ $\bar{\beta}_{0}\left(\nu_{\eta}\right) x$ when $\eta \preccurlyeq r$ and $\theta_{1}^{r}(D) \geq 0$ (respectively, $\theta_{1}^{r}(D)<0$ ). If $\eta \npreceq r$ and $\theta_{1}^{r}(D) \geq 0$ (respectively, $\theta_{1}^{r}(D)<0$ ), then $(0,0), Q_{1}, Q_{3}$ (respectively, $\left.Q_{5}, Q_{7}\right)$ and $Q_{9}$ are in $T_{1}$.

(b.2) Suppose that $p_{r+1}$ is a free point. The points $(0,0), Q_{1}, Q_{3}$ (respectively, $\left.Q_{5}, Q_{7}\right)$ and $Q_{9}$ are in the line $y=0$.

Let us state our main result for the Case A.

Theorem 3.3.21. Let $\nu$ be an exceptional curve valuation. Assume that $\nu$ belongs to Case A defined before Lemma 3.3.19. Following the notations of the above paragraphs, the Newton-Okounkov body $\Delta_{\nu}(D)$ of a big and nef divisor $D \sim a F+b M$ on $\mathbb{F}_{\delta}$ with respect to $\nu$ is a quadrilateral if and only if $a \neq 0$ and $\theta_{1}^{r}(D) \neq 0$. Otherwise, it is a triangle (because one of the conditions of Remark 3.3.20(a) happens).

The vertices of $\Delta_{\nu}(D)$ are 
(a) $(0,0), Q_{1}, Q_{3}$ (respectively, $Q_{5}, Q_{7}$ ) and $Q_{9}$ if $p_{r+1}$ is the satellite point $E_{\eta} \cap E_{r}$, $\eta \neq r, \eta \preccurlyeq r$ and $\theta_{1}^{r}(D) \geq 0$ (respectively, $\theta_{1}^{r}(D)<0$ ).

(b) $(0,0), Q_{2}, Q_{4}$ (respectively, $Q_{6}, Q_{8}$ ) and $Q_{9}$ if $p_{r+1}$ is the satellite point $E_{\eta} \cap E_{r}$, $\eta \neq r, \eta \npreceq r$ and $\theta_{1}^{r}(D) \geq 0$ (respectively, $\theta_{1}^{r}(D)<0$ ).

(c) $(0,0), Q_{2}, Q_{4}$ (respectively, $\left.Q_{6}, Q_{8}\right)$ and $Q_{9}$ if $p_{r+1}$ is a free point and $\theta_{1}^{r}(D) \geq 0$ (respectively, $\left.\theta_{1}^{r}(D)<0\right)$.

Proof. We first prove that the sets of points $\left\{(0,0), Q_{1}, Q_{2}, Q_{3}, Q_{4}, Q_{9}\right\}$ and $\{(0,0)$, $\left.Q_{5}, Q_{6}, Q_{7}, Q_{8}, Q_{9}\right\}$ generate convex hulls, denoted by $\Delta$ and $\Delta^{\prime}$ respectively, whose area is equal to $D^{2} / 2$.

We begin with $\Delta$. Consider the triangle $(0,0), Q_{1}$ and $Q_{2}$ (respectively, $Q_{3}, Q_{4}$ and $\left.Q_{9}\right)$. Its area is

$$
\frac{b^{2} \bar{\beta}_{g+1}\left(\nu_{r}\right)}{2 \nu_{r}\left(\varphi_{F_{1}}\right)^{2}}\left(\text { respectively, } \frac{b}{2 \nu_{r}\left(\varphi_{F_{1}}\right)}\left(\hat{\mu}_{D}\left(\nu_{r}\right)-\left(\frac{b}{\nu_{r}\left(\varphi_{F_{1}}\right)} \bar{\beta}_{g+1}\left(\nu_{r}\right)+\theta_{1}^{r}(D)\right)\right)\right) .
$$

Now take the parallelogram $Q_{1}, Q_{2}, Q_{3}$ and $Q_{4}$. It is immediate that its area is $\frac{b}{\nu_{r}\left(\varphi_{F_{1}}\right)} \theta_{1}^{r}(D)$. Therefore, summing the previous areas yields that of $\Delta$ which equals

$$
\frac{2 a b+b^{2} \delta}{2}=\frac{D^{2}}{2}
$$

Proceeding analogously with $\Delta^{\prime}$, we sum the area of the triangles with vertices $(0,0), Q_{5}$ and $Q_{6}$, and $Q_{7}, Q_{8}$ and $Q_{9}$ together with that of the trapezium with vertices $Q_{5}, Q_{6}, Q_{7}$ and $Q_{8}$. It is a simple matter to see that the areas of the triangles are $\frac{a^{2}}{2 \nu_{r}\left(\varphi_{M_{0}}\right)^{2}} \bar{\beta}_{g+1}\left(\nu_{r}\right)$ and

$$
\frac{a+b \delta}{2\left(\nu_{r}\left(\varphi_{M_{0}}\right)+\delta \nu_{r}\left(\varphi_{F_{1}}\right)\right)}\left(\hat{\mu}_{D}\left(\nu_{r}\right)-\left(\frac{(a+b \delta) \bar{\beta}_{g+1}\left(\nu_{r}\right)-\theta_{1}^{r}(D) \nu_{r}\left(\varphi_{M_{0}}\right)}{\nu_{r}\left(\varphi_{M_{0}}\right)+\delta \nu_{r}\left(\varphi_{F_{1}}\right)}\right)\right) .
$$

Now we consider the trapezium. The length of its parallel sides (generated by $Q_{5}$ and $Q_{6}$, and $Q_{7}$ and $\left.Q_{8}\right)$ and the distance between them are

$$
\begin{aligned}
& \frac{a}{\nu_{r}\left(\varphi_{M_{0}}\right)}, \frac{a+b \delta}{\nu_{r}\left(\varphi_{M_{0}}\right)+\delta \nu_{r}\left(\varphi_{F_{1}}\right)} \text { and } \\
& \frac{-\theta_{1}^{r}(D)\left(\delta \bar{\beta}_{g+1}\left(\nu_{r}\right)+\nu_{r}\left(\varphi_{M_{0}}\right)^{2}\right)}{\nu_{r}\left(\varphi_{M_{0}}\right)\left(\nu_{r}\left(\varphi_{M_{0}}\right)+\delta \nu_{r}\left(\varphi_{F_{1}}\right)\right)} .
\end{aligned}
$$

Then the area of the trapezium is

$$
\frac{-\theta_{1}^{r}(D)\left((2 a+b \delta) \nu_{r}\left(\varphi_{M_{0}}\right)+a \delta \nu_{r}\left(\varphi_{F_{1}}\right)\right)\left(\delta \bar{\beta}_{g+1}\left(\nu_{r}\right)+\nu_{r}\left(\varphi_{M_{0}}\right)^{2}\right)}{2 \nu_{r}\left(\varphi_{M_{0}}\right)^{2}\left(\nu_{r}\left(\varphi_{M_{0}}\right)+\delta \nu_{r}\left(\varphi_{F_{1}}\right)\right)^{2}} .
$$

Continuing the process, when we sum the previous areas, the first observation is that the coefficients of $\bar{\beta}_{g+1}\left(\nu_{r}\right)$ are cancelled. Therefore, it suffices to sum the following 
fractions

$$
\begin{aligned}
& \frac{(a+b \delta) \hat{\mu}_{D}\left(\nu_{r}\right)}{2\left(\nu_{r}\left(\varphi_{M_{0}}\right)+\delta \nu_{r}\left(\varphi_{F_{1}}\right)\right)}, \frac{\theta_{1}^{r}(D)(a+b \delta) \nu_{r}\left(\varphi_{M_{0}}\right)}{2\left(\nu_{r}\left(\varphi_{M_{0}}\right)+\delta \nu_{r}\left(\varphi_{F_{1}}\right)\right)^{2}} \text { and } \\
& \frac{-\theta_{1}^{r}(D) \nu_{r}\left(\varphi_{M_{0}}\right)^{2}\left((2 a+b \delta b) \nu_{r}\left(\varphi_{M_{0}}\right)+a \delta \nu_{r}\left(\varphi_{F_{1}}\right)\right)}{2 \nu_{r}\left(\varphi_{M_{0}}\right)^{2}\left(\nu_{r}\left(\varphi_{M_{0}}\right)+\delta \nu_{r}\left(\varphi_{F_{1}}\right)\right)^{2}}
\end{aligned}
$$

which provides the desired value $D^{2} / 2$.

If we look at the vertices of $\Delta$ and $\Delta^{\prime}$, some of the defining points of $\Delta$ and $\Delta^{\prime}$ can be removed depending on the assumptions by Remark 3.3.20(b). As a result, the Newton-Okounkov body $\Delta_{\nu}(D)$ is a triangle or a quadrilateral.

To conclude the proof we are going to see that $\Delta_{\nu}(D)$ is a triangle if and only if one of the situations of Remark 3.3.20 (a) happens.

Suppose, for instance, that $p_{r+1}$ is a satellite point and $\eta \npreceq r$. The remaining cases run similarly. Take $\theta_{1}^{r}(D) \geq 0$. Here the Newton-Okounkov body $\Delta_{\nu}(D)$ is a triangle if and only if one of the following conditions holds: either the line going through $Q_{2}$ and $Q_{9}$ contains $Q_{4}$, or the point $Q_{4}$ is in the line $T_{2} \equiv \bar{\beta}_{g^{*}+1}\left(\nu_{\eta}\right) x=\bar{\beta}_{g^{*}+1}\left(\nu_{r}\right) y$. Both options are true if and only if $Q_{2}=Q_{4}$, or equivalently, $\theta_{1}^{r}(D)=0$. Now consider $\theta_{1}^{r}(D)<0$. In this situation, the Newton-Okounkov body $\Delta_{\nu}(D)$ is a triangle if and only if one of the following conditions happens: the line passing through $Q_{6}$ and $Q_{9}$ contains $Q_{8}$; the point $Q_{8}$ is in the line $T_{2} ;$ or $Q_{5}=(0,0)=Q_{6}$. As above, the first and second conditions are satisfied if and only if $\theta_{1}^{r}(D)=0$, which contradicts our assumption $\left(\theta_{1}^{r}(D)<0\right)$. By Remark 3.3.20, the third one holds if and only if $\delta>0$ and $a=0$, which completes the proof.

Let us show an example of Newton-Okounkov body $\Delta_{\nu}(D)$ which corresponds to Theorem 3.3.21 (a).

Example 3.3.22. Let $\nu_{r}$ be a special divisorial valuation of $\mathbb{F}_{\delta}$ (centered at $\mathcal{O}_{\mathbb{F}_{2}, p}$ ), where $p$ is a special point of $\mathbb{F}_{2}$, and $\left\{\bar{\beta}_{i}\left(\nu_{r}\right)\right\}_{i=0}^{3}=\{20,28,153,612\}$ its sequence of maximal contact values. Set $\mathcal{C}_{\nu_{r}}=\left\{p_{i}\right\}_{i=1}^{12}$ (with $p=p_{1}$ ) the configuration of infinitely near points of $\nu$ and write $F_{1}$ the fiber containing $p$. Assume that the strict transform of $M_{0}$ only passes through $p_{2}$. Therefore, $\nu_{r}\left(\varphi_{F_{1}}\right)=20, \nu_{r}\left(\varphi_{M_{0}}\right)=28$ and $2 \nu_{r}\left(\varphi_{F_{1}}\right) \nu_{r}\left(\varphi_{M_{0}}\right)+\nu_{r}\left(\varphi_{F_{1}}\right)^{2} \delta=1920>612=\bar{\beta}_{g+1}\left(\nu_{r}\right)$. That is, $\nu_{r}$ is non-positive at infinity by Theorem 2.3.7.

Set $\nu=\nu_{E_{\bullet}}$ the valuation defined by the flag $E_{\bullet}=\left\{Z=Z_{12} \supset E_{12} \supset\left\{p_{13}\right\}\right\}$, where $p_{13} \in E_{8} \cap E_{12}$, and whose first coordinate is the above divisorial valuation $\nu_{r}$. Following Theorem 3.3.21, the Newton-Okounkov body $\Delta_{\nu}(F+2 M)$ is a quadrilateral and its vertices are

$$
\mathbf{0}=(0,0), Q_{5}=\left(\frac{612}{28}, \frac{152}{28}\right), Q_{7}=\left(\frac{4068}{68}, \frac{1012}{68}\right) \text { and } Q_{9}=(156,39),
$$

since $\nu_{r}$ is non-minimal with respect to $F+2 M$ by Corollary 3.2.4, $\theta_{1}^{r}(F+2 M)<0$ and $8 \preccurlyeq 12$. Figure 3.3 shows the Newton-Okounkov body $\Delta_{\nu}(F+2 M)$ (in dark) and the triangle $\mathfrak{C}(\nu) \cap \mathfrak{H}_{F+2 M}(\nu)$ given in Proposition 3.3.5. 


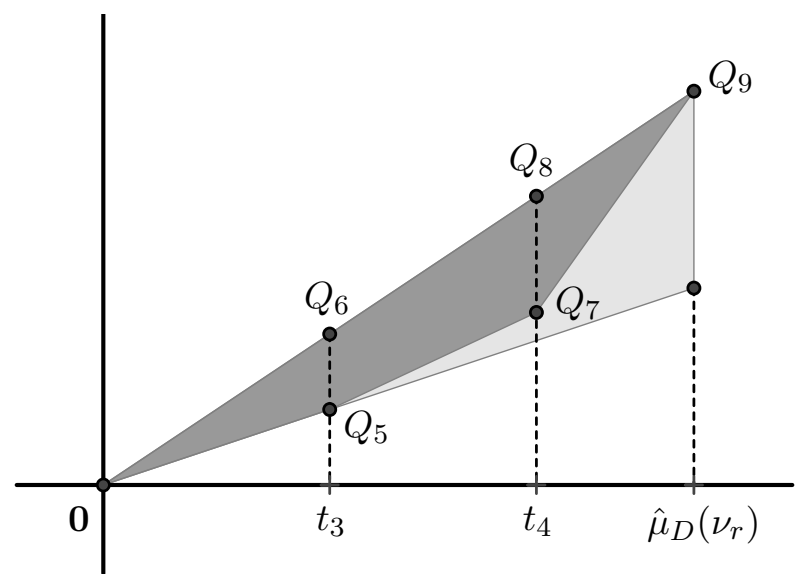

Figure 3.3: $\Delta_{\nu}(F+2 M)$ and $\mathfrak{C}(\nu) \cap \mathfrak{H}_{F+2 M}(\nu)$ in Example 3.3.22.

Next we formulate a similar result to Theorem 3.3.21 for Case B described before Lemma 3.3.19. That is, we assume that $\nu$ is a valuation in Case $\mathrm{B}$ and then $g^{*}=0$ and $\nu\left(\varphi_{F_{1}}\right)=\bar{\beta}_{1}(\nu)$. As the first point in $\mathbb{F}_{\delta}$ where we blow up can be special or general, it may happen that $\nu\left(\varphi_{M_{0}}\right)=(0,0)$. In this last case the value $\theta_{1}^{r}(D)=a \nu_{r}\left(\varphi_{F_{1}}\right)$ is non-negative. Moreover, we can suppose that $D$ is big and nef, since $\Delta_{\nu}(D)$ with $D$ big and not nef can be obtained as we said in the paragraphs before Subsection 3.3.1.

Notice that $\Delta_{\nu}(D)$ can also be described as the convex set (3.9). So, using Proposition 3.3.17, when $p_{r+1}$ is the satellite point $E_{\eta} \cap E_{r}$ and $\theta_{1}^{r}(D) \geq 0$ (respectively, $\theta_{1}^{r}(D)<0$ ), the points $Q_{1}, Q_{2}, Q_{3}, Q_{4}$ (respectively, $\left.Q_{5}, Q_{6}, Q_{7}, Q_{8}\right)$ and $Q_{9}$ given in (3.10) (respectively, (3.11)) for the satellite case belong to $\Delta_{\nu}(D)$. Otherwise $\left(p_{r+1}\right.$ is free), the points

$$
\begin{gathered}
Q_{1}=\left(\frac{b \bar{\beta}_{g+1}\left(\nu_{r}\right)}{\nu_{r}\left(\varphi_{F_{1}}\right)}, 0\right), Q_{2}=Q_{1}+\left(0, \frac{b}{\nu_{r}\left(\varphi_{F_{1}}\right)}\right) \\
Q_{3}=\left(\frac{b \bar{\beta}_{g+1}\left(\nu_{r}\right)}{\nu_{r}\left(\varphi_{F_{1}}\right)}+\theta_{1}^{r}(D), \frac{\theta_{1}^{r}(D)}{\nu_{r}\left(\varphi_{F_{1}}\right)}\right) \text { and } Q_{4}=Q_{3}+\left(0, \frac{b}{\nu_{r}\left(\varphi_{F_{1}}\right)}\right)
\end{gathered}
$$

(respectively, $Q_{5}, Q_{6}, Q_{7}, Q_{8}$ given in (3.11) for the free case) and

$$
Q_{9}=\left(\hat{\mu}_{D}\left(\nu_{r}\right), a+b \delta\right)
$$

are in $\Delta_{\nu}(D)$ if $\theta_{1}^{r}(D) \geq 0$ (respectively, $\theta_{1}^{r}(D)<0$ ).

Before determining the vertices of the Newton-Okounkov body in Case B, we show some situations where the previous points are aligned.

Remark 3.3.23. As in Case A, the points $Q_{i}$ described in the last but one paragraph satisfy the following properties.

(a) The statement in Remark 3.3.20(a) remains true. 
(b) Assume that $\nu\left(\varphi_{M_{0}}\right)=(0,0)$. It follows from Lemma 3.3.6 and Lemma 3.3.19 that the points $(0,0), Q_{1}, Q_{3}$ (respectively, $\left.Q_{2}, Q_{4}\right)$ and $Q_{9}$ are in the line $\bar{\beta}_{g^{*}+1}\left(\nu_{\eta}\right) x=\bar{\beta}_{g^{*}+1}\left(\nu_{r}\right) y$ when $p_{r+1}$ is the satellite point $E_{\eta} \cap E_{r}, \eta \neq r$, and $\eta \preccurlyeq r$ (respectively, $\eta \npreceq r$ ), since $\hat{\mu}_{D}\left(\nu_{r}\right)=a \nu_{r}\left(\varphi_{F_{1}}\right)$ and we are in Case B. If $p_{r+1}$ is a free point, then $(0,0), Q_{2}, Q_{4}$ and $Q_{9}$ belong to the line $y=x / \bar{\beta}_{g+1}\left(\nu_{r}\right)$.

(c) Assume $\nu\left(\varphi_{M_{0}}\right) \neq(0,0)$. As a consequence of Lemma 3.3.6 and Lemma 3.3.19, some of the before described points $Q_{i}$ are collinear. Indeed,

(c.1) Suppose that $p_{r+1}$ is the satellite point $E_{\eta} \cap E_{r}, \eta \neq r$. The points $(0,0), Q_{1}$ and $Q_{3}$ (respectively, $(0,0), Q_{6}$ and $\left.Q_{8}\right)$ belong to the line $T_{2} \equiv$ $\bar{\beta}_{g^{*}+1}\left(\nu_{r}\right) y=\bar{\beta}_{g^{*}+1}\left(\nu_{\eta}\right) x$ (respectively, $\left.T_{1} \equiv \bar{\beta}_{0}\left(\nu_{r}\right) y=\bar{\beta}_{0}\left(\nu_{\eta}\right) x\right)$, and the point $Q_{4}$ (respectively, $Q_{7}$ ) is contained in the line which passes through $Q_{2}$ and $Q_{9}$ (respectively, $Q_{5}$ and $Q_{9}$ ), when $\eta \preccurlyeq r$ and $\theta_{1}^{r}(D) \geq 0$ (respectively, $\left.\theta_{1}^{r}(D)<0\right)$.

(c.2) If $p_{r+1} \in E_{\eta} \cap E_{r}, \eta \neq r, \eta \npreceq r$ and $\theta_{1}^{r}(D) \geq 0$ (respectively, $\theta_{1}^{r}(D)<0$ ), then the points $(0,0), Q_{2}$ and $Q_{4}$ (respectively, $(0,0), Q_{5}$ and $\left.Q_{7}\right)$ are in the previous line $T_{2}$ (respectively, $T_{1}$ ), and $Q_{3}$ (respectively, $Q_{8}$ ) belongs to the line which goes through $Q_{1}$ and $Q_{9}$ (respectively, $Q_{6}$ and $Q_{9}$ ).

(c.3) Otherwise ( $p_{r+1}$ is a free point), the points $(0,0), Q_{2}$ and $Q_{4}$ (respectively, $(0,0), Q_{5}$ and $\left.Q_{7}\right)$ are in the line $y=x / \bar{\beta}_{g+1}\left(\nu_{r}\right)$ (respectively, $y=0$ ), and $Q_{3}$ (respectively, $Q_{8}$ ) is contained in the line which passes through $Q_{1}$ and $Q_{9}$ (respectively, $Q_{6}$ and $Q_{9}$ ) when $\theta_{1}^{r}(D) \geq 0$ (respectively, $\theta_{1}^{r}(D)<0$ ).

Theorem 3.3.24. Let $\nu$ be an exceptional curve valuation in Case B described before Lemma 3.3.19. With notations as in the previous paragraphs, the Newton-Okounkov body $\Delta_{\nu}(D)$ of a big and nef divisor $D \sim a F+b M$ on $\mathbb{F}_{\delta}$ with respect to $\nu$ is a quadrilateral if and only if $a \neq 0$. Otherwise, it is a triangle (see Remark 3.3.23).

(a) When $\nu\left(\varphi_{M_{0}}\right)=(0,0)$, the vertices of the quadrilateral are

(a.1) $(0,0), Q_{2}, Q_{4}$ (respectively, $\left.Q_{1}, Q_{3}\right)$ and $Q_{9}$ if $p_{r+1}$ is the satellite point $E_{\eta} \cap E_{r}, \eta \neq r$, and $\eta \preccurlyeq r$, (respectively, $\eta \nprec r$ ).

(a.2) $(0,0), Q_{1}, Q_{3}$ and $Q_{9}$ whenever $p_{r+1}$ is a free point.

In addition, if $\delta>0$ and $a=0$, then the vertices of the triangle $\Delta_{\nu}(D)$ are the above ones, where $Q_{1}=Q_{3}$ and $Q_{2}=Q_{4}$.

(b) When $\nu\left(\varphi_{M_{0}}\right) \neq(0,0)$, the vertices of the quadrilateral are

(b.1) $(0,0), Q_{2}, Q_{3}$ (respectively, $Q_{5}, Q_{8}$ ) and $Q_{9}$ if $\theta_{1}^{r}(D) \geq 0$ (respectively, $\left.\theta_{1}^{r}(D)<0\right), p_{r+1}$ is the satellite point $E_{\eta} \cap E_{r}, \eta \neq r$, and $\eta \preccurlyeq r$. 
(b.2) $(0,0), Q_{1}, Q_{4}$ (respectively, $\left.Q_{6}, Q_{7}\right)$ and $Q_{9}$ if $\theta_{1}^{r}(D) \geq 0$ (respectively, $\left.\theta_{1}^{r}(D)<0\right), p_{r+1}$ is the satellite point $E_{\eta} \cap E_{r}, \eta \neq r$, and $\eta \npreceq r$.

(b.3) $(0,0), Q_{1}, Q_{4}$ (respectively, $Q_{6}, Q_{7}$ ) and $Q_{9}$ if $\theta_{1}^{r}(D) \geq 0$ (respectively, $\left.\theta_{1}^{r}(D)<0\right)$ and $p_{r+1}$ is a free point.

Moreover, if $\delta>0$ and $a=0$, the vertices of the triangle $\Delta_{\nu}(D)$ are the above ones where $Q_{5}=(0,0)=Q_{6}$.

Proof. Take the convex hulls defined by the points $\left\{(0,0), Q_{1}, Q_{2}, Q_{3}, Q_{4}, Q_{9}\right\}$ and $\left\{(0,0), Q_{5}, Q_{6}, Q_{7}, Q_{8}, Q_{9}\right\}$. Reasoning as in the proof of Theorem 3.3.21, we deduce that the area of both sets is $D^{2} / 2$. In addition, taking into account Remark 3.3.23, one gets (a). Finally, checking that $Q_{9}$ does not belong to neither the line $T_{1} \equiv$ $\bar{\beta}_{0}\left(\nu_{r}\right) y=\bar{\beta}_{0}\left(\nu_{\eta}\right) x$ nor the line $T_{2} \equiv \bar{\beta}_{g^{*}+1}\left(\nu_{r}\right) y=\bar{\beta}_{g^{*}+1}\left(\nu_{\eta}\right) x$, we obtain (b) by Remark 3.3.23, which proves the result.

Example 3.3.25. Let $\nu_{r}$ be a special divisorial valuation of $\mathbb{F}_{2}$ and $\left\{\bar{\beta}_{i}\left(\nu_{r}\right)\right\}_{i=0}^{2}=$ $\{2,5,10\}$ its sequence of maximal contact values. Set $\mathcal{C}_{\nu_{r}}=\left\{p_{i}\right\}_{i=1}^{4}$ the configuration of infinitely near points of $\nu_{r}$ such that $p_{1}$ is a special point. Moreover, assume that the strict transforms of $F_{1}$ (the fiber passing through $p_{1}$ ) go through $p_{2}$ and $p_{3}$. Therefore, $\nu_{r}\left(\varphi_{F_{1}}\right)=5$ and $\nu_{r}\left(\varphi_{M_{0}}\right)=2$ and then

$$
2 \nu_{r}\left(\varphi_{M_{0}}\right) \nu_{r}\left(\varphi_{F_{1}}\right)+\delta \nu_{r}\left(\varphi_{F_{1}}\right)^{2}=70>10=\bar{\beta}_{g+1}\left(\nu_{r}\right) .
$$

As a result, the divisorial valuation $\nu_{r}$ is non-positive at infinite.

Let $\nu=\nu_{E_{\bullet}}$ be the exceptional valuation associated to the flag $E_{\bullet}=\{Z=$ $\left.Z_{4} \supset E_{4} \supset\left\{p_{r+1}\right\}\right\}$ and whose first component is the last divisorial valuation $\nu_{r}$. In addition, suppose that $p_{r+1}$ is the satellite point $E_{3} \cap E_{4}$. Then, its sequence of maximal contact values is $\left\{\bar{\beta}_{0}(\nu), \bar{\beta}_{1}(\nu)\right\}=\{(2,1),(5,3)\}, \nu\left(\varphi_{F_{1}}\right)=(5,3)=\bar{\beta}_{1}(\nu)$ and $\nu\left(\varphi_{M_{0}}\right)=(2,1)$. As a result, we are in Case B. Assume that $D=F+M$. By Theorem 3.2.1, $\hat{\mu}_{D}\left(\nu_{r}\right)=(a+b \delta) \nu_{r}\left(\varphi_{F_{1}}\right)+b \nu_{r}\left(\varphi_{M_{0}}\right)=17$ and so $\nu$ is non-minimal respect to $D$ (since $\hat{\mu}_{D}\left(\nu_{r}\right)^{2}>D^{2} \bar{\beta}_{g+1}\left(\nu_{r}\right)$ ). Therefore, by Theorem 3.3.24, the Newton-Okounkov body $\Delta_{\nu}(D)$ is the convex hull generated by

$$
(0,0), Q_{1}=(2,1), Q_{4}=(5,3) \text { and } Q_{9}=(17,10),
$$

because $\theta_{1}^{r}(D)=a \nu_{r}\left(\varphi_{F_{1}}\right)-b \nu_{r}\left(\varphi_{M_{0}}\right)=3$ and $3=\eta \npreceq r=4$. One can see in Figure 3.4 the Newton-Okounkov $\Delta_{\nu}(F+M)$ in dark and the triangle $\mathfrak{C}(\nu) \cap \mathfrak{H}_{F+M}(\nu)$ given in Proposition 3.3.5.

To conclude this subsection, we describe the Newton-Okounkov body $\Delta_{\nu}(D)$ in Case $\mathrm{C}$ introduced before Lemma 3.3.19. Therefore, assume that $g^{*}=0$ and $\nu\left(\varphi_{M_{0}}\right)=\bar{\beta}_{1}(\nu)$. Here, we can suppose that $D$ is a big and nef divisor except for the case when all the points $\left\{p_{i}\right\}_{i=1}^{r+1}$ are free. In this last situation, $p_{r+1} \in \operatorname{supp}\left(N_{D^{*}}\right)$ if and only if $D$ is big and not nef. 


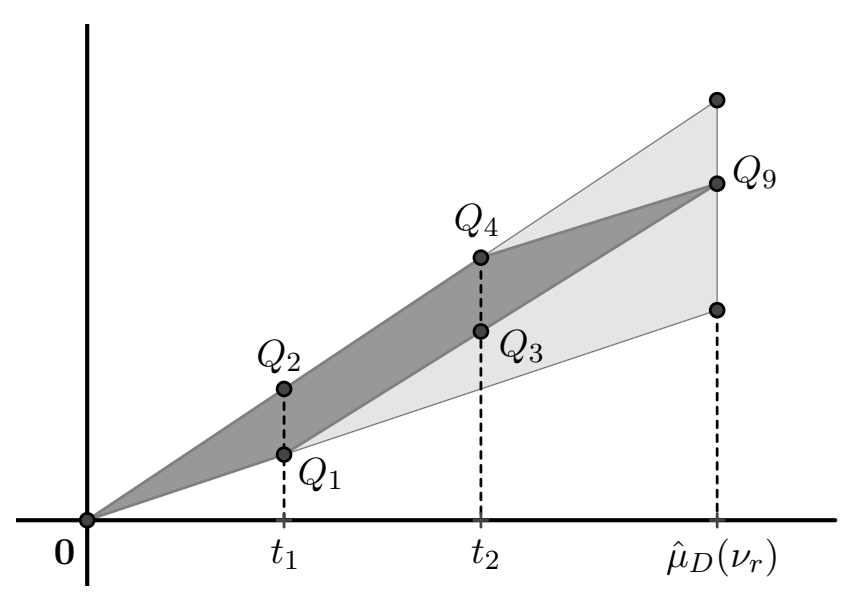

Figure 3.4: $\Delta_{\nu}(F+M)$ and $\mathfrak{C}(\nu) \cap \mathfrak{H}_{F+M}(\nu)$ in Example 3.3.25.

Let us start assuming that $D$ is big and nef. As above in Case $\mathrm{B}, \Delta_{\nu}(D)$ can be described as in (3.9). So, by Proposition 3.3.17, when $\theta_{1}^{r}(D) \geq 0$ (respectively, $\left.\theta_{1}^{r}(D)<0\right)$ and $p_{r+1}$ is the satellite point $E_{\eta} \cap E_{r}$, the points $Q_{1}, Q_{2}, Q_{3}, Q_{4}$ (respectively, $Q_{5}, Q_{6}, Q_{7}, Q_{8}$ ) and $Q_{9}$ provided in (3.10) (respectively, (3.11)) for the satellite situation belong to $\Delta_{\nu}(D)$.

If $p_{r+1}$ is a free point and $\theta_{1}^{r}(D)<0$ (respectively, $\theta_{1}^{r}(D) \geq 0$ ), the points

$$
\begin{gathered}
Q_{5}=\left(\frac{a \bar{\beta}_{g+1}\left(\nu_{r}\right)}{\nu_{r}\left(\varphi_{M_{0}}\right)}, 0\right), Q_{6}=Q_{5}+\left(0, \frac{a}{\nu_{r}\left(\varphi_{M_{0}}\right)}\right) \\
Q_{7}=\left(\frac{(a+b \delta) \bar{\beta}_{g+1}\left(\nu_{r}\right)-\theta_{1}^{r}(D) \nu_{r}\left(\varphi_{M_{0}}\right)}{\nu_{r}\left(\varphi_{M_{0}}\right)+\delta \nu_{r}\left(\varphi_{F_{1}}\right)}, \frac{-\theta_{1}^{r}(D)}{\nu_{r}\left(\varphi_{M_{0}}\right)+\delta \nu_{r}\left(\varphi_{F_{1}}\right)}\right) \\
Q_{8}=Q_{7}+\left(0, \frac{a+b \delta}{\nu_{r}\left(\varphi_{M_{0}}\right)+\delta \nu_{r}\left(\varphi_{F_{1}}\right)}\right)
\end{gathered}
$$

(respectively, $Q_{1}, Q_{2}, Q_{3}, Q_{4}$ given in (3.10) for the free case) and $Q_{9}=\left(\hat{\mu}_{D}\left(\nu_{r}\right), b\right)$ are in $\Delta_{\nu}(D)$.

Finally, assume that $D$ is big and not nef and all the points in $\left\{p_{i}\right\}_{i=1}^{r+1}$ are free. Recall that these hypothesis are equivalent to the fact that $p_{r+1} \in \operatorname{supp}\left(N_{D^{*}}\right)$ (see the paragraphs before Subsection 3.3.1). Therefore, the points

$$
\begin{gathered}
P_{1}=\left(\frac{-a \nu_{r}\left(\varphi_{M_{0}}\right)}{\delta}, \frac{-a}{\delta}\right), \\
P_{2}=\left(\frac{(a+b \delta) \bar{\beta}_{g+1}\left(\nu_{r}\right)-\theta_{1}^{r}(D) \nu_{r}\left(\varphi_{M_{0}}\right)}{\nu_{r}\left(\varphi_{M_{0}}\right)+\delta \nu_{r}\left(\varphi_{F_{1}}\right)}, \frac{-\theta_{1}^{r}(D)}{\nu_{r}\left(\varphi_{M_{0}}\right)+\delta \nu_{r}\left(\varphi_{F_{1}}\right)}\right), \\
P_{3}=P_{2}+\left(0, \frac{a+b \delta}{\nu_{r}\left(\varphi_{M_{0}}\right)+\delta \nu_{r}\left(\varphi_{F_{1}}\right)}\right) \text { and } P_{4}=\left(\hat{\mu}_{D}\left(\nu_{r}\right), b\right)
\end{gathered}
$$

belong to $\Delta_{\nu}(D)$. 
Remark 3.3.26. As the above cases, the points $Q_{i}, 1 \leq i \leq 9$, and $P_{j}, 1 \leq j \leq 4$, satisfy the following properties.

(a) The statement in Remark 3.3.20(a) holds if $D$ is a big and nef divisor.

(b) When $D$ is big and nef, some points $Q_{i}$ are collinear by Lemma 3.3.6 and Lemma 3.3.19. Indeed,

(b.1) If $p_{r+1}$ is the satellite point $E_{\eta} \cap E_{r}, \eta \neq r, \eta \preccurlyeq r$ and $\theta_{1}^{r}(D) \geq 0$ (respectively, $\left.\theta_{1}^{r}(D)\right)$, then the points $(0,0), Q_{2}$ and $Q_{4}$ (respectively, $(0,0), Q_{5}$ and $\left.Q_{7}\right)$ are in the line $T_{1} \equiv \bar{\beta}_{0}\left(\nu_{r}\right) y=\bar{\beta}_{0}\left(\nu_{\eta}\right) x$ (respectively, $T_{2} \equiv$ $\left.\bar{\beta}_{g^{*}+1}\left(\nu_{r}\right) y=\bar{\beta}_{g^{*}+1}\left(\nu_{\eta}\right) x\right)$, and the point $Q_{3}$ (respectively, $Q_{8}$ ) belongs to the line which goes through $Q_{1}$ and $Q_{9}$ (respectively, $Q_{6}$ and $Q_{9}$ ).

(b.2) When $p_{r+1} \in E_{\eta} \cap E_{r}, \eta \neq r, \eta \npreceq r$ and $\theta_{1}^{r}(D) \geq 0$ (respectively, $\theta_{1}^{r}(D)<$ $0)$, the points $(0,0), Q_{1}$ and $Q_{3}$ (respectively, $(0,0), Q_{6}$ and $\left.Q_{8}\right)$ are in the line $T_{1}$ (respectively, $T_{2}$ ) and the point $Q_{4}$ (respectively, $Q_{7}$ ) is contained in the line which passes through $Q_{2}$ and $Q_{9}$ (respectively, $Q_{5}$ and $Q_{9}$ ).

(b.3) Otherwise $\left(p_{r+1}\right.$ is a free point), the points $(0,0), Q_{1}$ and $Q_{3}$ (respectively, $(0,0), Q_{6}$ and $\left.Q_{8}\right)$ are in the line $y=0$ (respectively, $\left.y=x / \bar{\beta}_{g+1}\left(\nu_{r}\right)\right)$ and the point $Q_{4}$ (respectively, $Q_{7}$ ) belongs to the line which passes through $Q_{2}$ and $Q_{9}$ (respectively, $Q_{5}$ and $Q_{9}$ ).

(c) If $p_{r+1} \in \operatorname{supp}\left(N_{D^{*}}\right)$, then $\theta_{1}^{r}(D)<0$ and the point $P_{2}$ is contained in the line which goes through $P_{1}$ and $P_{4}$, and $P_{1}$ and $P_{3}$ belong to the line $y=x / \bar{\beta}_{g+1}\left(\nu_{r}\right)$.

Now, we formulate our result for Case $\mathrm{C}$ describing the vertices of the NewtonOkounkov body $\Delta_{\nu}(D)$. Notice that, as said, $D$ is big and nef except when $p_{r+1}$ is in $\operatorname{supp}\left(N_{D^{*}}\right)$. Recall that the Newton-Okounkov bodies $\Delta_{\nu}(D)$ for the remaining cases where $D$ is big but not nef can be reduced to the big and nef situation (see the paragraphs before Subsection 3.3.1).

Theorem 3.3.27. Let $\nu$ be an exceptional curve valuation as described in Case $C$ before Lemma 3.3.19. With assumptions and notations as in the previous paragraphs, the Newton-Okounkov body $\Delta_{\nu}(D)$ of a big divisor $D \sim a F+b M$ on $\mathbb{F}_{\delta}$ with respect to $\nu$ is a quadrilateral if and only if $a \neq 0$ and $D$ is nef. Otherwise, it is a triangle.

(a) When $D$ is a big and nef divisor, then the vertices of the quadrilateral are

(a.1) $(0,0), Q_{1}, Q_{4}$ (respectively, $Q_{6}, Q_{7}$ ) and $Q_{9}$ if $\theta_{1}^{r}(D) \geq 0$ (respectively, $\left.\theta_{1}^{r}(D)<0\right), p_{r+1}$ is the satellite point $E_{\eta} \cap E_{r}, \eta \neq r$, and $\eta \preccurlyeq r$.

(a.2) $(0,0), Q_{2}, Q_{3}$ (respectively, $\left.Q_{5}, Q_{8}\right)$ and $Q_{9}$ if $\theta_{1}^{r}(D) \geq 0$ (respectively, $\left.\theta_{1}^{r}(D)<0\right), p_{r+1}$ is the satellite point $E_{\eta} \cap E_{r}, \eta \neq r$, and $\eta \npreceq r$.

(a.3) $(0,0), Q_{2}, Q_{3}$ (respectively, $Q_{5}, Q_{8}$ ) and $Q_{9}$ if $\theta_{1}^{r}(D) \geq 0$ (respectively, $\left.\theta_{1}^{r}(D)<0\right)$ and $p_{r+1}$ is a free point. 
Moreover, if $\delta>0$ and $a=0$, the vertices of the triangle $\Delta_{\nu}(D)$ are the above ones where $Q_{5}=(0,0)=Q_{6}$.

(b) If $D$ is big but not nef and all the points in $\left\{p_{i}\right\}_{i=1}^{r+1}$ are free, then the vertices of the triangle $\Delta_{\nu}(D)$ are $P_{1}, P_{3}$ and $P_{4}$.

Proof. It easy see that (a) follows as in Theorem 3.3.24 (b) using Lemma 3.3.6 and Lemma 3.3.19.

Let us prove (b). Firstly, we are going to see that the area of the convex hull $\Delta$ generated by $P_{1}, P_{2}, P_{3}$ and $P_{4}$ is $P_{D^{*}}^{2} / 2$. Indeed, the area of the triangle generated by $P_{1}, P_{2}$ and $P_{3}$ (respectively, $P_{2}, P_{3}$ and $P_{4}$ ) is

$$
\begin{gathered}
\frac{(a+b \delta)\left(\frac{(a+b \delta) \bar{\beta}_{g+1}\left(\nu_{r}\right)-\theta_{1}^{r}(D) \nu_{r}\left(\varphi_{M_{0}}\right)}{\nu_{r}\left(\varphi_{M_{0}}\right)+\delta \nu_{r}\left(\varphi_{F_{1}}\right)}-\frac{-a \nu_{r}\left(\varphi_{M_{0}}\right)}{\delta}\right)}{2\left(\nu_{r}\left(\varphi_{M_{0}}\right)+\delta \nu_{r}\left(\varphi_{F_{1}}\right)\right)} \\
\left(\text { respectively, } \frac{(a+b \delta)\left(\hat{\mu}_{D}\left(\nu_{r}\right)-\frac{(a+b \delta) \bar{\beta}_{g+1}\left(\nu_{r}\right)-\theta_{1}^{r}(D) \nu_{r}\left(\varphi_{M_{0}}\right)}{\nu_{r}\left(\varphi_{M_{0}}\right)+\delta \nu_{r}\left(\varphi_{F_{1}}\right)}\right)}{2\left(\nu_{r}\left(\varphi_{M_{0}}\right)+\delta \nu_{r}\left(\varphi_{F_{1}}\right)\right)}\right) .
\end{gathered}
$$

Summing both areas, we have

$$
\begin{aligned}
\frac{(a+b \delta)\left(\hat{\mu}_{D}\left(\nu_{r}\right)-\frac{-a \nu_{r}\left(\varphi_{M_{0}}\right)}{\delta}\right)}{2\left(\nu_{r}\left(\varphi_{M_{0}}\right)+\delta \nu_{r}\left(\varphi_{F_{1}}\right)\right)} & =\frac{(a+b \delta)\left(b+\frac{a}{\delta}\right)\left(\nu_{r}\left(\varphi_{M_{0}}\right)+\delta \nu_{r}\left(\varphi_{F_{1}}\right)\right)}{2\left(\nu_{r}\left(\varphi_{M_{0}}\right)+\delta \nu_{r}\left(\varphi_{F_{1}}\right)\right)} \\
& =\frac{\left(\left(b+\frac{a}{\delta}\right) M^{*}\right)^{2}}{2}=\frac{P_{D^{*}}^{2}}{2}
\end{aligned}
$$

which is the desired value. After taking account Remark 3.3.26(c), the proof is concluded.

Example 3.3.28. Let $\nu_{r}$ be a special divisorial valuation of the Hirzebruch surface $\mathbb{F}_{3}$ and $\mathcal{C}_{\nu_{r}}=\left\{p_{i}\right\}_{i=1}^{3}$ its configuration of infinitely near point, where all points are free and $p_{1}$ is special. Assume that the strict transforms of the special section $M_{0}$ pass through $p_{2}$ and $p_{3}$. Consequently, $\nu_{r}\left(\varphi_{F_{1}}\right)=1, \nu_{r}\left(\varphi_{M_{0}}\right)=3$ and

$$
2 \nu_{r}\left(\varphi_{M_{0}}\right) \nu_{r}\left(\varphi_{F_{1}}\right)+\delta \nu_{r}\left(\varphi_{F_{1}}\right)^{2}=9>3=\bar{\beta}_{g+1}\left(\nu_{r}\right) .
$$

Thus $\nu_{r}$ is non-positive at infinity.

Set $\nu=\nu_{E_{\bullet}}$ the valuation associated to the flag $E_{\bullet}:=\left\{Z=Z_{3} \supset E_{3} \supset\left\{p_{r+1}\right\}\right\}$, where $p_{r+1}$ is a free point, such that its first component is the above divisorial valuation $\nu_{r}$. The sequence of maximal contact values of $\nu$ is $\left\{\bar{\beta}_{0}\left(\nu_{r}\right), \bar{\beta}_{1}\left(\nu_{r}\right)\right\}=$ $\{(1,0),(3,1)\}$. Assume that the strict transform of $M_{0}$ passes through $p_{r+1}$. Consider the divisor $D=-2 F+M$, which is big and not nef. Therefore, we are in Case C. By Theorem 3.2.1, $\hat{\mu}_{D}\left(\nu_{r}\right)=(a+b \delta) \nu_{r}\left(\varphi_{F_{1}}\right)+b \nu_{r}\left(\varphi_{M_{0}}\right)=4$ and then $\nu$ is 
non-minimal respect to $D$ (because $\hat{\mu}_{D}\left(\nu_{r}\right)^{2}>D^{2} \bar{\beta}_{g+1}\left(\nu_{r}\right)$ ). In addition, $\theta_{1}^{r}(D)=$ $a \nu_{r}\left(\varphi_{F_{1}}\right)-b \nu_{r}\left(\varphi_{M_{0}}\right)=-5$.

Then, by Theorem 3.3.27, the Newton-Okounkov body $\Delta_{\nu}(D)$ is the convex set whose vertices are

$$
P_{1}=\left(2, \frac{2}{3}\right), P_{3}=(3,1) \text { and } P_{4}=(4,1)
$$

Figure 3.5 shows the Newton-Okounkov body $\Delta_{\nu}(-2 F+M)$ in dark and the triangle $\mathfrak{C}(\nu) \cap \mathfrak{H}_{F+M}(\nu)$ described in Proposition 3.3.5.

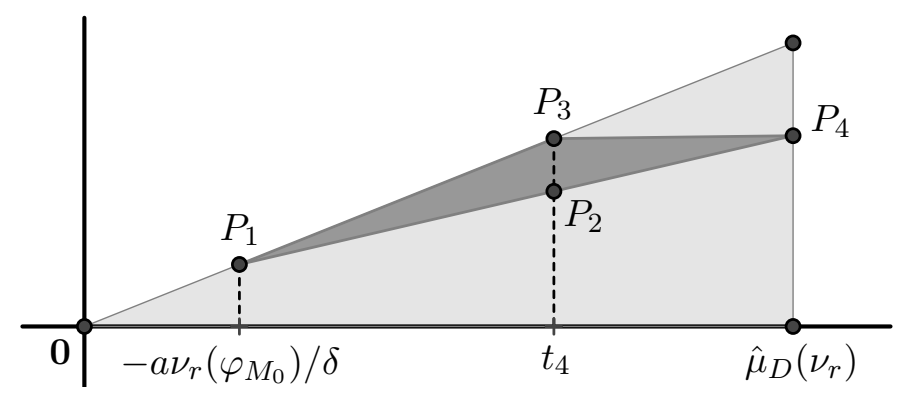

Figure 3.5: $\Delta_{\nu}(-2 F+M)$ and $\mathfrak{C}(\nu) \cap \mathfrak{H}_{-2 F+M}(\nu)$ in Example 3.3.28.

Corollary 3.3.29. Let $E_{\bullet}=\left\{Z=Z_{r} \supset E_{r} \supset\left\{p_{r+1}\right\}\right\}$ be a flag and $\nu=\nu_{E_{\bullet}}$ its attached exceptional curve valuation. Assume that its first component $\nu_{r}$ is an NPI divisorial valuation of $\mathbb{P}^{2}$ and thus $Z$ is obtained from a sequence of points blowups starting at $\mathbb{P}^{2}$. Then there exists an exceptional curve valuation $\hat{\nu}$ whose first component $\hat{\nu}_{r}$ is an NPI special divisorial valuation of $\mathbb{F}_{1}$ which corresponds to the same flag. Moreover, if $E_{0}$ is a general projective line on $\mathbb{P}^{2}$ and $M$ a curve of degree $(0,1)$ on $\mathbb{F}_{1}$, the Newton-Okounkov body $\Delta_{\nu}\left(E_{0}\right)$ of $E_{0}$ coincides with the Newton-Okounkov body $\Delta_{\hat{\nu}}(M)$ of $M$.

Proof. The first part is a consequence of Theorem 1.3.2(b), Proposition 2.3.3 and Corollary 2.3.4.

Now we are going to prove the second part of the result. Set $\left\{\bar{\beta}_{i}(\nu)\right\}_{i=0}^{g^{*}+1}$ (respectively, $\left.\left\{\bar{\beta}_{i}\left(\nu_{r}\right)\right\}_{i=0}^{g+1},\left\{\bar{\beta}_{i}(\hat{\nu})\right\}_{i=0}^{\hat{g}^{*}+1},\left\{\bar{\beta}_{i}\left(\hat{\nu}_{r}\right)\right\}_{i=0}^{\hat{g}+1}\right)$ the sequence of maximal contact values of $\nu$ (respectively, $\left.\nu_{r}, \hat{\nu}, \hat{\nu}_{r}\right)$ and $\nu_{\eta}$ (respectively, $\left.\hat{\nu}_{\hat{\eta}}\right)$ the divisorial valuation which appears in the second coordinate of $\nu$ (respectively, $\hat{\nu}$ ) when $p_{r+1}$ is a satellite point. Notice that $\hat{\eta}=\eta-1$.

Note that a projective line on $\mathbb{P}^{2}$ corresponds with a curve of degree $(0,1) D=M$ on $\mathbb{F}_{1}$. Therefore one always has that $\theta_{1}^{r}(D)=-\hat{\nu}_{r}\left(\varphi_{M_{0}}\right)<0$. Denote by $L$ the line at infinity on $\mathbb{P}^{2}$ (see Subsection 2.1). By the Noether formula, Proposition 2.3.3, 
Corollary 2.3.4, Remark 2.3.5 and Remark 3.2.2 the following equalities hold

$$
\begin{gathered}
\bar{\beta}_{g+1}\left(\nu_{r}\right)=\bar{\beta}_{\hat{g}+1}\left(\hat{\nu}_{r}\right)+\hat{\nu}_{r}\left(\varphi_{M_{0}}\right)^{2}, \nu\left(\mathfrak{m}_{1}\right)=\hat{\nu}\left(\varphi_{M_{0}}\right), \nu\left(\varphi_{L}\right)=\hat{\nu}\left(\varphi_{F_{1}}\right)+\hat{\nu}\left(\varphi_{M_{0}}\right), \\
\nu_{r}\left(\varphi_{\eta}\right)=\hat{\nu}_{r}\left(\varphi_{\hat{\eta}}\right)+\hat{\nu}_{r}\left(\varphi_{M_{0}}\right) \cdot \hat{\nu}_{\hat{\eta}}\left(\varphi_{M_{0}}\right) \text { and } \hat{\mu}\left(\nu_{r}\right)=\hat{\mu}_{D}\left(\hat{\nu}_{r}\right) .
\end{gathered}
$$

We distinguish three situations to show the result. Case 1: either $g^{*} \neq 0$, or $g^{*}=0$ and $\nu\left(\varphi_{L}\right) \neq \bar{\beta}_{1}(\nu)$; Case $2: g^{*}=0, \nu\left(\varphi_{L}\right)=\bar{\beta}_{1}(\nu)$ and $i_{L}>2$; and Case 3: $g^{*}=0, \nu\left(\varphi_{L}\right)=\bar{\beta}_{1}(\nu)$ and $i_{L}=2$, where $i_{L}$ indicates the last point in the configuration of infinitely near points $\mathcal{C}_{\nu_{r}}$ of $\nu_{r}$ through which the strict transform of $L$ passes.

Let us see Case 1. Consider the points given in (3.11) for Case A corresponding to the divisor $D=M$, the value $\theta_{1}^{r}(D)<0$ and $\delta=1$. That is, the points

$$
\begin{gathered}
\left.Q_{5}=(0,0)=Q_{6} \text { (respectively, } Q_{5}=(0,0)=Q_{6}\right), \\
Q_{7}=\left(\frac{\bar{\beta}_{g+1}\left(\hat{\nu}_{r}\right)+\hat{\nu}_{r}\left(\varphi_{M_{0}}\right)^{2}}{\hat{\nu}_{r}\left(\varphi_{M_{0}}\right)+\hat{\nu}_{r}\left(\varphi_{F_{1}}\right)}, \frac{\hat{\nu}_{r}\left(\varphi_{\hat{\eta}}\right)+\hat{\nu}_{r}\left(\varphi_{M_{0}}\right) \hat{\nu}_{\hat{\eta}}\left(\varphi_{M_{0}}\right)}{\hat{\nu}_{r}\left(\varphi_{M_{0}}\right)+\hat{\nu}_{r}\left(\varphi_{F_{1}}\right)}\right) \\
\left(\begin{array}{c}
\text { respectively, } \left.Q_{7}=\left(\frac{\bar{\beta}_{g+1}\left(\hat{\nu}_{r}\right)+\hat{\nu}_{r}\left(\varphi_{M_{0}}\right)^{2}}{\hat{\nu}_{r}\left(\varphi_{M_{0}}\right)+\hat{\nu}_{r}\left(\varphi_{F_{1}}\right)}, 0\right)\right), \\
Q_{8}=Q_{7}+\left(0, \frac{1}{\hat{\nu}_{r}\left(\varphi_{M_{0}}\right)+\hat{\nu}_{r}\left(\varphi_{F_{1}}\right)}\right) \text { and } Q_{9}=\left(\hat{\mu}_{D}\left(\hat{\nu}_{r}\right), \hat{\mu}_{D}\left(\hat{\nu}_{\hat{\eta}}\right)\right) \\
\left(\text { respectively, } Q_{9}=\left(\hat{\mu}_{D}\left(\hat{\nu}_{r}\right), 0\right)\right)
\end{array}\right.
\end{gathered}
$$

if $p_{r+1}$ is a satellite (respectively, free) point. Taking into account (3.12) and Theorems 3.3.10 and 3.3.21, we obtain the result for this case.

Notice that Case 2 and Case 3 can be proved with a similar reasoning and using the cases B and C (described before Lemma 3.3.19) and Theorems 3.3.24 and 3.3.27, respectively, which completes the proof.

Remark 3.3.30. The previous theorem provides two ways to describe the same Newton-Okounkov body, but the triangles given in Proposition 3.3.5 where it is contained are different. Notice that they correspond to distinct exceptional curve valuations.

Remark 3.3.31. Following the assumptions and notations of Corollary 3.3.29, the Newton-Okounkov body $\Delta_{\nu}\left(E_{0}\right)$ of a projective line $E_{0}$ on $\mathbb{P}^{2}$, where the valuation $\nu$ of $\mathbb{P}^{2}$ is minimal and non-positive at infinity, is described as the Newton-Okounkov body $\Delta_{\hat{\nu}}(M)$ of a curve of degree $(0,1) M$, where $\hat{\nu}$ is a non-minimal with respect to $M$ and special non-positive at infinity valuation of $\mathbb{F}_{1}$ (see Remark 3.2.2). Even more, this Newton-Okounkov body is the set given in Theorem 3.3.21 when $\delta=1, a=0$ and $\theta_{1}^{r}(D)<0$, since $\nu$ is minimal.

Example 3.3.32. Consider the first divisorial valuation $\nu_{n}$ of $\mathbb{P}^{2}$ described in Example 3.3.11. Recall that its configuration of infinitely near points is $\mathcal{C}_{\nu_{n}}=\left\{p_{i}\right\}_{i=1}^{17}$ 
and its sequence of maximal contact values is $\left\{\bar{\beta}_{i}\left(\nu_{n}\right)\right\}_{i=0}^{3}=\{8,20,63,256\}$. By Proposition 2.3.3 and Corollary 2.3.4, we can see the above divisorial valuation of $\mathbb{P}^{2}$ as the divisorial valuation $\nu_{r}$ of $\mathbb{F}_{1}$ whose sequence of maximal contact values is $\left\{\bar{\beta}_{i}\left(\nu_{r}\right)\right\}_{i=0}^{3}=\{8,12,47,192\}$.

In the first case of Example 3.3.11, we had that $\nu_{n}\left(\varphi_{L}\right)=16=\hat{\mu}\left(\nu_{n}\right)$ and then $\nu_{n}$ is non-positive at infinity and minimal. By the proof of Proposition 2.3.3, Theorem 3.2.1 and Remark 2.3.5, one gets that $\nu_{r}\left(\varphi_{F_{1}}\right)=8, \nu_{r}\left(\varphi_{M_{0}}\right)=8$ and $\hat{\mu}_{M}\left(\nu_{r}\right)=16$ and so $\nu_{r}$ is non-positive at infinity (by Remark 2.3.5) and non-minimal with respect to $M$ (since $256=\hat{\mu}_{D}\left(\nu_{r}\right)^{2}>M^{2} \bar{\beta}_{g+1}\left(\nu_{r}\right)=192$ ). Recall that $M$ is a curve of degree $(0,1)$ on $\mathbb{F}_{1}$. Taking into account Corollary 3.3.29, we can assume that $\nu_{r}$ is the first component of the valuation $\nu=\nu_{E_{\bullet}}$, where $E_{\bullet}$ is the flag $E_{\bullet}=\left\{Z_{17} \supset E_{17} \supset\left\{p_{r+1}\right\}\right\}$ defined in Example 3.3.11. If $p_{r+1}$ is the satellite point $E_{16} \cap E_{17}$ (with the notation of Example 3.3.11), the Newton-Okounkov body $\Delta_{\nu}(M)$ is the convex hull determined by the vertices

$$
(0,0), Q_{7}=\left(16, \frac{255}{16}\right) \text { and } Q_{9}=(16,16),
$$

since $\theta_{1}^{r}(M)=-8$ and $16 \preccurlyeq 17$. Otherwise $\left(p_{r+1}\right.$ is a free point $), \Delta_{\nu}(D)$ is triangle with vertices

$$
(0,0), Q_{8}=\left(16, \frac{1}{16}\right) \text { and } Q_{9}=(16,0) .
$$

Now assume that $\nu_{n}$ is the second divisorial valuation of $\mathbb{P}^{2}$ considered in Example 3.3.11. Here, we recall that $\nu_{n}\left(\varphi_{L}\right)=20=\hat{\mu}\left(\nu_{n}\right)$ and then $\nu_{n}$ is non-positive at infinity and non-minimal. With respect to the corresponding divisorial valuation $\nu_{r}$ of $\mathbb{F}_{1}$, one has $\nu_{r}\left(\varphi_{F_{1}}\right)=12, \nu_{r}\left(\varphi_{M_{0}}\right)=8$ and $\hat{\mu}_{M}\left(\nu_{r}\right)=20$ and then $\nu_{r}$ is nonpositive at infinity and non-minimal with respect to $M$. In this case, $\Delta_{\nu}(M)$ is described by the vertices

$$
(0,0), Q_{7}=\left(\frac{256}{20}, \frac{255}{20}\right) \text { and } Q_{9}=(20,20)
$$

since $p_{r+1}$ is the satellite point $E_{16} \cap E_{17}, 16 \preccurlyeq 17$ and $\theta_{1}^{r}(M)=-8$. Finally, if $p_{r+1}$ is a free point, the coordinates of $\Delta_{\nu}(M)$ are

$$
(0,0), Q_{8}=\left(\frac{256}{20}, \frac{1}{20}\right) \text { and } Q_{9}=(20,20) .
$$

\subsubsection{Newton-Okounkov bodies with respect to non-positive at in- finity non-special valuations}

To finish this chapter we complete our description of the Newton-Okounkov bodies associated to NPI valuations. It remains to study the case of non-special valuations which are non-minimal with respect to a big divisor on $\mathbb{F}_{\delta}$. We continue with the same notation introduced at the beginning of Section 3.3.

Let $\nu$ be an NPI non-special exceptional curve valuation of $\mathbb{F}_{\delta}$ whose first component is $\nu_{r}$. Set $D$ a big divisor on $\mathbb{F}_{\delta}$. We can assume that $D$ is also nef, since $p_{1}$ 
is general (see Definition 2.2.1) and then $p_{r+1} \notin \operatorname{supp}\left(N_{D^{*}}\right)$. Denote by $\theta_{2}^{r}(D)$ the expression $a \nu_{r}\left(\varphi_{F_{1}}\right)-b\left(\nu_{r}\left(\varphi_{M_{1}}\right)-\delta \nu_{r}\left(\varphi_{F_{1}}\right)\right)$, where $F_{1}$ is the fiber containing the point $p_{1}$ and $M_{1}$ the section described in Proposition 2.2.2. Notice that $\theta_{2}^{r}(D)=0$ if and only if $a=b\left(\nu_{r}\left(\varphi_{M_{1}}\right)-\delta \nu_{r}\left(\varphi_{F_{1}}\right)\right) / \nu_{r}\left(\varphi_{F_{1}}\right)$.

Lemma 3.3.33. Let $\nu_{r}$ be an NPI non-special divisorial valuation of $\mathbb{F}_{\delta}$. Consider a divisor $D$ and the value $\theta_{2}^{r}(D)$ as above. Then the divisor on the surface $Z$ defined by $\nu_{r}$

$$
D_{3}=D^{*}-\frac{b}{\nu_{r}\left(\varphi_{F_{1}}\right)} \sum_{i=1}^{r} \nu_{r}\left(\mathfrak{m}_{i}\right) E_{i}^{*}\left(\text { respectively, } D_{4}=D^{*}-\frac{a+b \delta}{\nu_{r}\left(\varphi_{M_{1}}\right)} \sum_{i=1}^{r} \nu_{r}\left(\mathfrak{m}_{i}\right) E_{i}^{*}\right)
$$

is nef if $\theta_{2}^{r}(D) \geq 0$ (respectively, $\left.\theta_{2}^{r}(D)<0\right)$.

Proof. Let us see that $D_{4}$ is a nef divisor if $\theta_{2}^{r}(D)<0$. The remaining case follows from a similar reasoning to that developed in Lemma 3.3.12.

Define

$$
\begin{gathered}
\Delta_{r}:=\left(\nu_{r}\left(\varphi_{M_{1}}\right)-\delta \nu_{r}\left(\varphi_{F_{1}}\right)\right) F^{*}+\nu_{r}\left(\varphi_{F_{1}}\right) M^{*}-\sum_{i=1}^{r} \nu_{r}\left(\mathfrak{m}_{i}\right) E_{i}^{*} \text { and } \\
\Gamma_{r}:=\nu_{r}\left(\varphi_{M_{1}}\right) M^{*}-\delta \sum_{i=1}^{r} \nu_{r}\left(\mathfrak{m}_{i}\right) E_{i}^{*} .
\end{gathered}
$$

Both divisors are nef by Theorem 2.3.7 and this finishes the proof because

$$
D_{4} \sim \frac{a}{\nu_{r}\left(\varphi_{M_{1}}\right)-\delta \nu_{r}\left(\varphi_{F_{1}}\right)} \Delta_{r}+\frac{-\theta_{2}^{r}(D)}{\nu_{r}\left(\varphi_{M_{1}}\right)-\delta \nu_{r}\left(\varphi_{F_{1}}\right)} \Gamma_{r}
$$

and $-\theta_{2}^{r}(D)>0$.

The next result can be proved as we did for Lemma 3.3.13. Recall that the non-positivity at infinity of a non-special divisorial valuation can be checked with the inequality $2 \nu_{r}\left(\varphi_{M_{1}}\right) \nu_{r}\left(\varphi_{F_{1}}\right)-\delta \nu_{r}\left(\varphi_{F_{1}}\right) \geq \bar{\beta}_{g+1}\left(\nu_{r}\right)$ (see Theorem 2.3.7). Besides, $D \sim a F+b M$ is a big and nef divisor on $\mathbb{F}_{\delta}$ and we also use the value $\theta_{2}^{r}(D)$.

Lemma 3.3.34. Let $\nu_{r}$ be an NPI non-special divisorial valuation of $\mathbb{F}_{\delta}$. Then the rational numbers

$$
\begin{gathered}
t_{5}=\frac{b}{\nu_{r}\left(\varphi_{F_{1}}\right)} \bar{\beta}_{g+1}\left(\nu_{r}\right) \text { and } t_{6}=\frac{b}{\nu_{r}\left(\varphi_{F_{1}}\right)} \bar{\beta}_{g+1}\left(\nu_{r}\right)+\theta_{2}^{r}(D) \\
\left(\text { respectively, } t_{7}=\frac{a+b \delta}{\nu_{r}\left(\varphi_{M_{1}}\right)} \bar{\beta}_{g+1}\left(\nu_{r}\right) \text { and } t_{8}=\frac{a \bar{\beta}_{g+1}\left(\nu_{r}\right)-\nu_{r}\left(\varphi_{M_{1}}\right) \theta_{2}^{r}(D)}{\nu_{r}\left(\varphi_{M_{1}}\right)-\delta \nu_{r}\left(\varphi_{F_{1}}\right)}\right)
\end{gathered}
$$

belong to the set $T_{D, \nu_{r}}:=\left\{t \in \mathbb{Q} \mid 0 \leq t \leq \hat{\mu}_{D}\left(\nu_{r}\right)\right\}$ when $\theta_{2}^{r}(D) \geq 0$ (respectively, $\left.\theta_{2}^{r}(D)<0\right)$.

Corollary 3.3.35. Let $\nu_{r}$ be an NPI non-special divisorial valuation of $\mathbb{F}_{\delta}$. Set $D \sim a F+b M a$ big and nef divisor on $\mathbb{F}_{\delta}$. Consider the value $\theta_{2}^{r}(D)$ given before Lemma 3.3 .33 and the rational numbers $t_{6}$ and $t_{8}$ provided in Lemma 3.3.34. Then 
(a) When $\theta_{2}^{r}(D) \geq 0$, it holds $2 \nu_{r}\left(\varphi_{M_{1}}\right) \nu_{r}\left(\varphi_{F_{1}}\right)-\delta \nu_{r}\left(\varphi_{F_{1}}\right)^{2}=\bar{\beta}_{g+1}\left(\nu_{r}\right)$ if and only if $t_{6}=\hat{\mu}_{D}\left(\nu_{r}\right)$.

(b) Otherwise $\left(\theta_{2}^{r}(D)<0\right)$, it holds either $2 \nu_{r}\left(\varphi_{M_{1}}\right) \nu_{r}\left(\varphi_{F_{1}}\right)-\delta \nu_{r}\left(\varphi_{F_{1}}\right)^{2}=\bar{\beta}_{g+1}\left(\nu_{r}\right)$, or $a=0$, if and only if $t_{8}=\hat{\mu}_{D}\left(\nu_{r}\right)$.

Proof. We only show (b), because (a) follows easily from the proof of Lemma 3.3.14. Let us start by considering that $2 \nu_{r}\left(\varphi_{M_{1}}\right) \nu_{r}\left(\varphi_{F_{1}}\right)+\delta \nu_{r}\left(\varphi_{F_{1}}\right)^{2}=\bar{\beta}_{g+1}\left(\nu_{r}\right)$. Applying the last condition to $t_{8}$, it holds

$$
\begin{aligned}
t_{8} & =\frac{a\left(2 \nu_{r}\left(\varphi_{M_{1}}\right) \nu_{r}\left(\varphi_{F_{1}}\right)-\delta \nu_{r}\left(\varphi_{F_{1}}\right)^{2}\right)-\nu_{r}\left(\varphi_{M_{1}}\right) \theta_{2}^{r}(D)}{\nu_{r}\left(\varphi_{M_{1}}\right)-\delta \nu_{r}\left(\varphi_{F_{1}}\right)} \\
& =\frac{\left(a \nu_{r}\left(\varphi_{F_{1}}\right)+b \nu_{r}\left(\varphi_{M_{1}}\right)\right)\left(\nu_{r}\left(\varphi_{M_{1}}\right)-\delta \nu_{r}\left(\varphi_{F_{1}}\right)\right)}{\nu_{r}\left(\varphi_{M_{1}}\right)-\delta \nu_{r}\left(\varphi_{F_{1}}\right)}=\hat{\mu}_{D}\left(\nu_{r}\right) .
\end{aligned}
$$

In addition, it is easy to check that $t_{8}$ equals $\hat{\mu}_{D}\left(\nu_{r}\right)$ when $a=0$.

Conversely, suppose that $t_{8}=\hat{\mu}_{D}\left(\nu_{r}\right)$. Then,

$$
\begin{aligned}
0=\hat{\mu}_{D}\left(\nu_{r}\right)-t_{8} & =\frac{\hat{\mu}_{D}\left(\nu_{r}\right)\left(\nu_{r}\left(\varphi_{M_{1}}\right)-\delta \nu_{r}\left(\varphi_{F_{1}}\right)\right)-a \bar{\beta}_{g+1}\left(\nu_{r}\right)+\nu_{r}\left(\varphi_{M_{1}}\right) \theta_{2}^{r}(D)}{\nu_{r}\left(\varphi_{M_{1}}\right)-\delta \nu_{r}\left(\varphi_{F_{1}}\right)} \\
& =\frac{a\left(2 \nu_{r}\left(\varphi_{M_{1}}\right) \nu_{r}\left(\varphi_{F_{1}}\right)+\delta \nu_{r}\left(\varphi_{F_{1}}\right)^{2}-\bar{\beta}_{g+1}\left(\nu_{r}\right)\right)}{\nu_{r}\left(\varphi_{M_{1}}\right)-\delta \nu_{r}\left(\varphi_{F_{1}}\right)}
\end{aligned}
$$

which completes the proof.

Remark 3.3.36. As in Remark 3.3.15, one can obtain information from the values $t_{i}, 5 \leq i \leq 8$.

(a) $\nu_{r}$ is minimal with respect to a big and nef divisor $D$ if and only if

$$
\hat{\mu}_{D}\left(\nu_{r}\right)=\frac{b \bar{\beta}_{g+1}\left(\nu_{r}\right)}{\nu_{r}\left(\varphi_{F_{1}}\right)}=t_{5}=t_{6}=\frac{(a+b \delta) \bar{\beta}_{g+1}\left(\nu_{r}\right)}{\nu_{r}\left(\varphi_{M_{1}}\right)}=t_{7}=t_{8} .
$$

In fact, the equalities $t_{5}=t_{6}$ and $\hat{\mu}_{D}\left(\nu_{r}\right)=t_{6}$ are equivalents to those provide in Corollary 3.2.3 by Corollary 3.3.35.

(b) Assume that $\nu_{r}$ is non-minimal with respect to $D$. Then,

(b.1) $\theta_{2}^{r}(D)=0$ if and only if $\hat{\mu}_{D}\left(\nu_{r}\right)>t_{5}=t_{6}=t_{7}=t_{8}>0$.

(b.2) If $\theta_{2}^{r}(D)>0,2 \nu_{r}\left(\varphi_{M_{1}}\right) \nu_{r}\left(\varphi_{F_{1}}\right)-\delta \nu_{r}\left(\varphi_{F_{1}}\right)^{2} \geq \bar{\beta}_{g+1}\left(\nu_{r}\right)$ if and only if $\hat{\mu}_{D}\left(\nu_{r}\right) \geq t_{6}>t_{5}>0$.

(b.3) If $\theta_{2}^{r}(D)<0,2 \nu_{r}\left(\varphi_{M_{1}}\right) \nu_{r}\left(\varphi_{F_{1}}\right)-\delta \nu_{r}\left(\varphi_{F_{1}}\right)^{2} \geq \bar{\beta}_{g+1}\left(\nu_{r}\right)$ and $a \geq 0$ if and only if $\hat{\mu}_{D}\left(\nu_{r}\right) \geq t_{8}>t_{7}>0$.

Arguing with the divisors $D_{3}$ and $D_{4}$ from Lemma 3.3.33 as we did with those from Lemma 3.3.16, one can show that $D_{3}$ and $D_{4}$ are also big. In addition,

$$
D_{3} \cdot \tilde{F}_{1}=0, D_{4} \cdot \tilde{M}_{1}=0 \text { and } D_{3} \cdot E_{i}=0 \text { and } D_{4} \cdot E_{i}=0
$$

for $1 \leq i \leq r-1$. As a result, one obtains the next lemma. 
Lemma 3.3.37. Let $\nu_{r}$ be a divisorial valuation and $D$ a divisor as in Corollary 3.3.35. Assume also that $\nu_{r}$ is non-minimal with respect to $D$. The intersection matrix determined by the set of divisors $\left\{\tilde{F}_{1}, E_{1}, E_{2}, \ldots, E_{r-1}\right\}$ (respectively, $\left\{\tilde{M}_{1}, E_{1}, E_{2}\right.$, $\left.\left.\ldots, E_{r-1}\right\}\right)$ is negative definite.

Let $\nu_{r}$ be a divisorial valuation and $D$ a divisor as stated in Corollary 3.3.35. Consider the rational numbers $t_{i}, 5 \leq i \leq 8$, defined in Lemma 3.3.34. The following proposition determines the Zariski decomposition of the divisors $D^{*}-t_{i} E_{r}$, which will use to describe the Newton-Okounkov bodies $\Delta_{\nu}(D)$ of exceptional curve valuations as before Lemma 3.3.33. Our determination depends on the above defined value $\theta_{2}^{r}(D)$ and the divisors $D_{3}, D_{4}$ and

$$
\Delta_{r}=\left(\nu_{r}\left(\varphi_{M_{1}}\right)-\delta \nu_{r}\left(\varphi_{F_{1}}\right)\right) F^{*}+\nu_{r}\left(\varphi_{F_{1}}\right) M^{*}-\sum_{i=1}^{r} \nu_{r}\left(\mathfrak{m}_{i}\right) E_{i}^{*}
$$

provided in Lemma 3.3.33 and its proof.

Proposition 3.3.38. The following statements hold.

(a) The positive and negative parts of the Zariski decomposition of the divisor $D_{t_{5}}=$ $D^{*}-t_{5} E_{r}$ (respectively, $D_{t_{6}}=D^{*}-t_{6} E_{r}$ ) are

$$
\begin{gathered}
P_{D_{t_{5}}} \sim D_{3} \text { and } N_{D_{t_{5}}}=\frac{b}{\nu_{r}\left(\varphi_{F_{1}}\right)} \sum_{i=1}^{r-1} \nu_{r}\left(\varphi_{i}\right) E_{i} \\
\left(\text { respectively, } P_{D_{t_{6}}} \sim \frac{b}{\nu_{r}\left(\varphi_{F_{1}}\right)} \Delta_{r}\right. \text { and } \\
\left.N_{D_{t_{6}}}=\frac{\theta_{2}^{r}(D)}{\nu_{r}\left(\varphi_{F_{1}}\right)} \tilde{F}_{1}+\sum_{i=1}^{r-1} \frac{b \nu_{r}\left(\varphi_{i}\right)+\theta_{2}^{r}(D) \nu_{i}\left(\varphi_{F_{1}}\right)}{\nu_{r}\left(\varphi_{F_{1}}\right)} E_{i}\right),
\end{gathered}
$$

when $\theta_{2}^{r}(D) \geq 0$.

(b) The positive and negative parts of the Zariski decomposition of $D_{t_{7}}=D^{*}-t_{7} E_{r}$ (respectively, $D_{t_{8}}=D^{*}-t_{8} E_{r}$ ) are

$$
\begin{gathered}
P_{D_{t_{7}}} \sim D_{4} \text { and } N_{D_{t_{7}}}=\frac{a+b \delta}{\nu_{r}\left(\varphi_{M_{1}}\right)} \sum_{i=1}^{r-1} \nu_{r}\left(\varphi_{i}\right) E_{i} \\
\left(\text { respectively, } P_{D_{t_{8}}} \sim \frac{a}{\nu_{r}\left(\varphi_{M_{1}}\right)-\delta \nu_{r}\left(\varphi_{F_{1}}\right)} \Delta_{r}\right. \text { and } \\
\left.N_{D_{t_{8}}}=\frac{-\theta_{2}^{r}(D)}{\nu_{r}\left(\varphi_{M_{1}}\right)-\delta \nu_{r}\left(\varphi_{F_{1}}\right)} \tilde{M}_{1}+\sum_{i=1}^{r-1} \frac{a \nu_{r}\left(\varphi_{i}\right)-\theta_{2}^{r}(D) \nu_{i}\left(\varphi_{M_{1}}\right)}{\nu_{r}\left(\varphi_{M_{1}}\right)-\delta \nu_{r}\left(\varphi_{F_{1}}\right)} E_{i}\right),
\end{gathered}
$$

when $\theta_{2}^{r}(D)<0$.

Proof. We are going to show (b). A proof for (a) follows similarly. On the one hand, the components of the divisor $N_{D_{t_{7}}}$ determine a negative definite intersection matrix. 
On the other hand, $P_{D_{t_{7}}}$ is a nef divisor by Lemma 3.3.33 and orthogonal to each component of $N_{D_{t_{7}}}$ by the proximity equalities. Therefore, $P_{D_{t_{7}}}+N_{D_{t_{7}}}$ is the Zariski decomposition of $D_{t_{7}}$.

Let us prove our statement for $D_{t_{8}}$. The components of $N_{D_{t_{8}}}$ determine a negative definite intersection matrix by Lemma 3.3.37 and $P_{D_{t_{8}}}$ is a nef divisor and orthogonal to each component of $N_{D_{t_{8}}}$ by Proposition 2.4.2 and Theorem 2.4.8. To finish, let us show that $P_{D_{t_{8}}}+N_{D_{t_{8}}} \sim D_{t_{8}}$, which finishes the proof. Indeed, set $p_{i_{M_{1}}}$ the last point in the configuration of infinitely near points $\mathcal{C}_{\nu_{r}}$ of the valuation $\nu_{r}$ through which the strict transform of $M_{1}$ passes. From the fact that $\tilde{M}_{1} \sim M^{*}-\sum_{i=1}^{i_{M_{1}}} E_{i}^{*}$, one deduces that

$$
\frac{a\left(\Delta_{r}+\sum_{i=1}^{r-1} \nu_{r}\left(\varphi_{i}\right) E_{i}\right)+\theta_{2}^{r}(D) M^{*}}{\nu_{r}\left(\varphi_{M_{1}}\right)-\delta \nu_{r}\left(\varphi_{F_{1}}\right)} \sim D-\frac{a \bar{\beta}_{g+1}\left(\nu_{r}\right)}{\nu_{r}\left(\varphi_{M_{1}}\right)-\delta \nu_{r}\left(\varphi_{F_{1}}\right)} E_{r} .
$$

Besides,

$$
\frac{-\theta_{2}^{r}(D)}{\nu_{r}\left(\varphi_{M_{1}}\right)-\delta \nu_{r}\left(\varphi_{M_{1}}\right)}\left(\sum_{i=1}^{r-1} \nu_{i}\left(\varphi_{M_{1}}\right) E_{i}-\sum_{i=1}^{i_{M_{1}}} E_{i}^{*}\right)=\frac{-\theta_{2}^{r}(D) \nu_{r}\left(\varphi_{M_{1}}\right)}{\nu_{r}\left(\varphi_{M_{1}}\right)-\delta \nu_{r}\left(\varphi_{F_{1}}\right)} E_{r}
$$

and the proof concludes after summing both expressions.

Remark 3.3.39. As in Remark 3.3.18, we can deduce from the above results that the ray $\left[D_{t}\right]:=\left[D^{*}\right]-t\left[E_{r}\right]$, where $0 \leq t \leq \hat{\mu}_{D}\left(\nu_{r}\right)$, crosses the interior of $\operatorname{NE}(Z)$ heading towards the face of $\mathrm{NE}(Z)$ spanned by the classes of the divisors $\tilde{F}_{1}, \tilde{M}_{1}, E_{1}, E_{2}, \ldots$, $E_{r-1}$. Figure 3.6 shows the situation in this case, where $\left[\Delta_{n}\right]^{\perp}$ represents the mentioned face of $\mathrm{NE}(Z)$ and the Zariski chambers $\Sigma_{1}, \Sigma_{2}, \Sigma_{3}$ and $\Sigma_{4}$ defined below are delimited by discontinuous lines.

$$
\begin{gathered}
\Sigma_{1}:=\left\{[\xi] \in \operatorname{Big}(Z) \mid \operatorname{Neg}(\xi)=\operatorname{Null}(\xi)=\left\{\left[E_{1}\right],\left[E_{2}\right], \ldots,\left[E_{r-1}\right]\right\}\right\}, \\
\Sigma_{2}:=\left\{[\xi] \in \operatorname{Big}(Z) \mid \operatorname{Neg}(\xi)=\operatorname{Null}(\xi)=\left\{\left[\tilde{F}_{1}\right],\left[E_{1}\right],\left[E_{2}\right], \ldots,\left[E_{r-1}\right]\right\}\right\}, \\
\Sigma_{3}:=\left\{[\xi] \in \operatorname{Big}(Z) \mid \operatorname{Neg}(\xi)=\operatorname{Null}(\xi)=\left\{\left[\tilde{M}_{1}\right],\left[E_{1}\right],\left[E_{2}\right], \ldots,\left[E_{r-1}\right]\right\}\right\} \text { and } \\
\Sigma_{4}:=\left\{[\xi] \in \operatorname{Big}(Z) \mid \operatorname{Neg}(\xi)=\operatorname{Null}(\xi)=\left\{\left[\tilde{F}_{1}\right],\left[\tilde{M}_{1}\right],\left[E_{1}\right],\left[E_{2}\right], \ldots,\left[E_{r-1}\right]\right\}\right\} .
\end{gathered}
$$

We finish this subsection describing the Newton-Okounkov bodies $\Delta_{\nu}(D)$, where $\nu$ and $D$ are as in the paragraph before Lemma 3.3.33. Recall that the first component of $\nu$ is the divisorial valuation $\nu_{r}$. As in the previous subsection, we divide our description in two cases.

Case D: Either $g^{*}>0$ or $g^{*}=0$ and $\nu\left(\varphi_{M_{1}}\right) \neq \bar{\beta}_{1}(\nu)$.

Case E: The value $g^{*}$ equals 0 and $\nu\left(\varphi_{M_{1}}\right)=\bar{\beta}_{1}(\nu)$.

Lemma 3.3.40. Let $\nu$ be an NPI non-special exceptional valuation of $\mathbb{F}_{\delta}$ and $\mathcal{C}_{\nu}=$ $\left\{p_{i}\right\}_{i \geq 1}$ its configuration of infinitely near points such that $p_{i} \rightarrow p_{r}$ for all $i>r$. Consider the cases described before. 


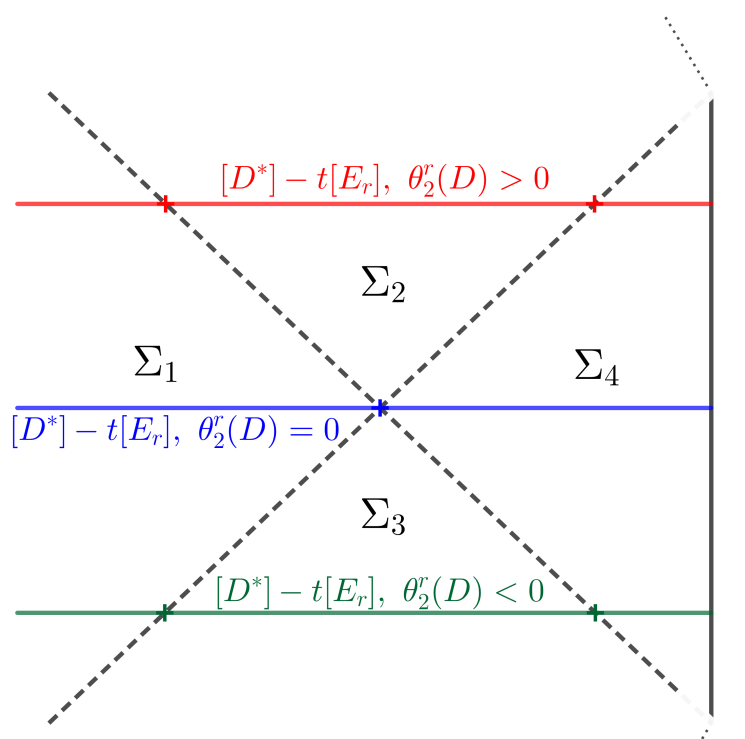

$\left[\Delta_{r}\right]^{\perp}$

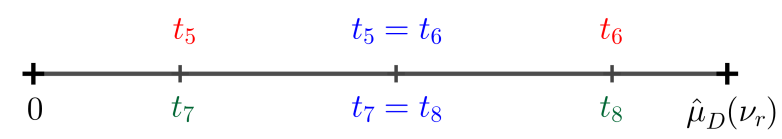

Figure 3.6: Local description of the cone of curves $\mathrm{NE}(Z)$ of a rational surface $Z$ given by a non-positive at infinity non-special divisorial valuation of $\mathbb{F}_{\delta}$.

(a) Suppose that $p_{r+1}$ is the satellite point $E_{\eta} \cap E_{r}, \eta \neq r$.

(a.1) Assume we are in Case D. Then,

$$
\nu_{\eta}\left(\varphi_{F_{1}}\right)=\nu_{r}\left(\varphi_{F_{1}}\right) \cdot \frac{\bar{\beta}_{0}\left(\nu_{\eta}\right)}{\bar{\beta}_{0}\left(\nu_{r}\right)} \text { and } \nu_{\eta}\left(\varphi_{M_{1}}\right)=\nu_{r}\left(\varphi_{M_{1}}\right) \cdot \frac{\bar{\beta}_{0}\left(\nu_{\eta}\right)}{\bar{\beta}_{0}\left(\nu_{r}\right)}
$$

(a.2) Assume we are in Case E. Then,

$$
\nu_{\eta}\left(\varphi_{F_{1}}\right)=\nu_{r}\left(\varphi_{F_{1}}\right) \cdot \frac{\bar{\beta}_{0}\left(\nu_{\eta}\right)}{\bar{\beta}_{0}\left(\nu_{r}\right)} \text { and } \nu_{\eta}\left(\varphi_{M_{1}}\right)=\nu_{r}\left(\varphi_{M_{1}}\right) \cdot \frac{\bar{\beta}_{g^{*}+1}\left(\nu_{\eta}\right)}{\bar{\beta}_{g^{*}+1}\left(\nu_{r}\right)} \text {. }
$$

(b) Otherwise ( $p_{r+1}$ is a free point).

(b.1) Assume we are in Case D. Then,

$$
\nu\left(\varphi_{F_{1}}\right)=\left(\nu_{r}\left(\varphi_{F_{1}}\right), 0\right) \text { and } \nu\left(\varphi_{M_{0}}\right)=\left(\nu_{r}\left(\varphi_{M_{0}}\right), 0\right)
$$

(b.2) Assume we are in Case E. Then,

$$
\nu\left(\varphi_{F_{1}}\right)=\left(\nu_{r}\left(\varphi_{F_{1}}\right), 0\right) \text { and } \nu\left(\varphi_{M_{0}}\right)=\left(\nu_{r}\left(\varphi_{M_{1}}\right), 1\right) \text {. }
$$


Proof. The result is immediate using the next properties: The value $\nu\left(\varphi_{F_{1}}\right)$ equals $\bar{\beta}_{0}(\nu)$ since the strict transform of $F_{1}$ does not go through $p_{2}$. Moreover, the value $\nu\left(\varphi_{M_{1}}\right)$ equals either $\bar{\beta}_{1}(\nu)$ when the strict transforms of $M_{1}$ pass through all initial free points of $\mathcal{C}_{\nu}$, or $s_{M_{1}} \bar{\beta}_{0}(\nu)$, where $s_{M_{1}}$ is a positive integer greater than $\delta$. This concludes the proof.

We begin our study with Case D. Reasoning as in the previous subsection, the Newton-Okounkov body $\Delta_{\nu}(D)$ can be described as in (3.9) and, by Proposition 3.3.38, the points

$$
\begin{gathered}
Q_{10}=\left(\frac{b \bar{\beta}_{g+1}\left(\nu_{r}\right)}{\nu_{r}\left(\varphi_{F_{1}}\right)}, \frac{b \nu_{r}\left(\varphi_{\eta}\right)}{\nu_{r}\left(\varphi_{F_{1}}\right)}\right)\left(\text { respectively, } Q_{10}=\left(\frac{b \bar{\beta}_{g+1}\left(\nu_{r}\right)}{\nu_{r}\left(\varphi_{F_{1}}\right)}, 0\right)\right), \\
Q_{11}=Q_{10}+\left(0, \frac{b}{\nu_{r}\left(\varphi_{F_{1}}\right)}\right), \\
Q_{12}=\left(\frac{b \bar{\beta}_{g+1}\left(\nu_{r}\right)}{\nu_{r}\left(\varphi_{F_{1}}\right)}+\theta_{2}^{r}(D), \frac{b \nu_{r}\left(\varphi_{\eta}\right)+\theta_{2}^{r}(D) \nu_{\eta}\left(\varphi_{F_{1}}\right)}{\nu_{r}\left(\varphi_{F_{1}}\right)}\right) \\
\left(\text { respectively } Q_{12}=\left(\frac{b \bar{\beta}_{g+1}\left(\nu_{r}\right)}{\nu_{r}\left(\varphi_{F_{1}}\right)}+\theta_{2}^{r}(D), 0\right)\right) \text { and } Q_{13}=Q_{12}+\left(0, \frac{b}{\nu_{r}\left(\varphi_{F_{1}}\right)}\right)
\end{gathered}
$$

are in $\Delta_{\nu}(D)$ if $\theta_{2}^{r}(D) \geq 0$ and $p_{r+1}$ is the satellite point $E_{\eta} \cap E_{r}$ (respectively, a free point).

When $\theta_{2}^{r}(D)<0$ and $p_{r+1} \in E_{\eta} \cap E_{r}$ (respectively, $p_{r+1}$ is a free point), the points in $\Delta_{\nu}(D)$ are

$$
\begin{gathered}
Q_{14}=\left(\frac{(a+b \delta) \bar{\beta}_{g+1}\left(\nu_{r}\right)}{\nu_{r}\left(\varphi_{M_{1}}\right)}, \frac{(a+b \delta) \nu_{r}\left(\varphi_{\eta}\right)}{\nu_{r}\left(\varphi_{M_{1}}\right)}\right) \\
\left(\text { respectively, } Q_{14}=\left(\frac{(a+b \delta) \bar{\beta}_{g+1}\left(\nu_{r}\right)}{\nu_{r}\left(\varphi_{M_{1}}\right)}, 0\right)\right), Q_{15}=Q_{14}+\left(0, \frac{a+b \delta}{\nu_{r}\left(\varphi_{M_{1}}\right)}\right) \\
Q_{16}=\left(\frac{a \bar{\beta}_{g+1}\left(\nu_{r}\right)-\theta_{2}^{r}(D) \nu_{r}\left(\varphi_{M_{1}}\right)}{\nu_{r}\left(\varphi_{M_{1}}\right)-\delta \nu_{r}\left(\varphi_{F_{1}}\right)}, \frac{a \nu_{r}\left(\varphi_{\eta}\right)-\theta_{2}^{r}(D) \nu_{\eta}\left(\varphi_{M_{1}}\right)}{\nu_{r}\left(\varphi_{M_{1}}\right)-\delta \nu_{r}\left(\varphi_{F_{1}}\right)}\right) \\
\left(\begin{array}{c}
\text { respectively, } Q_{16}=\left(\frac{a \bar{\beta}_{g+1}\left(\nu_{r}\right)-\theta_{2}^{r}(D) \nu_{r}\left(\varphi_{M_{1}}\right)}{\nu_{r}\left(\varphi_{M_{1}}\right)-\delta \nu_{r}\left(\varphi_{F_{1}}\right)}, 0\right) \\
a
\end{array}\right) \\
\text { and } Q_{17}=Q_{16}+\left(0, \frac{a}{\nu_{r}\left(\varphi_{M_{1}}\right)-\delta \nu_{r}\left(\varphi_{F_{1}}\right)}\right)
\end{gathered}
$$

In addition, the point $Q_{18}=\left(\hat{\mu}_{D}\left(\nu_{r}\right), \hat{\mu}_{D}\left(\nu_{\eta}\right)\right)$ (respectively, $\left.Q_{18}=\left(\hat{\mu}_{D}\left(\nu_{r}\right), 0\right)\right)$ belongs to $\Delta_{\nu}(D)$ when $p_{r+1}$ is satellite (respectively, free), by Theorem 3.2.1.

Remark 3.3.41. The latter points $Q_{i}, 10 \leq i \leq 18$, satisfy the following nice properties:

(a) $\theta_{2}^{r}(D)=0$ if and only if $Q_{10}=Q_{12}=Q_{14}=Q_{16}$ and $Q_{11}=Q_{13}=Q_{15}=Q_{17}$. Moreover, when $\theta_{2}^{r}(D)<0$, it holds that $a=0$ if and only if $Q_{16}=Q_{17}=Q_{18}$. 
(b) Some of the previous points $Q_{i}$ are collinear. Indeed,

(b.1) Consider $p_{r+1}$ the satellite point $E_{\eta} \cap E_{r}, \eta \neq r$, and $\eta \preccurlyeq r$. When $\theta_{2}^{r}(D) \geq 0$ (respectively, $\theta_{2}^{r}(D)<0$ ) the points $(0,0), Q_{11}, Q_{13}$ (respectively, $\left.Q_{15}, Q_{17}\right)$ and $Q_{18}$ are in the line $T_{1} \equiv \bar{\beta}_{0}\left(\nu_{r}\right) y=\bar{\beta}_{0}\left(\nu_{\eta}\right) x$. If $p_{r+1} \in E_{\eta} \cap E_{r}, \eta \npreceq r$, and $\theta_{2}^{r}(D) \geq 0$ (respectively, $\theta_{2}^{r}(D)<0$ ), the points $(0,0), Q_{10}, Q_{12}$ (respectively, $\left.Q_{14}, Q_{16}\right)$ and $Q_{18}$ also belong to $T_{1}$.

(b.2) Assume that $p_{r+1}$ is a free point. If $\theta_{2}^{r}(D) \geq 0$ (respectively, $\theta_{2}^{r}(D)<0$ ), the points $(0,0), Q_{10}, Q_{12}$ (respectively, $\left.Q_{14}, Q_{16}\right)$ and $Q_{18}$ are contained in the line $y=0$.

Theorem 3.3.42. Let $\nu$ be an exceptional valuation in Case D. Keeping the notation in the previous paragraphs, the Newton-Okounkov body $\Delta_{\nu}(D)$ of $D$ with respect to $\nu$ is a quadrilateral if and only if $a \neq 0$ and $\theta_{2}^{r}(D) \neq 0$. Otherwise, it is a triangle (see Remark 3.3.41).

The vertices of $\Delta_{\nu}(D)$ are

(a) $(0,0), Q_{10}, Q_{12}$ (respectively, $Q_{14}, Q_{16}$ ) and $Q_{18}$ when $\theta_{2}^{r}(D) \geq 0$ (respectively, $\left.\theta_{2}^{r}(D)<0\right), p_{r+1}$ is the satellite point $E_{\eta} \cap E_{r}, \eta \neq r$, and $\eta \preccurlyeq r$.

(b) $(0,0), Q_{11}, Q_{13}$ (respectively, $Q_{15}, Q_{17}$ ) and $Q_{18}$ when $\theta_{2}^{r}(D) \geq 0$ (respectively, $\left.\theta_{2}^{r}(D)<0\right), p_{r+1}$ is the satellite point $E_{\eta} \cap E_{r}, \eta \neq r$, and $\eta \npreceq r$.

(c) $(0,0), Q_{11}, Q_{13}$ (respectively, $Q_{15}, Q_{17}$ ) and $Q_{18}$ when $\theta_{2}^{r}(D) \geq 0$ (respectively, $\left.\theta_{2}^{r}(D)<0\right)$ and $p_{r+1}$ is a free point.

Proof. Let us see that $D^{2} / 2$ is the area of the convex hull $\Delta$ generated by the points $(0,0), Q_{14}, Q_{15}, Q_{16}, Q_{17}$ and $Q_{18}$. By Remark 3.3.41, the case concerning the points $(0,0), Q_{10}, Q_{11}, Q_{12}, Q_{13}$ and $Q_{18}$ and the fact of being a quadrilateral or a triangle follow as in the proof of Theorem 3.3.21.

The area of the triangle with vertices $(0,0), Q_{14}$ and $Q_{15}$ (respectively, $Q_{16}, Q_{17}$ and $\left.Q_{18}\right)$ is

$$
\begin{gathered}
\frac{(a+b \delta)^{2}}{2 \nu_{r}\left(\varphi_{M_{1}}\right)^{2}} \bar{\beta}_{g+1}\left(\nu_{r}\right)(\text { respectively, } \\
\left.\frac{a}{2\left(\nu_{r}\left(\varphi_{M_{1}}\right)-\delta \nu_{r}\left(\varphi_{F_{1}}\right)\right)}\left(\hat{\mu}_{D}\left(\nu_{r}\right)-\left(\frac{a \bar{\beta}_{g+1}\left(\nu_{r}\right)-\theta_{2}^{r}(D) \nu_{r}\left(\varphi_{M_{1}}\right)}{\nu_{r}\left(\varphi_{M_{1}}\right)-\delta \nu_{r}\left(\varphi_{F_{1}}\right)}\right)\right)\right) .
\end{gathered}
$$

The area of the trapezium provided by $Q_{14}, Q_{15}, Q_{16}$ and $Q_{17}$ is

$$
\frac{-\theta_{2}^{r}(D)\left((a+b \delta)\left(\nu_{r}\left(\varphi_{M_{1}}\right)-\delta \nu_{r}\left(\varphi_{F_{1}}\right)\right)+a \nu_{r}\left(\varphi_{F_{1}}\right)\right)\left(\nu_{r}\left(\varphi_{M_{0}}\right)^{2}-\delta \bar{\beta}_{g+1}\left(\nu_{r}\right)\right)}{2 \nu_{r}\left(\varphi_{M_{1}}\right)^{2}\left(\nu_{r}\left(\varphi_{M_{1}}\right)-\delta \nu_{r}\left(\varphi_{F_{1}}\right)\right)^{2}} .
$$


Summing the areas of that trapezium and the two previous triangles, we note that the coefficients of $\bar{\beta}_{g+1}\left(\nu_{r}\right)$ vanish and it is sufficient to sum the next three fractions

$$
\begin{gathered}
\frac{a \hat{\mu}_{D}\left(\nu_{r}\right)}{2\left(\nu_{r}\left(\varphi_{M_{1}}\right)-\delta \nu_{r}\left(\varphi_{F_{1}}\right)\right)}, \frac{a \theta_{2}^{r}(D) \nu_{r}\left(\varphi_{M_{1}}\right)}{2\left(\nu_{r}\left(\varphi_{M_{1}}\right)-\delta \nu_{r}\left(\varphi_{F_{1}}\right)\right)^{2}} \text { and } \\
\frac{-\theta_{2}^{r}(D) \nu_{r}\left(\varphi_{M_{1}}\right)^{2}\left((a+b \delta)\left(\nu_{r}\left(\varphi_{M_{1}}\right)-\delta \nu_{r}\left(\varphi_{F_{1}}\right)\right)+a \nu_{r}\left(\varphi_{F_{1}}\right)\right)}{2 \nu_{r}\left(\varphi_{M_{1}}\right)^{2}\left(\nu_{r}\left(\varphi_{M_{1}}\right)-\delta \nu_{r}\left(\varphi_{F_{1}}\right)\right)^{2}} .
\end{gathered}
$$

After computing, one obtains $\left(2 a b+\delta b^{2}\right) / 2$, which concludes the proof.

Example 3.3.43. Let $p$ be a general point of the Hirzebruch surface $\mathbb{F}_{2}$ and $\nu_{r}$ a non-special divisorial valuation centered at $\mathcal{O}_{\mathbb{F}_{2}, p}$, whose sequence of maximal contact values is $\left\{\bar{\beta}_{i}\left(\nu_{r}\right)\right\}_{i=0}^{3}=\{15,51,262,786\}$. Set $\mathcal{C}_{\nu_{r}}=\left\{p_{i}\right\}_{i=1}^{12}$ (with $p=p_{1}$ ) the configuration of infinitely near points of $\nu_{r}, F_{1}$ the fiber going through $p$ and $M_{1}$ the integral curve described in Proposition 2.2.2 whose strict transforms pass through $p_{2}$ and $p_{3}$. That is, the self-intersection of $\tilde{M}_{1}$ is negative. Therefore, $\nu_{r}\left(\varphi_{F_{1}}\right)=15$ and $\nu_{r}\left(\varphi_{M_{1}}\right)=45$ and then

$$
2 \nu_{r}\left(\varphi_{F_{1}}\right) \nu_{r}\left(\varphi_{M_{1}}\right)-\delta \nu_{r}\left(\varphi_{F_{1}}\right)^{2}=900>786=\bar{\beta}_{g+1}\left(\nu_{r}\right) .
$$

As a result, $\nu_{r}$ is non-positive at infinity by [63, Theorem 4.8].

Let $\nu=\nu_{E}$. be the valuation defined by the flag

$$
E_{\bullet}=\left\{Z=Z_{12} \supset E_{12} \supset\left\{p_{13}\right\}\right\},
$$

where $p_{13} \in E_{9} \cap E_{12}$, whose first component is the above divisorial valuation $\nu_{r}$. By Theorem 3.3.42, the coordinates of the vertices of the Newton-Okounkov body $\Delta_{\nu}(2 F+5 M)$ are

$$
(0,0), Q_{14}=\left(\frac{9432}{45}, \frac{3132}{45}\right), Q_{16}=\left(\frac{3597}{15}, \frac{1197}{15}\right) \text { and } Q_{18}=(255,85),
$$

because $\nu_{r}$ is non-minimal with respect to $2 F+5 M$ by Corollary 3.2.4, $\theta_{2}^{r}(D)<0$ and $9=\eta \preccurlyeq r=12$.

To finish, we study $\Delta_{\nu}(D)$ when $\nu$ is in Case E. As in Case D, $\Delta_{\nu}(D)$ can be seen as the set in (3.9) and then, by Proposition 3.3.38, the points $Q_{10}, Q_{11}, Q_{12}, Q_{13}$ (respectively, $Q_{14}, Q_{15}, Q_{16}, Q_{17}$ ) and $Q_{18}$ provided in (3.13) (respectively, (3.14)) for the satellite case are in $\Delta_{\nu}(D)$ when $p_{r+1}$ is the satellite point $E_{\eta} \cap E_{r}, \eta \neq r$, and $\theta_{2}^{r}(D) \geq 0$ (respectively, $\theta_{2}^{r}(D)<0$ ). If $p_{r+1}$ is a free point and $\theta_{2}^{r}(D)<0$ (respectively, $\theta_{2}^{r}(D) \geq 0$ ), the points

$$
\begin{gathered}
Q_{14}=\left(\frac{(a+b \delta) \bar{\beta}_{g+1}\left(\nu_{r}\right)}{\nu_{r}\left(\varphi_{M_{1}}\right)}, 0\right), Q_{15}=Q_{14}+\left(0, \frac{a+b \delta}{\nu_{r}\left(\varphi_{M_{1}}\right)}\right), \\
Q_{16}=\left(\frac{a \bar{\beta}_{g+1}\left(\nu_{r}\right)-\theta_{2}^{r}(D) \nu_{r}\left(\varphi_{M_{1}}\right)}{\nu_{r}\left(\varphi_{M_{1}}\right)-\delta \nu_{r}\left(\varphi_{F_{1}}\right)}, \frac{-\theta_{2}^{r}(D)}{\nu_{r}\left(\varphi_{M_{1}}\right)-\delta \nu_{r}\left(\varphi_{F_{1}}\right)}\right), \\
Q_{17}=Q_{16}+\left(0, \frac{a}{\nu_{r}\left(\varphi_{M_{1}}\right)-\delta \nu_{r}\left(\varphi_{F_{1}}\right)}\right)
\end{gathered}
$$


(respectively, $Q_{10}, Q_{11}, Q_{12}, Q_{13}$ given in (3.13) for the free case) and $\left.Q_{18}=\left(\hat{\mu}_{D}\left(\nu_{r}\right)\right), b\right)$ belong to $\Delta_{\nu}(D)$.

Remark 3.3.44. The previous points $Q_{i}, 1 \leq i \leq 18$, satisfy the following conditions.

(a) Remark 3.3.41(a) is also true for these points.

(b) Some of the above points $Q_{i}$ are collinear. Indeed,

(b.1) Assume that $p_{r+1}$ is the satellite point $E_{\eta} \cap E_{r}, \eta \neq r$, and $\eta \preccurlyeq r$. When $\theta_{2}^{r}(D) \geq 0$ (respectively, $\theta_{2}^{r}(D)$ ), the points $(0,0), Q_{11}$ and $Q_{13}$ (respectively, $(0,0), Q_{14}$ and $\left.Q_{16}\right)$ are in the line $T_{1} \equiv \bar{\beta}_{0}\left(\nu_{r}\right) y=\bar{\beta}_{0}\left(\nu_{\eta}\right) x$ (respectively, $\left.T_{2} \equiv \bar{\beta}_{g^{*}+1}\left(\nu_{r}\right) y=\bar{\beta}_{g^{*}+1}\left(\nu_{\eta}\right) x\right)$ and the point $Q_{12}$ (respectively, $Q_{17}$ ) belongs to the line which goes through $Q_{10}$ and $Q_{18}$ (respectively, $Q_{15}$ and $\left.Q_{18}\right)$.

(b.2) Suppose now that $p_{r+1} \in E_{\eta} \cap E_{r}, \eta \neq r$, and $\eta \npreceq r$. If $\theta_{2}^{r}(D) \geq 0$ (respectively, $\left.\theta_{2}^{r}(D)\right)$, the points $(0,0), Q_{10}$ and $Q_{12}$ (respectively, $(0,0), Q_{15}$ and $Q_{17}$ ) are contained in the line $T_{1} \equiv \bar{\beta}_{0}\left(\nu_{r}\right) y=\bar{\beta}_{0}\left(\nu_{\eta}\right) x$ (respectively, $\left.T_{2} \equiv \bar{\beta}_{g^{*}+1}\left(\nu_{r}\right) y=\bar{\beta}_{g^{*}+1}\left(\nu_{\eta}\right) x\right)$ and the point $Q_{13}$ (respectively, $\left.Q_{16}\right)$ is in the line which passes through $Q_{11}$ and $Q_{18}$ (respectively, $Q_{14}$ and $Q_{18}$ ).

(b.3) Assume that $p_{r+1}$ is a free point. If $\theta_{2}^{r}(D) \geq 0$ (respectively, $\theta_{2}^{r}(D)<0$ ), the points $(0,0), Q_{10}$ and $Q_{12}$ (respectively, $(0,0), Q_{15}$ and $Q_{17}$ ) belong to the line $y=0$ (respectively, $\left.y=x / \bar{\beta}_{g+1}\left(\nu_{r}\right)\right)$ and the point $Q_{13}$ (respectively, $Q_{16}$ ) is contained in the line going through $Q_{11}$ and $Q_{18}$ (respectively, $Q_{14}$ and $\left.Q_{18}\right)$.

Theorem 3.3.45. Let $\nu$ be an exceptional curve valuation in Case $E$ described before Lemma 3.3.40. Under the previous notations, the Newton-Okounkov body $\Delta_{\nu}(D)$ of $D$ with respect to $\nu$ is a quadrilateral if and only if $a \neq 0$. Otherwise, it is a triangle (see Remark 3.3.44).

The vertices of $\Delta_{\nu}(D)$ are

(a) $(0,0), Q_{10}, Q_{13}$ (respectively, $Q_{15}, Q_{16}$ ) and $Q_{18}$ if $\theta_{1}^{r}(D) \geq 0$ (respectively, $\left.\theta_{1}^{r}(D)<0\right), p_{r+1}$ is the satellite point $E_{\eta} \cap E_{r}, \eta \neq r$, and $\eta \preccurlyeq r$.

(b) $(0,0), Q_{11}, Q_{12}$ (respectively, $\left.Q_{14}, Q_{17}\right)$ and $Q_{18}$ if $\theta_{1}^{r}(D) \geq 0$ (respectively, $\left.\theta_{1}^{r}(D)<0\right), p_{r+1}$ is the satellite point $E_{\eta} \cap E_{r}, \eta \neq r$, and $\eta \npreceq r$.

(c) $(0,0), Q_{11}, Q_{12}$ (respectively, $Q_{14}, Q_{17}$ ) and $Q_{18}$ if $\theta_{1}^{r}(D) \geq 0$ (respectively, $\theta_{1}^{r}(D)<0$ ) and $p_{r+1}$ is a free point.

Proof. The result follows as in the proof of Theorem 3.3.42 to compute the area of the convex hulls generated by the points given in the statement, and as in Theorem 3.3.24 (b) after taking into account Remark 3.3.44. 
Example 3.3.46. Let $\nu_{r}$ be a non-special divisorial valuation of the Hirzebruch surface $\mathbb{F}_{4}$ and $\mathcal{C}_{\nu_{r}}=\left\{p_{i}\right\}_{i=1}^{5}$ the configuration of infinitely near points of $\nu_{r}$ where all the points are free. Assume that the strict transforms of $M_{1}$ go through $p_{2}, p_{3}, p_{4}$ and $p_{5}$. Then, $\nu_{r}\left(\varphi_{F_{1}}\right)=1, \nu_{r}\left(\varphi_{M_{0}}\right)=5$ and $2 \nu_{r}\left(\varphi_{M_{1}}\right) \nu_{r}\left(\varphi_{F_{1}}\right)-\delta \nu_{r}\left(\varphi_{F_{1}}\right)^{2}=6>$ $5=\bar{\beta}_{g+1}\left(\nu_{r}\right)$, and then $\nu_{r}$ is non-positive at infinity.

Set $\nu=\nu_{E_{\bullet}}$ the exceptional curve valuation associated to the flag $E_{\bullet}=\{Z=$ $\left.Z_{5} \supset E_{5} \supset\left\{p_{r+1}\right\}\right\}$, whose first component is the previous divisorial valuation $\nu_{r}$ and $p_{r+1}$ is a free point. The sequence of maximal contact values of $\nu$ is $\left\{\bar{\beta}_{0}(\nu), \bar{\beta}_{1}(\nu)\right\}$ $=\{(1,0),(5,1)\}$. Assume that the strict transform of $M_{1}$ goes through $p_{r+1}$, therefore we are in Case E. Consider the big and nef divisor $D=2 F+3 M$. By Theorem 3.2.1, $\hat{\mu}_{D}\left(\nu_{r}\right)=a \nu_{r}\left(\varphi_{F_{1}}\right)+b \nu_{r}\left(\varphi_{M_{0}}\right)=17$ and then $\nu$ is non-minimal with respect to $D$ (since $\hat{\mu}_{D}\left(\nu_{r}\right)>D^{2} \bar{\beta}_{g+1}\left(\nu_{r}\right)$ ). Consequently, by Theorem 3.3.45, the vertices of the Newton-Okounkov body $\Delta_{\nu}(D)$ are

$$
(0,0), Q_{14}=(14,0), Q_{17}=(15,3) \text { and } Q_{18}=(17,3)
$$

because $\theta_{2}^{r}(D)=a \nu_{r}\left(\varphi_{F_{1}}\right)-b\left(\nu_{r}\left(\varphi_{M_{1}}\right)-\delta \nu_{r}\left(\varphi_{F_{1}}\right)\right)=-1<0$. We depict the Newton-Okounkov body $\Delta_{\nu}(2 F+3 M)$ and the triangle $\mathfrak{C}(\nu) \cap \mathfrak{H}_{2 F+3 M}(\nu)$ in Figure 3.7 .

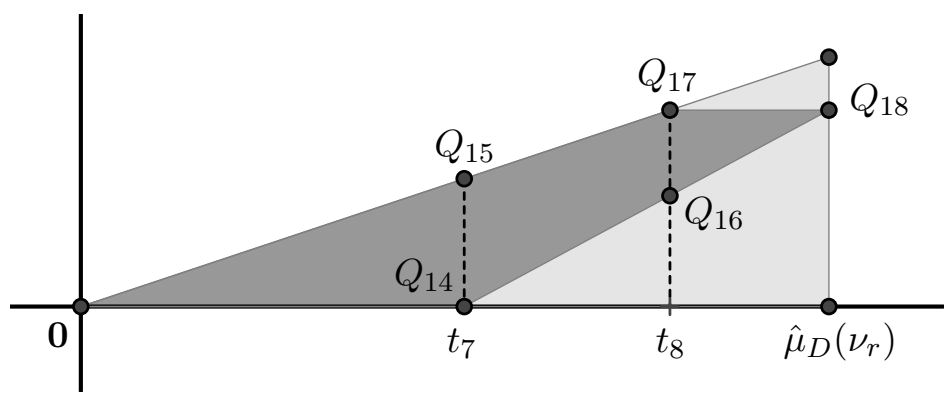

Figure 3.7: $\Delta_{\nu}(2 F+3 M)$ and $\mathfrak{C}(\nu) \cap \mathfrak{H}_{2 F+3 M}(\nu)$ in Example 3.3.46. 


\section{Conclusions}

Let $\mathbb{F}_{\delta}$ be a Hirzebruch surface, $\delta$ a non-negative integer, and $\nu_{r}$ a divisorial valuation of the function field of $\mathbb{F}_{\delta}$ centered at $\mathcal{O}_{\mathbb{F}_{\delta}, p}$, where $p$ is a point in $\mathbb{F}_{\delta}$ (we say for short that $\nu_{r}$ is a divisorial valuation of $\mathbb{F}_{\delta}$ ).

Due to the particular geometric structure of $\mathbb{F}_{\delta}$, in this thesis we distinguish two types of divisorial valuations of $\mathbb{F}_{\delta}$ : special and non-special valuations (see Definition 2.2.1). We introduce the concepts of non-positivity and negativity at infinity of a divisorial valuation of $\mathbb{F}_{\delta}$, concepts which we have adapted to special and non-special valuations by using adequate affine charts on $\mathbb{F}_{\delta}$. One of these charts is formed by the points in $\mathbb{F}_{\delta}$ which do not belong to the fiber $F_{1}$ containing $p$ nor the special section $M_{0}$; the second one is determined by the points which are neither in $F_{1}$ nor in a specific curve $M_{1} \neq M_{0}$.

Theorem 2.3.7 (respectively, Theorem 2.4.8) shows interesting global and local geometrical properties which are equivalent to the fact that a special (respectively, non-special) divisorial valuation $\nu_{r}$ of $\mathbb{F}_{\delta}$ is non-positive at infinity. In particular, the cone of curves $\operatorname{NE}(Z)$ of the rational surface $Z$ defined by a non-positive at infinity special (respectively, non-special) divisorial valuation $\nu_{r}$ is generated by the classes of the strict transforms of $F_{1}$ and $M_{0}$ (respectively, $F_{1}, M_{0}$ and $M_{1}$ ) and the classes of the strict transforms of the exceptional divisors associated with the composition of point blowups $\pi: Z \rightarrow \mathbb{F}_{\delta}$ given by $\nu_{r}$. This property also characterizes the above mentioned divisorial valuations.

Consider the flag $E_{\bullet}=\left\{Z \supset E_{r} \supset\{q\}\right\}$, where $E_{r}$ is the exceptional divisor defining a non-positive at infinity special, or non-special, divisorial valuation $\nu_{r}$ of $\mathbb{F}_{\delta}$ over $\mathbb{C}$ and $Z$ the rational surface given by $\nu_{r}$. In Theorem 3.2.1 we explicitly compute a Seshadri-type constant for pairs $\left(\nu_{r}, D\right)$, where $D$ is a big divisor on $\mathbb{F}_{\delta}$, denoted $\hat{\mu}_{D}\left(\nu_{r}\right)$. In addition, Theorems 3.3.7, 3.3.21, 3.3.24, 3.3.27, 3.3.42 and 3.3.45 determine the coordinates of the vertices of the Newton-Okounkov bodies of pairs $\left(E_{\bullet}, D\right)$. Our description considers two cases: $\nu_{r}$ is minimal with respect to $D$ and $\nu_{r}$ is non-minimal with respect to $D$ (see Definition 3.0.1).

The first components of the coordinates of the vertices of the Newton-Okounkov bodies of pairs $\left(E_{\bullet}, D\right)$, denoted $t_{i}$, are the values $t$ where the ray $[D]-t\left[E_{r}\right]$ crosses into a different Zariski chamber (see Subsection 1.5.2). Consequently, when $\nu_{r}$ is special (respectively, non-special), we obtain a local description of the cone of curves 
$\mathrm{NE}(Z)$ of $Z$ near the face $\left[\Lambda_{r}\right]^{\perp}$ (respectively, $\left[\Delta_{r}\right]^{\perp}$ ) generated by the classes of the strict transforms of $F_{1}, M_{0}$ (respectively, $F_{1}, M_{1}$ ) and the classes of the divisors $E_{1}, E_{2}, \ldots, E_{r-1}$, where $E_{1}, E_{2}, \ldots, E_{r-1}$ are the strict transforms of the exceptional divisors associated to $\pi$ (see Remark 3.3.18 and 3.3.39).

In [60] the authors consider divisorial valuations of $\mathbb{P}^{2}$ and study global and local geometry properties associated to non-positive and negative at infinity divisorial valuations $\nu$ of $\mathbb{P}^{2}$. In Section 2.3 we reprove [60, Theorems 1 and 2] as a particular case. The same happens with Corollary 3.3.29 where we obtain [65, Corollary 5.2] as a particular case. Notice that Corollary 5.2 in [65] computes the Newton-Okounkov bodies of pairs $\left(E_{\bullet}, D\right)$, where $D$ is a general projective line on $\mathbb{P}^{2}$ and $E_{\bullet}$ is a flag as $\{X \supset E \supset\{q\}\}, E$ being an exceptional divisor defining a non-positive at infinity divisorial valuation $\nu_{E}$ of $\mathbb{P}^{2}$ and $X$ the rational surface defined by $\nu_{E}$.

Finally, Section 2.5 studies and compares the dual graphs of non-positive at infinity divisorial and irrational valuations of $\mathbb{P}^{2}$ and $\mathbb{F}_{\delta}$ and provides an algorithm which inductively determines all dual graphs admitting non-positive at infinity valuations. 


\section{Conclusiones}

Sea $\mathbb{F}_{\delta}$ una superficie de Hirzebruch, $\delta$ un entero no negativo, y $\nu_{r}$ una valoración divisorial del cuerpo de funciones de $\mathbb{F}_{\delta}$ centrada en $\mathcal{O}_{\mathbb{F}_{\delta}, p}$, donde $p$ es un punto en $\mathbb{F}_{\delta}$ (abreviadamente, una valoración divisorial de $\mathbb{F}_{\delta}$ ).

En esta tesis, debido a la particular estructura geométrica de $\mathbb{F}_{\delta}$, distinguimos dos tipos de valoraciones divisoriales de $\mathbb{F}_{\delta}$, valoraciones especiales y no especiales (véase Definición 2.2.1). Introducimos el concepto de no positividad y negatividad en el infinito para valoraciones divisoriales de $\mathbb{F}_{\delta}$, conceptos adaptados a valoraciones especiales y no especiales. Para ello utilizamos cartas afines adecuadas en $\mathbb{F}_{\delta}$. Una de ellas está formada por los puntos de $\mathbb{F}_{\delta}$ que no pertenecen a la fibra $F_{1}$ que contiene a $p$, ni a la sección especial $M_{0}$. La segunda está determinada por los puntos que no están en $F_{1}$ ni en una curva particular $M_{1} \neq M_{0}$.

En el Teorema 2.3.7 (respectivemente, Teorema 2.4.8) mostramos interesantes propiedades geométricas globales y locales que son equivalentes al hecho de que una valoración especial (respectivamente, no especial) de $\mathbb{F}_{\delta}$ sea no positiva en el infinito. En particular, el cono de curvas $\mathrm{NE}(Z)$ de la superficie racional $Z$ definida por una valoración $\nu_{r}$ divisorial especial (respectivamente, no especial) no positiva en el infinito está generado por las clases de los transformados estrictos de $F_{1}$ y $M_{0}$ (respectivamente, $F_{1}, M_{0}$ y $M_{1}$ ) y las clases de los transformados estrictos de los divisores excepcionales asociados a la composición de explosiones $\pi: Z \rightarrow \mathbb{F}_{\delta}$ definidas por $\nu_{r}$. Esta propiedad también caracteriza las valoraciones divisoriales antes mencionadas.

Consideremos la bandera $E_{\bullet}=\left\{Z \supset E_{r} \supset\{q\}\right\}$, donde $E_{r}$ es un divisor excepcional que define una valoración divisorial $\nu_{r}$ especial, o no especial, de $\mathbb{F}_{\delta}$ (sobre $\mathbb{C})$ no positiva en el infinito y $Z$ la superficie racional dada por $\nu_{r}$. En el Teorema 3.2.1 calculamos explícitamente una constante de tipo Seshadri para pares $\left(\nu_{r}, D\right)$, donde $D$ es un divisor big en $\mathbb{F}_{\delta}$, denotada $\hat{\mu}_{D}\left(\nu_{r}\right)$. Además, en los Teoremas 3.3.7, 3.3.21, 3.3.24, 3.3.27, 3.3.42 and 3.3.45, expresamos las coordenadas de los vértices de los cuerpos de Newton-Okounkov de pares $\left(E_{\bullet}, D\right)$. Nuestra descripción considera dos casos: aquel donde $\nu_{r}$ es minimal respecto $D$ y el caso donde $\nu_{r}$ es no minimal respecto $D$ (véase Definición 3.0.1).

Las primeras componentes de las coordenadas de los vértices de los cuerpos de Newton-Okounkov de pares $\left(E_{\bullet}, D\right)$, denotadas $t_{i}$, son los valores $t$ donde el rayo 
$[D]-t\left[E_{r}\right]$ cruza a una cámara de Zariski diferente (véase Subsección 1.5.2). Consecuéntemente, cuando $\nu_{r}$ es especial (respectivamente, no especial), obtenemos una descripción local del cono de curvas $\mathrm{NE}(Z)$ de $Z$ cerca de la cara $\left[\Lambda_{r}\right]^{\perp}$ (respectivamente, $\left[\Delta_{r}\right]^{\perp}$ ) generado por las clases de los transformados estrictos de los divisores $F_{1}, M_{0}$ (respectivamente, $F_{1}, M_{1}$ ) y las clases de los divisores $E_{1}, E_{2}, \ldots, E_{r-1}$, donde $E_{1}, E_{2}, \ldots, E_{r-1}$ son los transformados estrictos de los divisores excepcionales asociados a $\pi$ (véase Notas 3.3.18 y 3.3.39).

En [60] los autores consideran valoraciones divisoriales de $\mathbb{P}^{2}$ y estudian propiedades geométricas globales y locales asociadas a valoraciones divisoriales de $\mathbb{P}^{2}$ no positivas y negativas en el infinito. En la Sección 2.3 probamos [60, Teoremas 1 y 2] como un caso particular. Lo mismo ocurre con el Corolario 3.3.29 donde obtenemos [65, Corolario 5.2] como un caso particular. Nótese que el Corolario 5.2 en [65] calcula explícitamente los cuerpos de Newton-Okounkov de pares $\left(E_{\bullet}, D\right)$, donde $D$ es una recta proyectiva general en $\mathbb{P}^{2}$ y $E_{\bullet}$ es una bandera como $\{X \supset E \supset\{q\}\}$, siendo $E$ un divisor excepcional que define una valoración divisorial $\nu_{E}$ de $\mathbb{P}^{2}$ (sobre $\mathbb{C}$ ) no positiva en el infinito y $X$ la superficie racional definida por $\nu_{E}$.

Finalmente, en la Sección 2.5 estudiamos y comparamos los grafos duales de valoraciones divisoriales e irracionales de $\mathbb{P}^{2}$ y $\mathbb{F}_{\delta}$ que son no positivas en el infinito y aportamos un algoritmo que determina inductivamente todos los grafos duales que admiten valoraciones no positivas en el infinito. 


\section{References}

[1] S. Abhyankar. Local uniformization on algebraic surfaces over ground fields of characteristic $p \neq 0$. Ann. Math. (2), 63:491-526, 1956. 2, 22

[2] S. Abhyankar. On the valuations centered in a local domain. Amer. J. Math., 78:321-348, 1956. 2, 22

[3] S. S. Abhyankar and T. T. Moh. Newton-Puiseux expansion and generalized Tschirnhausen transformation. I, II. J. Reine Angew. Math., 260:47-83; ibid. 261 (1973), 29-54, 1973. 3

[4] S. S. Abhyankar and T.T. Moh. On the semigroup of a meromorphic curve. I. In Proceedings of the International Symposium on Algebraic Geometry (Kyoto Univ., Kyoto, 1977), pages 249-414. Kinokuniya Book Store, Tokyo, 1978. 3

[5] M. Alberich-Carramiñana. Geometry of the Plane Cremona Maps, volume 179 of Lect. Notes in Math. Springer, 2002. 20

[6] M. S. Barnabé, J. Novacoski, and M. Spivakovsky. On the structure of the graded algebra associated to a valuation. J. Algebra, 560:667-679, 2020. 2

[7] T. Bauer, A. Küronya, and T. Szemberg. Zariski chambers, volumes, and stable base loci. J. Reine Angew. Math., 576:209-233, 2004. 4, 5, 44

[8] T. Bauer, S. Di Rocco, B. Harbourne, M. Kapustka, A. Knutsen, W. Syzdek, and T. Szemberg. A primer on Seshadri constants. In Interactions of classical and numerical algebraic geometry, volume 496 of Contemp. Math., pages 33-70. Amer. Math. Soc., Providence, RI, 2009. 40, 41

[9] A. Beauville. Complex Algebraic Surfaces, volume 34 of London Math. Soc. Student Texts. Cambridge University Press, Cambridge, second edition, 1996. $6,15,17,18,44,45,46,47,58$

[10] C. Birkar. Birational geometry of algebraic varieties. In Proc. Int. Cong. of Math. 2018. Vol. II., pages 583-608. 1

[11] C. Birkar and J. Waldron. Existence of Mori fibre spaces for 3-folds in char $p$. Adv. Math., 313:62-101, 2017. 1 
[12] H. Blum and M. Jonsson. Thresholds, valuations, and K-stability. Adv. Math., 365:107062, 2020. 4

[13] S. Boucksom. Corps d'Okounkov (d'après Okounkov, Lazarsfeld-Mustaţă et Kaveh-Khovanskii). Astérisque, (361):Exp. No. 1059, vii, 1-41, 2014. 4

[14] S. Boucksom, C. Favre, and M. Jonsson. Differentiability of volumes of divisors and a problem of Teissier. J. Alg. Geom., 18(2):279-308, 2009. 5

[15] S. Boucksom, A. Küronya, C. Maclean, and T. Szemberg. Vanishing sequences and Okounkov bodies. Math. Ann., 361:811-834, 2015. 4, 5, 6, 40, 42, 88, 94

[16] A. Campillo, F. Delgado, and S. M. Gusein-Zade. On the topological type of a set of plane valuations with symmetries. Math. Nachr., 290(13):1925-1938, 2017. 2

[17] A. Campillo and G. González-Sprinberg. On characteristic cones, clusters and chains of infinitely near points. In Singularities (Oberwolfach, 1996), volume 162 of Progr. Math., pages 251-261. Birkhäuser, Basel, 1998. 2

[18] A. Campillo, G. Gonzalez-Sprinberg, and M. Lejeune-Jalabert. Clusters of infinitely near points. Math. Ann., 306(1):169-194, 1996. 17, 20

[19] A. Campillo, G. Gonzalez-Sprinberg, and F. Monserrat. Configurations of infinitely near points. São Paulo J. Math. Sci., 3(1):115-160, 2009. 17

[20] A. Campillo, O. Piltant, and A. Reguera-López. Cones of curves and of line bundles on surfaces associated with curves having one place at infinity. Proc. London Math. Soc. (3), 84(3):559-580, 2002. 3

[21] C. Casagrande, G. Codogni, and A. Fanelli. The blow-up of $\mathbb{P}^{4}$ at 8 points and its Fano model, via vector bundles on a del Pezzo surface. Rev. Mat. Complut., 32(2):475-529, 2019. 1

[22] E. Casas-Alvero. Singularities of plane curves, volume 276 of London Math. Soc. Lect. Notes Ser. Cambridge Univ. Press, 2000. 17, 19, 21, 27

[23] P. Cascini, H. Tanaka, and C. Xu. On base point freeness in positive characteristic. Ann. Sci. Éc. Norm. Supér. (4), 48(5):1239-1272, 2015. 1

[24] A.-M. Castravet. Mori dream spaces and blow-ups. In Algebraic geometry: Salt Lake City 2015, volume 97 of Proc. Sympos. Pure Math., pages 143-167. Amer. Math. Soc., Providence, RI, 2018. 1

[25] S. Choi, S. Jung, J. Park, and J. Won. A product formula for volumes of divisors via Okounkov bodies. Int. Math. Res. Not. IMRN, (22):7118-7137, 2019. 5 
[26] S. R. Choi, Y. Hyun, J. Park, and J. Won. Asymptotic base loci via Okounkov bodies. Adv. Math., 323:784-810, 2018. 4

[27] S. R. Choi, Y. Hyun, J. Park, and J. Won. Okounkov bodies associated to pseudoeffective divisors. J. Lond. Math. Soc. (2), 97(2):170-195, 2018. 4

[28] C. Ciliberto, M. Farnik, A. Küronya, V. Lozovanu, J. Roé, and C. Shramov. Newton-Okounkov bodies sprouting on the valuative tree. Rend. Circ. Mat. Palermo (2), 66:161-194, 2017. 5, 6, 43

[29] I. Coskun and A. Prendergast-Smith. Fano manifolds of index $n-2$ and the cone conjecture. Math. Proc. Cambridge Philos. Soc., 166(1):1-31, 2019. 1

[30] D.A. Cox. The homogeneous coordinate ring of a toric variety. J. Alg. Geom., 4:17-50, 1995. 48

[31] S. D. Cutkosky, L. Ein, and R. Lazarsfeld. Positivity and complexity of ideal sheaves. Math. Ann., 321(2):213-234, 2001. 6, 41

[32] A. B. de Felipe and B. Teissier. Valuations and henselization. Math. Ann., 377:935-967, 2020. 2

[33] T. de Fernex. Negative curves on very general blow-ups of $\mathbb{P}^{2}$. In Projective varieties with unexpected properties, pages 199-207. Walter de Gruyter, Berlin, 2005. 2

[34] T. de Fernex. On the Mori cone of blow-up of the plane. 2010. arXiv:1001.5243. 2

[35] B. L. de la Rosa-Navarro, J. B. Frías-Medina, and M. Lahyane. Rational surfaces with finitely generated Cox rings and very high Picard numbers. $R A C$ SAM, 111:297-306, 2017. 2

[36] F. Delgado, C. Galindo, and A. Núñez. Saturation for valuations on twodimensional regular local rings. Math. Z., 234:519-550, 2000. 22, 27, 28, 29

[37] J. P. Demailly. Singular Hermitian metrics on positive line bundles. In Complex algebraic varieties (Bayreuth, 1990), volume 1507 of Lect. Notes Math., pages 87-104. Springer, Berlin, 1992. 5

[38] M. Dumnicki, B. Harbourne, A. Küronya, J. Roé, and T. Szemberg. Very general monomial valuations of $\mathbb{P}^{2}$ and a Nagata type conjecture. Comm. Anal. Geom., 25:125-161, 2017. 5, 6, 10, 40, 42, 88, 89, 90

[39] M. Dumnicki, A. Küronya, C. Maclean, and T. Szemberg. Rationality of Seshadri constants and the Segre-Harbourne-Gimigliano-Hirschowitz conjecture. Adv. Math., 303:1162-1170, 2016. 5 
[40] M. Dumnicki, A. Küronya, C. Maclean, and T. Szemberg. Seshadi constants via functions on Newton-Okounkov bodies. Math. Nachr., 289(17-18):2173-2177, 2016. 40,41

[41] L. Ein, R. Lazarsfeld, and M. Mustaţă. Contact loci in arc spaces. Compos. Math., 140(5):1229-1244, 2004. 2

[42] L. Ein, R. Lazarsfeld, M. Mustaţă, M. Nakamaye, and M. Popa. Asymptotic invariants of line bundles. Pure Appl. Math. Q., 1(2, Special Issue: In memory of Armand Borel. Part 1):379-403, 2005. 5

[43] L. Ein, R. Lazarsfeld, and K. Smith. Uniform approximation of Abhyankar valuations in smooth function fields. Amer. J. Math., 125:409-440, 2003. 42

[44] G. Ewald. Combinatorial Convexity and Algebraic Geometry, volume 168 of Graduate Texts in Mathematics. Springer-Verlag, New-York, 1996. 34

[45] A. Fahrner and J. Hausen. On intrinsic quadrics. Canad. J. Math., 72(1):145181, 2020. 1

[46] J. I. Farrán and C. Galindo. Evaluation codes and plane valuations. In Algebra for secure and reliable communication modeling, volume 642 of Contemp. Math., pages 119-157. Amer. Math. Soc., Providence, RI, 2015. 28

[47] C. Favre and M. Jonsson. The valuative tree, volume 1853 of Lect. Notes Math. Springer-Verlag, 2004. 3, 23, 27

[48] C. Favre and M. Jonsson. Eigenvaluations. Ann. Sci. Éc. Norm. Sup., 40:309349, 2007. 3

[49] C. Favre and M. Jonsson. Dynamical compactifications of $\mathbb{C}^{2}$. Ann. Math., 173:211-248, 2011. 3

[50] J.B. Frías-Medina and M. Lahyane. Harbourne-Hirschowitz surfaces whose anticanonical divisors consist only of three irreducible components. Int. J. Math., 29(12):1850072-1-1852272-19, 2018. 2

[51] J.B. Frías-Medina and M. Lahyane. The effective monoids of the blow-ups of Hirzebruch sufaces at points in general position. Rend. Circ. Mat. Palermo, (2). Ser. DOI:10.1007/s12215-020-00489-3, 2020. 2

[52] T. Fujita. On Zariski problem. Proc. Japan Acad. Ser. A Math. Sci., 55(3):106110, 1979. 43

[53] W. Fulton. Introduction to toric varieties, volume 131 of Annals of Mathematics Studies. Princeton Univ. Press, Princeton, NJ, 1993. 31, 33, 34 
[54] C. Galindo. Plane valuations and their completions. Comm. Algebra, 23(6):2107-2123, 1995. 26

[55] C. Galindo and F. Monserrat. On the cone of curves and of line bundles of a rational surface. Internat. J. Math., 15(4):393-407, 2004. 2

[56] C. Galindo and F. Monserrat. The cone of curves associated to a plane configuration. Comment. Math. Helv., 80(1):75-93, 2005. 2

[57] C. Galindo and F. Monserrat. $\delta$-sequences and evaluation codes defined by plane valuations at infinity. Proc. Lond. Math. Soc. (3), 98(3):714-740, 2009. 3,4

[58] C. Galindo and F. Monserrat. The Abhyankar-Moh Theorem for plane valuations at infinity. J. Algebra, 374:181-194, 2013. 4

[59] C. Galindo and F. Monserrat. Evaluation codes defined by finite families of plane valuations at infinity. Des. Codes Cryptogr., 70(1-2):189-213, 2014. 3, 4

[60] C. Galindo and F. Monserrat. The cone of curves and the Cox ring of rational surfaces given by divisorial valuations. Adv. Math., 290:1040-1061, 2016. ix, xi, 2, 3, 8, 54, 62, 134, 136

[61] C. Galindo, F. Monserrat, and C.-J. Moreno-Ávila. Discrete equivalence of non-positive at infinity plane valuations. Arxivmath:1911.06661, 2019. 7, 53

[62] C. Galindo, F. Monserrat, and C.-J. Moreno-Ávila. Seshadri-type constants and Newton-Okounkov bodies for non-positive at infinity valuations of Hirzebruch surfaces. Arxivmath:1905.03531, 2019. 7, 87

[63] C. Galindo, F. Monserrat, and C.-J. Moreno-Ávila. Non-positive and negative at infinity divisorial valuations of Hirzebruch surfaces. Rev. Mat. Complut., 33:349-372, 2020. 7, 53, 129

[64] C. Galindo, F. Monserrat, and J. Moyano-Fernández. Minimal plane valuations. J. Alg. Geom., 27:751-783, 2018. 5, 6, 10, 42, 87, 88, 89, 91

[65] C. Galindo, F. Monserrat, J. Moyano-Fernández, and M. Nickel. NewtonOkounkov bodies of exceptional curve valuations. Rev. Mat. Iberoam., 36(7):2147-2182, 2020. 5, 6, 10, 11, 26, 87, 90, 94, 95, 97, 99, 134, 136

[66] S. Greco and K. Kiyek. General elements of complete ideals and valuations centered at a two-dimensional regular local ring. In Algebra, arithmetic and geometry with applications (West Lafayette, IN, 2000), pages 381-455. Springer, Berlin, 2004. 3 
[67] K. Hanumanthu and A. Mukhopadhyay. Multi-point Seshadri constants on ruled surfaces. Proc. Amer. Math. Soc., 145(12):5145-5155, 2017. 5

[68] K. Hanumanthu and P. K. Roy. Seshadri constants on hyperelliptic surfaces. Proc. Amer. Math. Soc., 146(10):4175-4187, 2018. 5

[69] B. Harbourne. Blowings-up of $\mathbf{P}^{2}$ and their blowings-down. Duke Math. J., 52(1):129-148, 1985. 2

[70] B. Harbourne and M. Lahyane. Irreducibility of -1-classes on anticanonical rational surfaces and finite generation of the effective monoid. Pacific J. Math., 218(1):101-114, 2005. 2

[71] R. Hartshorne. Algebraic Geometry, volume 52 of Graduate Texts in Mathematics. Springer-Verlag, New York, 1977. 2, 13, 14, 15, 16, 17, 18, 31, 35, 36, $37,38,44,45,46,47$

[72] S. Iitaka. On D-dimensions of algebraic varieties. J. Math. Soc. Japan, 23:356373, 1971. 17, 67

[73] S. Iitaka. Algebraic geometry, volume 76 of Graduate Texts in Mathematics. Springer-Verlag, New York-Berlin, 1982. An introduction to birational geometry of algebraic varieties, North-Holland Mathematical Library, 24. 18

[74] K. Kaveh and A. Khovanskii. Newton-Okounkov bodies, semigroups of integral points, graded algebras and intersection theory. Ann. Math., 176:925-978, 2012. 4,94

[75] Y. Kawamata. The cone of curves of algebraic varieties. Ann. Math. (2), 119(3):603-633, 1984. 2

[76] S. Kleiman. Toward a numerical theory of ampleness. Ann. Math. (2), 84:293344, 1966. 1, 37

[77] J. Kollar and S. Mori. Birational Geometry of Algebraic Varieties, volume 134 of Cambridge Tracts Math. Cambridge University Press, Cambridge, 1998. 1, 17, 34, 36, 39

[78] A. Küronya and V. Lozovanu. Infinitesimal Newton-Okounkov bodies and jet separation. Duke Math. J., 166:1349-1376, 2017. 5

[79] A. Küronya and V. Lozovanu. Positivity of line bundles and Newton-Okounkov bodies. Doc. Math., 22:1285-1302, 2017. 4, 5

[80] A. Küronya and V. Lozovanu. Geometric aspects of Newton-Okounkov bodies. Banach Center Pub., 229:137-212, 2018. 4 
[81] A. Küronya and V. Lozovanu. Local positivity of linear series on surfaces. Alg. Numb. Th., 12(1):1-34, 2018. 4, 101

[82] A. Küronya, V. Lozovanu, and C. Maclean. Convex bodies appearing as Okounkov bodies of divisors. Adv. Math., 229:2622-2639, 2012. 4, 5, 11, 40, 43

[83] S. Lang and A. Néron. Rational points of abelian varieties over function fields. Amer. J. Math., 81:95-118, 1959. 35

[84] Serge Lang. Algebra, volume 211 of Graduate Texts in Mathematics. SpringerVerlag, New York, third edition, 2002. 38

[85] R. Lazarsfeld. Positivity in algebraic geometry I. Classical setting: line bundles and linear series, volume 48. Springer-Verlag, Berlin, 2004. 2, 4, 5, 31, 36, 37, 40, 41,43

[86] R. Lazarsfeld and M. Mustaţă. Convex bodies associated to linear series. Ann. Sci. Éc. Norm. Sup., 42:783-835, 2009. 4, 5, 11, 40, 43, 94

[87] Y. Liu and Z. Zhuang. Characterization of projective spaces by Seshadri constants. Math. Z., 289(1-2):25-38, 2018. 5

[88] K. Matsuki. Introduction to the Mori program. Universitext. Springer-Verlag, New York, 2002. 1

[89] R. Miranda and U. Persson. On extremal rational elliptic surfaces. Math. Z., 193(4):537-558, 1986. 2

[90] T. K. Moe. Cuspidal curves on Hirzebruch surfaces. PhD thesis, Department of Mathematics, University of Oslo, 2013. https://www.duo.uio.no/handle/ 10852/37197. 44, 48, 49

[91] F. Monserrat. El cono de curvas asociado a una superficie racional. Poliedricidad. PhD thesis, Departament de Matemàtiques, Universitat Jaume I, 2003. http://hdl.handle.net/10803/10499. 17, 19, 31, 34

[92] P. Montero. Newton-Okounkov bodies on projective bundles over curves. Math. Z., 291(3-4):1357-1379, 2019. 4

[93] S. Mori. Threefolds whose canonical bundles are not numerically effective. Ann. Math. (2), 116(1):133-176, 1982. 2

[94] B. Narasimha. On Mori cone of Bott towers. J. Algebra, 507:467-501, 2018. 1

[95] B. Narasimha. Erratum to "On Mori cone of Bott towers" [J. Algebra 507 (2018) 467-501]. J. Algebra, 527:447-450, 2019. 1 
[96] J. Novacoski and M. Spivakovsky. Reduction of local uniformization to the case of rank one valuations for rings with zero divisors. Michigan Math. J., 66(2):277-293, 2017. 2

[97] J. Novacoski and M. Spivakovsky. Key polynomials and pseudo-convergent sequences. J. Algebra, 495:199-219, 2018. 2

[98] D. Witt Nyström. Duality between the pseudoeffective and the movable cone on a projective manifold. J. Amer. Math. Soc., 32(3):675-689, 2019. With an appendix by Sébastien Boucksom. 5

[99] A. Okounkov. Brunn-Minkowski inequality for multiplicities. Invent. Math., 125:405-411, 1996. 4

[100] A. Okounkov. Why would multiplicities be log-concave? In The orbit method in geometry and physics (Marseille, 2000), volume 213 of Progr. Math., pages 329-347. Birkhäuser Boston, Boston, MA, 2003. 4

[101] J. Park and J. Shin. Seshadri constants and Okounkov bodies revisited. J. Pure Appl. Algebra, 225(2):106493, 2021. 4, 5

[102] M. Reid. Chapters on algebraic surfaces. In Complex algebraic geometry (Park city, UT, 1993), volume 3 of IAS/Park City Math. Ser., pages 3-159. Amer. Math. Soc., Providence, RI, 1997. 13, 44, 48

[103] R. T. Rockafellar. Convex Analysis. Princeton Mathematical Series, No. 28. Princeton University Press, Princeton, N.J., 1970. 31, 32, 33

[104] J. Roé. Local positivity in terms of Newton-Okounkov bodies. Adv. Math., 301:486-498, 2016. 4

[105] J. Roé and P. Supino. Nagata type statements. In Phenomenological approach to algebraic geometry, volume 116 of Banach Center Publ., pages 213-258. Polish Acad. Sci. Inst. Math., Warsaw, 2018. 2

[106] J. Rosoff. Effective divisor classes and blowing-up of $\mathbb{P}^{2}$. Pacific J. Math., 89(2):419-429, 1980. 2

[107] I. Shafarevich. Basic algebraic geometry. 1. Springer, Heidelberg, third edition, 2013. Varieties in projective space, Transleted from the 2007 Russian edition by Miles Reid. 13

[108] I. Shafarevich. Basic algebraic geometry. 2. Springer, Heidelberg, third edition, 2013. Schemes and complex manifolds, Translated from the 2007 third Russian edition by Miles Reid. 13 
[109] M. Spivakovsky. Valuations in function fields of surfaces. Amer. J. Math., 112:107-156, 1990. 2, 22, 23, 24, 27

[110] R. Svaldi. Hyperbolicity for log canonical pairs and the cone theorem. Selecta Math. (N.S.), 25(5):Paper No. 67, 23, 2019. 1

[111] B. Teissier. Valuations, deformations, and toric geometry. In Valuation theory and its applications, II (Saskatoon, SK, 1999), volume 33 of Fields Inst. Commun., pages 361-459. Amer. Math. Soc., Providence, RI. 2

[112] J. Xie. Intersections of valuation rings in $k[x, y]$. Proc. Lond. Math. Soc. (3), 111(1):240-274, 2015. 2

[113] X. Yuan and T. Zhang. Effective bounds of linear series on algebraic varieties and arithmetic varieties. J. Reine Angew. Math., 736:255-284, 2018. 5

[114] O. Zariski. The reduction of the singularities of an algebraic surface. Ann. Math. (2), 40:639-689, 1939. 2, 22

[115] O. Zariski. Local uniformization on algebraic varieties. Ann. Math. (2), 41:852896, 1940. 2, 22

[116] O. Zariski. The theorem of Riemann-Roch for high multiples of an effective divisor on an algebraic surface. Ann. Math., 76:560-615, 1962. 43

[117] O. Zariski and P. Samuel. Commutative Algebra II. Vol. II. Graduate Texts in Mathematics, Vol. 29, 1975. 2, 22

[118] Z. Zhuang. Fano varieties with large Seshadri constants. Adv. Math., 340:883913, 2018. 5 